\title{
SINGLE CHARGED PION PRODUCTION BY MUON NEUTRINOS IN THE MINERVA DETECTOR USING THE NUMI BEAM
}

\author{
by \\ Benjamin Messerly \\ B.A. in Physics, Bowdoin College, 2011 \\ M.S. in Physics, University of Pittsburgh, 2013
}

Submitted to the Graduate Faculty of

the Dietrich School of Arts and Sciences in partial fulfillment of the requirements for the degree of

Doctor of Philosophy

University of Pittsburgh

2019 


\section{UNIVERSITY OF PITTSBURGH \\ DIETRICH SCHOOL OF ARTS AND SCIENCES}

This dissertation was presented

by

Benjamin Messerly

It was defended on

December, 3rd 2019

and approved by

Vittorio Paolone, Professor of Physics

Rob Coalson, Professor of Chemistry

Steve Dytman, Professor Emeritus of Physics

Eric Swanson, Professor of Physics

Michael Wood-Vasey, Professor of Physics

Dissertation Director: Vittorio Paolone, Professor of Physics 
Copyright (C) by Benjamin Messerly

2019 


\title{
SINGLE CHARGED PION PRODUCTION BY MUON NEUTRINOS IN THE MINERVA DETECTOR USING THE NUMI BEAM
}

\author{
Benjamin Messerly, PhD \\ University of Pittsburgh, 2019
}

The next generation of long baseline neutrino oscillation experiments (DUNE, Hyper-K) aims to conclusively answer the outstanding questions in neutrino oscillation physics, including the nature of lepton CP-symmetry violation and the validity of the three-neutrino paradigm. The success of this program relies on excellent beam flux simulation and precisely-known cross sections for all neutrino-nucleus scattering processes. Currently, uncertainties on these models are large, and experiments such as MINERvA, located in the NuMI neutrino beamline at Fermilab, are dedicated to reducing them. This thesis makes improvements both to flux simulation models - via reduction in hadron focusing uncertainties in the G4NuMI simulation - and to cross section knowledge - via a pion production cross section measurement in the critical "transition region" between resonance and deep inelastic scattering. The cross section measurement performed is of muon neutrino charged current single charged pion production on hydrocarbon in the MINERvA detector and at mean neutrino energy of $6 \mathrm{GeV}$. The cross section is high-statistics, minimally-model dependent, and measured as a function of several muon, pion, and event-wide variables, including the first measurement of an invariant hadronic mass-like variable in the positive pion channel. Results are compared to the GENIE event generator, and found to broadly agree, though outstanding discrepancies remain in low four-momentum transfer squared and pion kinematics. 


\section{TABLE OF CONTENTS}

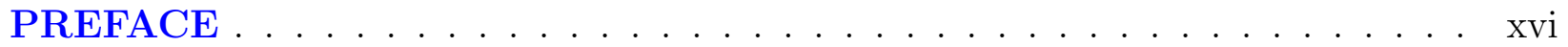

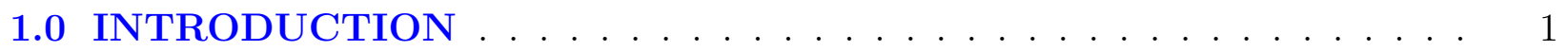

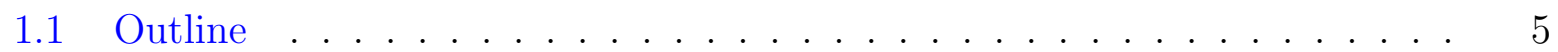

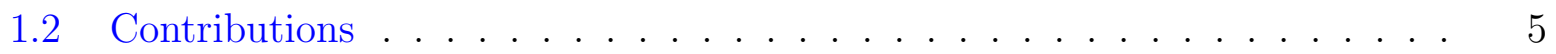

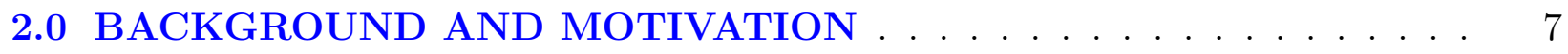

2.1 The Standard Model . . . . . . . . . . . . . . . . . . . . . . . . 7

2.1.1 The weak interaction . . . . . . . . . . . . . . . 9

2.1 .2 The strong interaction $\ldots \ldots \ldots \ldots$

2.1.3 Resonance particles . . . . . . . . . . . . . . . . . . . 11

2.2 Neutrino Oscillation . . . . . . . . . . . . . . . . . . . . . 12

2.2 .1 History of neutrino oscillation and mass . . . . . . . . . 13

2.2 .2 Neutrino oscillation formalism . . . . . . . . . . . . . . 13

2.2 .3 Neutrino oscillation parameters . . . . . . . . . . . 16

2.2.4 Long-baseline oscillation experiments . . . . . . . . . . . . . 19

2.2.5 The importance of flux and cross section knowledge to oscillation experiments . . . . . . . . . . . . . . . . 22

2.3 Charged current neutrino-nucleus interactions . . . . . . . . . . . . 30

2.3.1 Quasi-elastic and deep inelastic scattering . . . . . . . . . . 33

2.3.1.1 Quasi-elastic scattering formalism . . . . . . . . . . . 33

2.3.1.2 Deep inelastic scattering formalism . . . . . . . . . . . 34

2.3 .2 Resonance pion production $\ldots \ldots \ldots \ldots \ldots$

2.3 .3 Coherent pion production $\ldots \ldots \ldots \ldots \ldots$ 
2.3.4 Modifications to the nuclear medium . . . . . . . . . . . 37

2.3.4.1 Relativistic Fermi gas nuclear model . . . . . . . . . . . 39

2.3.4.2 Multi-nucleon correlations — random phase approximation (RPA) and Multi-nucleon npnh processes . . . . . . . 39

2.3.5 Nuclear final state interactions (FSI) . . . . . . . . . . . . 42

2.4 Previous measurements of $\nu_{\mu}$ CC single charged pion production . . . . . 43

3.0 NuMI NEUTRINO BEAM . . . . . . . . . . . . . . . . . . 45

3.1 Design . . . . . . . . . . . . . . . . . . . . 46

3.1 .1 Main Injector proton beam . . . . . . . . . . . . . . 46

$3.1 .2 \quad$ Target . . . . . . . . . . . . . . . . . 48

3.1 .3 Magnetic focusing horns . . . . . . . . . . . . . . 48

3.1.4 Decay pipe and absorbers . . . . . . . . . . . . . 51

3.2 Simulation and Flux Prediction . . . . . . . . . . . . . . . 52

4.0 MINERvA NEUTRINO DETECTOR . . . . . . . . . . . . 56

4.1 Design . . . . . . . . . . . . . . . . . 56

4.1 .1 Active tracking planes $\ldots \ldots \ldots \ldots \ldots$

4.1 .2 Subdetector regions . . . . . . . . . . . . . . . 60

4.1 .3 MINOS near detector . . . . . . . . . . . . . . . 60

4.1.4 Light detection, readout, and electronics . . . . . . . . . 62

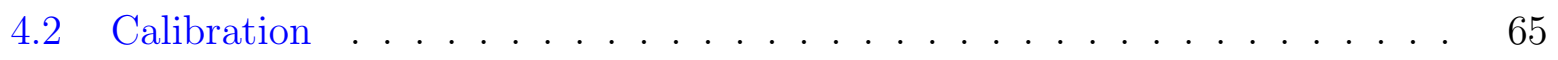

4.2 .1 ADC to $\mathrm{PE}$ calibration . . . . . . . . . . . . . 67

4.2.1.1 Pedestal subtraction and suppression . . . . . . . 67

4.2.1.2 FEB ADC-to-charge conversion . . . . . . . . . . 67

4.2.1.3 Photomultiplier tube gain . . . . . . . . . . . 68

4.2 .2 PE to energy calibration . . . . . . . . . . . . . . . . . 69

4.2.2.1 Optical fiber attenuation . . . . . . . . . . . . 70

4.2 .2 .2 Plane alignment . . . . . . . . . . . . . 71

4.2.2.3 Relative strip-to-strip response variations . . . . . . 71

4.2.2.4 Absolute detector energy scale (Muon equivalent unit) calibration ..................... 74 
4.2 .3 Time calibration . . . . . . . . . . . . . . . . 74

4.3 Data Reconstruction . . . . . . . . . . . . . . . . . . 77

4.3 .1 Time slicing . . . . . . . . . . . . . . . . . 77

4.3 .2 Clustering . . . . . . . . . . . . . . . . . . 78

4.3 .3 Tracking . . . . . . . . . . . . . . . . . . . 79

4.3 .4 Muon reconstruction . . . . . . . . . . . . . . . . . 82

4.3.5 Hadronic recoil energy . . . . . . . . . . . . . . . . . 83

4.4 Simulation . . . . . . . . . . . . . . . . . . 85

4.4 .1 Neutrino interaction simulation . . . . . . . . . . 85

4.4.2 GENIE neutrino interaction simulation . . . . . . . . . 86

4.4.2.1 GENIE neutrino interaction models . . . . . . . . . . 86

4.4.2.2 GENIE nuclear modifications and final state interactions . 87

4.4 .3 Detector simulation . . . . . . . . . . . . . . . 88

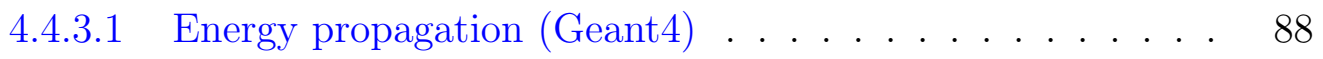

4.4.3.2 Data overlay . . . . . . . . . . . . . . . 88

4.4.3.3 Readout simulation . . . . . . . . . . . . . . 89

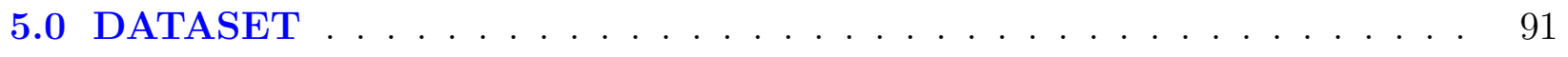

5.1 Dataset Description . . . . . . . . . . . . . . . . . . . . 91

5.2 Data Format, Organization, and Pipeline . . . . . . . . . . . . . 92

5.3 Simulated Monte Carlo data . . . . . . . . . . . . . . . . . . . 95

5.4 Event Weighting . . . . . . . . . . . . . . . . . . . . . 96

5.4 .1 Flux weights . . . . . . . . . . . . . . . . 96

5.4 .2 Muon tracking efficiency . . . . . . . . . . . . . . . 97

$5.4 .3 \quad$ MnvGENIE v1 . . . . . . . . . . . . . . . . . . . . . 97

\subsection{CHARGED PION DIFFERENTIAL CROSS SECTION MEASURE-}

MENT . . . . . . . . . . . . . . . . . . . . . . . . . . . . . 99

6.1 Overview of the measurement . . . . . . . . . . . . . . . . . 99

6.2 Signal definition . . . . . . . . . . . . . . . . . . . . . . . . . 101

6.2 .1 Analysis variable definitions . . . . . . . . . . . . . 103

6.3 Event selection . . . . . . . . . . . . . . . . . . . . 105 
6.3.1 Fiducial interaction vertex selection . . . . . . . . . . . . 107

6.3 .2 Muon selection . . . . . . . . . . . . . . . . . . . 107

6.3.3 Charged pion selection . . . . . . . . . . . . . . . . 108

6.3.3.1 Michel electron match . . . . . . . . . . . . . . . 108

6.3.3.2 Track particle identification (PID) score . . . . . . . . 111

6.3.3.3 Track terminal node energy . . . . . . . . . . . . 115

6.3 .4 Neutral pion veto . . . . . . . . . . . . . . . 116

6.3 .5 Kinematic cuts . . . . . . . . . . . . . . . 117

6.3.6 Alignment of signal definition, detector acceptance, and event selec-

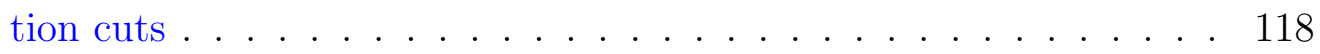

6.3.7 Event selection results . . . . . . . . . . . . . . . . . . . . . 119

6.4 Background subtraction $\ldots \ldots \ldots \ldots \ldots$

6.5 Unfolding . . . . . . . . . . . . . . . . . . . . . . . . 132

6.6 Efficiency correction and normalization . . . . . . . . . . . . 143

7.0 ERROR ANALYSIS . . . . . . . . . . . . . . . . . . . . . . . . 148

7.1 Many-universe uncertainty method . . . . . . . . . . . . . . . . 148

7.2 Model uncertainties . . . . . . . . . . . . . . . . . . . . 150

7.2 .1 Interaction model . . . . . . . . . . . . . . . . 150

7.2 .2 Final state interactions . . . . . . . . . . . . . . . . . 151

7.3 Detector uncertainties . . . . . . . . . . . . . . . . . . 154

$7.4 \quad$ Flux . . . . . . . . . . . . . . . . . . . 156

7.4.1 Hadron production uncertainties . . . . . . . . . 156

7.4 .2 Focusing uncertainties . . . . . . . . . . . . . . . 159

7.5 Cross section uncertainties . . . . . . . . . . . . . . . 161

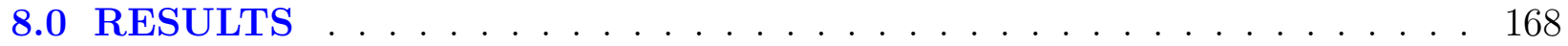

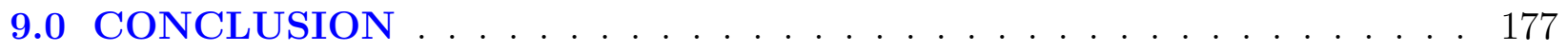

APPENDIX A. FLUX MODEL FOCUSING UNCERTAINTIES AND STUDY

OF FLUX SIMULATION DISCREPANCY . . . . . . . . . . . . . 178

A.1 Medium energy-era flux focusing uncertainties . . . . . . . . . . 178

A.1.1 General improvements to the flux model and calculation . . . . . 180 
A.1.2 Focusing parameter tolerance refinements . . . . . . . . 180

A.1.2.1 Proton beam spot size . . . . . . . . . . . 180

A.1.2.2 Proton beam baffle scraping . . . . . . . . . . . . 182

A.1.2.3 Target longitudinal position . . . . . . . . . . . . 183

A.1.2.4 Focusing horn currents . . . . . . . . . . . . . 183

A.1.2.5 Horn 1 geometry model . . . . . . . . . . . . . . 183

A.1.3 Focusing uncertainties by category . . . . . . . . . . . . 184

A.2 Data-Monte Carlo flux discrepancy in MINERvA . . . . . . . . . . 185

A.2.1 Neutrino interaction position heatmaps . . . . . . . . . 187

A.2.2 Neutrino mean interaction position . . . . . . . . . . . 189

A.2.3 MINERvA detector face "daisy" bin ratios . . . . . . . . . . . 189

A.2.4 Resolution to the discrepancy . . . . . . . . . . . . . 193

APPENDIX B. CROSS SECTION SYSTEMATIC UNCERTAINTY BREAK-

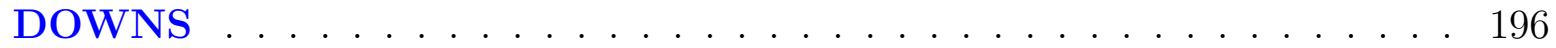

B.1 Detector uncertainties . . . . . . . . . . . . . . . 196

B.2 GENIE interaction model uncertainties . . . . . . . . . . . . . . . 201

B.3 GENIE FSI uncertainties . . . . . . . . . . . . . . . . . . 206

APPENDIX C. MINERvA MANY-UNIVERSE SYSTEMATICS SOFT-

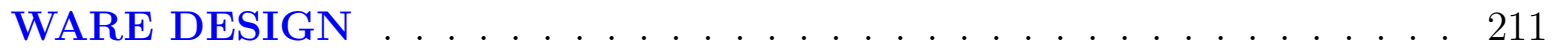

C.1 Basic systematics framework use . . . . . . . . . . . . . . . . . . 212

C.2 Universe classes . . . . . . . . . . . . . . . . . . . . . . 213

C.3 The HistWrapper class . . . . . . . . . . . . . . . . . . . . . . . 214

C.4 Template output and sample code . . . . . . . . . . . . . . . . 215

APPENDIX D. THE MINERvA COLLABORATION . . . . . . . . . . . 223

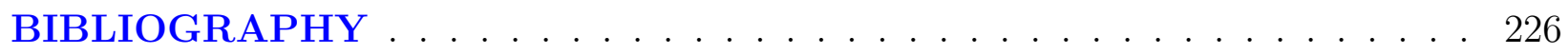




\section{LIST OF TABLES}

2.1 Summary of particles relevant to this thesis $\ldots \ldots \ldots 9$

2.2 Best-fit oscillation parameters assuming three-neutrino mixing $\ldots \ldots \ldots$

2.3 Summary of fundamental neutrino-nucleon interaction channels . . . . . . . 31

5.1 Information contained in root data files . . . . . . . . . . . . . . 96

6.1 Michel fit category and corresponding maximum separation cut value . . . . 110

6.2 Track terminal node energy cut values by node . . . . . . . . . . . . 115

6.3 Cross section normalization constants . . . . . . . . . . . . . . 147

7.1 GENIE interaction model uncertainty sources . . . . . . . . . . . . 151

7.2 GENIE FSI uncertainty sources . . . . . . . . . . . . . . . . 153

7.3 Particle energy fractional uncertainties . . . . . . . . . . . . . . . 154

7.4 Beamline focusing parameters . . . . . . . . . . . . . . 160 


\section{LIST OF FIGURES}

1.1 Mass of neutrino detectors through time . . . . . . . . . . . . . . 2

1.2 Long-baseline neutrino experiment DUNE schematic . . . . . . . . . . . . . 4

2.1 Period table of the standard model . . . . . . . . . . . . . . . . 8

2.2 Weak CC interation examples . . . . . . . . . . . . . . . 10

2.3 Neutrino-induced $\Delta^{+}$resonance pion production feynman diagram . . . . . . 12

2.4 Neutrino mass hierarchy . . . . . . . . . . . . . . . . . . . . 17

2.5 Long-baseline neutrino experiment DUNE schematic . . . . . . . . . . . 19

2.6 Neutrino fluxes for current and future oscillation experiments . . . . . . . . 20

2.7 Example far detector event rate and corresponding oscillation probability . . 21

2.8 DUNE sensitivity to oscillation parameters vs. systematic uncertainties . . . 23

2.9 T2K and NoVA systematic uncertainties. . . . . . . . . . . . . . . 24

2.10 NoVA near and far detector construction . . . . . . . . . . . . . . 26

2.11 Neutrino fluxes for current and future oscillation experiments . . . . . . . 27

2.12 NoVA procedure for extrapolating spectrum N to F. . . . . . . . . . . . 28

2.13 Initial and final state nuclear effects . . . . . . . . . . . . . . . . . . 29

2.14 Neutrino-nucleus charged current scattering interaction channel diagrams and total cross section . . . . . . . . . . . . . . . . . . 32

2.15 CC coherent pion production Feynman diagram . . . . . . . . . . . . 37

2.16 MiniBooNE CCQE $\nu_{\mu}$ cross section data and several predictions with alternate nuclear models . . . . . . . . . . . . . . . . . . . . 38

2.17 Depictions of $1 \mathrm{p} 1 \mathrm{~h}$ and $2 \mathrm{p} 2 \mathrm{~h}$ processes. . . . . . . . . . . . . . . . 40

2.18 MEC Feynman diagrams for electron scattering . . . . . . . . . . . . . 41 
3.1 Simulated fluxes for Low and Medium energy eras through MINERvA and

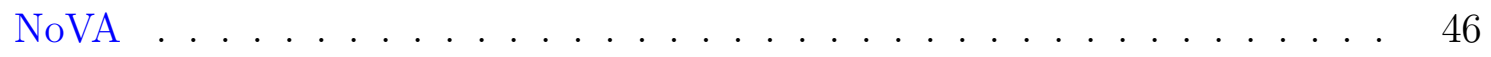

3.2 Overview of the Fermilab accelerator complex . . . . . . . . . . . 47

3.3 Schematic of the NuMI components . . . . . . . . . . . . . . . . 48

3.4 NuMI ME-era target . . . . . . . . . . . . . . . . . . . . . 49

3.5 NuMI horns . . . . . . . . . . . . . . . . . . . . . 49

3.6 Diagram of hadron focusing components . . . . . . . . . . . . . 50

3.7 ME NuMI flux by neutrino components . . . . . . . . . . . . . . 51

3.8 Pion phase space covered by NA49 data . . . . . . . . . . . . . . 54

3.9 Medium energy, hadron production constrained (ppfx) flux . . . . . . . 55

4.1 MINERvA detector side view and tracking plane front view . . . . . . . 58

$4.2 \mathrm{X}, \mathrm{U}$, and $\mathrm{V}$ plane orientations . . . . . . . . . . . . . . 59

4.3 Scintillator strip cross section and interleaved plane geometry . . . . . . . 59

4.4 MINERvA nuclear target region composition . . . . . . . . . . . . . 61

4.5 Muon track exiting MINERvA and entering MINOS . . . . . . . . . . . 62

4.6 MINOS near detector front view . . . . . . . . . . . . . . 62

4.7 MINERvA-MINOS-beam relative orientation diagram . . . . . . . . . . 63

4.8 Engineers drawing of a tracking plane connected with optical readout . . . . 64

4.9 Optical tube and PMT before construction . . . . . . . . . . . . 65

4.10 Schematic of a single optical readout channel . . . . . . . . . . . . . . 66

4.11 Example pedestal measurement for a single channel . . . . . . . . . . . 68

4.12 Front end board response constants . . . . . . . . . . . . . . . . 69

4.13 PMT operation diagram and typical gains distribution of all MINERvA channels 70

4.14 MINERvA plane alignment calibration . . . . . . . . . . . . . . 72

4.15 Fitted peak energy per unit path length for each plane . . . . . . . . . 73

4.16 Scintillator degredation shown through rock muon PE and the effect of the MEU calibration to correct the degredation . . . . . . . . . . . 75

4.17 Timing residual as a function of $\mathrm{PE}$ after calibrations . . . . . . . . . . 76

4.18 Time slices from a single readout gate . . . . . . . . . . . . . . 77

4.19 Event display showing a rock muon that is separated from a physics event . 78 


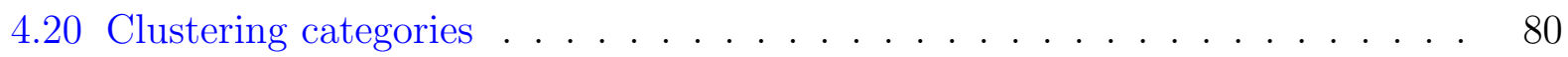

4.21 Illustration of long track reconstruction procedure . . . . . . . . . . . 81

4.22 Pion tracking efficiency as a funtion of momentum and angle . . . . . . . . 82

4.23 Fractional residual of $E_{\text {recoil }} \ldots \ldots \ldots \ldots$. . . . . . . . . . 84

5.1 Medium energy POT collected . . . . . . . . . . . . . . . . . . . . 92

5.2 Arachne event display showing a simulated $\mathrm{CC} 1 \pi^{+}$event $\ldots \ldots . . . . .994$

5.3 Arachne display of simulated hadron tracks in MINERvA . . . . . . . . . . 95

5.4 MINOS tracking efficiency correction . . . . . . . . . . . . . . . . 97

6.1 Hadronic energy fractional residual . . . . . . . . . . . . . . . . 105

6.2 Muon charge significance distribution . . . . . . . . . . . . . . . 108

6.3 Number of matched michels by signal and background . . . . . . . . . 111

6.4 Pion and proton Bethe curves with real and simulated data . . . . . . . . 113

6.5 Log-likelihood ratio particle identification score . . . . . . . . . . . . 114

6.6 The sum of the energy of the terminal and terminal +1 nodes for candidate pion tracks . . . . . . . . . . . . . . . . . 116

6.7 Isolated energy deposit distrubution (effective $\pi^{0}$ cut) $\ldots \ldots \ldots$. . . . 117

$6.8 W_{\text {exp }}$ distribution broken down by signal and background . . . . . . . 118

6.9 Event selection table by cut . . . . . . . . . . . . . . . . . 120

6.10 Event selection distributions for all analysis variables . . . . . . . . . . 124

6.11 Background sample as a function of $Q^{2} \ldots \ldots \ldots \ldots$

6.12 Signal and sideband regions, pre- and post-fit . . . . . . . . . . . 127

6.13 Background-subtracted distribution as a function of event variables . . . . 131

6.14 Migration matrices for all event variables . . . . . . . . . . . . 136

6.15 Unfolded distributions for all analysis variables . . . . . . . . . . . . 142

6.16 Efficiencies for all analysis variables . . . . . . . . . . . . . . . 147

7.1 Breakdown of the flux model uncertainty into its components: beam focusing and hadron production $(\mathrm{ppfx}) \ldots \ldots \ldots \ldots \ldots \ldots$

7.2 Breakdown of the hadron production flux uncertainty. . . . . . . . . . 158

7.3 Cross section systematic uncertainties . . . . . . . . . . . . 167 
8.1 MINERvA $\nu_{\mu} \mathrm{CC} \pi^{0}$ production cross section in $Q^{2}$ — in the low energy NuMI

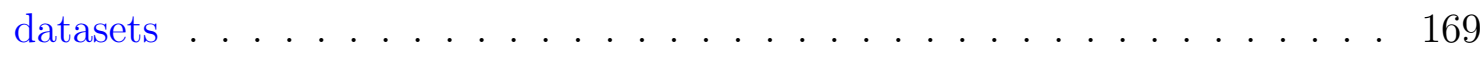

8.2 MINERvA pion production cross sections in $T_{\pi}$ - in the low energy and medium energy NuMI datasets . . . . . . . . . . . . . . . 170

8.3 Event selection in $T_{\pi}$, broken down by interaction channel and pion multiplicity 171

8.4 Cross section results for analysis variables . . . . . . . . . . . . 176

A.1 Low energy focusing uncertainties . . . . . . . . . . . . . . . . . 179

A.2 Medium energy focusing uncertainties . . . . . . . . . . . . . . 179

A.3 Measured proton beam spot size as a function as a function of intensity . . . 181

A.4 Proton beam spot size over three-year period of the ME run . . . . . . . . 182

A.5 Horn current over the ME run . . . . . . . . . . . . . . . . . . . . . . . . . . 184

A.6 ME focusing uncertainty breakdowns by category . . . . . . . . . . . . 185

A.7 MINERvA data-MC discrepancy in the low- $\nu$ selection . . . . . . . . . . 186

A.8 MINERvA detector face flux heat maps. . . . . . . . . . . . . . . 188

A.9 Mean neutrino x-y interaction position for shifted fluxes . . . . . . . . . . . 190

A.10 Daisy binning scheme on the front face of MINERvA . . . . . . . . . . . . . 191

A.11 Data-MC discrepancy broken down by daisy bins . . . . . . . . . . . . . . 191

A.12 Daisy bin flux ratios for a proton beam spot size and vertical horn 1 position

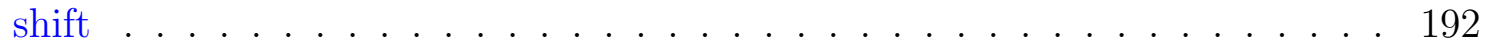

A.13 Daisy region flux ratios for target z-position shift . . . . . . . . . . . 193

A.14 $E_{\nu}$ ratio for shifts in the reconstructed muon energy compared to nominal

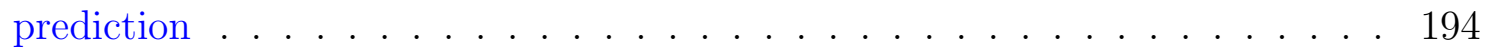

A.15 Ratios of low- $\nu$ sample data-to-MC and nominal-to- $1.75 \sigma$ shifted muon energy scale $\mathrm{MC} \ldots \ldots \ldots \ldots \ldots \ldots$

B.1 Cross section uncertainties due to detector effects . . . . . . . . . . . . 200

B.2 Cross section uncertainties due to GENIE interaction model effects . . . . . 205

B.3 Cross section uncertainties due to GENIE FSI effects . . . . . . . . . . . . . 210

C.1 Systematic universe objects inheritance structure . . . . . . . . . . . . 214

C.2 Depiction of HistWrapper and MnvH1D class structure . . . . . . . . . . . 215

C.3 Output of many-universe framework template code . . . . . . . . . . . 216 
D.1 MINERvA collaboration -2019 Pittsburgh, PA . . . . . . . . . . 223 


\section{PREFACE}

Thank you ...

To the MINERvA collaboration for accepting me and nurturing my physics career.

To all of the friends I've made at Fermilab and in the neutrino community.

To the University of Pittsburgh Department of Physics — professors, staff, and students

- for supporting me in my journey.

To my uncle Scott for your wisdom and counsel.

To Steve Dytman for countless insightful physics conversations.

To Vittorio Paolone for your willingness to throw me straight into the action, for your faith in me, and for your steady support.

To my parents. Despite no family history of scientists, you encouraged me on in this pursuit. Dad, for introducing me to the neutrino, and instilling in me a eagerness to understand the natural world. Mom, your enthusiasm for me as a person gives me unending encouragement.

To Lindsey. I would have enjoyed these last years a lot less without you. Thank you for you sacrifice and patience. You're the perfect copilot. 


\subsection{INTRODUCTION}

All of human experience - sights, sounds, smells, biology, chemistry — is made up of just a select few particles: photons make up the light we see, and electrons, protons, and neutrons make up all matter. ${ }^{1}$ But beyond your normal experience - in the burning sun and stars, in particle accelerators, and on unimaginably short timescales - there is a zoo of other particles that exist in the universe, and that do not constitute everyday matter. Three of these particles are neutrinos. Neutrinos are incredibly numerous in the universe, second only to photons. They are produced in massive quantities by the sun, and they are so weakly interacting that, despite the fact that 65 million neutrinos pass through every square centimeter of your body every second, night and day, it is likely that only one will ever collide with a proton, neutron, or electron in your body throughout your lifetime. In order to study neutrinos, massive, gymnasium-sized (and bigger) detectors are built underground or in antarctic ice. To reliably stop a neutrino would require a light-year of solid lead.

\footnotetext{
${ }^{1}$ To be sure, there are also some force mediating particles working behind the scenes to hold us together and give us mass. But even these only add a couple more particles to the short list that can explain all earthly phenomena.
} 


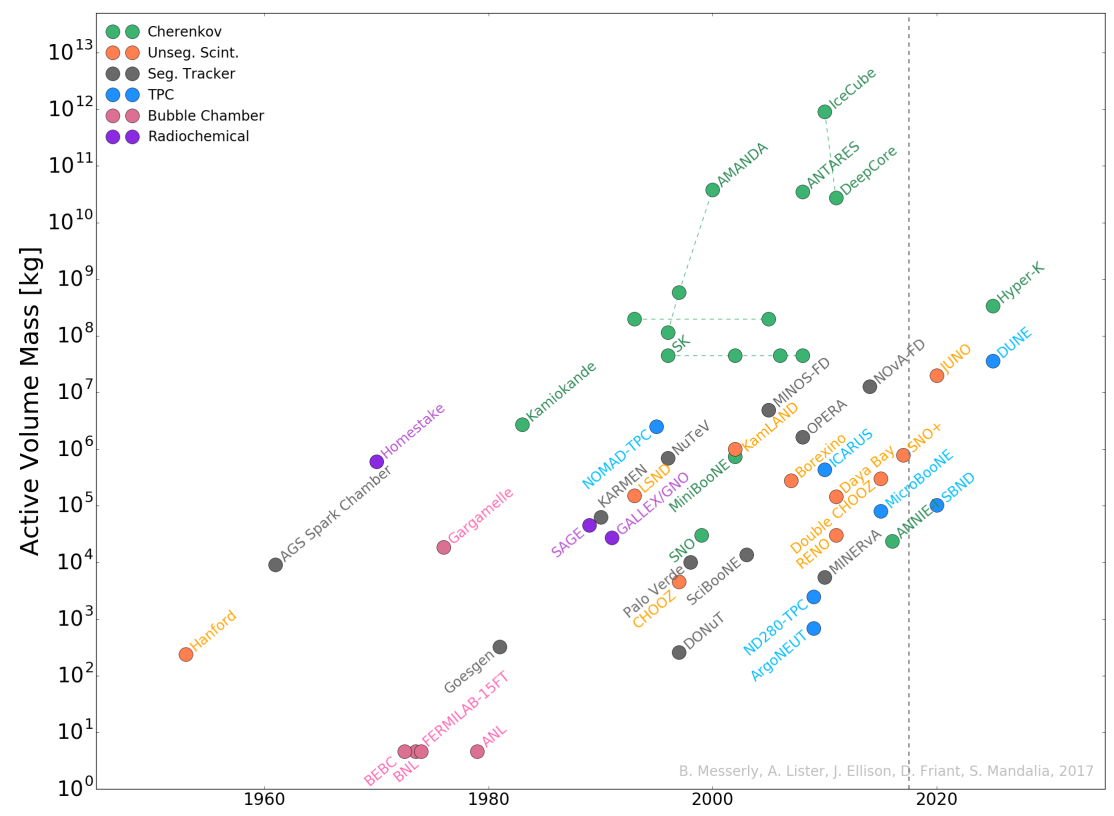

Figure 1.1: Mass of neutrino detectors through time. Instrumenting lakes and arctic ice have made incredibly massive neutrino detectors more affordable. Figure from [89].

The standard model of particles physics is the incredibly succesful theory that explains all of these particles and the interactions between them. It explains the everyday, earthly phenomena just as well as stellar, rare, short-lived, or high energy phenomena. But the standard model doesn't explain everything. The physics of black holes and general relativity, very large mass differences between particles, and the nature of dark matter and dark energy — these are all still mysteries not explained by the standard model.

That neutrinos oscillate between their three types was a fact not predicted by the original formulation of the standard model. The phenomena of oscillation means that a neutrino of electron type, which was created by some nuclear process, can travel some distance through space and be identified later as no longer an electron neutrino, but instead as one of the other neutrino types - muon or tau. Though not originally predicted, oscillation has been experimentally confirmed to occur.

Within the standard model, particles like neutrinos can oscillate only if they are massive 
(not massless, like the photon). It is in this way that neutrinos are believed to have mass. The implications are deep — through studying neutrino oscillations we may be able to learn about why matter and antimatter behave differently, or rather, why the universe has more matter than antimatter.

A worldwide endeavor to understand how neutrinos oscillate is currently underway. Intense muon neutrino beams from particle accelerators are directed through the earth, from a near detector to a far detector, and the neutrinos are counted at both locations - probabilistically, missing muon neutrinos oscillate away ("disappear") and electron (and sometimes tau) neutrinos "appear". The counting of neutrinos in order to measure oscillation probabilities turns out to be a serious challenge.

Knowing the number of neutrinos that pass through our detectors is hard. Not all neutrinos do interact in the detector. In fact the vast, vast majority do not interact. The number of neutrinos seen in the detector is the combination of the total number of neutrinos we start with (called the beam flux ) and the probability of a neutrino interacting (called the cross section). Ultimately, measuring the probability of oscillation uses detailed simulations of the neutrino beam flux and detectors, and it involves the application of complex standard model and nuclear physics as well as assumptions about the values of, sometimes yet poorlymeasured, neutrino cross sections. It is important to model and measure these well because at stake is the success of the neutrino oscillation physics program.

It gets worse: oscillation probabilities change depending on the energies of each neutrino, so each neutrino counted must be associated with a precise energy. Small errors in the energy determination of a neutrino can lead to incorrect probability measurements. And energy determination can be difficult. Neutrinos can interact with detectors in a variety of ways: they can interact with individual protons or neutrons, with the quarks inside of the nucleons, with pairs or groups of nucleons, and can scatter lightly or violently. In every case the signature of each neutrino interaction must be identified so its energy can be measured.

Furthermore, in order to increase the likelihood of neutrino interactions (as well as to make use of new detection technologies), detectors are built out of heavy nuclei, such as Carbon (6 protons and neutrons) or Argon (18 protons and neutrons). Unlike easier-tounderstand neutrino interactions with lighter nuclei, such materials complicate the picture, 
obscuring the interaction signature and the energy measurement. The ways that heavy nuclei obfuscate neutrino interactions are referred to as nuclear effects, initial state, and final state interactions.

For the upcoming neutrino oscillation experiment DUNE [40], which hopes to make conclusive measurements of many of the remaining oscillation parameters, the success of its program relies on excellent beam flux simulations and precisely-known cross sections for all neutrino interaction signatures and energies. Both beam flux mismodelings as well as large uncertainties or bad assumptions on neutrino interaction cross sections can bias oscillation measurements.

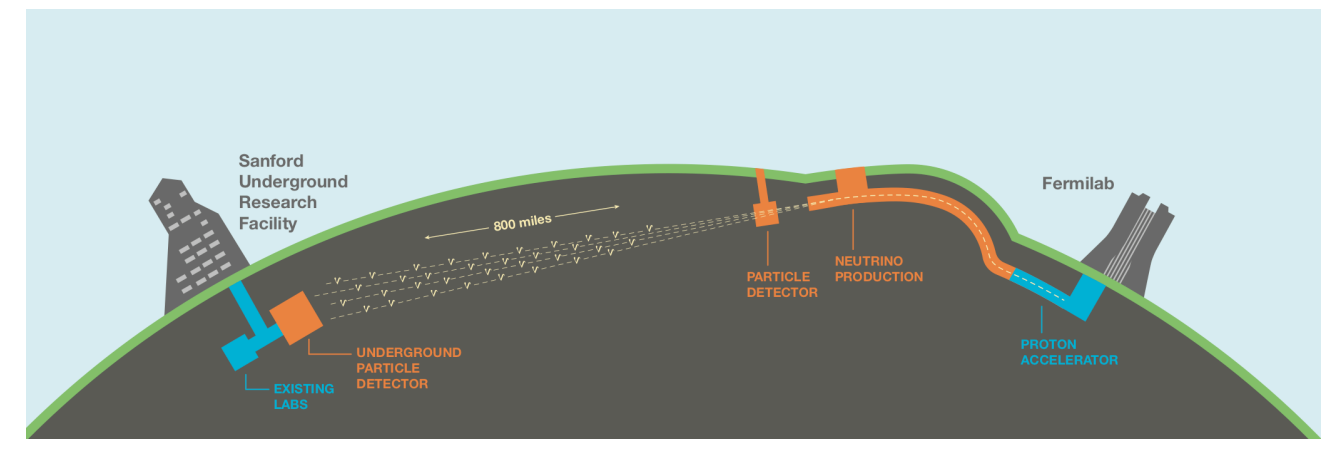

Figure 1.2: Experimental setup for the Deep Underground Neutrino Experiment (DUNE). Neutrinos will be sent $1300 \mathrm{~km}$ through the earth from Fermilab in Chicago to a detector in South Dakota.

This thesis makes concrete improvements to both flux simulation models and cross section knowledge that can be used by oscillation experiments, such as DUNE. In particular, regarding neutrino beam flux simulations, developments are made in the modeling of hadron focusing - more precisely evaluating focusing uncertainties and showing how beamline components can affect spatial distribution of neutrino spectra.

Regarding neutrino interaction signatures, this thesis measures the cross section for single charged pion production by muon neutrinos. Improved knowledge of this cross section is particularly important for DUNE's oscillation program, for it is a dominant interaction signature at the operating beam energy for DUNE, and it lies in a transition region between the better-understood lowest and highest energy regions. It is found that pion production 
cross sections models accurately measure the general features in data. However, DUNE's physics program demands very low cross section uncertanties, and precision modeling will be increasingly important. This thesis begins to probe models more deeply by measuring the pion production cross section in new event variables, with higher statistics, and with less model dependence.

\subsection{OUTLINE}

Chapter 2 provides context, motivation, and background physics for this measurement. Chapters 3 and 4 describe the experimental setup starting with the NuMI neutrino beam, and then the MINERvA neutrino detector, including its design, calibration, simulation, and data reconstruction. Chapter 5 discusses the dataset used for this measurement. Chapter 6 describes the analysis. Chapter 7 describes the error analysis, and Chapters 8 and 9 discuss results and conclude.

A secondary result of this thesis is the development in flux simulation knowledge. This work was performed in parallel to the cross section analysis, and because its methods and goals are so different from those of the cross section measurement, it has been placed in Appendix A.

\subsection{CONTRIBUTIONS}

This thesis refers to and cites figures from both published work and internal work performed by the MINERvA collaboration, the membership of which is listed in Appendix D.

Chapters 4 and 7 refer, in particular to [20]. Pion-specific reconstruction procedures were developed for the work in [45].

The contents of Chapter 6 were researched and created solely by the author, with exception to some subsections of 6.3 , which were studied together with one other MINERvA collaborator. 
The author maintained and improved several calibrations procedures described in Section 4.2.1, in particular the gains calibration of Section 4.2.1.3.

Appendix A contains the authors work related to the developments of the flux. Additional contributions were also made to the handling of the hadron production weighting of Section 3.2.

The author also designed and built a new software implementation of the many-universe systematic error calculation method. That work is described in Appendix C. 


\subsection{BACKGROUND AND MOTIVATION}

This chapter first introduces the standard model (2.1), which is the comprehensive theory underlying all of particle physics. Here, the fundamental forces and particles are introduced, along with resonance particles, which are specifically relevant to this measurement.

Next, neutrino oscillations are presented (2.2). Discussed here is the relationship of oscillation to neutrino mass, its mathematical formalism, its measurable parameters, the long-baseline experiments which study it, and, importantly, the relevance of flux and cross section knowlege (the topics of this thesis) to these experiments.

After oscillations, neutrino interactions are next discussed (2.3). More specifically it is charged current (CC) neutrino interactions on heavy nuclei, the type of interaction studied by this thesis measurement, that are investigated.

Finally, a literature review of previous $\nu_{\mu} \mathrm{CC}$ charged pion production measurements is presented (2.4).

\section{$2.1 \quad$ THE STANDARD MODEL}

The Standard Model (SM) is a quantum field theory that models 17 fundamental particles, as well as their antiparticle counterparts, and the forces that govern the interactions between them.

Of the 17 fundamental particles, 12 are fermions, defined by their half-integer intrinsic angular momentum, spin. The other five particles are bosons, defined by integer spin.

Among the bosons, the scalar Higgs $H$ boson generates particle mass, and the remaining four gauge bosons mediate three fundamental forces: electromagnetic $(\gamma)$, strong nuclear $(g)$, 
and weak nuclear $(W, Z)$.

The fermions can be separated into six leptons and six quarks. Among the leptons there are leptons of electric charge -1 (electron $e$, muon $\mu$, and tau $\tau$ ), each of which has a corresponding uncharged neutrino $\left(\nu_{e}, \nu_{\mu}, \nu_{\tau}\right)$. Three quarks have electric charge $+2 / 3$ (up $u$, strange $s$, top $t$ ) and the other three have electric charge $-1 / 3$ (down $d$, charm $c$, and bottom b). Quarks, however, are not observed in isolation, and only exist in bound states of two (mesons), three (baryons), or more rarely four or five, quarks. Further, all fermions can be separated into three generations, where the only difference between a fermion and its other-generation counterparts is mass.

Figure 2.1 maps the SM particles, and Table 2.1 summarizes the fundamental and composite particles most relevant to this thesis.

\section{Standard Model of Elementary Particles}

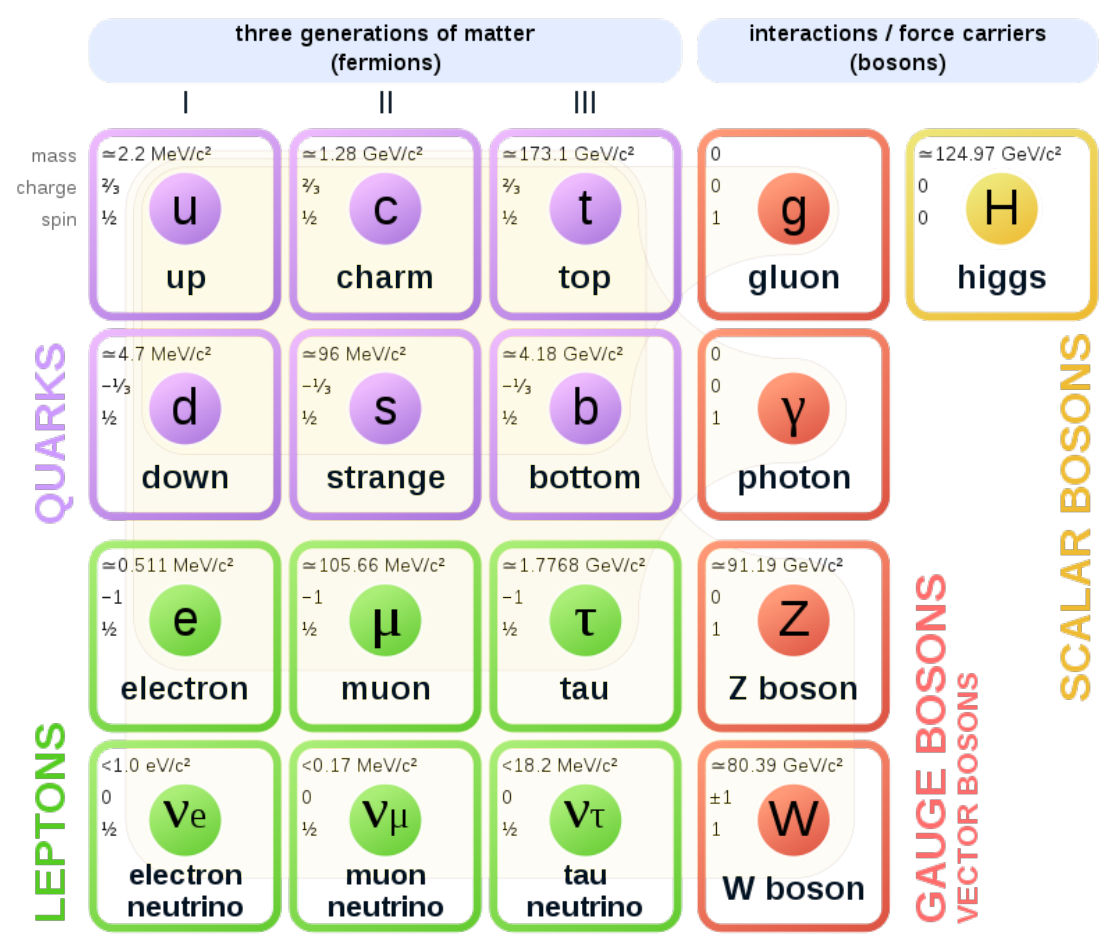

Figure 2.1: The "periodic table" of the standard model of particle physics. Figure from [93]. 
Table 2.1: Summary of fundamental and composite particles relevant to this thesis

\begin{tabular}{l|cccc} 
Paricle & Quark Content & Mass $\left(\mathrm{MeV} / c^{2}\right)$ & Lifetime & Decay Modes $(\%)$ \\
\hline $\mathrm{e}^{-}$ & & 0.5110 & $\infty$ & \\
\hline$\mu^{-}$ & & 105.7 & $2197.0 \mu \mathrm{s}$ & $\mathrm{e}^{-} \bar{\nu}_{e} \nu_{\mu}(100)$ \\
\hline $\mathrm{p}$ & $u u d$ & 938.2 & $\infty$ & \\
\hline $\mathrm{n}$ & $u d d$ & 939.6 & $880.2 \mathrm{~s}$ & $\mathrm{p} \mathrm{e}^{-} \bar{\nu}_{e}(100)$ \\
\hline$\pi^{+}$ & $u \bar{d}$ & 139.6 & $26.03 \mathrm{~ns}$ & $\mu^{+} \nu_{\mu}(99.99)$ \\
\hline$\Delta^{++}$ & $u u u$ & 1232. & $5.626 \mathrm{e}-24 \mathrm{~s}$ & $\mathrm{p} \pi^{+}(100)$ \\
\hline$\Delta^{+}$ & uud & 1232. & $5.626 \mathrm{e}-24 \mathrm{~s}$ & $\mathrm{p} \pi^{0}(66 \%)$ \\
& & & $\mathrm{n} \pi^{+}(33 \%)$ \\
\hline
\end{tabular}

\subsubsection{The weak interaction}

Both quarks and leptons are subject to weak interactions. Interactions mediated by the $W^{ \pm}$ are charged current (CC) and change the identity of the participating particle by 1 unit of electric charge. Among leptons, the charge current interactions takes place strictly within a generation, e.g. transforming a $\nu_{\mu}$ into a $\mu^{-}$. Examples of charged current interactions modifying leptons are shown in Figure 2.2.

The weak charged current in quarks is not so constrained to intragenerational conversions. Intergenerational mixing is possible because the quark weak eigenstates are different from their mass eigenstates. The Cabibbo-Kobayashi-Maskawa (CKM) matrix describes the couplings between the quark mass states.

The weak neutral current $(\mathrm{NC})$, mediated by the $Z^{0}$ does not change particle identies. In leptons, it enables interactions such as neutrino-electron scattering $\nu_{\mu}+\mathrm{e}^{-} \rightarrow \nu_{\mu}+\mathrm{e}^{-}$.

\subsubsection{The strong interaction}

The strong interaction, which only affects quarks, is mediated by gluons. Anologous to the electromagnetic charge, there is also a strong charge, called color charge. Like the EM charge, color charge is conserved at interaction vertices, but unlike the EM charge, color charge comes in three types (red, green, and blue), and it is carried both by quarks and the force mediator gluons. 


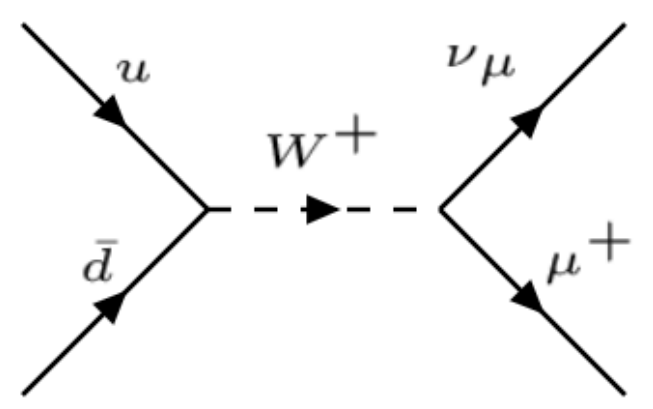

(a) Pion decay

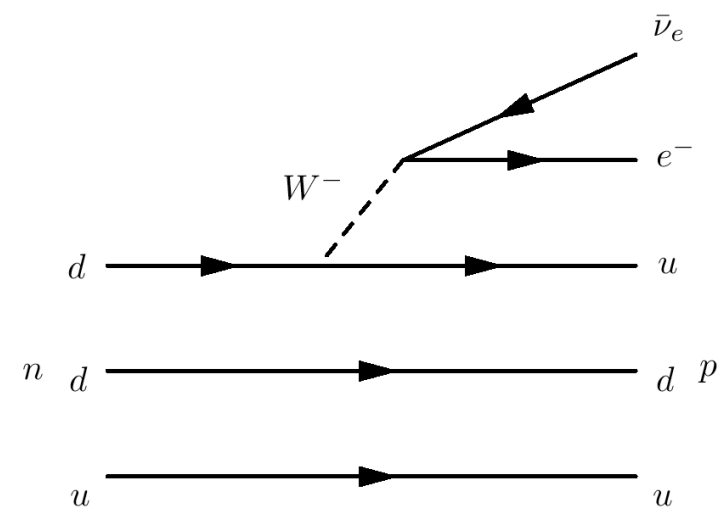

(b) Neutron decay

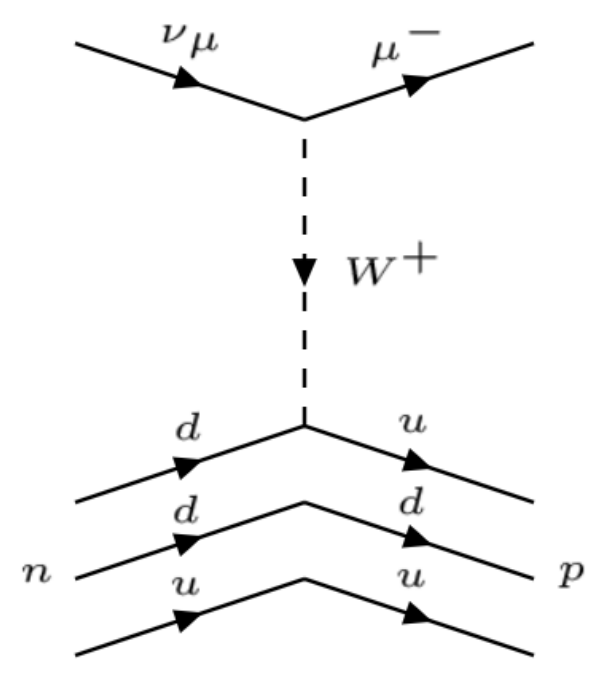

(c) Neutrino quasi-elastic scattering

Figure 2.2: Examples of weak charged-current interactions.

Bound quark states are called hadrons. Hadrons most commonly come in groups of two quarks - a meson $(q-\bar{q}$ pair $)$ - and three quarks - a baryon ( $q-q-q$ trio). In a phenomenon called color confinement, individual quarks are not observed in nature. Instead only colorless particles - mesons made of a color-anticolor pair or baryons made of a red-green-blue trio - 
are seen. When enough energy is supplied to a hadron, it undergoes hadronization wherein $q \bar{q}$ pairs are created from the vacuum to form new hadrons. Color confinement is not implied by the theory of strong interactions, but occurs as a consequence of gluons carrying color

charge. At a certain point it becomes energetically favorable to create new quarks from the vacuum than to continue separating the original quarks.

\subsubsection{Resonance particles}

Resonance particles are typically very short-lived particles for which direct detection is not possible, and whose existence is inferred from scattering experiments. Peaks in scattering cross sections occur at the resonance particle mass, and resonance decay products can be observed.

The $\Delta^{+}$resonance baryon for example can be created when a neutrino interacts with a neutron via the charged current (shown in Figure 2.3), transforming a down quark into an up quark at a weak interaction vertex. The lifetime of $\Delta$ baryons is $O\left(10^{-24}\right)$, so it decays quickly by emitting a gluon, which hadronizes forming a proton-pion set of product particles. At neutrino energies of a few-GeV, this is the dominant process by which single pions are produced.

Note that the quark content of the $\Delta^{+}$and the proton is the same. The $\Delta^{+}$differs in that it has spin and isospin of $3 / 2$, while the proton has spin and isospin of $1 / 2$. The $\Delta^{++}$, created from a neutrino scattering off of a proton, has three spin $+1 / 2 u$ quarks, but is exempt from the Pauli exclusion principle by virtue of the color charge.

Resonance production scattering cross sections are proportional to Breit-Wigner factor

$$
\sigma_{\mathrm{res}} \sim \frac{\Gamma}{\left(W-M_{\mathrm{res}}\right)^{2}+\Gamma^{2} / 4}
$$

which mathematically resembles the description of a harmonic oscillator being driven at its harmonic frequency. In this case, $\Gamma$ is the resonance width, $M_{\text {res }}$ is the mass of the resonance particle, and $W$ is the system's invariant mass. The width $\Gamma$ determines the range of $W$ over which the resonance particle can be produced. Through another point of view, the target hadron has a resonant frequency at $W=M_{\text {res }}$ around which it is in an excited state. 


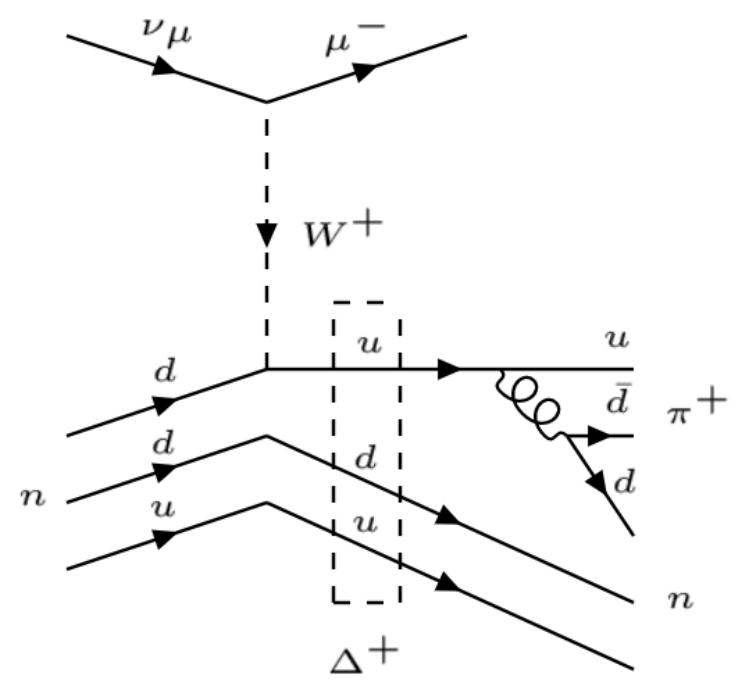

Figure 2.3: Feynman diagram showing neutrino-induced $\Delta^{+}$resonance pion production. The $\Delta^{+}$can also decay into a $\mathrm{p} \pi^{0}$ final state. The analogous scattering off of a proton produces a $\pi^{+}$and a $\mathrm{p}$.

\subsection{NEUTRINO OSCILLATION}

Neutrino oscillation is the phenomenon in which a neutrino of a certain flavor state (i.e. $\nu_{e}$, $\nu_{\mu}$, or $\nu_{\tau}$ ) has a probability of, after traveling some distance, being observed in a different flavor state. This section first discusses briefly why the neutrino was originally thought to be massless, how it came to be known to have mass, and the relationship between mass and oscillations. Next the mathematical formalism for oscillation is derived in the plane wave approximation, and its parameterization is discussed. Finally, the current state of neutrino oscillation research, via long baseline neutrino experiments, is discussed, along with the important role that neutrino beam flux and neutrino-nucleus interaction cross section knowledge plays in the oscillation physics program. 


\subsubsection{History of neutrino oscillation and mass}

In the earliest formulations of the SM, neutrinos were massless. The motivation for this choice was somewhat historical. In 1956, Chien-Shiung Wu measured the decay of ${ }^{60} \mathrm{Co}$ and determined that the weak interaction violated parity (the symmetry of spatial reflections), and maximally-so. The experiment only observed right-handed anti-neutrinos in the ${ }^{60} \mathrm{Co}_{27} \rightarrow{ }^{60} \mathrm{Ni}_{28}+\mathrm{e}^{-}+\bar{\nu}_{e}$ decay and no left-handed antineutrinos. From this result, TsungDao Lee and Chen-Ning Yang, the theorists who originally proposed the experiment, posited that neutrinos have inherent helicity, must travel at the speed of light, and therefore must be massless. This latter conclusion was not contradicted by experimental evidence at the time, and in 1958 it was further established as a feature of the $V$ - $A$ theory, a progenitor of the weak interaction in modern SM formalism. ${ }^{1}$

The first hints of neutrino oscillation were seen in the 1950's, and quickly a mechanism, which involved giving neutrinos mass, was developed by Bruno Pontecorvo, Ziro Maki, Masami Nakagawa, and Shoichi Sakata (PMNS) to explain the phenomenon.

Over the next decades, the canonical "textbook" SM formalism would mature assuming massless neutrinos and no oscillation. Meanwhile experimental evidence for neutrino oscillation mounted, and by the time it culminated in the 2015 Nobel Prize, a minimal and consistent neutrino oscillation extension to the SM formalism - one that gave neutrinos mass, was built on the original PMNS oscillation mechanism, and mirrored the well-established process that quarks undergo — was ready and waiting. This "new" standard model is working its way into textbooks as we speak.

\subsubsection{Neutrino oscillation formalism}

For the massive neutrino, the weak and mass eigenstates are identical, unlike for the massless neutrino, which could be created, propagate, and be detected in a definite state. Instead, massive neutrinos interact in states of definite flavor but propagate as states of definite mass.

\footnotetext{
${ }^{1}$ The V-A theory was attractive at the time, for in it the weak force violated parity maximally, in agreement with experiment, only interacting with left-handed components of neutrinos and right-handed components of antineutrinos. And thus, since neutrinos only interact via the weak force, the right handed neutrino and the left-handed antineutrino were predicted to never be observed.
} 
There is a mixing between a weak eigenstate $\alpha=e, \mu, \tau$ and mass states $i=1,2,3$ which can be written:

$$
\left|\nu_{\alpha}\right\rangle=\sum_{j} U_{\alpha j}\left|\nu_{j}\right\rangle
$$

where the matrix $U_{\alpha j}$ is the unitary $3 \times 3$ PMNS mixing or rotation matrix, named for its aforementioned authors.

Neutrino oscillations can then be derived by considering the propagation of a $\nu_{\alpha}$ through time. Starting from the usual plane wave expression for free propagation of the mass eigenstate $j$ :

$$
\left|\nu_{j}(t)\right\rangle=e^{-i\left(p_{j} \cdot x_{j}\right)}\left|\nu_{j}(0)\right\rangle
$$

with $p_{j}$ and $x_{j}$ the momentum and position four-vectors such that $p_{j} \cdot x_{j}=E_{j} t-\vec{p}_{j} \cdot \vec{x}$. The energy of component $j$ is given by the dispersion relation:

$$
E_{j}=\sqrt{\vec{p}_{j}^{2}+m_{j}^{2}}
$$

Next, we can assume that all massive neutrino components are aligned along the same direction and that $t \sim z$ for ultrarelativistic neutrinos. ${ }^{2}$ From these assumptions, the phase becomes:

$$
\begin{aligned}
-i\left(E_{j} t-\vec{p}_{j} \cdot \vec{x}\right) & \approx-i\left(E_{j}-p_{j}\right) z \\
& =-i \frac{E_{j}^{2}-p_{j}^{2}}{E_{j}+p_{j}} z \\
& =-i \frac{m_{j}^{2}}{2 E} z
\end{aligned}
$$

where $E$ ignores mass energy, and is thus the same for all components. Thus, for a neutrino that was produced in flavor state $\alpha$ and freely propagates distance $L$, the state is written:

$$
\left|\nu_{\alpha}(L)\right\rangle=\sum_{\beta}\left(\sum_{j} U_{\alpha j} U_{\beta j}^{*} e^{-i\left(m_{j}^{2} / 2 E\right) L}\right)\left|\nu_{\beta}\right\rangle
$$

\footnotetext{
${ }^{2} \mathrm{~A}$ more-appropriate wave packet treatment does not require these assumptions. Nevertheless, modifications to account for the assumptions can be shown to be negligible. See [57] for a comparison of the plane wave vs. wave packet treatments, as well as for an evaluation of the assumptions used here.
} 
where the unitarity of $U$ has been used to invert Equation 2.2. The probability that a neutrino created in flavor state $\alpha$ travels distance $L$ and is observed in flavor state $\beta$ (which may or may not be the same as $\alpha$ ) is:

$$
\begin{aligned}
P_{\nu_{\alpha} \rightarrow \nu_{\beta}}(L) & =\left|\left\langle\nu_{\beta} \mid \nu_{\alpha}(L)\right\rangle\right|^{2} \\
& =\left(\sum_{j} U_{\alpha j} U_{\beta j}^{*} e^{-i\left(m_{j}^{2} / 2 E\right) L}\right)^{*}\left(\sum_{k} U_{\alpha k} U_{\beta k}^{*} e^{-i\left(m_{k}^{2} / 2 E\right) L}\right) \\
& =\sum_{j} \sum_{k} U_{\alpha j}^{*} U_{\beta j} U_{\alpha k} U_{\beta k}^{*} e^{-i\left(m_{k}^{2}-m_{j}^{2}\right) L / 2 E} \\
& =\sum_{j} \sum_{k} U_{\alpha j}^{*} U_{\beta j} U_{\alpha k} U_{\beta k}^{*} e^{-i\left(\Delta m_{k j}^{2} L / 2 E\right)}
\end{aligned}
$$

where $\Delta m_{k j}^{2} \equiv m_{k}^{2}-m_{j}^{2}$. Equation 2.7 can be further simplified:

$$
\begin{aligned}
P_{\nu_{\alpha} \rightarrow \nu_{\beta}}(L)= & \sum_{j} \sum_{k} U_{\alpha j}^{*} U_{\beta j} U_{\alpha k} U_{\beta k}^{*}\left[1-2 \sin ^{2}\left(\frac{\Delta m_{k j}^{2} L}{4 E}\right)-i \sin \left(\frac{\Delta m_{k j}^{2} L}{2 E}\right)\right] \\
= & \sum_{j} \sum_{k} U_{\alpha j}^{*} U_{\beta j} U_{\alpha k} U_{\beta k}^{*} \\
& +\sum_{j \neq k} U_{\alpha j}^{*} U_{\beta j} U_{\alpha k} U_{\beta k}^{*}\left[-2 \sin ^{2}\left(\frac{\Delta m_{k j}^{2} L}{4 E}\right)-i \sin \left(\frac{\Delta m_{k j}^{2} L}{2 E}\right)\right]
\end{aligned}
$$

The first term is:

$$
\begin{aligned}
\sum_{j} \sum_{k} U_{\alpha j}^{*} U_{\beta j} U_{\alpha k} U_{\beta k}^{*} & =\sum_{j}\left|U_{\alpha j}^{*} U_{\beta j}\right|^{2} \\
& =\delta_{\alpha \beta}
\end{aligned}
$$

while the second and third terms can be simplified by using the symmetries of the sines and that $U_{j k}+U_{j k}^{*}=2 \Re\left(U_{j k}\right)$ and $U_{j k}-U_{j k}^{*}=-2 i \Im\left(U_{j k}\right)$. Thus the familiar probability expression is obtained:

$$
\begin{aligned}
P_{\nu_{\alpha} \rightarrow \nu_{\beta}}(L)= & \delta_{\alpha \beta}-4 \sum_{j>k} \Re\left(U_{\alpha j}^{*} U_{\beta j} U_{\alpha k} U_{\beta k}^{*}\right) \sin ^{2}\left(1.27 \frac{\Delta m_{j k}^{2} L}{4 E}\right) \\
& +2 \sum_{j>k} \Im\left(U_{\alpha j}^{*} U_{\beta j} U_{\alpha k} U_{\beta k}^{*}\right) \sin \left(2.54 \frac{\Delta m_{j k}^{2} L}{2 E}\right)
\end{aligned}
$$

The factor of 1.27 comes from returning the missing $c^{3} / \hbar$ and from expressing mass $\Delta m$ in $\mathrm{eV} / c$, length $L$ in $\mathrm{km}$, and energy $E$ in $\mathrm{GeV}$. 


\subsubsection{Neutrino oscillation parameters}

Observe in Equation 2.10 the frequency of oscillation is dependent on experimental parameters: $L$ the length between production and detection and $E$ the neutrino energy. These paramters must be adjusted for sensitivity to $\Delta m^{2}$.

Note that measurement of oscillations is not sensitive to the component absolute masses, but only to the mass splittings, $\Delta m_{k j}^{2}$. Absolute neutrino masses cannot be measured through oscillation. Also observe that because $\Delta m_{12}^{2}+\Delta m_{31}^{2}+\Delta m_{23}^{2}=0$, there are only two independent mass splittings.

Experiments have measured the mass splittings and found that two are very close in value, while the third is much larger. Using the established mass numbering convention, they are:

$$
\Delta m_{21}^{2} \ll\left|\Delta m_{31}^{2}\right| \sim\left|\Delta m_{32}^{2}\right|
$$

Because they appear as an argument to $\sin ^{2}$ in the oscillation probability expression, the signs of the mass splittings are generally not known. The sign of $\Delta m_{21}^{2}$ however has been determined through oscillation effects in matter: unlike in vacuum, oscillation through matter either enhances or suppresses electron neutrino appearance relative to electron antineutrino appearance, depending on the sign of $\Delta m_{21}^{2}$.

The sign of $\Delta m_{32}^{2}$ (and thus of $\Delta m_{31}^{2}$ ) is still unknown. This problem, whether the masses are ordered $m_{1}<m_{2}<m_{3}$ or $m_{3}<m_{1}<m_{2}$, is known as the neutrino mass hierarchy problem (depicted in Figure 2.4). The former configuration is referred to as the normal hierarchy, while the latter is the inverted hierarchy. The mass hierarchy is currently a research area of great interest, and it is one of the problems new long baseling experiments hope to be able to answer.

The value of $\Delta m_{21}^{2}$ is $\sim 7.4 \mathrm{e}-5 \mathrm{eV}^{2}$ and $\left|\Delta m_{31}^{2}\right|\left(\left|\Delta m_{32}^{2}\right|\right)$ is $\sim 2.5 \mathrm{e}-3 \mathrm{eV}^{2}$. With such a large separation between these splittings, their contributions to the oscillation probability equation (2.10) depend strongly on the experimental $L / E$ scale.

At $\Delta m_{21}^{2} L / E$ "small" relative to $\Delta m_{31}^{2} L / E \sim \pi$, the $\sin ^{2} \Delta m_{21}^{2}$ will not participate in the oscillation. $\Delta m_{32}^{2}$ is known as the atmospheric mass splitting, named for its relevance to the natural oscillation experiment in which neutrinos are created from cosmic ray activity 


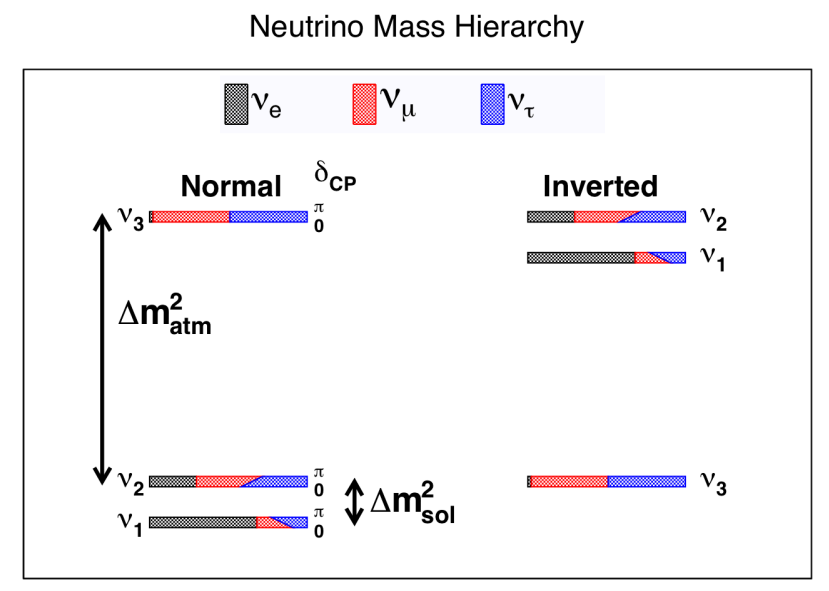

Figure 2.4: Depiction of the neutrino mass hierarchy with the two possible scenarios normal and inverted. Figure from [101].

in the atmosphere and oscillation probabilities are extracted by comparing the up-going and down-going neutrino fluxes. The atmospheric mass splitting is also relevant in accelerator neutrino beams $(L \sim 100 \mathrm{~km}$ and $E \sim 1-100 \mathrm{GeV})$.

At larger $L / E$ where $\Delta m_{21}^{2} L / E \sim \pi$, only the average oscillation probability from $\Delta m_{31}^{2}$ $\left(\Delta m_{32}^{2}\right)$ terms is observed, while experiments instead have sensitivity to $\Delta m_{21}^{2} \cdot \Delta m_{21}^{2}$ is named the solar neutrino mass splitting, in this case named for its relevance in oscillation of neutrinos from the sun. Measurements of oscillation from reactor neutrino sources are also relevant to $\Delta m_{21}^{2}$.

While the oscillation frequency is determined by the mass splittings, the oscillation envelope amplitude is determined by the elements of the PMNS mixing matrix $U$. Its best known experimentally measured values are [6]:

$$
|U|=\left(\begin{array}{ccc}
U_{e 1} & U_{e 2} & U_{e 3} \\
U_{\mu 1} & U_{\mu 2} & U_{\mu 3} \\
U_{\tau 1} & U_{\tau 2} & U_{\tau 3}
\end{array}\right)=\left(\begin{array}{ccc}
0.8 & 0.5 & 0.1 \\
1.5 & 0.6 & 0.7 \\
0.3 & 0.6 & 0.7
\end{array}\right)
$$


$U$ is commonly parameterized

$$
U=\underbrace{\left(\begin{array}{ccc}
1 & 0 & 0 \\
0 & c_{23} & s_{23} \\
0 & -s_{23} & c_{23}
\end{array}\right)}_{\text {Atmospheric }} \underbrace{\left(\begin{array}{cccc}
c_{13} & 0 & s_{13} e^{-i \delta_{C P}} \\
0 & 1 & 0 \\
-s_{13} e^{-i \delta_{C P}} & 0 & c_{13}
\end{array}\right)}_{\text {Cross }} \underbrace{\left(\begin{array}{ccc}
c_{12} & s_{12} & 0 \\
-s_{12} & c_{12} & 0 \\
0 & 0 & 1
\end{array}\right)}_{\text {Solar }}
$$

where $c_{i j}=\cos \theta_{i j}$ and $s_{i j}=\sin \theta_{i j}$. With this parameterization, the atmospheric and solar mixing angles $\left(\theta_{23}\right.$ and $\left.\theta_{13}\right)$ have been separated.

$U$ contains four independent factors: three mixing angles and a phase factor $\delta_{C P}$. Assuming CPT (charge, parity, and time) invariance, the analogous antineutrino oscillation probability is given by 2.10 where the sign of the imaginary term is reversed. $\delta_{C P}$ thus measures the amount of $C P$ violation.

The best-fit values of all oscillation parameters are given in Table 2.2.

Table 2.2: Best-fit oscillation parameters assuming three-neutrino mixing. From [121].

\begin{tabular}{lc} 
Parameter & best-fit \\
\hline$\Delta m_{21}^{2}\left[\times 10^{-5} \mathrm{eV}^{2}\right]$ & $7.37_{-.44}^{+.59}$ \\
$\Delta\left|m_{31}^{2}\right|\left[\times 10^{-3} \mathrm{eV}^{2}\right]$ & $2.56_{-.11}^{+.13}$ \\
$\Delta\left|m_{32}^{2}\right|\left[\times 10^{-3} \mathrm{eV}^{2}\right]$ & $2.54_{-.12}^{+.12}$ \\
$\sin ^{2} \theta_{12}$ & $0.297_{-.47}^{+.57}$ \\
$\sin ^{2} \theta_{23}$ (normal) & $0.425_{-.44}^{+.09}$ \\
$\sin ^{2} \theta_{23}$ (inverted) & $0.589_{-.205}^{+.047}$ \\
$\sin ^{2} \theta_{13}$ (normal) & $0.0215_{-.0025}^{+.0025}$ \\
$\sin ^{2} \theta_{13}$ (inverted) & $0.0216_{-.0024}^{+.0026}$ \\
$\delta_{C P} / \pi$ (normal) & $1.38_{-.38}^{+.52}( \pm 2 \sigma)$ \\
$\delta_{C P} / \pi$ (inverted) & $1.31_{-.39}^{+.57}( \pm 2 \sigma)$
\end{tabular}




\subsubsection{Long-baseline oscillation experiments}

In recent years accelerator-based long baseline experiments such as T2K, MINOS, and NoVA have measuremed $\Delta m_{23}^{2}, \theta_{23}[12][2][9], \Delta m_{13}^{2}$, and $\theta_{13}[11]$. The upcoming experiment DUNE [7], aims to conclusively answer the outstanding questions of the mass hierarchy, the octant of $\theta_{23}$ (i.e. $>$ or $<45^{\circ}$ ), the value of $\delta_{C P}$, as well as test the three-neutrino paradigm.

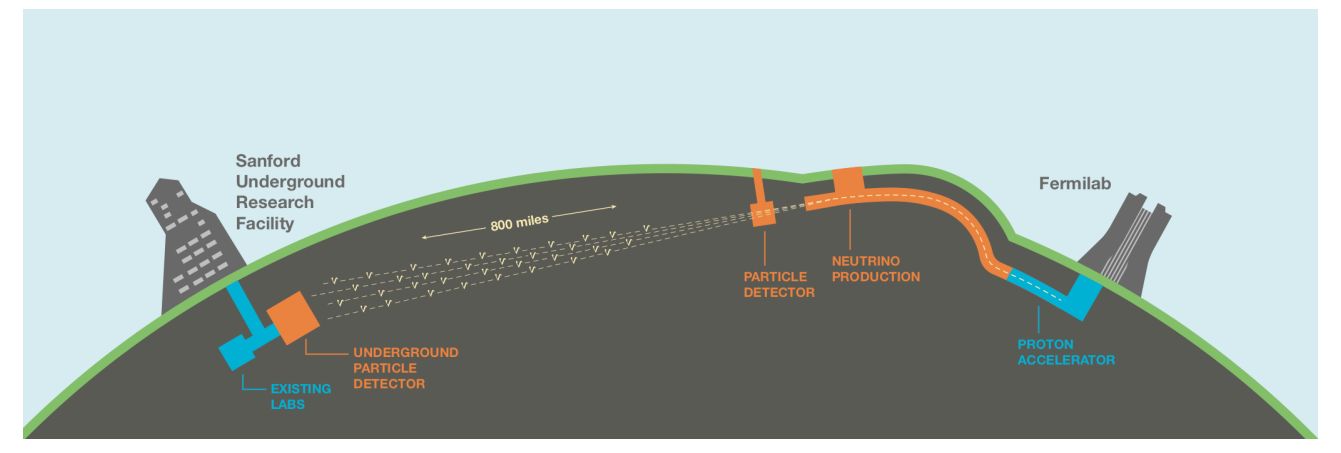

Figure 2.5: Experimental setup for the Deep Underground Neutrino Experiment (DUNE) - a typical long-baseline neutrino experiment design. Figure from [6].

Figure 2.5 shows the upcoming experiment DUNE, which uses a typical long-baseline neutrino experiment design. An accelerator produces a $\nu_{\mu}$ beam with energy $O(\mathrm{GeV})$, and directs it underground to pass through near and far detectors, separated by $O(100 \mathrm{~km})$. The beam energy and the detector sites are chosen so that the far detector location falls as close as possible to the first or second oscillation peak. Accelerator-based oscillation experiment neutrino beams are created using the two-body decay of charged pions, and thus have a broad energy width. Figure 2.6 shows the fluxes for several current beams as well as for DUNE. 


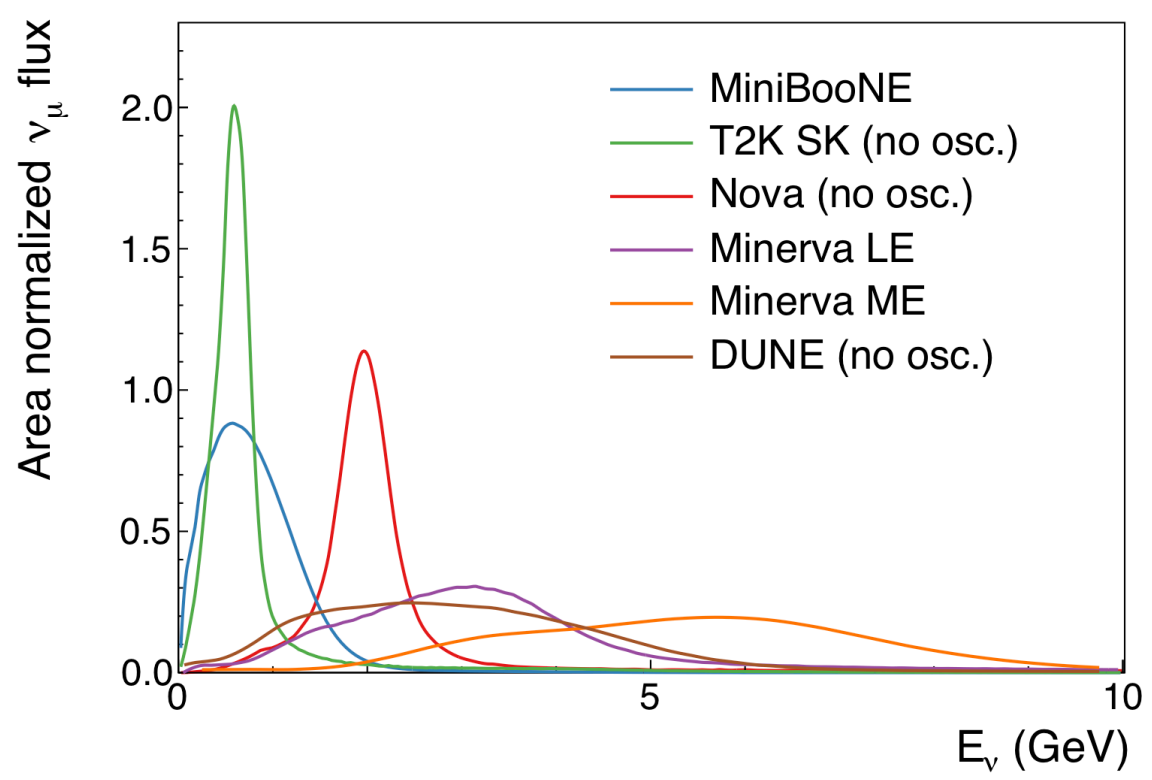

Figure 2.6: Neutrino fluxes for current and future oscillation experiments. Figure courtesy of P. Rodrigues.

Oscillation probability can be obtained by measuring the unoscillated neutrino flux at the near detector and comparing to the remaining $\nu_{\mu}$ and oscillated $\nu_{e}$ fluxes at the far detector. Such measurements are called disappearance and appearance measurements. Though some of the original $\nu_{\mu}$ beam will also oscillate to $\nu_{\tau}$, but beam energies are often too low to create the $\nu_{\tau}$ rest mass. Furthermore, $\nu_{\tau}$ are difficult to detect and detectors must be specially designed to see them.

Using the parameterization of Equation 2.12 and the general oscillation probability of Equation 2.10, the oscillation probability for $\nu_{\mu}$ disappearance for accelerator neutrino scales can be written:

$$
P_{\nu_{\mu} \rightarrow \nu_{\mu}}=1-4\left|U_{\mu 3}\right|^{2}\left|U_{\mu 1}\right|^{2} \sin ^{2} \Delta_{31}-4\left|U_{\mu 3}\right|^{2}\left|U_{\mu 2}\right|^{2} \sin ^{2} \Delta_{32}-4\left|U_{\mu 2}\right|^{2}\left|U_{\mu 1}\right|^{2} \sin ^{2} \Delta_{21}
$$

where $\Delta_{i j}=\Delta m_{i j}^{2} L / 4 E$. Here the imaginary component of Equation 2.10 has vanished because the $U$ terms are completely real. To good approximation remaining terms are proportial to $\sin \left(\Delta m_{12}^{2}\right)$ and can be removed. What remains can be written to resemble 


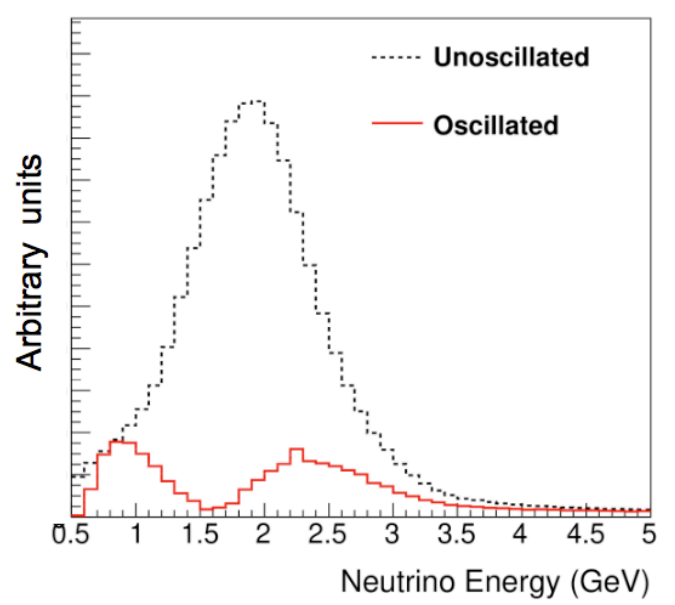

(a) Example far detector event rate, unoscillated compared to oscillated.

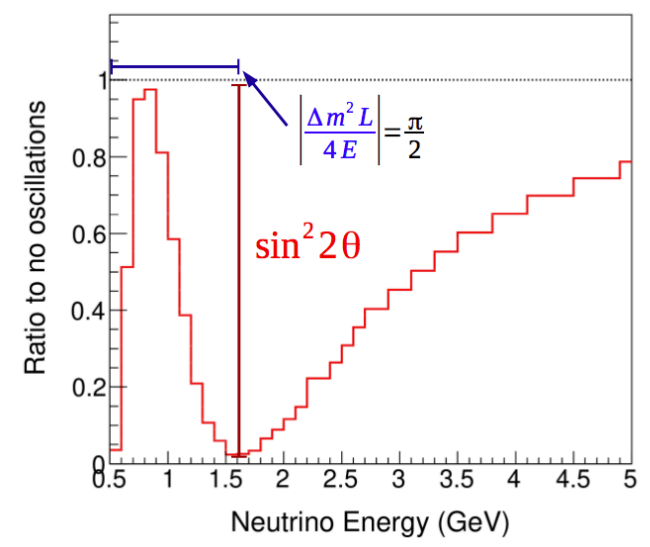

(b) Oscillation probability from $2.7 \mathrm{a}$ showing oscillation amplitude and frequency.

Figure 2.7: Illustration of how oscillation parameters can be determined from far detector spectra. From [127].

oscillation in which only neutrinos participate with a single effective $\Delta m^{2}$ and $\theta$. Reference [92] gives an excellent discussion of the validity and limits of this approximation. The most modern experiments, many of which are seeking to measure $\delta_{c p}$, which is proportional to a (very small) $\sin \theta_{13}$ term, must use the full three-neutrino formula. It is in this way that modern neutrino experiments are forced into precision measurements.

Figure 2.7 illustrates how oscillation parameters might be determined from far detector spectra. The ratio of observed to predicted far detector event rates gives an oscillation probability, and oscillation parameters can subsequently be extracted from the amplitude and frequency. 


\subsubsection{The importance of flux and cross section knowledge to oscillation exper- iments}

DUNE is expected to be completed by 2027. Beyond the earliest stages of data taking, when statistical uncertainties will dominate, large systematic uncertainties threaten DUNE's physics program. Figure 2.8 shows the expected sensitivity to oscillation parameters in $\sigma \mathrm{s}$ as a function of time, making certain assumptions about detector size and operation stability. The green and blue bands represent possible beam designs. The spreads in the bands represent degrees of uncertainties on signal process normalizations, which come from flux and cross section knowledge, as will be explained in this section.

Figure 2.9 shows the systematic error breakdowns for event rates and oscillation parameters for the latest T2K and NoVA oscillation measurements. Note that T2K observes 2-8\% cross section and 3-4\% flux uncertainties on event rates, while NoVA observes 3-5\% cross section uncertainties on event rates, a wide range of cross section uncertainties depending on the oscillation parameter being measured, and very small flux uncertainties. The reason for the disparate flux uncertainties between the two experiments is explained by near and far detector design differences and will be discussed later in this section. 


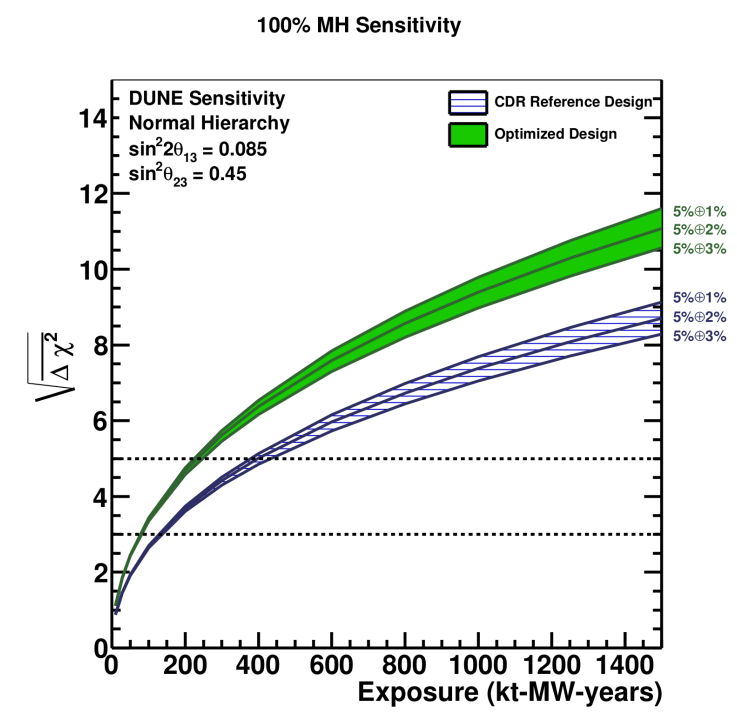

(a)

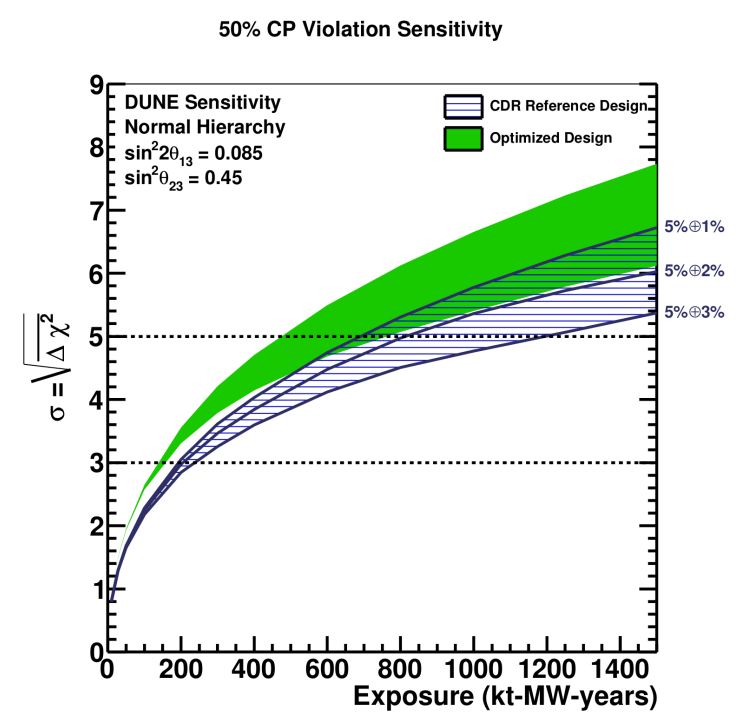

(b)

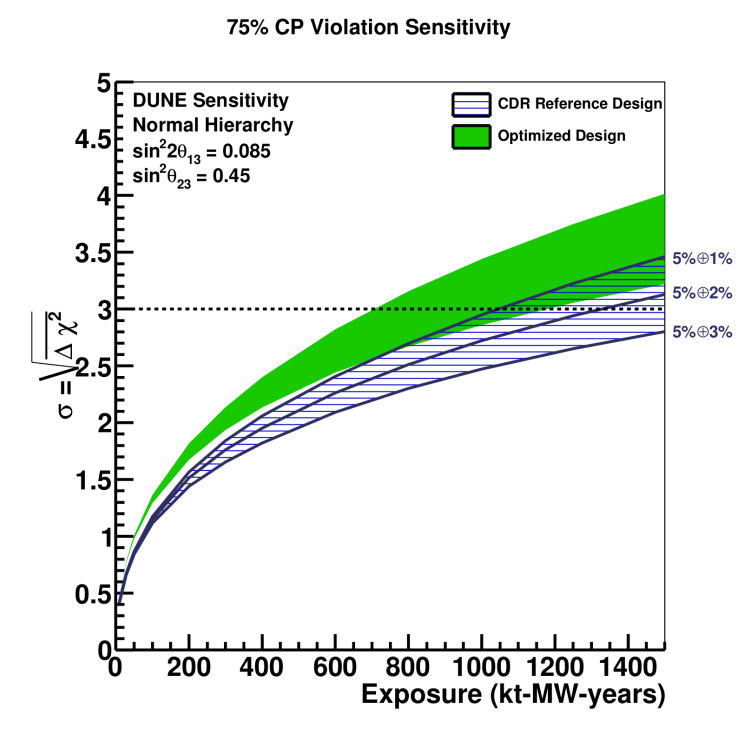

(c)

Figure 2.8: Expected sensitivity to (top) mass hierarchy and CP violation for (bottom left) $50 \%$ and (bottom right) $75 \%$ of $\delta_{C P}$ values as a function of exposure. The green and blue bands represent possible beam designs, and the top, middle, and bottom band lines correspond to a range of values for the $\nu_{e}$ and $\bar{\nu}_{e}$ signal normalization uncertainties, to which $\nu_{\mu}$ cross sections contribute. Figures from [6]. 


\begin{tabular}{lccccc}
\hline \hline Source [\%] & $\nu_{\mu}$ & $\nu_{e}$ & $\nu_{e} \pi^{+}$ & $\bar{\nu}_{\mu}$ & $\bar{\nu}_{e}$ \\
\hline $\begin{array}{l}\text { ND280-unconstrained } \\
\quad \text { cross section }\end{array}$ & 2.4 & 7.8 & 4.1 & 1.7 & 4.8 \\
$\begin{array}{l}\text { Flux \& ND280-constrained } \\
\quad \text { cross section }\end{array}$ & 3.3 & 3.2 & 4.1 & 2.7 & 2.9 \\
$\quad$ SK detector systematics & 2.4 & 2.9 & 13.3 & 2.0 & 3.8 \\
Hadronic reinteractions & 2.2 & 3.0 & 11.5 & 2.0 & 2.3 \\
$\quad$ Total & 5.1 & 8.8 & 18.4 & 4.3 & 7.1 \\
\hline \hline
\end{tabular}

(a) $\mathrm{T} 2 \mathrm{~K}$ far detector event rate systematic errors.

\begin{tabular}{lcccc}
\hline \hline Source & $\nu_{e}$ Signal & $\nu_{e}$ Bkg. & $\bar{\nu}_{e}$ Signal & $\bar{\nu}_{e}$ Bkg. \\
$(\%)$ & $(\%)$ & $(\%)$ & $(\%)$ \\
\hline Cross-sections & $+4.7 /-5.8+3.6 /-3.4+3.2 /-4.2+3.0 /-2.9$ \\
Detector model & $+3.7 /-3.9+1.3 /-0.8+0.6 /-0.6+3.7 /-2.6$ \\
ND/FD diffs. & $+3.4 /-3.4+2.6 /-2.9+4.3 /-4.3+2.8 /-2.8$ \\
Calibration & $+2.1 /-3.2+3.5 /-3.9+1.5 /-1.7+2.9 /-0.5$ \\
Others & $+1.6 /-1.6+1.5 /-1.5+1.4 /-1.2+1.0 /-1.0$ \\
\hline Total & $+7.4 /-8.5+5.6 /-6.2+5.8 /-6.4+6.3 /-4.9$ \\
\hline \hline
\end{tabular}

(b) NoVA far detector event rate systematic errors.

\begin{tabular}{lccc}
\hline \hline Source & $\begin{array}{c}\sin ^{2} \theta_{23} \\
\left(\times 10^{-3}\right)\end{array}$ & $\begin{array}{c}\left|\Delta m_{32}^{2}\right| \\
\left(\times 10^{-5} \mathrm{eV}^{2} / c^{4}\right)\end{array}$ & $\begin{array}{c}\delta_{\mathrm{CP}} \\
(\pi)\end{array}$ \\
\hline Calibration & $+5.4 /-9.2$ & $+2.2 /-2.6$ & $+0.03 /-0.03$ \\
Neutron model & $+6.0 /-13.0$ & $+0.5 /-1.3$ & $+0.01 /-0.00$ \\
Cross-sections & $+4.1 /-7.7$ & $+1.0 /-1.1$ & $+0.06 /-0.07$ \\
$E_{\mu}$ scale & $+2.3 /-3.0$ & $+1.0 /-1.1$ & $+0.00 /-0.00$ \\
Detector model & $+1.9 /-3.2$ & $+0.4 /-0.5$ & $+0.05 /-0.05$ \\
Normalizations & $+1.3 /-2.7$ & $+0.1 /-0.2$ & $+0.02 /-0.03$ \\
ND/FD diffs. & $+1.0 /-4.0$ & $+0.2 /-0.2$ & $+0.06 /-0.07$ \\
Beam flux & $+0.4 /-0.8$ & $+0.1 /-0.1$ & $+0.00 /-0.00$ \\
\hline Total syst. & $+9.7 /-20$ & $+2.6 /-3.2$ & $+0.11 /-0.12$ \\
\hline \hline
\end{tabular}

(c) NoVA oscillation parameter statistical and systematic errors.

Figure 2.9: Latest T2K [4] and NoVA [9] systematic uncertainties on event rates and oscillation parameters. 
DUNE's sensitivity projections are calculated [6] based on flux and cross section uncertainties that are mostly lower than are current oscillation experiments are able to attain. To some extent, these low uncertainties will be feasible by using in situ constraints, but it is also assumed that flux and cross section knowledge will improve [10].

To see how flux and cross section model uncertainties propagate to oscillation parameter measurements, the NoVA experiment can be considered as a specific example. In practice, rather than extracting oscillation parameters as in Figure 2.7, an oscillated far detector prediction is fit to far detector data while floating oscillation parameters. The latest NoVA oscillation analysis of $\bar{\nu}_{\mu} \rightarrow \bar{\nu}_{e}$ appearance uses the minimization of a Poisson negative $\log$-likelihood, $-2 \ln \mathcal{L}$ with unconstrained parameters-of-interest $\Delta m_{32}^{2}, \sin ^{2} \theta_{32}$, and $\delta_{c p}[9]$.

Rather than simply rely on a pure simulation to make the far detector prediction, near detector data is used to constrain the far detector prediction, thereby leveraging the correlations between the spectrums, and ultimately reducing the prediction's systematic errors significantly. In almost all oscillation experiments, near and far detectors are aligned with the same beamline, and flux systematic errors can reliably be reduced. NoVA and DUNE, but not T2K, (will) use functionally identical near and far detectors, made out of the same materials, and only differing in size. This design is extremely desirable, for it also reduces detector and cross section systematics. T2K's near and far detectors are made out of different target materials and operate on different detection principles, so this correlation cannot be used to the same extent. 


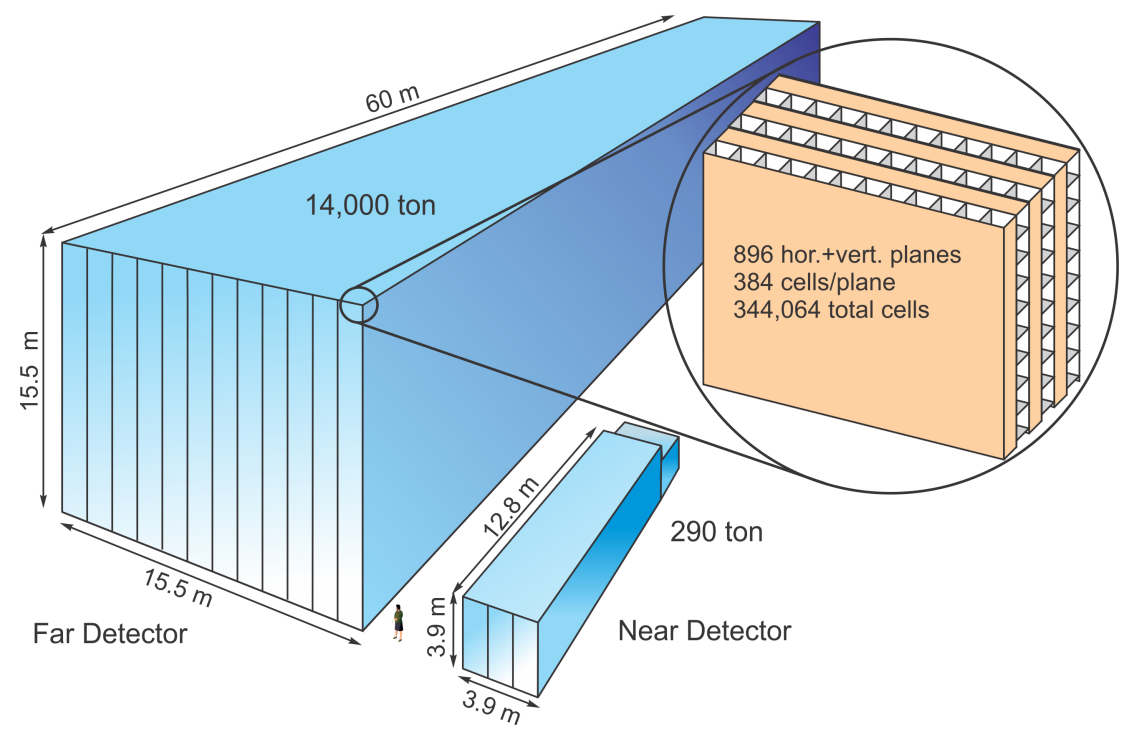

Figure 2.10: The NoVA near and far detectors feature identical geometries, materials, and operation principles, all of which help minimize uncertainties on oscillation measurements. Figure from [43].

Despite these correlations, corresponding systematic uncertainties are not completely removed. In the case of flux, the phase space of neutrino-parent hadrons and thus the neutrino energy spectrum differs slightly at near and far detectors. And size and shape differences of near and far detectors, combined with uncertain beam divergence can subtly change the relative importance of the near and far contributions to the event rate.

Cross section (and more generally, interaction model) uncertainties persist through the data constraint in a different fashion: oscillation probability is a function of a neutrino's true energy, but only a neutrino's interaction products are observed in the detector, so that measured neutrino energy $\neq$ true neutrino energy. And indeed, neutrino beams are not monoenergetic, and they can have a wide $\sim 1-5 \mathrm{GeV}$ spread. Figure 2.11 shows the flux spectrum for several oscillation experiments. 


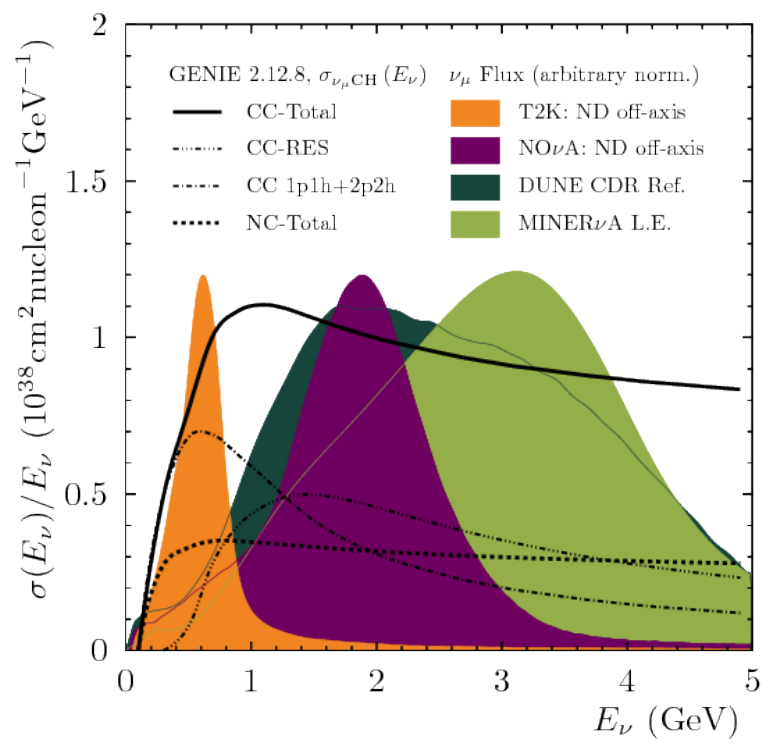

Figure 2.11: Neutrino fluxes for current and future oscillation experiments, including the interaction $\nu_{\mu} \mathrm{CH}$ cross section, broken down by channel, according to event generator GENIE. Figure from [81].

If the interaction model that simulates the observed-to-true energy mapping is incorrect, then the inferred oscillation parameters can also be incorrect. Equivalently, uncertainties in cross sections and interaction models propagate to oscillation parameter fits. To see precisely how cross section uncertainties persist through the data constraint, consider the NoVA far detector prediction extrapolation procedure (shown in Figure 2.12) in more detail. 


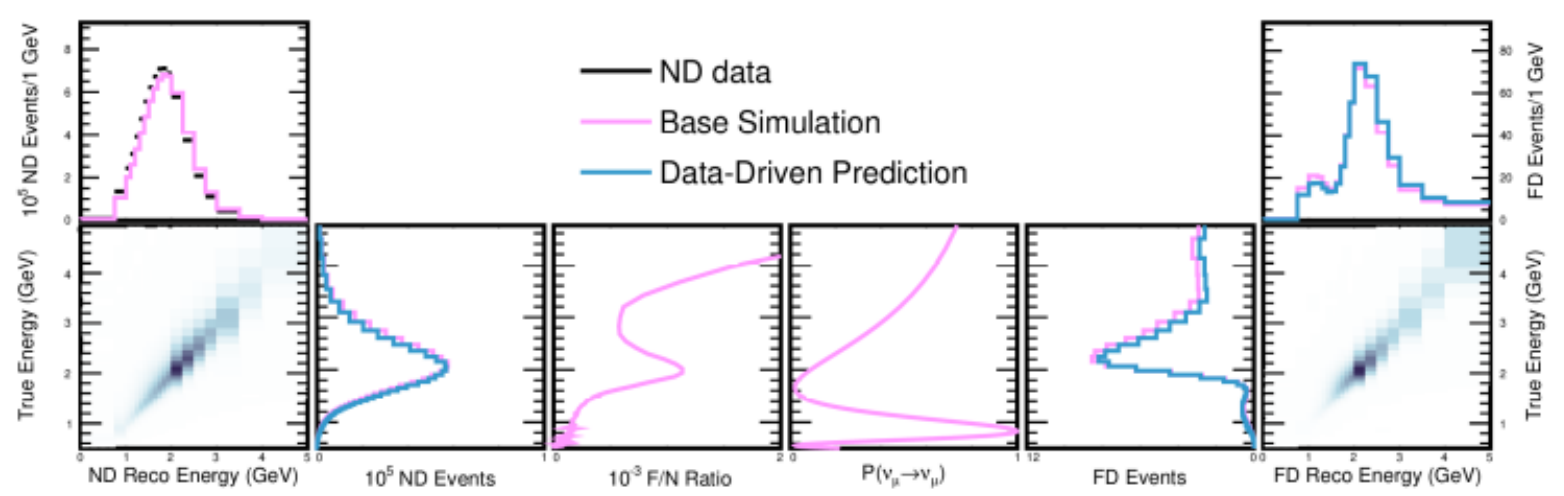

Figure 2.12: NoVA procedure for extrapolating near detector event spectrum to far detector. The procedure begins at the top left with the constraining of the predicted near detector event rate with data. Figure from [128].

The procedure begins (in the top left) by constraining the base model near spectrum prediction with near detector data, and using the interaction model and detector simulations to convert from reconstructed to true energy. Next, beam divergence and oscillation probability (assuming a set of oscillation parameters) convert the prediction to a far detector true energy spectrum. And finally the far detector detector simulation converts from true to reconstructed energy (top right). In Figure 2.12, the effect of the constraint is seen by comparing the base simulation in pink, with the constrained simulation in blue.

There are several difficulties associated with determining true energy from reconstructed energy. As can be seen in Figure 2.11, several interaction channels overlap the operating region of oscillation experiments. These channels must be distinguished in data in order to separate signal from background, and, additionally, analyses often require assumptions about a given event's interaction channel in order to correctly reconstruct its energy.

Interactions are biased by the initial state of the nuclear medium - nuclear binding energy, initial nucleon momenta, nucleon correlations - and product particles from the intial interaction can undergo final state interactions - absorptions, charge exchanges, rescatters, and hadronization. The culmination of all of these effects means that final state particle content does not isolate the initial interaction channel. 


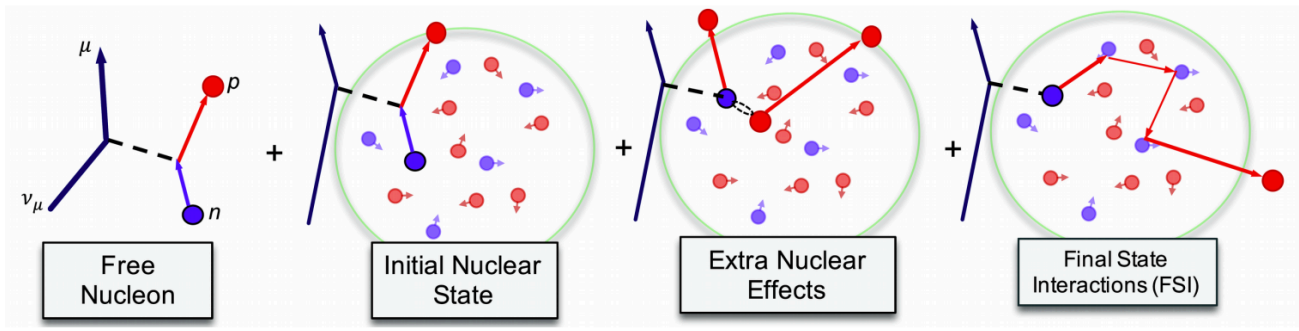

(a)

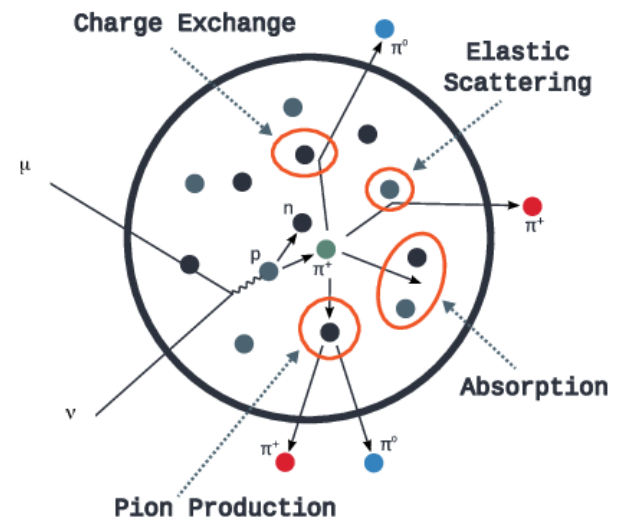

(b)

Figure 2.13: (Top) several nuclear effects obscure the true interaction channel from the observed final states. (Bottom) examples of final state interactions. Figures from [125] and $[26]$. 
Final state interactions can both change final state particle content, potentially causing signal-background misclassification or leading to energy miscalculation with a wrong channel formula. Additionally, final state interactions smear the energies of outgoing particles via intranuclear rescatters.

Ultimately the net effects of flux, cross section, and interaction model errors have myriad and complex effects on the near and far spectra, and the full simulation of Figure 2.12 is relied upon to propagate uncertainties and to determine the entire extent to which uncertainties are reduced by the constraint.

The uncertainties on the constrained far detector prediction are calculated using a version of the many-universe method (described in Section 7.1) in which the analysis is re-performed many times with small changes to the flux and interaction model within their known tolerances, and the spread in the oscillation parameters is taken as the uncertainty. It may be the case that physics processes are missing entirely from interaction models. The same near-far extrapolation procedure can help, to some extent, abate such unknown unknowns. NoVA uses alternate or warped models as fake data, which can be subsequently used to constrain the far detector prediction, and thus evaluate the sensitivity of the constraint to disparate models [127].

\subsection{CHARGED CURRENT NEUTRINO-NUCLEUS INTERACTIONS}

Section 2.2.5 discussed the importance of neutrino-nucleus interaction knowledge to the oscillation physics program. In this section we survey the subject and its current research status.

To calculate neutrino-nucleus scattering from first principles by constructing a nuclear wave function and applying scattering theory is prohibitively difficult. Instead, a complete interaction model typically includes three components: modeling of a initial neutrino-nucleon interaction, modifications due to the nuclear medium, and final state effects on the product particles.

Charged current $(\mathrm{CC})$ interactions (rather than neutral current $(\mathrm{NC})$ interactions) are 
typically used by oscillation experiments as event signal due to the easily-identifiable finalstate charged lepton that tags the neutrino flavor. Fundamental neutrino-nucleon interactions can roughly be categorized by the invariant hadronic mass $W$ event variable, which is defined:

$$
W^{2}=p_{x}^{2}=\left(p_{\nu}+p_{N}-p_{l}\right)^{2}
$$

where $p_{x}$ is the total four-momentum of the final state hadronic recoil system, and $p_{\nu}, p_{N}$, and $p_{l}$ are the neutrino, target nucleon, and final state lepton four momenta. The final state hadron multiplicity increases with $W$. There are three fundamental interactions, summarized in 2.3 .

Table 2.3: Summary of fundamental neutrino-nucleon interaction channels

\begin{tabular}{l|l|l|l} 
Channel & Reaction & $W$ Range $(\mathrm{GeV})$ & Description \\
\hline $\begin{array}{l}\text { Quasi-elastic } \\
\text { QE) }\end{array}$ & $\nu_{l}+N \rightarrow l+N^{\prime}$ & $W \approx m_{N}(1)$. & $\begin{array}{l}\text { The isospin partner of the tar- } \\
\text { get nucleon is ejected from the } \\
\text { nucleus. }\end{array}$ \\
\hline Resonance & $\nu_{l}+N \rightarrow l+N^{\prime}+\pi$ & $\mathrm{m}_{\Delta}(1.2) \lesssim W \lesssim 1.8$ & $\begin{array}{l}\text { Target nucleon is excited to a } \\
\text { baryon resonance, which de- } \\
\text { cays promptly into a nucleon } \\
\text { and pion, usually before exit- } \\
\text { ing the nucleon. See Section } \\
\end{array}$ \\
& & & $\begin{array}{l}\text { 2.1.3. } \\
\text { Neutrino interacts with an in- } \\
\text { dividual quark, breaking up } \\
\text { the nucleus, producing multi- } \\
\text { ple hadrons. }\end{array}$ \\
\hline $\begin{array}{l}\text { Deep inelas- } \\
\text { tic scattering }\end{array}$ & $\nu_{l}+N \rightarrow l+N^{\prime}+X$ & $W \gtrsim 2$ &
\end{tabular}

Figure 2.14 shows the channels' Feynman diagrams and their contributions to the total $\mathrm{CC}$ interaction cross section. Note that in the 1-10 GeV region where oscillation experiments operate, all three interaction channels are active. In particular, the transition region between resonance and DIS is a difficult, but critical region to understand.

In the following sections, each channel is discussed in turn. 

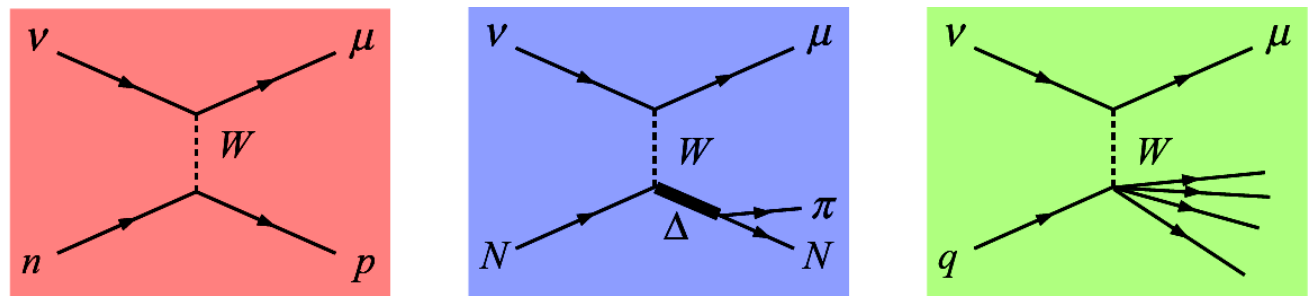

(a) Diagrams of (left) CCQE, (middle) resonant, and (right) DIS.

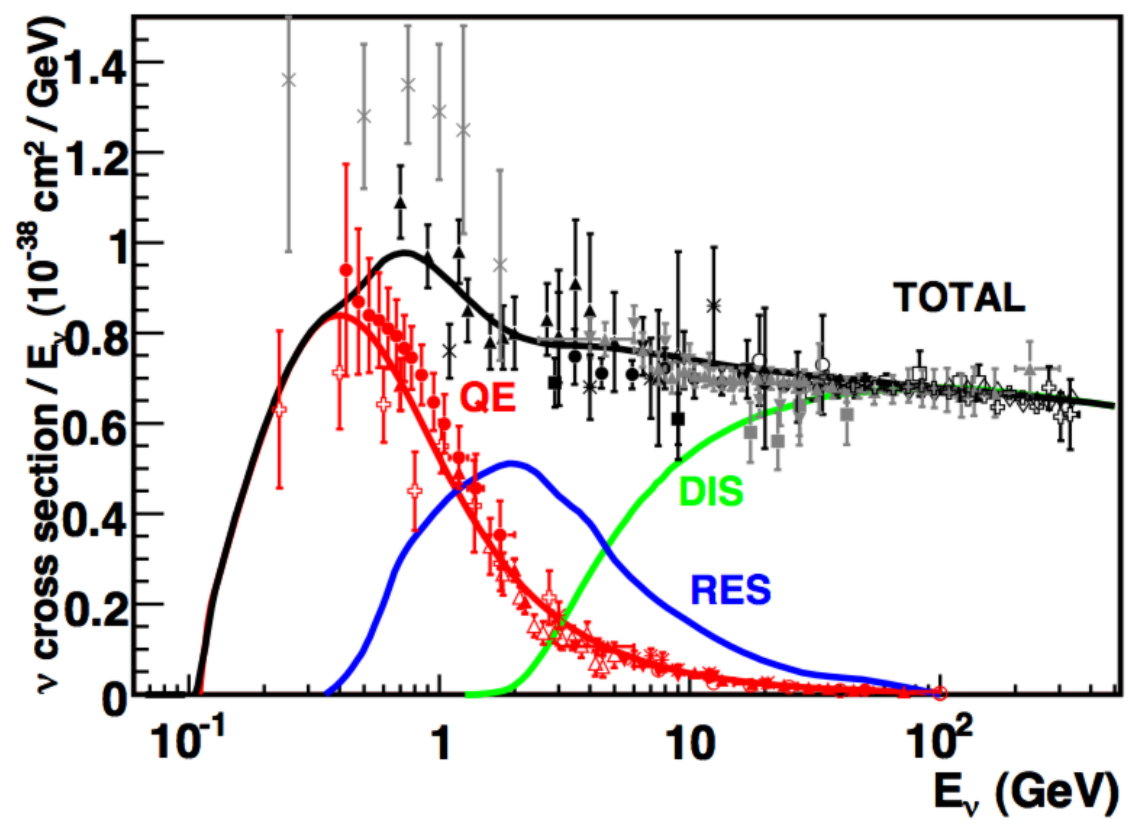

(b)

Figure 2.14: (Top) Neutrino-nucleus charged current scattering interaction channel

Feynman diagrams. (Bottom) Total neutrino-per-nucleon charged current cross section on isoscalar nuclei as predicted by the NUANCE event generator. Total cross section broken down by interaction channels corresponding to 2.14a. Data is from a variety of scattering experiments, and large error bars are due to low statistics and large flux and nuclear uncertainties. Figure from [53]. 


\subsubsection{Quasi-elastic and deep inelastic scattering}

At the lower and upper ranges of $W$, quasi-elastic and deep inelastic scattering dominate.

A charged current quasi-elastic (CCQE) process by itself (without final state interactions) produces no pions and is thus usually a background process for pion measurements. DIS can create many hadrons (including many pions), though at high hadron multiplicities they can be difficult to separate. For this reason DIS is typically characterized by charged lepton kinematics or event-wide variables rather than by exclusive final state hadron kinematics. For pion measurements, DIS can largely by removed by restricting $W \lesssim 1.8 \mathrm{GeV}$.

2.3.1.1 Quasi-elastic scattering formalism The CCQE differential cross section is described by Llewellyn Smith [80] in terms of form factors that attempt to capture the structure of the nucleon:

$$
\frac{\mathrm{d} \sigma}{\mathrm{d} Q^{2}}=\frac{m_{N}^{4} G_{F}^{2}\left|V_{u d}\right|^{2}}{8 \pi\left(p_{\nu} \cdot p_{N}\right)^{2}}\left(A\left(Q^{2}\right) \pm B\left(Q^{2}\right) \frac{s-u}{m_{N}^{2}}+C\left(Q^{2}\right) \frac{(s-u)^{2}}{m_{N}^{4}}\right)
$$

Here, $m_{N}$ is the nucleon mass, $G_{F}$ is the reduced fermi constant $1.166 \mathrm{e}-5 \mathrm{GeV}^{-2}, V_{u d}$ is the transition probability between up and down quarks from the CKM matrix, and $p_{\nu}$ and $p_{N}$ are the momenta of the neutrino and target nucleon. The plus (minus) sign is used for (anti)neutrino scattering. $Q^{2}, s$, and $u$ are the usual Mandelstam variables

$$
\begin{aligned}
Q^{2}=-q^{2}=t^{2} & =-\left(p_{\nu}-p_{l}\right)^{2} \\
s^{2} & =\left(p_{\nu}+p_{N}\right)^{2} \\
u^{2} & =\left(p_{l}-p_{N}\right)^{2}
\end{aligned}
$$

The $A, B$, and $C$ are functions of form factors: two vector form factors $F_{1}\left(Q^{2}\right)$ and $F_{2}\left(Q^{2}\right)$, one axial vector form factor $G_{A}\left(Q^{2}\right)$, and one pseudoscalar form factor $G_{P}\left(Q^{2}\right)$. The Conserved Vector Current (CVC) hypothesis states that strong interactions conserve EM and weak vector currents. From the CVC and vector structure of the EM interaction, the vector form factors can be extracted from electron-nucleon scattering.

The axial vector form factor $G_{A}$ is written with dipole form in terms of an axial mass, $M_{A}$ :

$$
G_{A}\left(Q^{2}\right)=\frac{g_{A}}{\left(1+Q^{2} / M_{A}^{2}\right)^{2}}
$$


where $g_{A}$ can be determined from measurements of the neutron lifetime, and $M_{A}$ can be probed by neutrino scattering experiments with latest measured value [76][35] consistent with $1 . \mathrm{GeV}$.

The pseudoscalar $G_{P}$ appears only in $A$, and it is modified by a factor of $m_{l}^{2} / m_{N}^{2}$, and can be neglected for $\nu_{e}$ and $\nu_{\mu}$ scattering.

2.3.1.2 Deep inelastic scattering formalism The DIS scattering cross section can be written in terms of the Bjorken- $x$ variable characterizing the fraction of momentum carried by the struck quark, as well as by inelasticity $y$, the fraction of neutrino energy that goes into the hadronic system [57]:

$$
\frac{\mathrm{d}^{2} \sigma}{\mathrm{d} x \mathrm{~d} y}=\frac{G_{F}^{2}}{2 \pi} s\left(1+\frac{Q^{2}}{\mathrm{~m}_{W}^{2}}\right)^{-2}\left[x y^{2} F_{1}^{W^{ \pm} N}+(1-y) F_{2}^{W^{ \pm} N} \pm x y\left(1-\frac{y}{2}\right) F_{3}^{W^{ \pm} N}\right]
$$

where

$$
\begin{aligned}
x & =\frac{Q^{2}}{2 m_{N} E_{\text {had }}} \\
y & =\frac{E_{\text {had }}}{E_{\nu}} \\
E_{h a d} & =E_{\nu}-E_{l}
\end{aligned}
$$

The plus (minus) sign corresponds to interactions on (anti)neutrinos. The $F^{W^{ \pm}} N$ are nucleon structure functions dependent on $Q^{2}$ and $x$, and correspond to $W^{ \pm}$interactions on nucleon $N$. By isospin symmetry:

$$
F^{W^{+} p}=F^{W^{-} n}, \quad F^{W^{-} p}=F^{W^{+} n}
$$

The structure functions are proportional to probability densities called parton ${ }^{3}$ distribution functions $f(x)$, which give the probability of finding a quark with four-momentum $p_{i}=x p_{N}$. Structure functions and parton distribution functions can't be calculated from first principles but must be extracted from experiments, e.g. $l-N$ scattering [61][57].

After the initial neutrino-quark interaction, hadronization models such as the phenomenological Lund string model describe the subsequent hadronic shower and work well at invariant hadronic mass $W \gtrsim 2.0 \mathrm{GeV}$.

\footnotetext{
${ }^{3}$ In the words of Griffiths: "partons' - hideous term-[proton constituents] were called in those days, when it was unfashionable to take quarks and gluons too seriously."
} 


\subsubsection{Resonance pion production}

In between the QE and DIS regions, resonance production dominates the neutrino-nucleon interaction cross section. In this process, the target nucleon is excited into a resonance state which, in the operating energy range of oscillation experiments, are the low mass resonances of isospin $1 / 2(N *)$ and $3 / 2(\Delta)$. The resonances de-excite quickly, emitting a pion in the process.

In neutrino interaction models, resonance production is commonly described by ReinSehgal [104]. The differential cross section with respect to $Q^{2}$ and $E_{\text {had }}$, at first assuming a sharply peaked width, is:

$$
\frac{\mathrm{d}^{2} \sigma}{\mathrm{d} Q^{2} \mathrm{~d} E_{\text {had }}}=\frac{G_{F}^{2}}{4 \pi^{2}} \frac{Q^{2}}{|\vec{p}|^{2}} \kappa\left(\frac{m_{N}^{2}-M_{\text {res }}^{2}}{2 m_{N}}\right)\left(u^{2} \sigma_{L}+v^{2} \sigma_{R}+2 u v \sigma_{0}\right) \delta\left(W-M_{\text {res }}\right)
$$

where $\vec{p}$ is the three-momentum of the virtual intermediate vector boson, $M_{\text {res }}$ is the mass of the resonance, $W$ is the observed mass, and

$$
\begin{aligned}
& u=\frac{E_{\nu_{l}}+E_{l}+|\vec{p}|}{2 E_{\nu_{l}}} \\
& v=\frac{E_{\nu_{l}}+E_{l}-|\vec{p}|}{2 E_{\nu_{l}}} \\
& \kappa=\frac{\left(M_{r e s}^{2}-M_{N}^{2}\right)}{2 M_{N}}
\end{aligned}
$$

For finite width, the $\delta$-function becomes a Breit-Wigner factor:

$$
\delta\left(W-M_{\text {res }}\right) \rightarrow \frac{1}{2 \pi} \frac{\Gamma}{\left(W-M_{\text {res }}\right)^{2}+\Gamma^{2} / 4}
$$

where $\Gamma$ is the resonance width.

$\sigma_{L}, \sigma_{R}$, and $\sigma_{0}$ are partial cross sections for a resonance creation from an intermediate boson of polarization left-handed, right-handed, or zero helicity. They are:

$$
\begin{array}{r}
\sigma_{L}\left(Q^{2}, E_{\text {had }}\right)=\frac{\pi M_{\text {res }}}{2 \kappa M_{N}} \sum_{\text {spins }}\left|f_{ \pm}\right|^{2} \\
\sigma_{0}\left(Q^{2}, E_{\text {had }}\right)=\frac{\pi M_{N}}{2 \kappa M_{\text {res }}}\left(\frac{p^{2}}{Q^{2}}\right) \sum_{\text {spins }}\left|f_{0}\right|^{2}
\end{array}
$$

Here, $f_{p m}$ and $f_{0}$ are helicity amplitudes. They contain the interaction dynamics and are given by the relativistic quark model of Feynman, Kislinger, and Ravndal [49], in which 
hadrons are modeled as a system of quarks coupled as relativistic harmonic oscillators. The helicity amplitudes are a function of dipole-form vector and axial vector form factors $G^{A}$ and $G^{V}$

$$
G^{A, V}\left(Q^{2}\right)=\left(1+\frac{Q^{2}}{4 M_{N}^{2}}\right)^{1 / 2-N}\left(\frac{1}{1+Q^{2} / M_{A, V}^{2}}\right)^{2}
$$

where $N$ is the number of oscillator quanta in the final resonance and masses $M_{V}^{\text {res }}=0.84 \mathrm{GeV}$ and $M_{A}^{\text {res }}=1.12 \mathrm{GeV}$ are from neutrino-nucleus cross section data [75].

The Rein-Sehgal calculation considers 18 resonances for observed invariant mass $W<$ $2.0 \mathrm{GeV}$. Interference between resonances with identical spin and orbital angular momenta are considered. The final state $\mu^{-} n \pi$, for example is created as the result of contributions from $\Delta(1232), \Delta(1920)$, and $\mathrm{N}(1720)$.

Direct non-resonant, so-called "background" processes can also produce pions, and in

order to improve agreement with data, a nonresonant isospin $\frac{1}{2}$ process is added incoherently by R-S. In reality, resonant and non-resonant contributions to the same final state interfere with each other and they may shift the apparent location of the resonance peak. Event generators using R-S for resonance production and a DIS model as described in Section 2.3.1 must take care not to double count. DIS hadronization models do not perform as well at regions below $W=2$. Only very recent work by Kabirnezhad [68] extends R-S and attempts to account for the interferences, which may be probe-able by experiment.

\subsubsection{Coherent pion production}

Coherent pion production is another, subdominant ( $\sim 1 \%$ of total CC interaction rate) fundamental neutrino-nucleus interaction. In this interaction, depicted in Figure 2.15, a pion is produced without modifying the nucleus. To preserve the nucleus, the square of the four-momentum exchanged with the nucleus $|t|$ is small, and the particle exchanged with the nucleus does not go on shell. Coherent pion production is described by another model from Rein-Sehgal [105][106].

This model makes use of the partially-conserved axial vector current (PCAC) theorem [14] which considers a neutrino interaction with no four-momentum transfer to the target, i.e. $Q^{2}=0$. The PCAC theorem relates the target and outgoing hadronic final states of a $Q^{2}=0$ 
neutrino scatter to a pion scatter off the same target and producing the same hadronic final state. Thus the neutrino interaction cross section can be calculated from $\pi N$ or $\pi A$ scattering experiments.

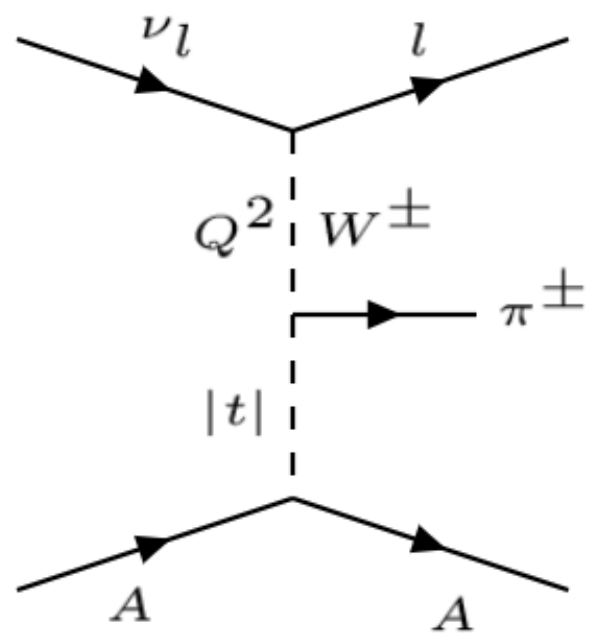

Figure 2.15: CC coherent pion production Feynman Diagram. $|t|$ is the four-momentum exchanged with the nucleus $A$.

\subsubsection{Modifications to the nuclear medium}

The previous sections have described fundamental neutrino-nucleon interactions. In reality, the target nucleon is embedded in the nucleus, which can modify the interaction. We consider these nuclear modifications in this section.

Until very recently, the target nucleus had been modeled as a relativistic Fermi gas (RFG) with no intranuclear effects, and neutrino interactions were modeled in the impulse approximation, i.e. as an incoherent sum of instantaneous $\nu-N$ interactions. In 2009 the MiniBooNE experiment published the first high statistics measurement of CCQE neutrino scattering on $\mathrm{CH}$ and saw a large excess of data compared to prediction in the low four momentum squared $Q^{2}$ region [17].

Since then, this discrepancy has come to be explained in large part by the lack of certain 
nuclear effects in neutrino interaction models. Inspired in large part by analogous work in electron scattering (see for example [56]), many important nuclear effects were added which bring models into agreement with data.

Figure 2.16 shows the MiniBooNE CCQE cross section as a function of neutrino energy in data and compared to models with a treatment of the nucleus more sophisticated than the RFG.

\section{CCQE on ${ }^{12} \mathrm{C}$}

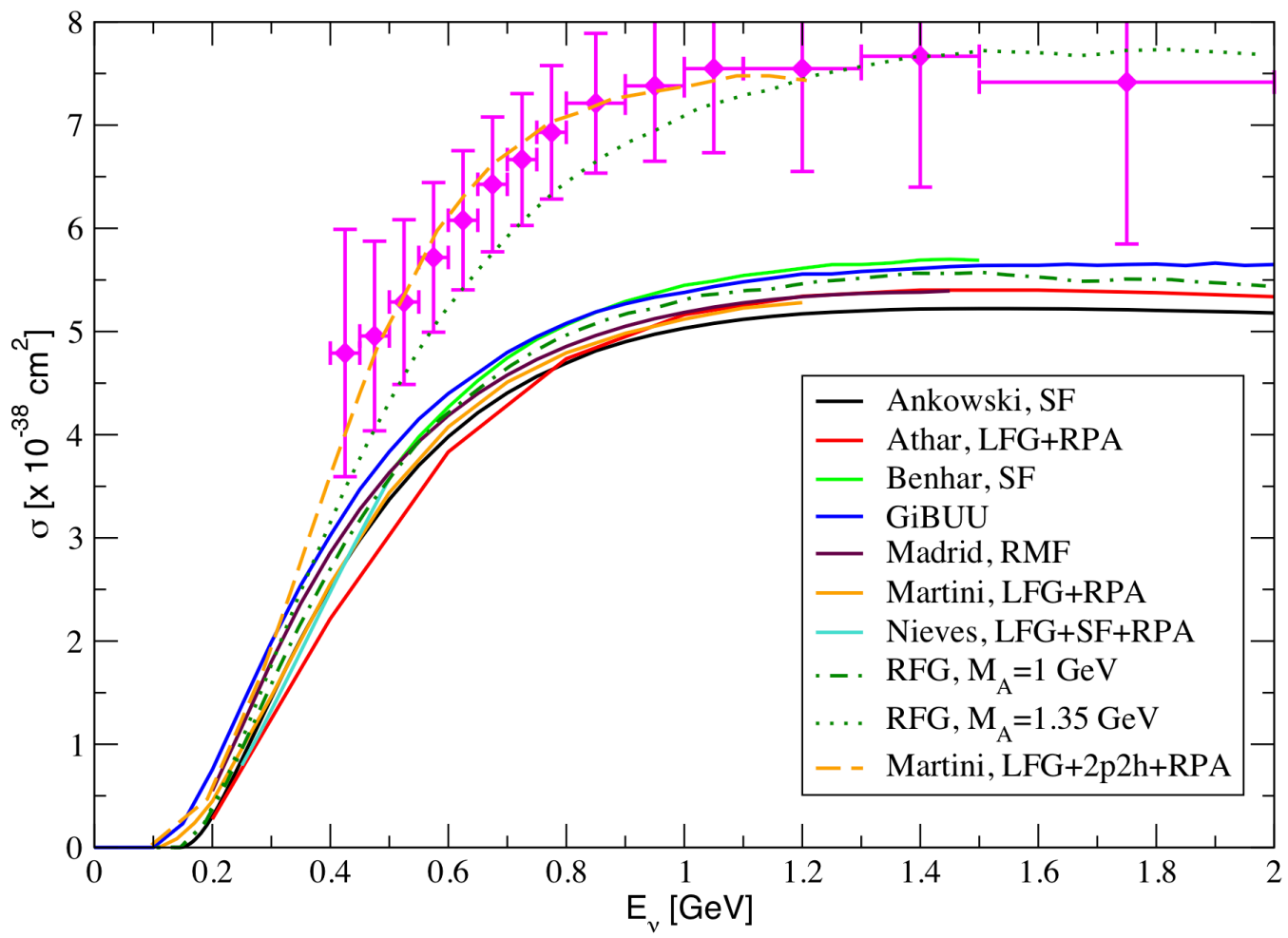

Figure 2.16: MiniBooNE CCQE $\nu_{\mu}$ cross section data and several predictions with alternate nuclear models. Figure from [25].

Of the two models that agree best with the data, the green dotted line uses a default RFG model but with the CCQE axial mass $M_{A}^{\mathrm{CCQE}}$ tuned well above the world average at 1. GeV. The orange dashed model, on the other hand, considers several nuclear effects, but critically it includes a so-called two-particle two-hole $(2 \mathrm{p} 2 \mathrm{~h})$ model. A $2 \mathrm{p} 2 \mathrm{~h}$ process is one in which the intermediate boson interacts with a correlated pair of nucleons, which are both 
ejected from the nucleus (in the absence of final state interactions). The following sections discuss the base RFG model, $2 \mathrm{p} 2 \mathrm{~h}$ processes, and an another important category of effects called random phase approximation (RPA) effects.

2.3.4.1 Relativistic Fermi gas nuclear model The most common model of the nucleus has been the relativistic Fermi gas model by Smith and Moniz [116]. Here, nucleons are considered non-interacting fermions (only interacting with the mean field of the nucleus) confined to a potential well with walls at the nuclear radius, and binding energy $\sim 30 \mathrm{MeV}$. These assumptions are effective at high $Q^{2}$, and there, the model agrees well with data. The nucleon momentum distribution is modeled with a flat distribution, with uniform density through the nucleus, and at a value below the Fermi cutoff momentum $k_{F} \sim 250 \mathrm{MeV}$.

In an RFG, the lowest energy levels are filled, and due to the Pauli exclusion principle, ejected nucleons must have momentum greater than $k_{F}$. This effect is called Pauli blocking, and it, together with nuclear binding, reduce the rate of interactions at low four-momentum transfer $Q^{2}$.

Some modifications and alternatives to the Smith-Moniz RFG have been developed. Bodek and Ritchie have added a high tail to the momentum distribution to account for the fact that interacting nucleons can result in one nucleon with momentum greater than $k_{F}$ [37]. A local fermi gas model modifies the uniform nucleon momentum distribution to instead depend on a nucleon's position within the nucleus [96]. Spectral function models by Benhar et al. provide probability distributions of nucleons with certain momentum and removal energy [30]. Ultimately, still more sophisticated nucleon interaction modifications were needed to agree with experiment. These are discussed in the next section.

\subsubsection{Multi-nucleon correlations — random phase approximation (RPA) and} Multi-nucleon $\boldsymbol{n p n h}$ processes Long-range correlations between nucleons within the nucleus are modeled by the random phase approximation, which is a weak force analogue to the screening of an electric charge in a dielectric. In this case, a mediating $Z$ or $W$ boson polarizes the nuclear medium, and the electroweak coupling is modified by the presence of strongly interacting nucleons. RPA has been implemented into neutrino scattering interac- 
tion models for example by Nieves et al. [96]. When added to a RFG, RPA effects naturally suppress the interaction rate, and the effect is strongest at low $Q^{2}$, falling to zero as $Q^{2}$ grows.

While RPA ultimately modifies a neutrino interaction with an individual nucleon, multinucleon effects address interactions between pairs or groups of nucleons.

It is known from nuclear physics that a nucleon in carbon is involved in a short range correlation (SRC) with another nucleon (wherein their wave functions overlap at small distances) about $20 \%$ of the time [46]. In $90 \%$ of those cases, the correlation is an $n p$ pair [119]. In neutrino scattering a correlated $n p$ pair is ejected from the nucleus as two protons (one spectator and other transformed by the weak process), leaving two holes in the nucleus. As was seen in Figure 2.16, this so-called 2p2h process can have a large effect on a "CCQE" measurement interaction rate, where quotation marks are added to distinguish this process from a pure CCQE process. Figure 2.17 illustrates a 2p2h process, compared with the usual $1 \mathrm{p} 1 \mathrm{~h}$ process.
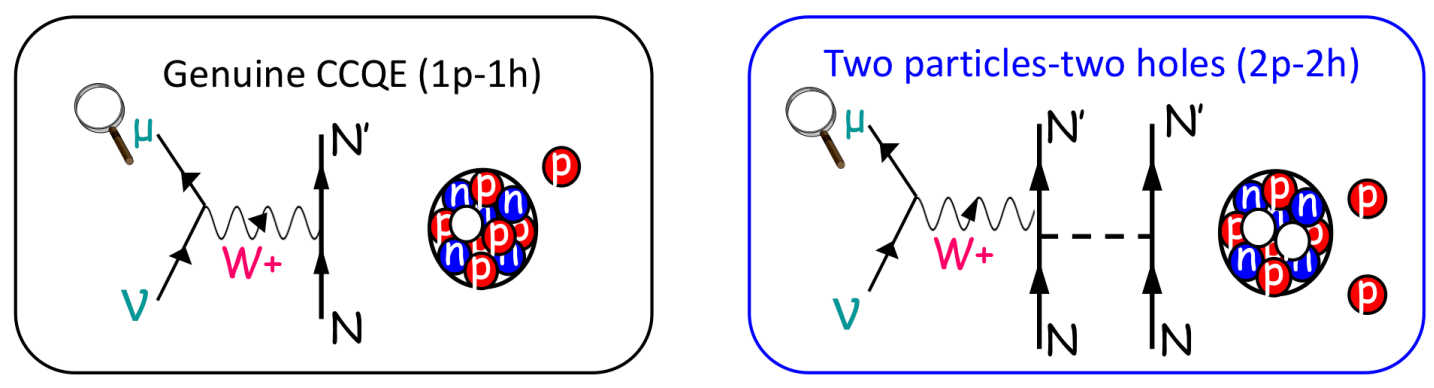

Figure 2.17: Depictions of 1p1h and 2p2h processes. Figure from [70].

Short range correlations are strong in nature and can greatly increase the relative momentum of the nucleons. Modifying the momentum distribution with a high-end tail as described in Section 2.3.4.1 can account for this, but it fails to eject a second nucleon.

Instead, first-principle models of $2 \mathrm{p} 2 \mathrm{~h}$ phenomena have been developed. The meson exchange current (MEC) is one such important phenomena, wherein a nucleon pair interacts via the exchange of a virtual pion. In MEC formulations four Feynman daigrams of the process are considered [124] (Figure 2.18). 


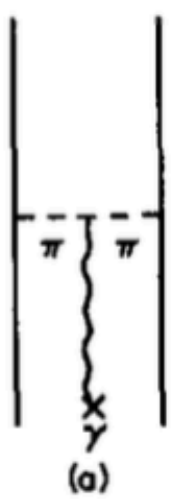

,

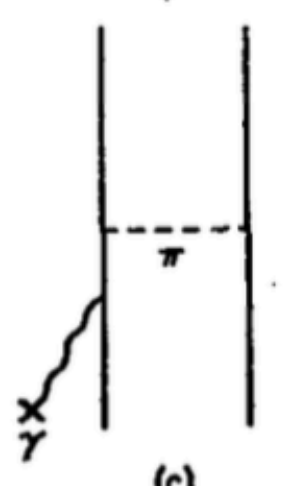

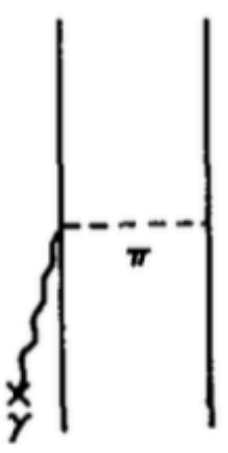

(b)

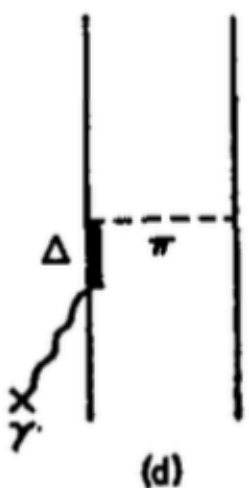

Figure 2.18: MEC Feynman diagrams for electron scattering. In the analogous CC weak process, the photon is replaced by a $W$. Figure from [124].

MECs enhance the interaction rate primarily at low $Q^{2}$. They contribute to the pure CCQE interaction cross section peak as well as to the dip between the CCQE and first resonance peak. Together with RPA, which decreases the cross section at low energies, "the final picture is that of a delicate balance between a dominant single nucleon scattering, corrected by collective effects, and other mechanisms that involve directly two or more nucleons" [97].

$\mathrm{RPA}$ and $2 \mathrm{p} 2 \mathrm{~h}$ effects are a very active and relatively recent area of research in neutrino scattering. As measurements are more carefully designed to probe these processes, analyzers have switched from claiming to report pure processes such as "CCQE" (as in Figure 2.2c) or "resonance pion production" (Figure 2.3), to reporting in terms of final state particles, e.g. CC $0 \pi$ or $\mathrm{CC} 1 \pi$. 
$N . b$. these effects have been studied primarily as modifications to $0 \pi$ final states. It is possible, for example for one of a $p n$ correlated pair may be excited to a resonance that results in a pion final state. Generally it is not known whether such effects may influence resonance production and pion final states.

\subsubsection{Nuclear final state interactions (FSI)}

After the initial neutrino interaction with the nuclear medium, product particles from the interaction are subject to FSIs. Final state particles can undergo elastic or inelastic scatters, charge exchange, or absorption (as pictured in Figure 2.13b). Such final state make it difficult to reconstruct the nature of the initial interaction for they both smear the kinematic event reconstruction, and they transform the particles leaving the initial interaction into a different set of particles which are observed leaving the nucleus.

Final state interactions are difficult to study and model. The nucleus is a many body system subject to the strong force, and its effects are too difficult to calculate from first principles. Instead, there are three current approaches to modeling the problem. The first and simplest approach uses nuclear scattering cross sections, for example of $p A$ or $\pi A$, to scatter product particles. A-scaling assumptions can shift the interactions to other nuclei, and isoscalar symmetries can be used to convert to $\mathrm{n}$ and $\pi^{0}$. This approach of course suffers from the inaccuracies of the $A$-scaling assumptions and from the fact that nuclear scattering data originates from the outside of a nucleus, instead of the inside.

A second approach is called a hadronic cascade. In this method, product particles are stepped through the nucleus by some fraction of the nuclear radius. At each step, a probability of interacting or simply continuing to propagate is given, and interaction avenues can include the usual processes, like absorption, charge exchange, etc. Interaction probabilities may be either extracted from data or determined from first-principles.

A final, more first-principles approach uses the classical Boltzmann-Uehling-Uhlenback equation [122], which subjects each particle to a time evolution function of its phase space density.

As the lightest mesons, pions are particularily well-suited to probe FSI effects. Pion 
absorption, for example can be studied by searching for left-right asymmetry between the outgoing lepton and proton [39].

\subsection{PREVIOUS MEASUREMENTS OF $\nu_{\mu}$ CC SINGLE CHARGED PION PRODUCTION}

Datasets of $\nu_{\mu} \mathrm{CC} 1 \pi^{+}$exist from the Argonne National Lab hydrogen bubble chamber (1982) [102] and the Brookhaven National Lab deuterium bubble chamber (1986) [71]. These datasets are difficult to compare to modern experiments. First, modern detectors are built from more complex materials and it is not known how to extrapolate these low- $A$ cross sections to higher mass. And second, they feature large uncertainties due to low statistics and poor flux modeling. A recent re-analysis of these data attempted to remove the measurements' flux dependences, and the data were found to be in agreement [126]. This reanalysis was further used to improve interaction model parameters [112].

Another measurement from ANL [42], as well as more recent measurements from K2K [113] and MiniBooNE [16] measured CC $\pi /$ CCQE cross section ratios on some heavier targets.

The next generation of absolute cross section measurements had improved statistics (thousands of events) and smaller flux uncertainties (5-15\%). These include measurements from MiniBooNE on a hydrocarbon mineral oil (2010, [18]), MINERvA on hydrocarbon plastic $\left(2015,\left\langle E_{\nu}\right\rangle \sim 4 \mathrm{GeV}\right)$ [44], and T2K on water (2017) [3]. These measurements showed varying degrees of disagreement in shape and normalization, and the MINERvA and MiniBooNE measurements in particular could not be brought into agreement[117].

Other recent work attempts to fit pion production parameters of the GENIE [27] event generator's interaction model to the ANL and BNL data and MINERvA measurements across all pion production channels $\left(\nu_{\mu} \mathrm{CC} 1 \pi^{+}\right.$[44], $\nu_{\mu} \mathrm{CCN} \pi^{+}$[84], $\nu_{\mu} \mathrm{CC} 1 \pi^{0}$ [24], $\left.\bar{\nu}_{\mu} \mathrm{CC} 1 \pi^{0}[77]\right)$. The conclusion of this work is that it: "improves the GENIE production model significantly, but strong tensions remain" [118].

The very latest measurements of the $\mathrm{CC} 1 \pi^{+}$channel are from ArgoNeuT on Argon (2018, [8]) and from T2K on hydrocarbon plastic (2019, [5]). These measurements have 
adopted the more topological and agnostic signal definitions (which were mentioned in the context of CCQE and nuclear effects in 2.3.4.2), that are defined primarily by the mere presence of a final state charged pion. Additionally, these measurements make explicit effort to minimize the contamination by model dependences. There are reasons to believe that model dependence contaminations and conflicting nuanced analysis decisions could explain the disagreements between MiniBooNE and MINERvA [33]. 


\subsection{NUMI NEUTRINO BEAM}

Fermilab's NuMI (Neutrinos at the Main Injector) neutrino beam is a $\nu_{\mu}$ and $\bar{\nu}_{\mu}$ source that

provides neutrinos to the MINERvA, MINOS, and NoVA detectors for oscillation and cross section measurements.

NuMI neutrinos are created from the decays of charged mesons, in particular pions and kaons, which are products of collisions between a $120 \mathrm{GeV} / c$ proton beam and a carbon target rod.

NuMI is an intense beam, capable of continuously directing 60 billion muon neutrinos through the MINERvA detector every second during normal operation (circa 2017). The beam features a broad spread of neutrino energies between 0 and $20 \mathrm{GeV}$. Between 2005 and 2013, the beam ran in a low energy (LE) configuration with a neutrino energy peak around $3.5 \mathrm{GeV}$. Since then, the beam has been operating in its medium energy (ME) configuration with neutrino energy peak near $7 \mathrm{GeV}$. The LE and ME predicted fluxes are shown in Figure 3.1.

In neutrino mode, NuMI is composed of predominently muon neutrinos with small muon antineutrino $(\sim 5 \%)$ and electron neutrino/antineutrino componenents (total $<1 \%)$.

In Sections 3.1 and 3.2 the NuMI beamline design and simulation are presented. 


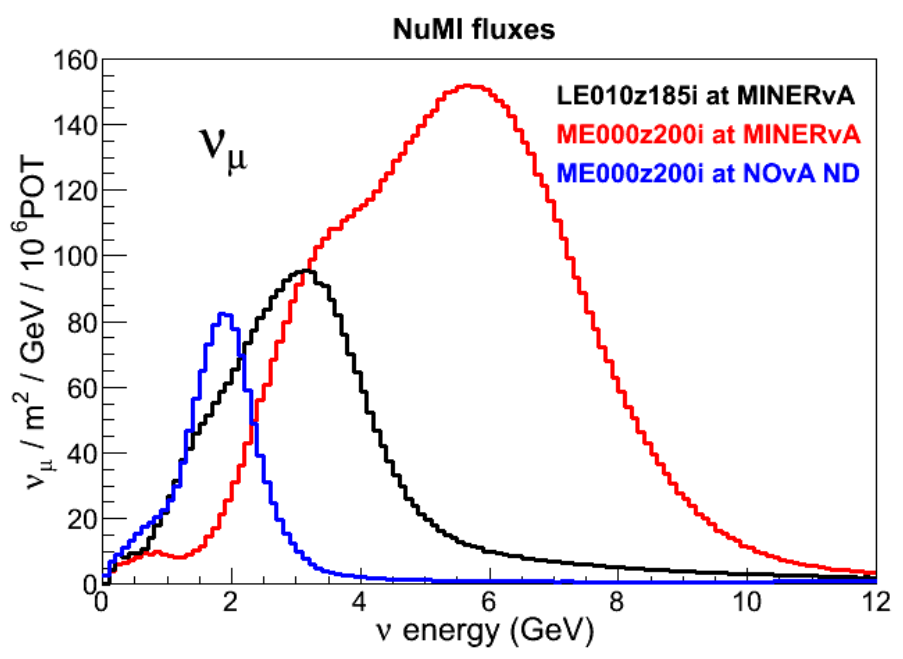

Figure 3.1: Simulated fluxes for the LE and ME runs in the MINERvA and NoVA detectors. Figure from [19].

\subsection{DESIGN}

This section follows the creation of the neutrino beam, step-by-step, from the initial proton beam (3.1.1), to the proton-target collisions and hadron production (3.1.2), the magnetic focusing horns that focus the hadrons (3.1.3), and finally to the decay pipe where the hadrons decay into neutrinos (3.1.4).

\subsubsection{Main Injector proton beam}

The process to create the proton beam begins by accelerating $\mathrm{H}^{-}$ions to $750 \mathrm{keV}$ using a radio frequency quadrupole. The ions are then passed to a linear accelerator, which accelerates them to $400 \mathrm{MeV}$ and then directs them through a thin carbon foil to strip the $\mathrm{H}^{-} \mathrm{s}$ of their electrons, leaving only protons.

The protons are passed to the 75 m-radius Booster synchrotron, where they are accelerated to $8 \mathrm{GeV} / c$ in less than 67 ms over the course of $\sim 40,000$ laps. At $8 \mathrm{GeV} / c$, the Booster can fit one $1.6 \mu$ s-wide batch of protons. 


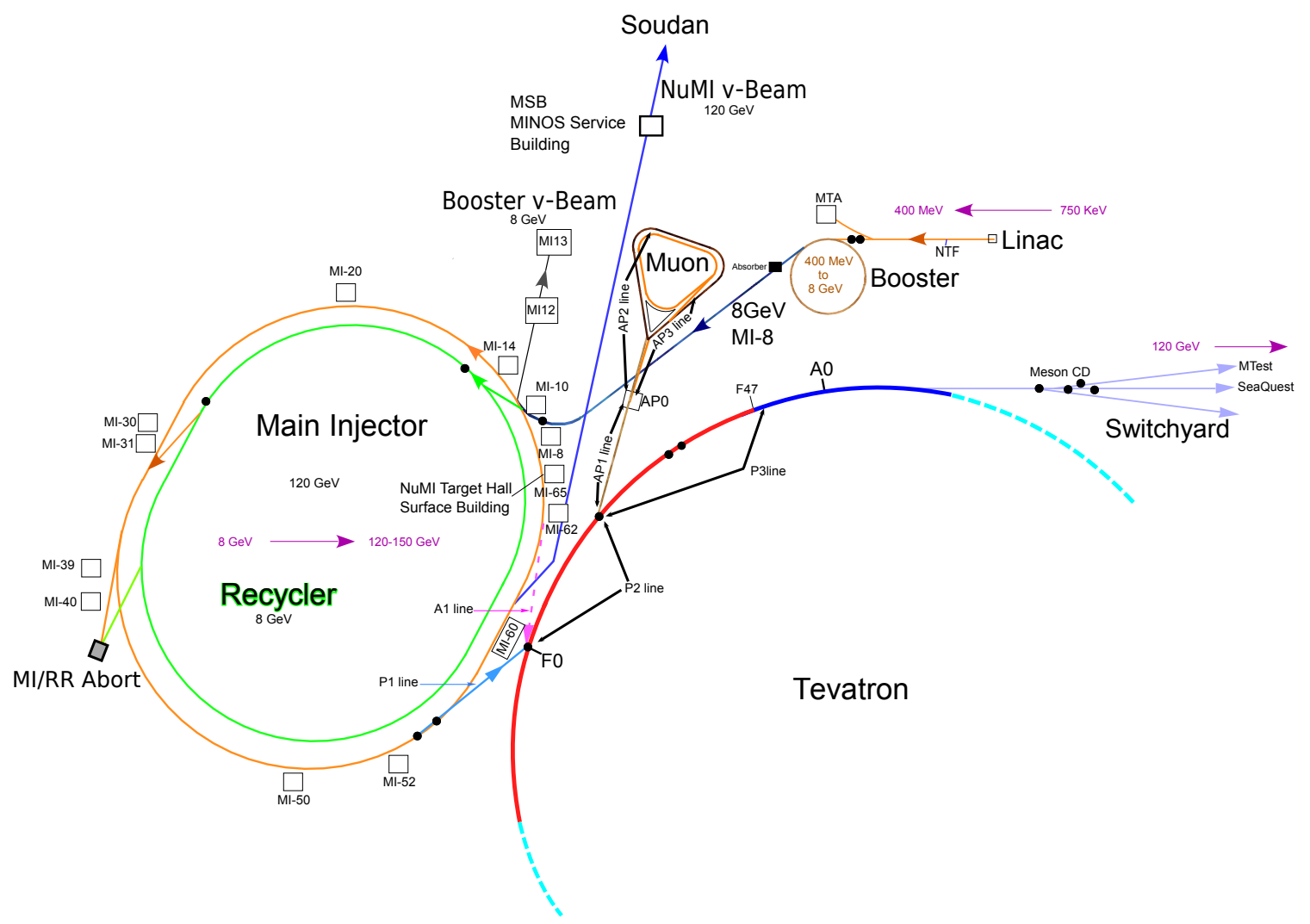

Figure 3.2: Overview of the Fermilab accelerator complex. Figure from [13].

Protons are next passed to the Main Injector synchrotron ring which accelerates the protons to $120 \mathrm{GeV} / c$. The Main Injector has a circumference seven times that of the Booster, and thus it can fit seven proton Booster batches. One batch space is used for proton injection, and thus the Main Injector fits six Booster batches. With a technique called slip-stacking, an up to six additional batches (for a total of 12) can be passed to the Main Injector by "slipping" two batches into one batch space.

After slip-stacking and acceleration to $120 \mathrm{GeV} / c$, the proton batches leave the Main Injector ring in a spill, directed toward the NuMI Target Hall. By the end of the ME run, NuMI steadily upgraded to full $6+6$ slip-stacking, with a spill intensity of more than 5e13 protons, and spill separation of $1.33 \mathrm{~s}$. 


\subsubsection{Target}

The proton beam leaving the Main Injector is directed $3.3^{\circ}$ downward and travels $350 \mathrm{~m}$ to the underground Target Hall. The NuMI components from the target onward are depicted in Figure 3.3. At the Target Hall, the beam is incident on the NuMI target with a nearly circular cross sectional spot of $1.4 \mathrm{~mm}$ diameter. The ME-era target (shown in Figure 3.4) is a $1.2 \mathrm{~m}$-long rod, consisting of 48 rectangular graphite segments, each $24 \mathrm{~mm}$ long and with $7.4 \times 63 \mathrm{~mm}^{2}$ cross section. In total, the $1.2 \mathrm{~m}$ length corresponds to 2.5 nuclear interaction lengths.

There are several important considerations in target design. In particular, a longer target increases the neutrino yield, but can result in a critically hot target and more hadron reinteractions, which are not as well understood. For this reason, the target cross section must be kept small so that most hadrons exit out the side before reinteractions.

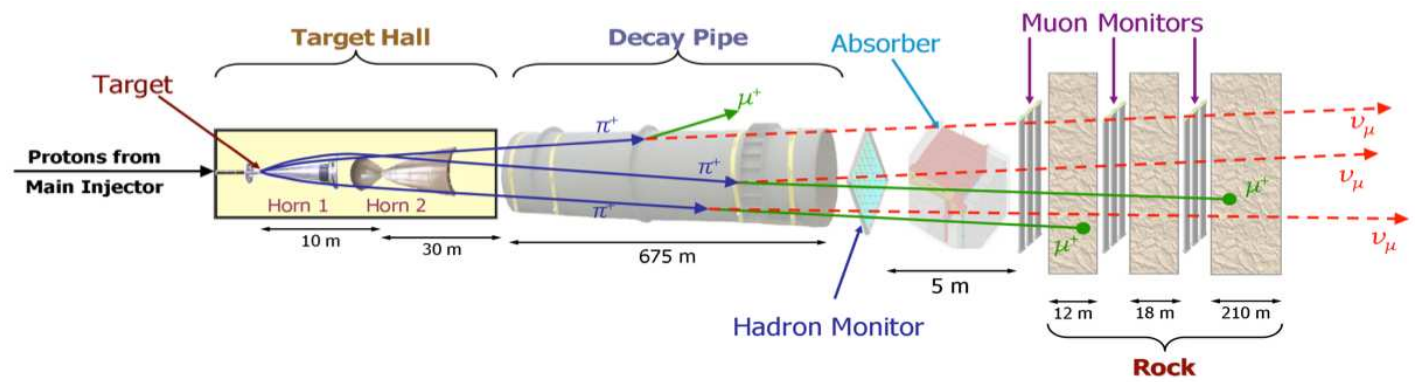

Figure 3.3: Schematic of the NuMI components. Figure from [13].

The target is surrounded by two stainless steel water-cooling pipes, all of which are enclosed in an aluminum alloy container filled with gaseous helium. The target structure and downstream components are protected from a mis-steered proton beam by a $1.5 \mathrm{~m}$ long graphite/aluminum baffle that has a $13 \mathrm{~mm}$ beam aperture and is located immediately upstream of the target.

\subsubsection{Magnetic focusing horns}

Beyond the target are two, $3 \mathrm{~m}$-long, parabolic aluminum focusing horns. At each Main Injector beam spill, a $200 \mathrm{kA}$ current is pulsed over the inner and outer surfaces of the 


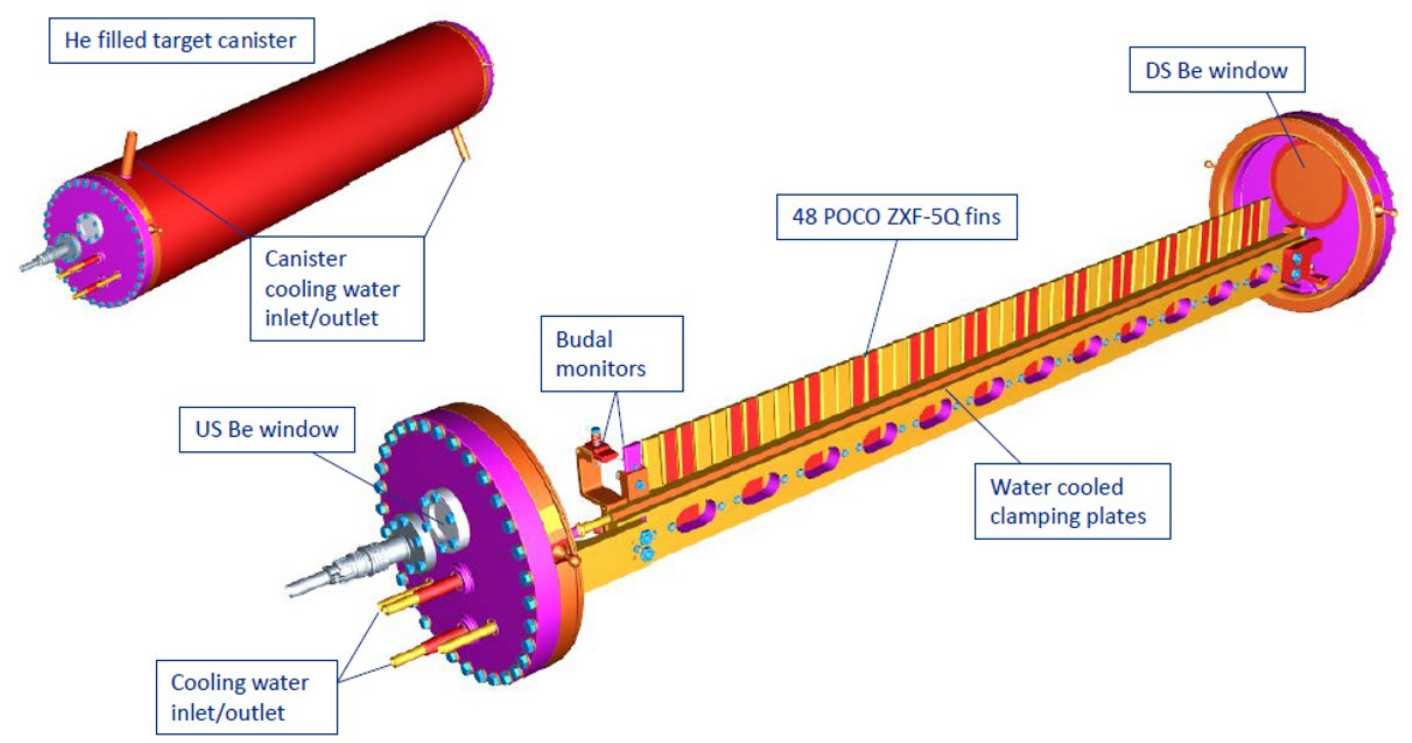

Figure 3.4: NuMI ME-era target. Figure from [47].

horns, parallel to the beam direction, in order to generate a torroidal magnetic field which deflects hadrons produced by the target. The horns must be sprayed with a $1.5 \mathrm{~mm}$ water layer to avoid overheating.
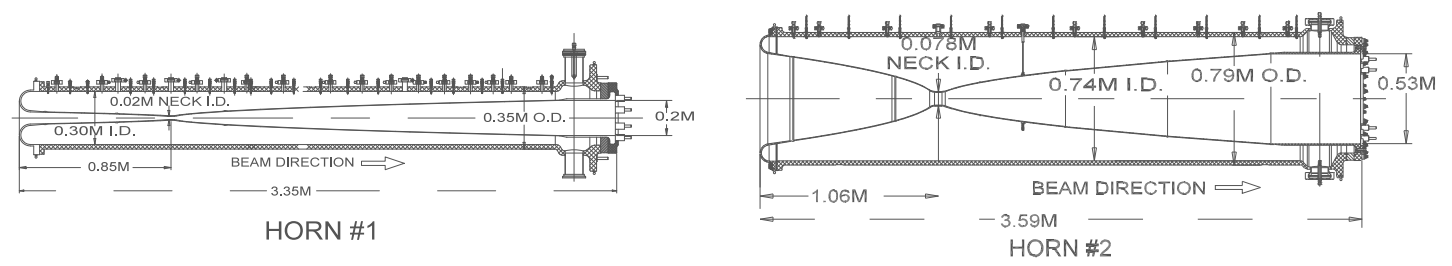

(a)

(b)

Figure 3.5: NuMI horns. Figures from [13].

In the forward horn current (FHC) configuration, also called "neutrino mode", the currents are aligned so that the magnetic field deflects negatively charged particles out of the 
beam and focuses positively charged particles such as $\pi^{+}$and $\mathrm{K}^{+}$, which decay into $\nu_{\mu}$ 's. The current polarity can be reversed (reverse horn current, RHC) to focus negative mesons and create an antineutrino-enriched beam.

Several parameters of the focusing system control the neutrino flux. The target-horn and horn-horn separation distances can be adjusted to focus different meson momenta. The horn current magnitude adjusts both the peak neutrino energy and the overall event rate.

Figure 3.6 shows possible hadron paths through the horns. In the Low Energy configuration, the target-horn 1 separation was minimized in order to focus the hadrons with the highest transverse momentum. For the ME, the target was removed from horn 1 to focus more forward hadrons resulting in the higher energy flux peak. By simultaneously moving horn 2 downstream forward mesons were focused more efficiently than by moving the target alone, resulting in the increased flux compared to the LE.

In the ME, the flux peak is composed predominantly of underfocused and overfocused neutrinos. Unfocused mesons, depicted by the solid red path in Figure 3.6, have low transverse momentum, typically decay to high-energy neutrinos and antineutrinos, and dominate the flux spectrum above the focusing peak.

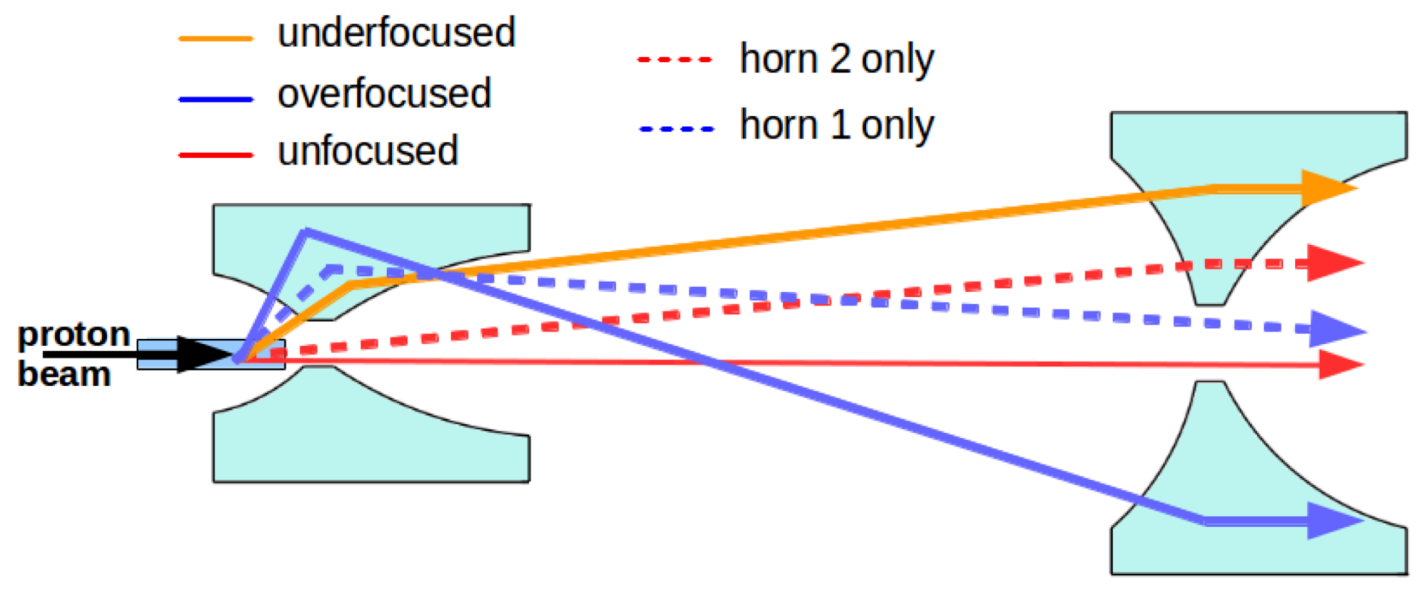

Figure 3.6: Hadron focusing components. Figure from [19].

Figure 3.7 shows the neutrino flavor breakdown of the NuMI beam. The vast majority of $\nu_{\mu}$ in the beam come from pion decays: $\pi^{+} \rightarrow \mu^{+} \nu_{\mu}$. Antineutrino, or wrong sign, 
contamination in the FHC beam often comes from unfocused, negatively charged mesons (typically $\pi^{-} \rightarrow \mu^{-} \bar{\nu}_{\mu}$ ) that did not pass through the horns' magenetic fields. Other $\bar{\nu}_{\mu}$, $\nu_{e}$, and $\bar{\nu}_{e}$ contamination comes from muon decays: $\pi^{+} \rightarrow \mu^{+} \nu_{\mu} \rightarrow \mathrm{e}^{+} \nu_{e} \bar{\nu}_{\mu} \nu_{\mu}$ and kaon decays: $\mathrm{K}^{+} \rightarrow \mathrm{e}^{+} \nu_{e} \pi^{0}$, as well as their charge conjugates.

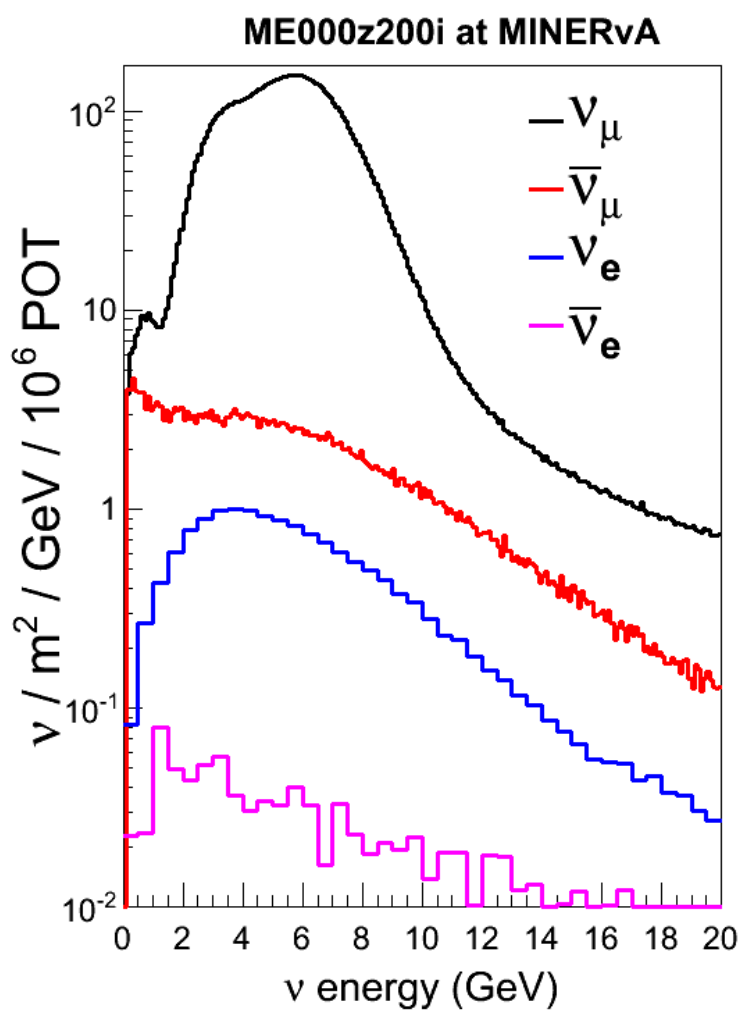

Figure 3.7: ME NuMI neutrino components. Figure from [19].

\subsubsection{Decay pipe and absorbers}

Downstream of the focusing system is a $675 \mathrm{~m}$-long, $1.8 \mathrm{~m}$ diameter helium-filled decay pipe, inside of which most of the pions and kaons decay into neutrinos. Beyond the decay pipe is a series of beam monitors and absorbers. Three muon monitors, each interspaced with dolomite rock, measure muon energy and can be used as an in situ proxy neutrino flux. In total, the beam passes through $240 \mathrm{~m}$ of dolomite rock before arriving at MINERvAenough rock to remove all muons from the beam. 


\subsection{SIMULATION AND FLUX PREDICTION}

A beamline simulation ultimately produces a flux prediction, which is a critical component to oscillation and cross section measurements alike. This section describes the NuMI beamline simulation and subsequent flux extraction.

The physics and geometry of NuMI are implemented in a sophisticated Monte Carlo simulation called G4NuMI. G4NuMI simulates the entire NuMI beamline, from initial Main Injector protons to the neutrino meson parent's point of decay. It is built on GEANT4 [15], which simulates the propagation of particles through matter, and it incorporates a detailed topological description of the beamline geometry.

The simulation output is in the dk2nu format [63] and includes full neutrino family trees including ancestor identities, kinematics, decays, paths through magnetic fields, and interactions with the target or surrounding beamline infrastructure. Ultimately, MINERvA's neutrino event generator GENIE uses the meson decay points and momenta to simulate neutrinos and their interactions in MINERvA's geometry. Post hoc constraints on the flux prediction from external data are implemented via event weights during the analysis stage, as described below.

In principle, the flux extracted should be well-defined from this simulation, but in practice, it can go wrong in two broad ways. The first is with respect to the focusing system. Dimensions, properties, and positions of the focusing components have inherent measurement uncertainties. Also, in implementing the simulation's geometric models, volumes and fields must sometimes be idealized and simplified. Current knowledge and uncertainties on the focusing system are discussed briefly in Section 7.4.2 and in more detail in Appendix A.

The second potential source of mismodeling of the NuMI prediction concerns so-called "hadron production", which refers to the entirety of particle interactions and reinteractions in the target and beamline aparatus. By default, hadron production is modeled by a combination of the FRITIOF precompound [99] and Bertini cascade [32][62] models (FTFP_BERT) in GEANT4. But meson reinteractions, which occur within a nucleus and are governed by nonperturbative QCD can be very complicated, and thus the GEANT4 model can differ significantly with data. 
For this reason, MINERvA uses external data to constrain the G4NuMI hadron production model. In particular the dominant portion of the flux, which comes from $\mathrm{pC} \rightarrow \pi^{+}$ interactions, are covered by measurements from NA49 [23]. To use the data, pion yields, $f_{\text {Data }}$, from NA49 inelastic interactions, given pion energy $E_{\pi}$, proton momentum $p$, and total inelastic cross section $\sigma_{\text {inel }}$,

$$
f_{\text {Data }}=\frac{1}{\sigma_{\text {inel }}} E_{\pi} \frac{d^{3} \sigma}{d p^{3}}
$$

are extracted from the NA49 data. After, each G4NuMI meson neutrino parent is weighted as a function of feynman $x_{F}$ and transverse momentum $p_{T}$,

$$
\operatorname{weight}\left(x_{F}, p_{T}, p\right)=\frac{f_{\text {Data }}\left(x_{F}, p_{T}, p_{0}=158 \mathrm{GeV} / c\right)}{f_{M C}\left(x_{F}, p_{T}, p_{0}=158 \mathrm{GeV} / c\right)} \times s\left(x_{F}, p_{T}, p\right)
$$

where $s$ is a scale factor from the NA49 proton beam momentum $158 \mathrm{GeV} / c$ to the Main Injector proton beam momentum $120 \mathrm{GeV} / c$, calculated by an independent physics list, FLUKA [48]. Figure 3.8 shows the phase space covered by NA49 and its application to the MINERvA flux prediction. Note that G4NuMI overpredicts $\pi^{+}$production by $\sim 20 \%$ near the MINERvA neutrino production peak at $\left(x_{F}, p_{T}\right)=(0.5,0.2 \mathrm{GeV} / c)$.

Other constrained processes, covered phase spaces, and datasets considered are described in Section 7.4.1 and in great detail in [19] and [21]. The ME flux, constrained by all hadron production data, is shown in figure 3.9. The error band uses LE-era focusing uncertainties, but otherwise the final ME flux is shown. 


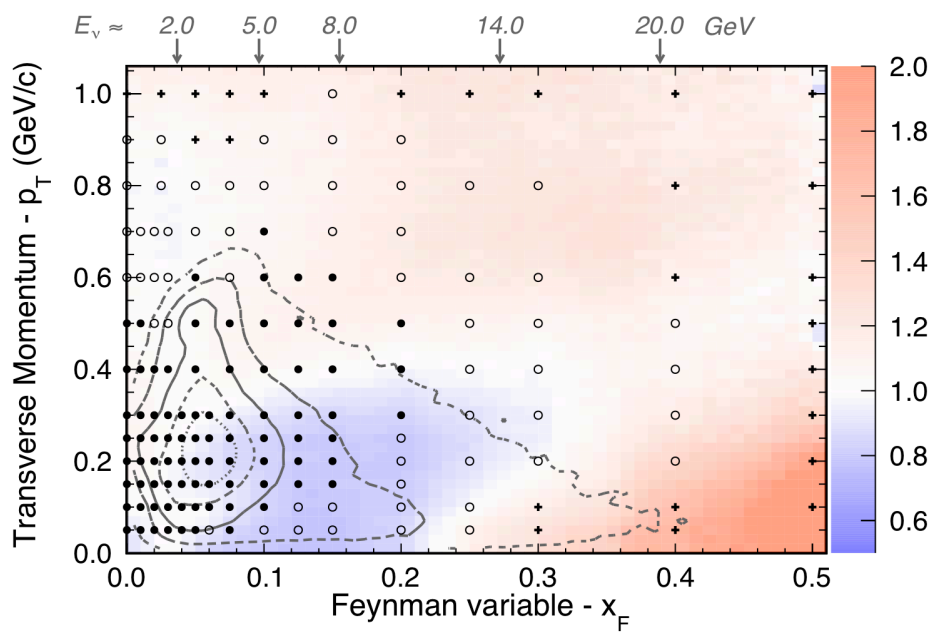

Figure 3.8: Pion phase space covered by NA49 data. Markers show the location of NA49's measurements of the invariant cross section for $\mathrm{pC} \rightarrow \pi+\mathrm{X}$ interactions as a function of the pion's $x_{F}$ and $p_{T}$. Marker types correspond to statistical uncertainties, the color scale shows the size of the correction to the default G4NuMI model, and the central contour show the peak phase space of $\mathrm{pC} \rightarrow \pi+\mathrm{X}$ interactions leading to $\nu_{\mu}$ in MINERvA for the LE beam. Figure from [21]. 


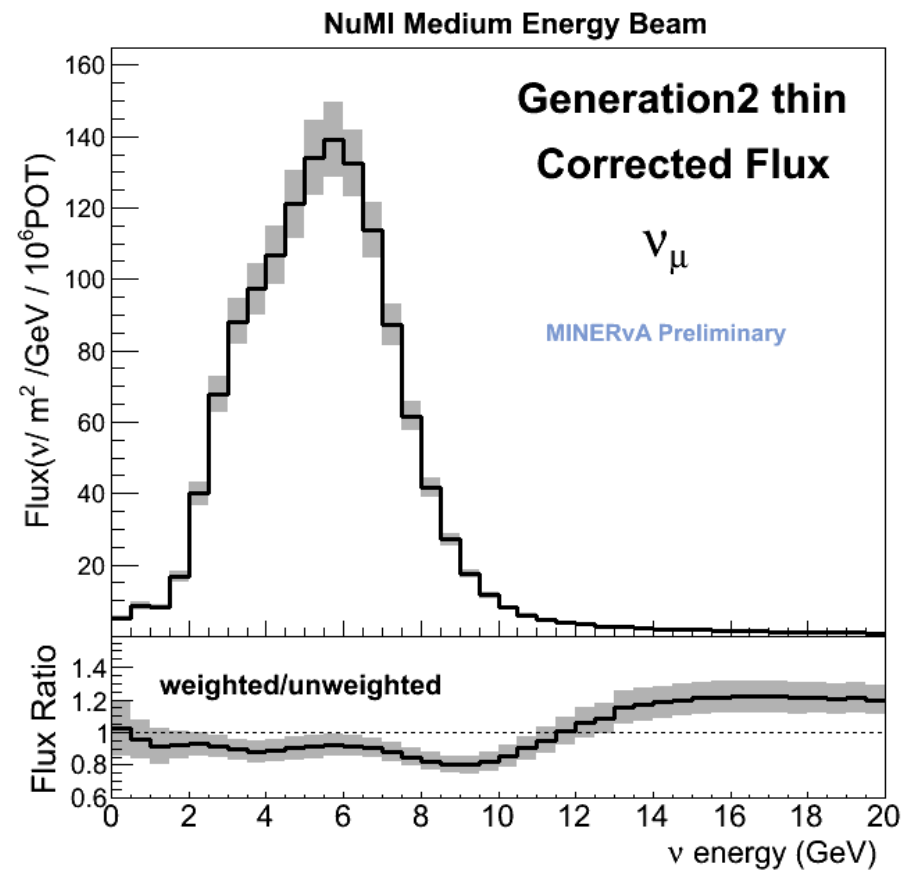

Figure 3.9: ME, hadron production constrained ME flux. The error band uses LE-era focusing uncertainties. Figure from [19]. 


\subsection{MINERVA NEUTRINO DETECTOR}

The MINERvA experiment was conceived in the early 2000's [1] as a collaboration of nuclear and particle physicists with the primary goal of measuring neutrino interaction cross sections on a variety of heavy nuclei, in order to serve the coming decades of neutrino oscillation experiments by improving the interaction models that are critical to those measurements. MINERvA completed construction in 2013, and took data until February 2019. In total 16.1e20 neutrino-mode protons on target (POT) and 14.1e20 antineutrino-mode POT were collected at two NuMI beam energies, $\left\langle E_{\nu}\right\rangle \sim 3.5 \mathrm{GeV}(\mathrm{LE})$ and $\left\langle E_{\nu}\right\rangle \sim 6 \mathrm{GeV}(\mathrm{ME})$. Many important measurements have still to be made, and MINERvA is currently entering a "data preservation" era.

This chapter first discusses the detector design and method of neutrino interaction detection (Section 4.1). Next, detector calibrations are discussed - the process which converts raw data into hits of certain energy (Section 4.2). After calibrations, the reconstruction of hits into tracks and physics events is explained (Section 4.3). And finally, there is a presentation of the sophisticated detector simulation which models neutrino interactions through the detector, including full electronic response, readout, and calibration (Section 4.4).

\subsection{DESIGN}

The MINERvA detector, shown in Figure 4.1 is a $5 \mathrm{~m}$-long, $1.7 \mathrm{~m}$-apothem regular hexagonal prism aligned along a z-axis parallel to the detector hall floor. The NuMI beam, which is directed through the earth towards the MINOS far detector in Minnesota, points $3.3^{\circ}$ downward relative to the detector z-axis. 
The detector has a central, $\sim 2.5$ m-long, $85 \mathrm{~cm}$ apothem fully active tracking core, which is surrounded by a partially active lead and steel shell that provides support, containment, and some tracking. Downstream of the central tracking region, at the end of the detector, are partially active electronic and hadronic containment calorimeters (ECAL and HCAL). Upstream of the tracking region are several partially active nuclear targets of various materials used to study the atomic mass scaling of neutrino interactions.

The detector is composed of 208 hexagonal planes stacked along the z-axis. Tracking planes are composed of interleaved triangular prism strips (pixels) of plastic scintillator. In the downstream calorimeter region, passive planes of lead (ECAL) and steel (HCAL) are alternated with tracking planes to provide good containment while also maintaining fine position resolution. In the nuclear target region, passive nuclear target planes are interspersed with tracking planes. Altogether, MINERvA contains more than 30,000 active pixels, and tracks are reconstructed from a particle's energy deposits in consecutive planes and pixels.

The design of each of these aspects - the active tracking planes, subdetector regions, and adjacent MINOS near detector — are described in the following sections. After, an explanation of the detection process - light detection, signal readout, and electronics — is discussed.

\subsubsection{Active tracking planes}

Each of the 208 active tracking planes is 1.7 cm-thick and composed of 127 hydrocarbon strips glued together with translucent epoxy.

Planes are stacked in one of three orientations (Figure 4.2), each rotated along the z-axis with respect to the other. The strips of an X-plane are aligned vertically, providing horizontal hit information. The U- and V-planes are aligned $60^{\circ}$ clockwise and counterclockwise with respect to the X-plane. Planes are stacked in groups of XUXV. While two orientations can provide 3D particle tracking, three such orientations removes ambiguities that can occur when two particles deposit hits in orthogonal planes.

Each strip is a triangular prism with $17 \mathrm{~cm}$ height and $3.3 \mathrm{~cm}$ base. Interleaved triangular strips ensure that particles deposit energy in two adjacent strips when passing through a 


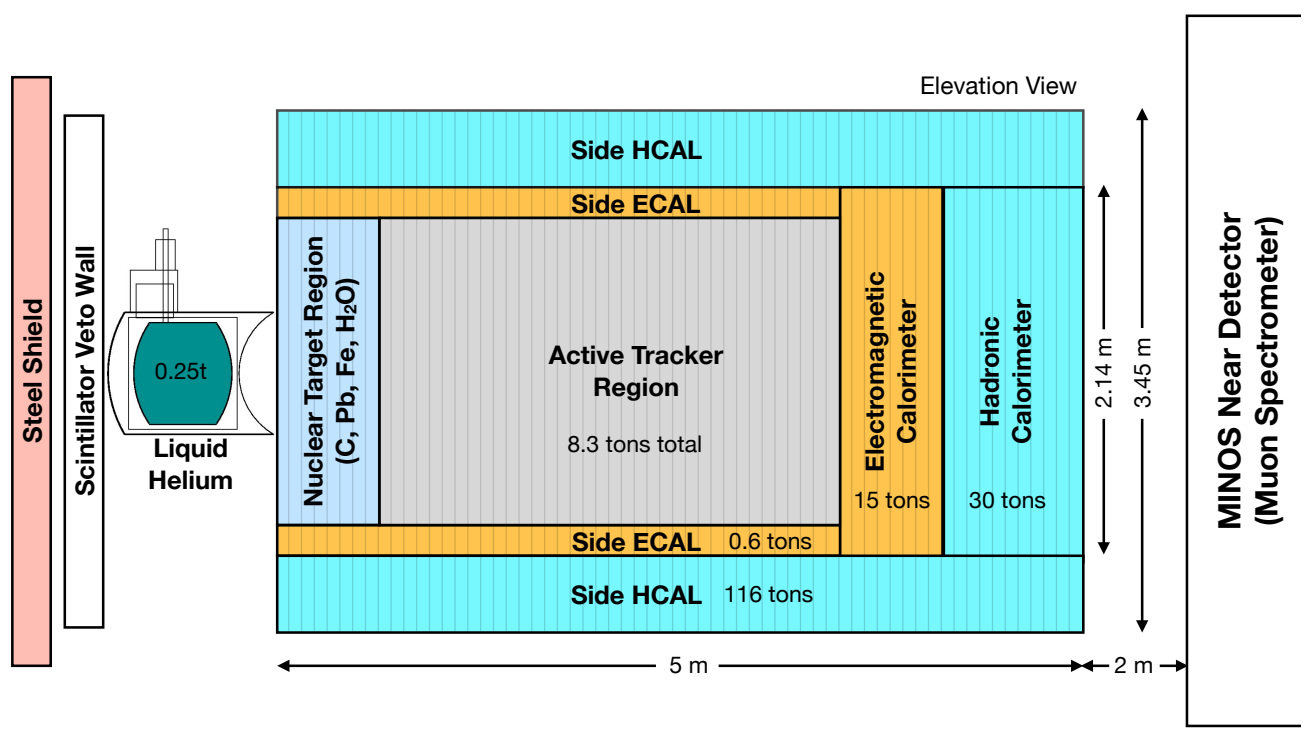

(a) MINERvA detector side view. The beam enters from the left.

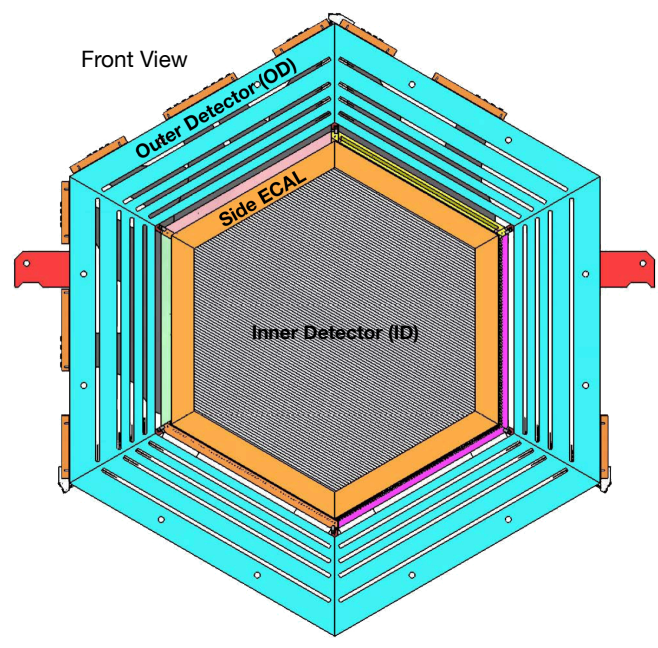

(b) MINERvA tracking plane front view.

Figure 4.1: MINERvA detector side view and tracking plane front view.

plane, thereby reducing the position resolution compared to similarly-sized rectangular strips (Figure 4.3). A position resolution of $3 \mathrm{~mm}$ is obtained by using the relative size of energy deposits in the two strips. Furthermore, the triangular strip geometry allows planes to be 

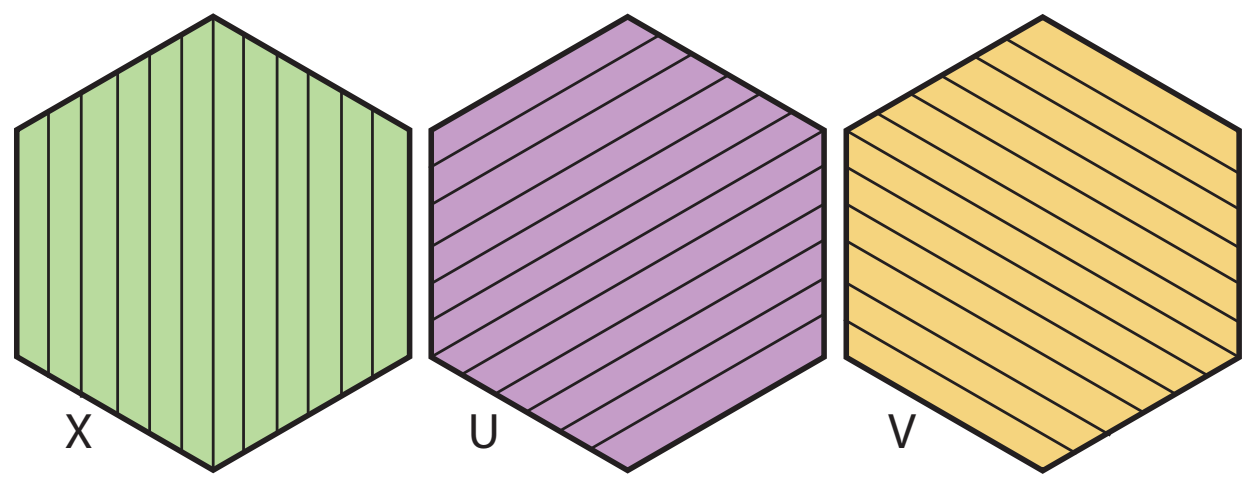

Figure 4.2: Diagram representing the three $\mathrm{X}, \mathrm{U}$, and $\mathrm{V}$ plane orientations.

precisely calibrated in situ by leveraging the fact that minimum ionizing particles deposit the most energy in a strip when they pass directly through its center.
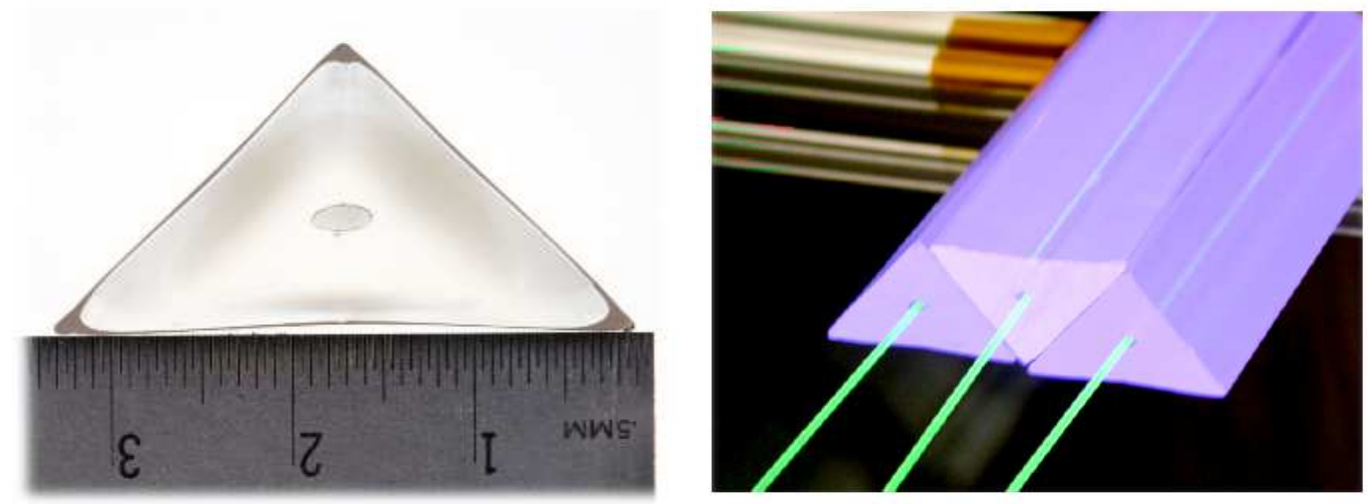

Figure 4.3: Scintillator strip triangular cross section, and interleaved plane geometry. Figure from [20].

Strips are polystyrene and doped with two wavelength-shifting organic scintillators. The strips are co-extruded with a $0.25 \mathrm{~mm}$ reflective coating of polystyrene and titanium dioxide. A $2.6 \mathrm{~mm}$-diameter hole is bored through the length of each strip $8.5 \mathrm{~mm}$ above the triangle base, and a wavelength-shifting readout optical fiber is fed through the hole. 


\subsubsection{Subdetector regions}

The central tracking region spans 127 planes, and, as it is fully active, is used as a large continuous volume to detector neutrino interactions. Immediately downstream is the ECAL, which consists of 20 tracking planes interspersed with a $0.2 \mathrm{~cm}$-thick lead plate $(0.35$ electromagnetic interaction lengths) placed before every second active plane. Beyond the ECAL is the HCAL, consisting of 20 active planes interspersed with a $2.54 \mathrm{~cm}$-thick steel plate (3. nuclear interaction lengths) placed between every active plane. The ECAL and the HCAL contain electromagnetic and hadronic showers in order to improve recoil and neutrino energy reconstruction.

Upstream of the tracking region is the nuclear target region. This region is composed of 44 active planes, interspersed with five, similarly-shaped, passive planes of solid materials such as lead, iron, carbon. Additionally there is a hexagonal water tank target among the planes, as well as a 2,300 liter liquid helium tank at the furthest upstream end of the nuclear target region. The nuclear target region is illustrated in Figure 4.4. Data from this region is not used in this analysis, but interactions on nuclear targets can be used to make cross section ratios between materials, in order to better understand $A$-scaling of neutrino interactions. At the furthest front end of the detector is a veto wall consisting of layered steel-scintillator planes. The wall tags muons created from neutrino interactions upstream of MINERvA in the rock of the detector wall.

Tracking planes are surrounded by a $10 \mathrm{~cm}$ lead electromagnetic containment collar and an outer steel frame with eight scintillator strips. The frame provides structural support to the planes, outer hadron calorimetery, and side-exiting muon identification.

\subsubsection{MINOS near detector}

Located $2 \mathrm{~m}$ downstream of MINERvA is the MINOS (Main Injector Neutrino Oscillation Search) near detector [90]. MINOS is a one kiloton magnetized scintillator detector and iron calorimeter. For MINERvA's purposes, it is used to measure the charge and momentum of muons that exit the back of the detector. Figure 4.5 shows the path of a muon exiting the back of MINERvA and entering MINOS, and Figure 4.6 shows the front face of the 


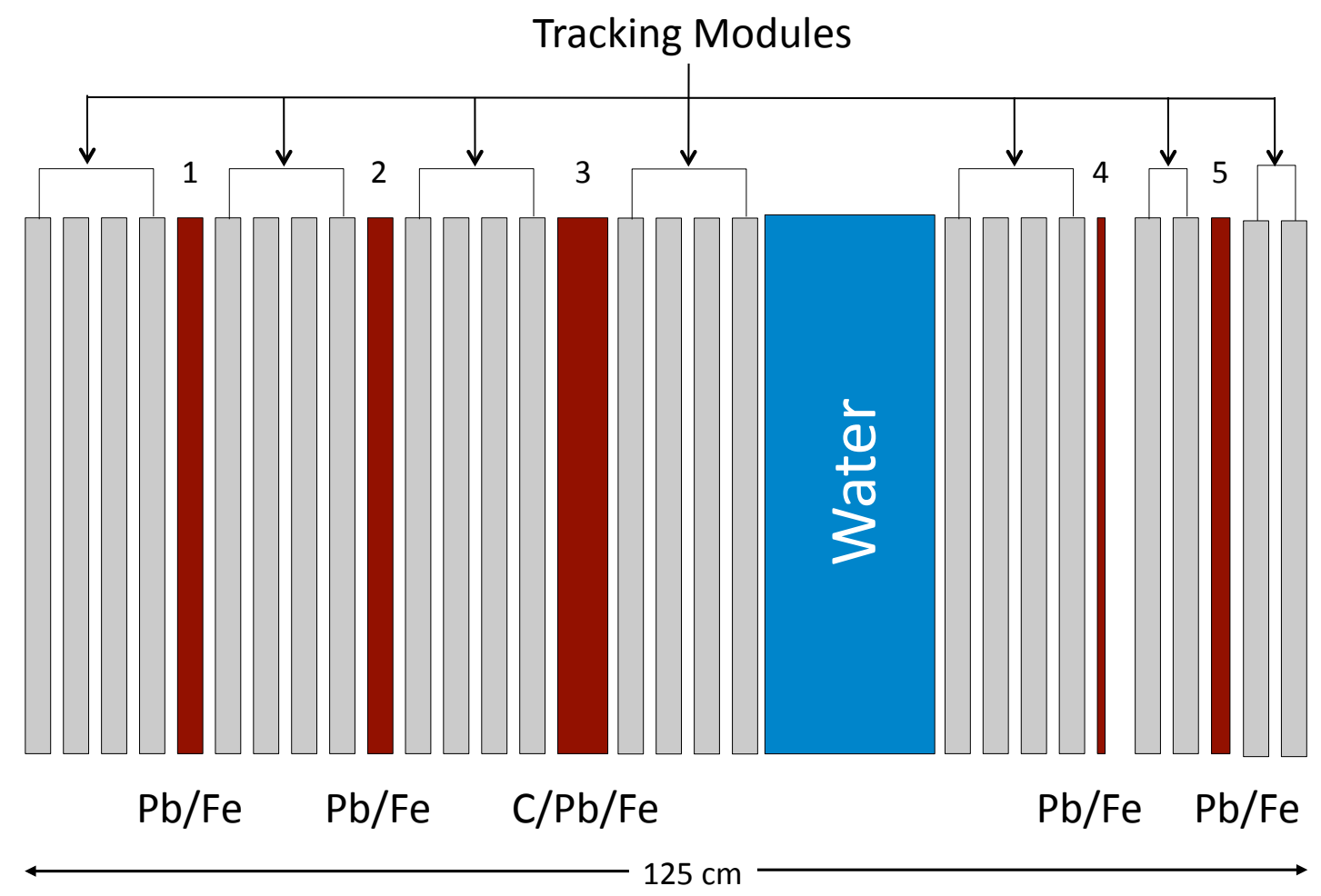

Figure 4.4: MINERvA nuclear target region composition. Liquid helium cryostat not pictured. Figure from [20].

MINOS detector, including the NuMI beam centroid and the location of the magnetic coil. Muon electric charge sign is determined from its direction of curvature in the 1.3-T average magnetic field, and it is used as the primary indicator of whether a given interaction was due to an incident neutrino $\left(\mu^{-}\right)$or antineutrino $\left(\mu^{+}\right)$. Muon momentum can be measured to within $2 \%$ by track range or curvature. Momentum is used to calculate the lepton side of the neutrino interaction process. Muons greater than $\sim 10 \mathrm{GeV}$ are not contained in MINOS and must be measured by curvature. Depending on the NuMI horn current polarity, the polarity of the magnetic field is aligned to focus $\mu^{-}$(during FHC) and $\mu^{+}$(during RHC) toward the coil to maximize muon track length and maximize the muons of the neutrinos of interest. Muon reconstruction is discussed in Section 4.3.4. Figure 4.7 shows the MINOSMINERvA-NuMI relative positions and MINERvA coordinate sytem. 


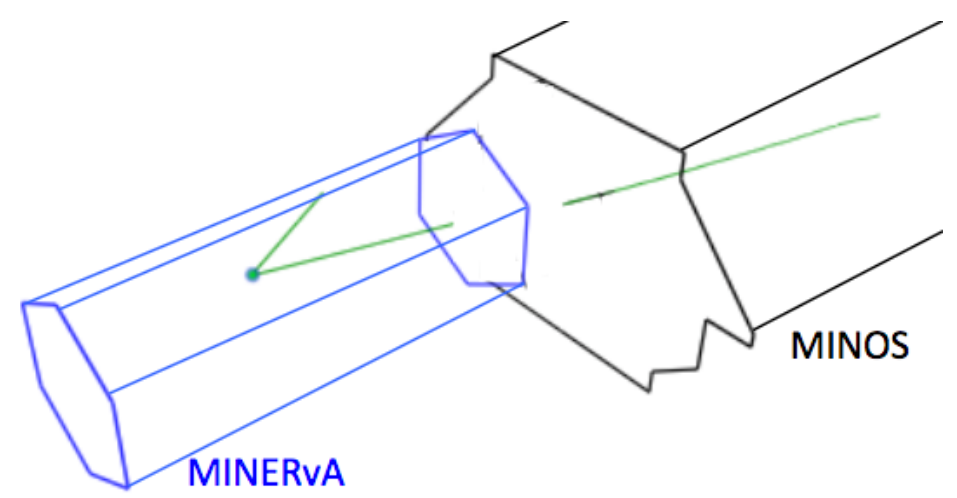

Figure 4.5: Muon track exiting MINERvA and entering MINOS.

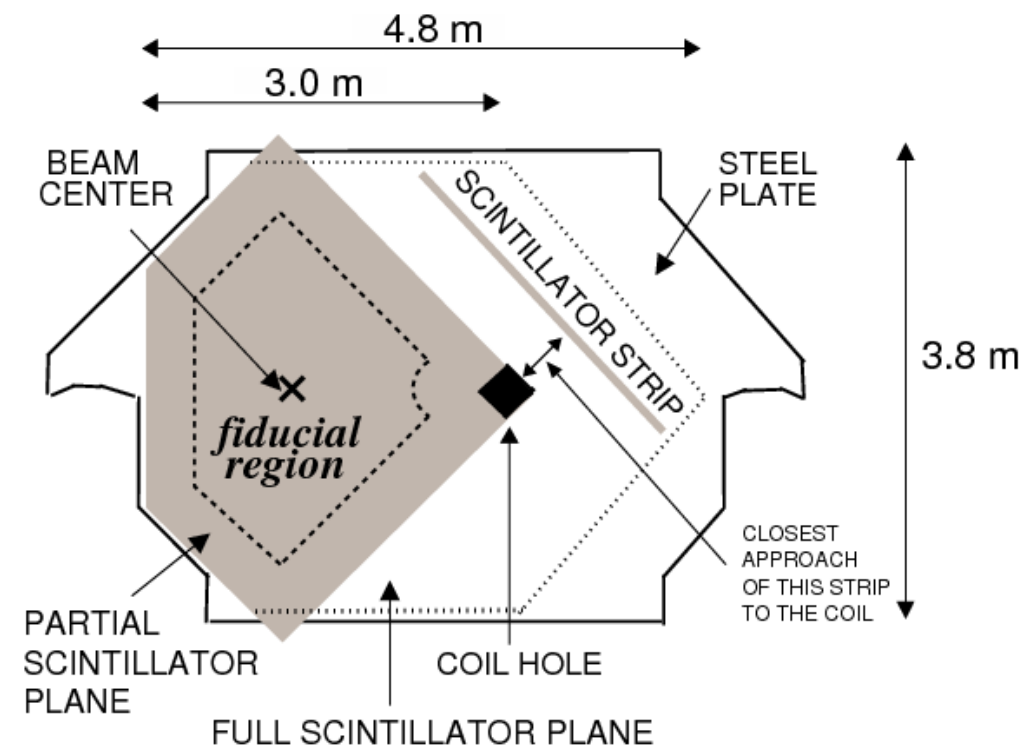

Figure 4.6: MINOS near detector front face.

\subsubsection{Light detection, readout, and electronics}

When a charged particle passes through a MINERvA plane, a strip's blue-emitting scintillator is read out by a $1.2 \mathrm{~mm}$ diameter green wavelength shifting (WLS) optical fiber set in the center of the strip. One fiber end is mirrored and the other is grouped with the fibers of other strips, and transmitted by a sequence of connectors to a Hamamatsu 64-anode 


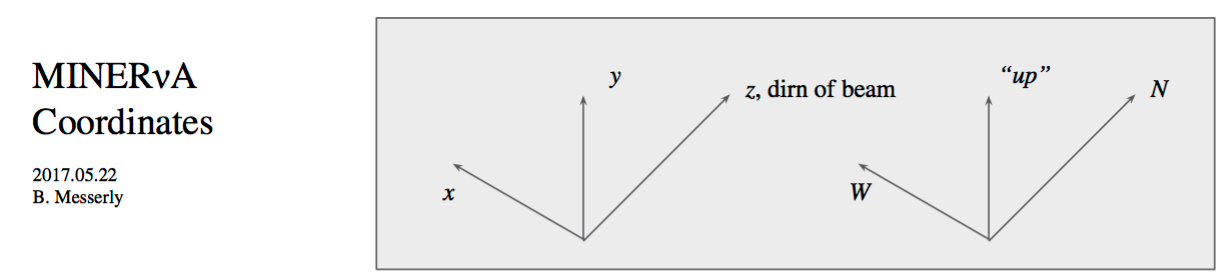

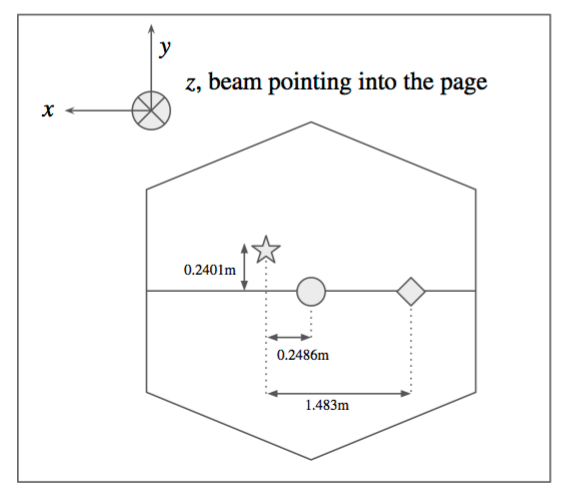

Looking down the beamline

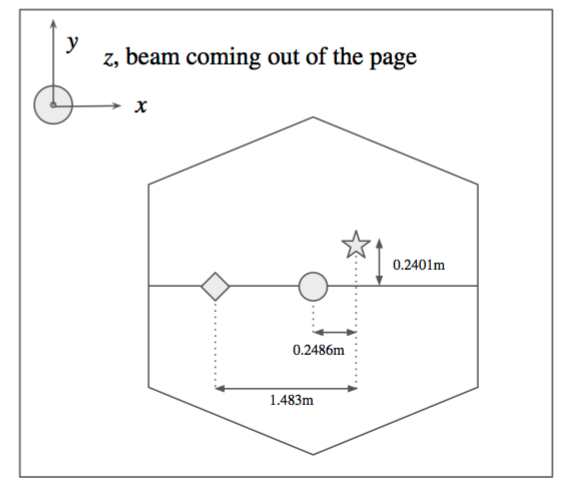

Looking up the beamline (normal plotting)

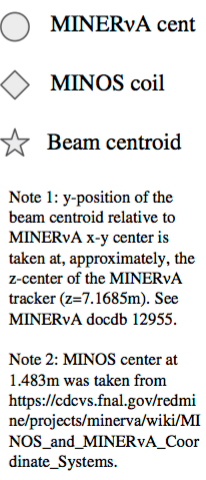
NOS_and_MINERvA_Coor
dinate_Systems.

Figure 4.7: Shows the MINERvA orientation relative to the MINOS magnet coil and NuMI beam centroid. Recall, the beam points $3.3^{\circ}$ downwards with respect to MINERvA z-axis. Figures are shown at approximately the longitudinal center of the MINERvA detector.

photomultiplier tube to amplify the signal.

Optical fibers from 64 strips are arranged in an $8 \times 8$ grid measuring $4 \mathrm{~cm}^{2}$ on the PMT. Each fiber connects to an individual, 12-stage dynode amplification chain. A typical dynode chain gain is 5 e5 output electrons to each input photoelectron arriving at the first dynode. The scintillation light of a typical minimum ionizing particle produces 1-10 photoelectrons, which results in a 200-500 $\mathrm{fC}$ current at the anode.

The signal from the PMT is read out by a Front End Board (FEB). Six ApplicationSpecific Integrated Circuit (ASiC) chips (TriP-t chips) digitize and store charge with ADCs. A group of three TriP-t's each service 32 of the PMT anodes. Among each group of three there is a TriP-t with a high, medium, and low gain, which simultaneously digitize the signal.

FEBs are connected into chains of 3-11 boards, which are read out by a Chain ReadOut Controller (CROC). CROCs then receive timing and trigger information from a custom 


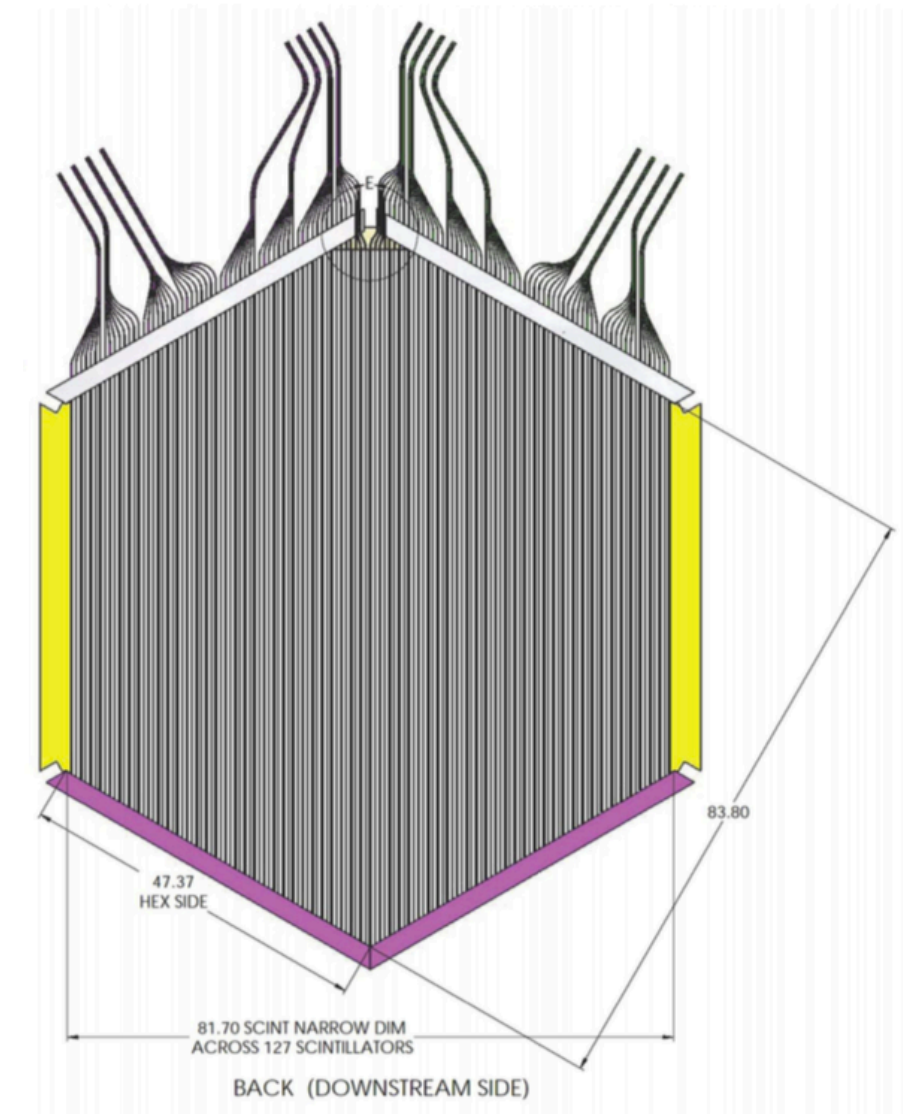

Figure 4.8: Engineering drawing of a tracking plane showing the WLS readout fibers. Figure from [1].

CROC Interface Module (CRIM). A trigger is activated from a NuMI beam spill signal and a readout gate is activated for $16 \mu \mathrm{s}$ - starting $500 \mathrm{~ns}$ before the spill arrives, and continuing to readout for $5.5 \mu$ s after the spill ends.

The energy deposition information in a strip (referred to also in the context of data readout as a pixel or channel) is organized into hits. A hit in a strip due to a particle energy deposit is defined by a timestamp relative to the gate start. A single strip can have several hits readout within a single gate. A hit is formed when the amount of charge from the PMT exceeds a certain discriminator threshold. At that point a hit timestamp is created and charge is integrated for the following 151 ns. The integrated charge is associated with the 


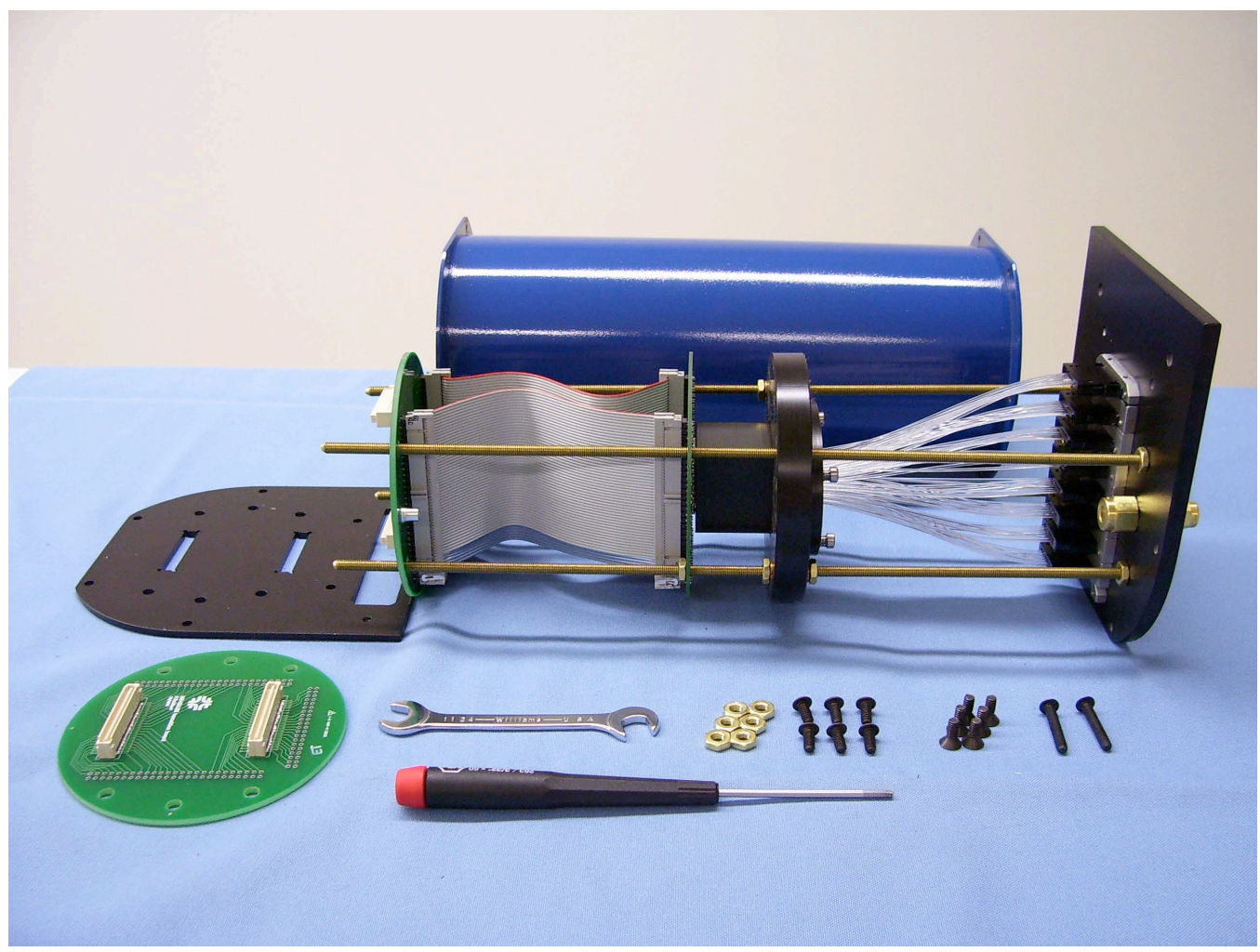

Figure 4.9: Optical tube before construction. The photomultiplier is the black box. Figure from $[20]$.

hit, and after the end of the gate, all hits are readout from the FEB's to nearline storage. Hit timestamps have a resolution of 2.35 ns from FEB precision.

Following the 151 ns charge integration time, 188 ns are required to reset. During this dead time, charge can not be collected, and hits may be missing from spatially and temporally coincident neutrino interactions.

\subsection{CALIBRATION}

A series of calibrations procedures converts raw ADC counts (the collection of which was described in the previous section) into hit energy, accounting for the various components 
described in the previous section and pictured in Figure 4.10. Hit timing, response differences between pixels, as well as variations in individual pixel responses over time are accounted for.

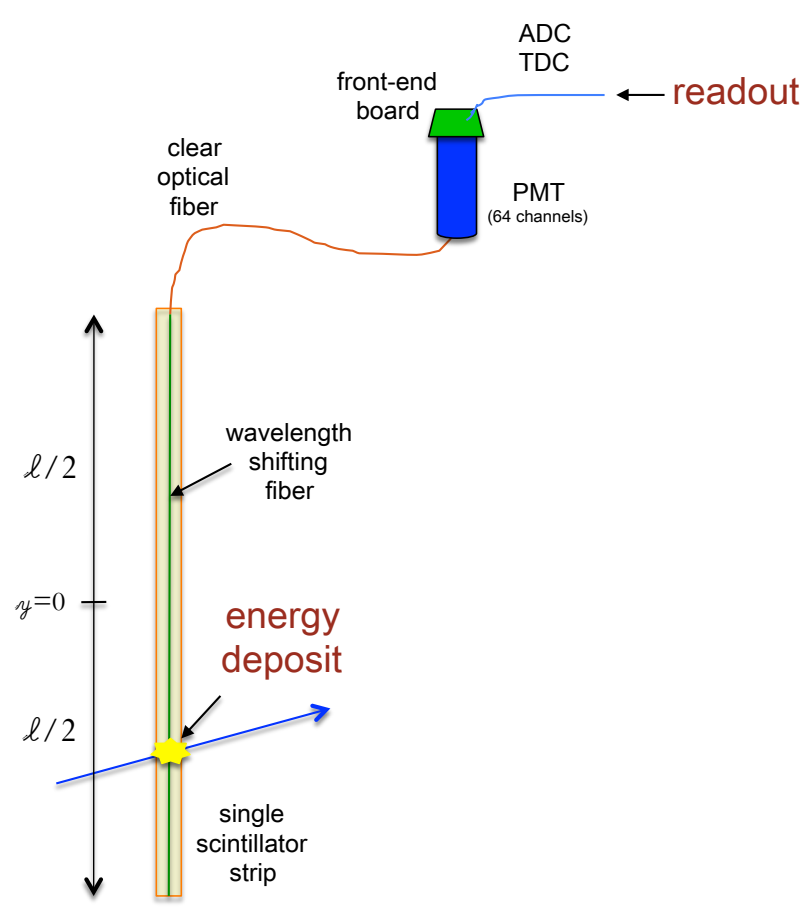

Figure 4.10: Schematic of a single optical readout channel. The calibrations process converts raw ADC counts into energy. Figure from [20].

The full calibration procedure taking the ADC counts of strip $s$ to its energy $E_{s}$ is described by

$$
E_{s}(t)=\left(\mathrm{ADC}_{s}(t)-\mathrm{Ped}_{s}(t)\right) \times \mathrm{FEB}_{s} \times \frac{1}{\operatorname{Gain}_{s}(t)} \times \operatorname{Atten}_{s} \times S_{s}(t) \times C(t)
$$

where Ped is the detector noise pedestal, FEB characterizes a front end board's analogto-digital gain, Gain is a PMT's gain, Atten accounts for both the attenuation of energy within a strip as well as the within the optical transmission fibers, $S$ is the "strip-to-strip" correction accounting for variations across channels, and $C$ is an overall energy scale for the whole detector. The ex situ corrections independent of time were measured external 
to MINERvA construction and in situ corrections dependent on time are measured during regular data taking and can be valid for days or weeks depending on the procedure.

The procedure can be divided into three conceptual steps: conversion from raw hit ADC counts to PMT photoelectrons $\left(\left(\mathrm{ADC}_{s}(t)-\operatorname{Ped}_{s}(t)\right) \times \mathrm{FEB}_{s} \times \frac{1}{\operatorname{Gain}_{s}(t)}\right)$, conversion from PE to hit energy $\left(\operatorname{Atten}_{s} \times S_{s}(t) \times C(t)\right)$, and time calibration.

\subsubsection{ADC to PE calibration}

The ADC to PE calibration itself consists of three steps, which are discussed in the following sections. First detector noise (pedestal) is subtracted. Next digital counts are converted into a charge. And finally the PMT gain converts the charge into a number of photoelectrons arriving at the PMT's first dynode.

\subsubsection{Pedestal subtraction and suppression A pedestal is a measure of back-} ground detector noise when no beam activity is expected in the detector. The pedestal is measured once per day inbetween the regular data collection of NuMI beam spills. Figure 4.11 shows a pedestal histogram in terms of ADC counts for a single channel, collected over the course of many $16 \mu$ s gates.

Outlying gates, such as the one shown in 4.11 can arise due to electronics noise, cosmic ray muons or radioactivity. They are removed from the pedestal measurement using Peirce's Criterion [114] and the mean and RMS is calculated from the resulting distribution. To apply the pedestal subtraction to data, first the pedestal mean is subtracted from the pixel's raw ADC counts, and second, the subtracted hits are removed (suppressed) if they do not satisfy a certain hit threshold. Pedestals are found to vary by $\sim 7 \%$ across pixels and by $2 \%$ within a single gate, and they are found to be stable over time.

4.2.1.2 FEB ADC-to-charge conversion After pedestal subtraction, the ADC counts of a hit are converted into charge by looking up a analog-to-digital response constants in a database. Before MINERvA's construction, the response constants of more than 500 front end boards were measured by a dedicated test stand. By providing a known amount of input 


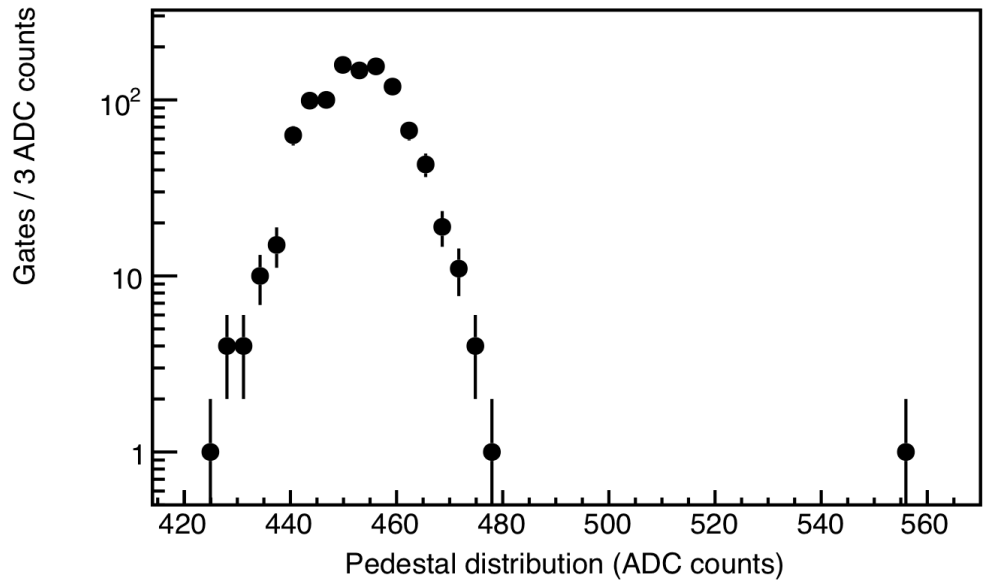

Figure 4.11: Example of a channel's pedestal measurement, including an outlier gate. Such gates are removed before calculating pedestal means and widths. Figure from [20].

charge, the digital response of the six TriP-t integrated circuits mounted on each board was measured. The response was best characterized by a tri-linear fit, consisting of three distinct linear segments. In total, 18 constants, corresponding to the slope and intercept for each segment, and each low, medium and high gain response, are stored in a database for each strip. Figure 4.12 shows the low, medium, and high trilinear fits for a sample channel. Note each gain saturates around 2500-3000 ADC counts.

4.2.1.3 Photomultiplier tube gain After conversion from ADC counts to charge, a PMT's production of charge per incoming photoelectron, i.e. its gain, is accounted for:

$$
g=\frac{\text { output charge }}{\text { input photoelectrons }}=\frac{Q}{\lambda e}
$$

Once per day, in between NuMI beam spills, light from a flashing LED is injected into each PMT and the output is measured for each strip. The light injection system injects a constant but variable amount of light (typically 1-10 PEs), and the output ADC spectrum is fit to a theoretical function that has been built from assumptions about the PMT's internal 


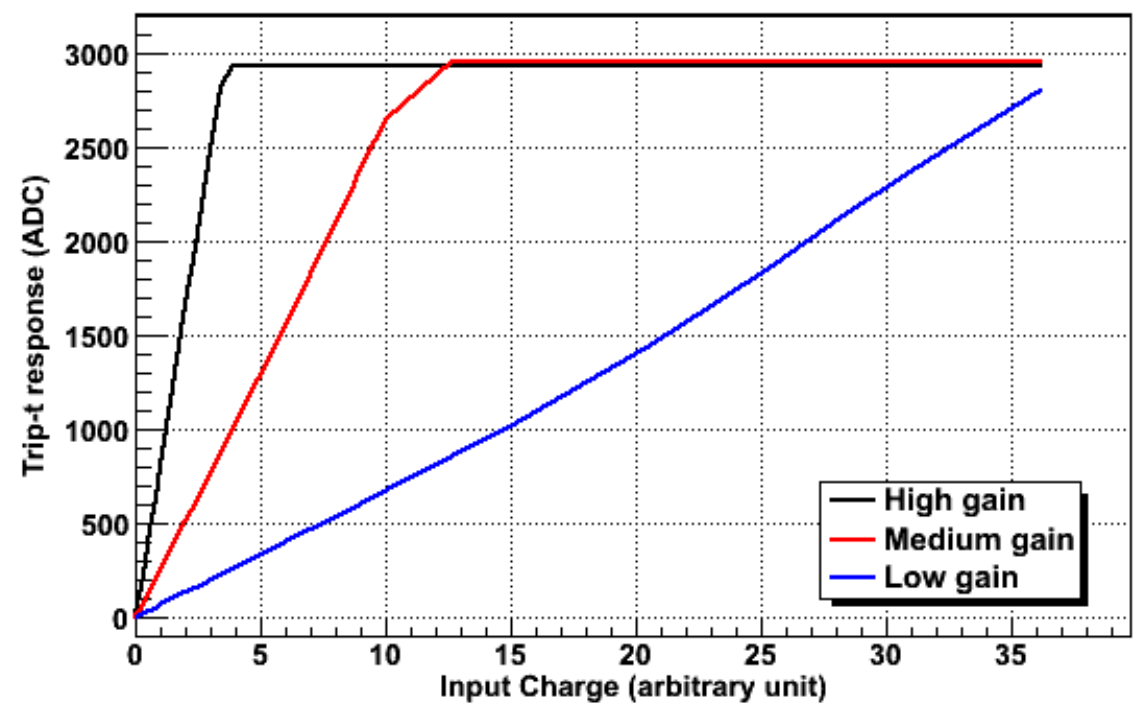

Figure 4.12: Low, medium, and high gain response of one strip and a typical front end board. Figure from [20].

operation. In particular, the probability distribution of measuring an output charge $q$ given a number of input PEs $\lambda$ is composed of (i) a Poisson distribution for the first dynode's amplification of the initial PEs, (ii) another Poisson for the subsequent dynode amplification, and (iii) a Gaussian representing the spread of pedestal noise [103]. Then the measured variance of this distribution can be written as a function of and subsequently solved for the gain value [85][20]. Channel gains are stable to less than $3 \%$, and have been found to be increasing on average at a rate of $\sim 0.02 \%$ per day.

\subsubsection{PE to energy calibration}

To convert photoelectrons into hits of energy with proper $\mathrm{x}-\mathrm{y}-\mathrm{z}$ position, first energy loss due to optical fiber attenuation is accounted for. Then plane misalignments are corrected. After, the energy response across strips is corrected, and finally, a detector-wide absolute energy scale is set. 


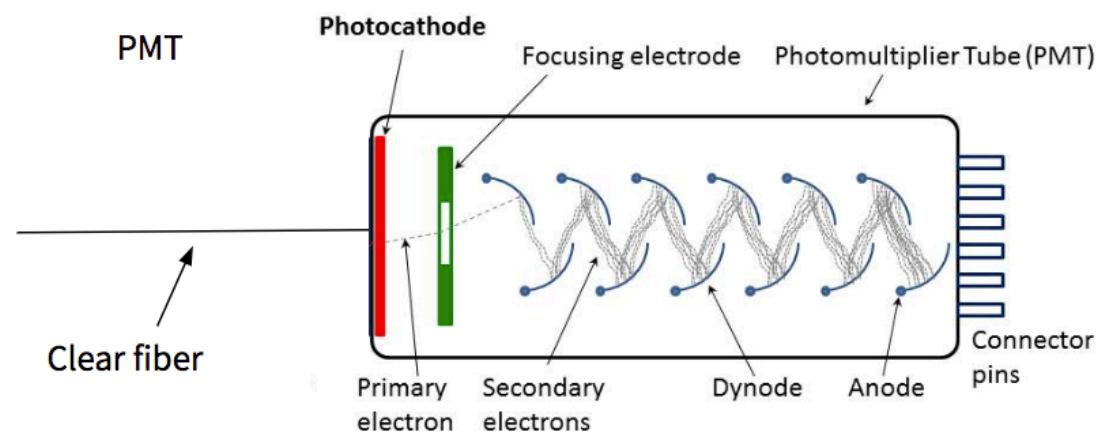

(a) Illustration of a PMT's 12-dynode gain avalanche.

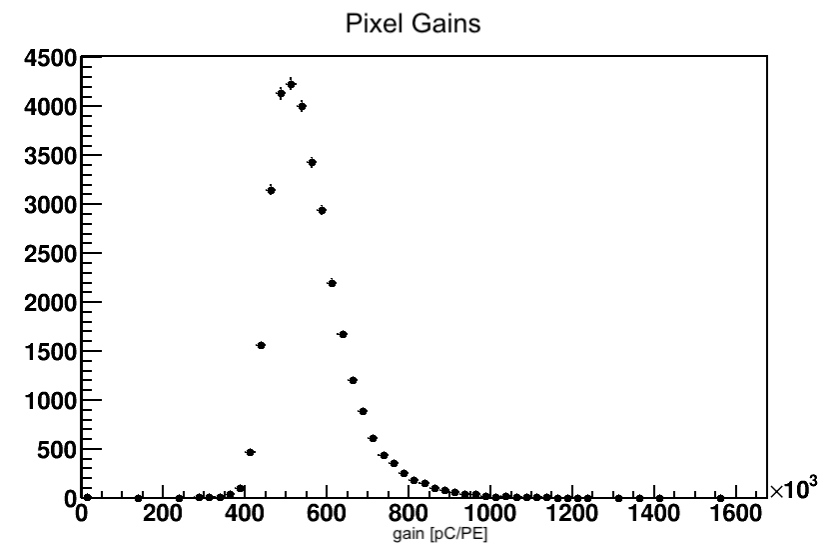

(b) Typical gain distribution of all $\sim 32,000$

MINERvA channels.

Figure 4.13: PMT operation diagram and typical gains distribution of all MINERvA channels.

4.2.2.1 Optical fiber attenuation The output of the PMT gain calibration is a number of PEs that reach the PMT. The next calibration factor accounts for light attenuation between the hit location and PMT. Separate attenuation constants are used for the WLS fibers within the scintillator strips and for two clear fiber connecting sections between a strip edge and PMT. The latter two constants are fixed for a given strip, because the light travel 
distance is constant. The WLS attenuation correction depends on the hit position along the strip, provided a three-dimensional object can be formed with surrounding strips and planes. In absence of a three-dimensional object, the travel distance is assumed to be half the length of the strip. Attenuation constants were originally measured before MINERvA construction by scanning a radioactive Cs-137 source along the length of each strip.

4.2.2.2 Plane alignment Subsequent calibration steps require knowledge of track path lengths which in turn requires that the position of each plane be known to better than 1 $\mathrm{mm}$ in the $\mathrm{x}-\mathrm{y}$ plane. Plane position is determined by leveraging the fact that normally incident particle tracks deposit maximum energy when passing through the middle of the strip. Using a sample of through-going rock muons (typically created by neutrino interactions in the rock downstream of the NuMI decay pipe), the average energy as a function of strip triangle base position is fitted to the shape of the strip as illustrated in Figure 4.14. The peak of the fit becomes a translational shift parameter for the plane, which is treated as a rigid structure. From this method a plane rotation about the z-axis can be discerned. The alignment correction is very stable and precise to within $0.3 \mathrm{~mm}$ and $0.5 \mathrm{mrad}$ on average. The rock muon sample of at least 300,000 muons is collected over the course of weeks or months using in-spill data.

4.2.2.3 Relative strip-to-strip response variations The peak energy loss per unit path length for a muon of $\sim 1 \mathrm{GeV}$ is largely independent of the precise energy of the muon. This fact, together with the rock muon sample described in 4.2.2.2 is used to standardize the response of each plane, which can vary due to any number of manufactoring or detector construction inconsistencies.

The strip correction requires the truncated mean energy per unit path length deposited by rock muons to be the same in every strip. The truncated mean is computed iteratively and has the advantage of only requiring hundreds of muons passing through a strip, rather than thousands required by a fit. In the first iteration of the truncated mean calculation, the mean is taken to be the full mean of events from 0 to $20 \mathrm{MeV} / \mathrm{cm}$. In subsequent iterations, the mean considers only energies per path length within $\pm 50 \%$ of the previous mean. The 

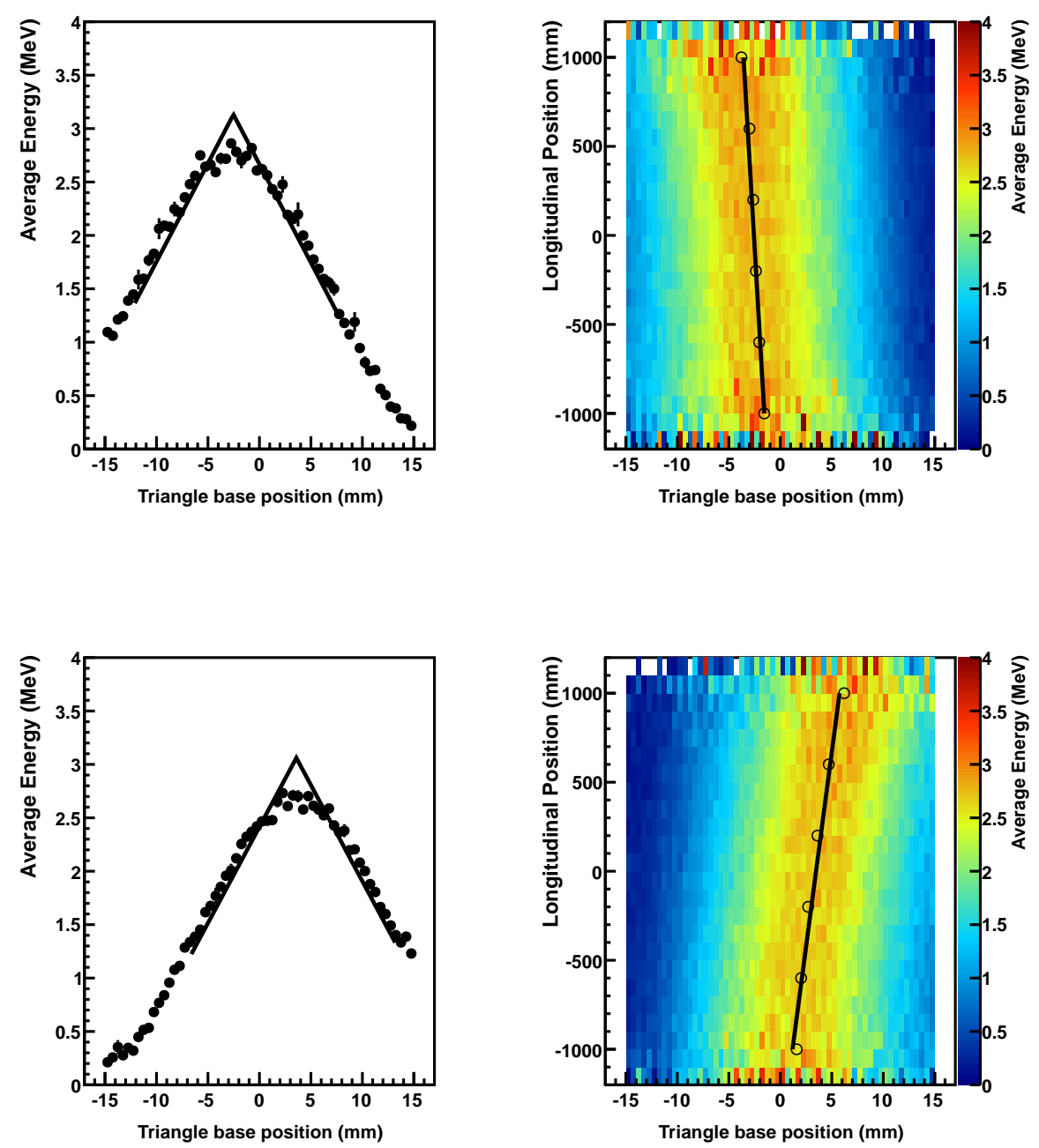

Figure 4.14: Alignment fits for module 50, plane 2 (top) and module 61, plane 1 (bottom). The peak is rounded due to energy deposited in the wavelength shifting fiber. The right side shows the plane rotaion fit. Figure from [20].

method converges after four iterations.

The constants are normalized to an average of 1.0. The constant $C_{s}$ for strip $s$ is

$$
C_{s}=\frac{\frac{1}{x_{s}}}{\frac{1}{N} \sum_{j} \frac{1}{x_{j}}}
$$


where $x_{s}$ is the truncated mean energy in strip $s$ and $\mathrm{N}$ is the number of channels in the detector.

A similar calibration constant is calculated for and applied to a plane as a whole (see Figure 4.15). The total strip-to-strip constant is a product of the individual strip and total plane corrections. The strip-to-strip calibration procedure, as well as the alignment calibration described in Section 4.2.2.2 are performed twice iteratively, using the corrections from the first iteration to improve those of the second. This calibration is performed when sufficient rock muon statistics are accumulated and can be motivated by detector hardware changes.

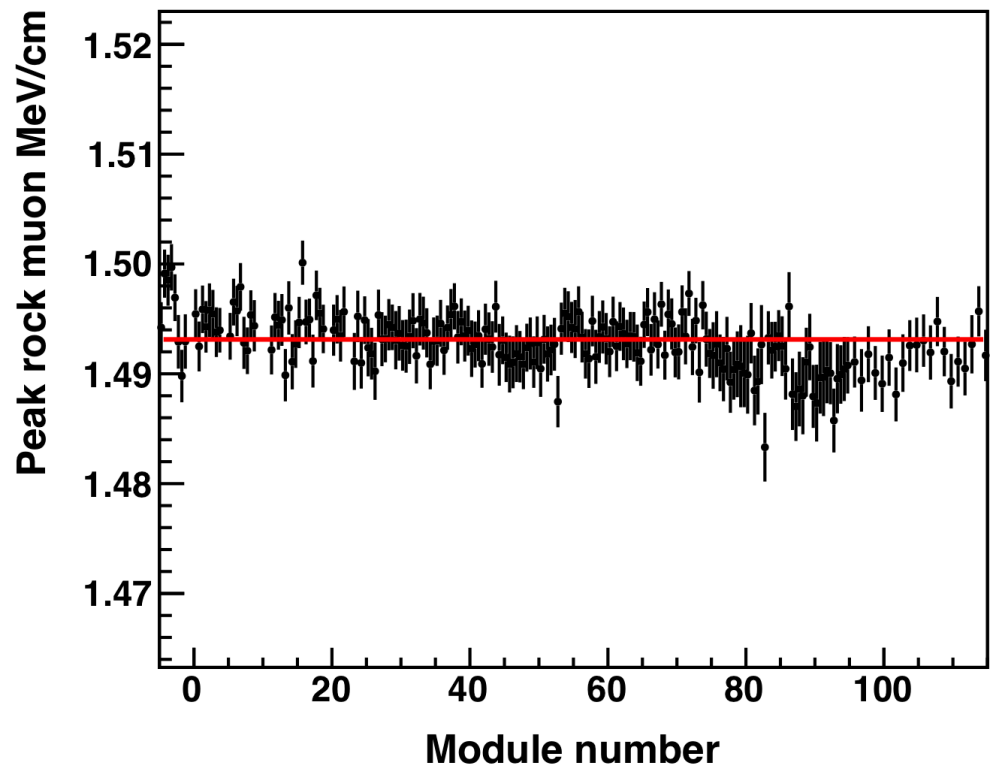

Figure 4.15: The fitted peak energy per unit path length for each plane. The peaks are fit to a line with zero slope. Figure from [20].

It is also in this calibration step that unresponsive or "dead" pixels are also identified. Dead pixels are typically caused by broken optical fibers or bad connections. 


\subsubsection{Absolute detector energy scale (Muon equivalent unit) calibration This} final energy calibration step converts PEs to energy. The factor that converts PEs to energy is the muon equivalent unit (MEU) ${ }^{1}$.

The calculation of the MEU factor begins with a sample of MINOS matched rock muons (i.e. rock muons with measured momentum). The positions and momentums of these rock muons are used as seeds of simulated rock muon events. Next, the reconstructed cluster (i.e. groups of hits in adjacent strips, see Section 4.3.2) energies of these data and simulation samples are compared.

The MEU factor, then is calculated

$$
F_{\mathrm{MEU}}=F_{\mathrm{MEU}, \text { trial }} \frac{E_{\mathrm{MC}}}{a} \frac{1}{E_{\mathrm{data}}}
$$

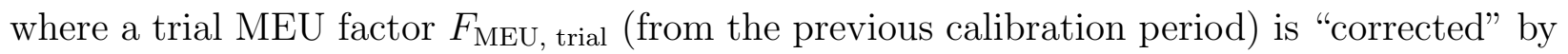
the simulation to data ratio of the peaks of the reconstructed cluster energy distributions $\frac{E_{\mathrm{MC}}}{E_{\mathrm{data}}}$, divided by $a$, the slope of the fit of simulated reconstructed cluster energy to the corresponding true cluster energy.

In this way, the MEU factor ensures data-simulation agreement on energy and PEs. A typical MEU factor is around $0.08 \mathrm{MeV} / \mathrm{PE}$.

Due to scintillator degredation, the peak muon cluster energy decreases with time as shown in Figure 4.16, but the MEU calibration corrects for the degredation and flattens the rock muon energy spectrum.

\subsubsection{Time calibration}

The final calibration step is a correction to timing offsets introduced by several detector components.

Three types of timing corrections are needed. First there is the straightforward time of flight in fiber correction, which depends on the hit location along the strip. Second, there are electronics offset corrections, which are fixed for each FEB. And third, there are time slewing delays from the scintillation and WLS fiber photon absorption process, as well as

\footnotetext{
${ }^{1}$ Also referred to ocasionally as the muon energy unit.
} 


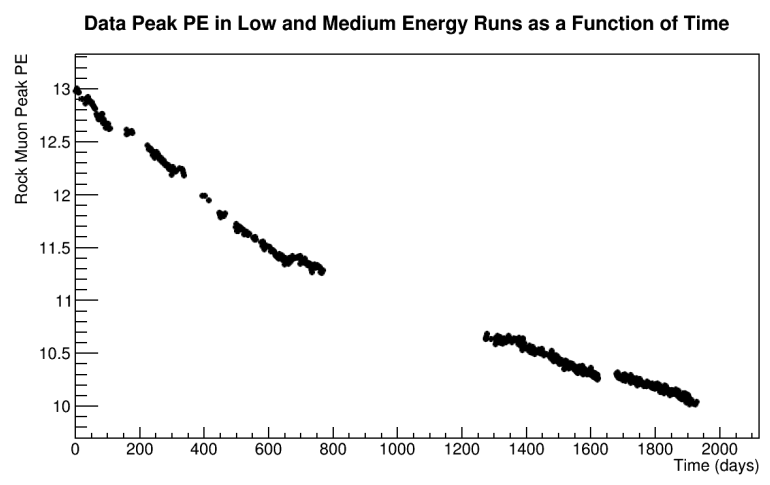

(a)

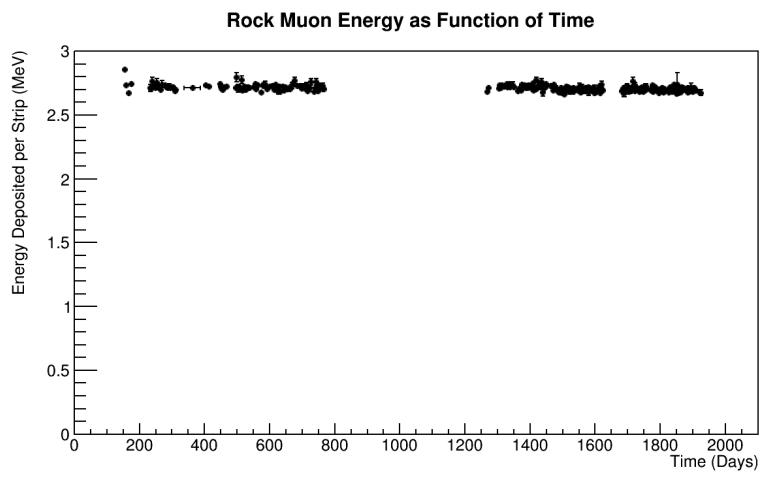

(b)

Figure 4.16: Top: Peak cluster energy decreases over time with the degredation of the scintillator. Bottom: The MEU calibration supplants the missing signal. The gap large gap between the data corresponds to the shutdown between the Low and Medium Energy eras.

from the possibility that the PEs arriving at the PMT may either proceed directly to the PMT or they may first travel in the opposite direction to be reflected off the WLS fiber's reflective coating. The calibrated time for a hit in strip $s$ is calculated:

$$
T_{\text {hit }}(s)=T_{\text {raw }}(s)-T_{\text {ToF }}-T_{\mathrm{FEB}}(s)-T_{\text {slew }}(P E)
$$

The FEB and slew corrections are calculated in situ with a through-going rock muon sample, 
and the procedure is performed iteratively to better separate the two effects. For muon tracks, the relative time between two hits can be determined from the muon speed and path length (which was previously calibrated by the procedure described in Section 4.2.2.2). First a reference time is formed from the truncated mean hit time along a rock muon track. Next, electronics offsets are calculated from the difference between hit time and reference time.

In the first iteration, the offset is used to measure the time slewing. In subsequent iterations, the previous electronics correction as well as the previous time slewing correction are used to improve the calibration. To calculate the time slewing correction, first the hits of all rock muons in the sample are binned by PEs. Time of flight and electronics corrections are applied, and then the peak of the difference of hit time to reference time is found with a fit. As a hit PE increases, it becomes increasingly likely that the first PE to reach the PMT traveled directly from the hit location, and thus the time slew correction decreases with PE. In the highest bin of $\mathrm{PE}$, the overal time correction approaches the electronics resolution of 2.2 ns.

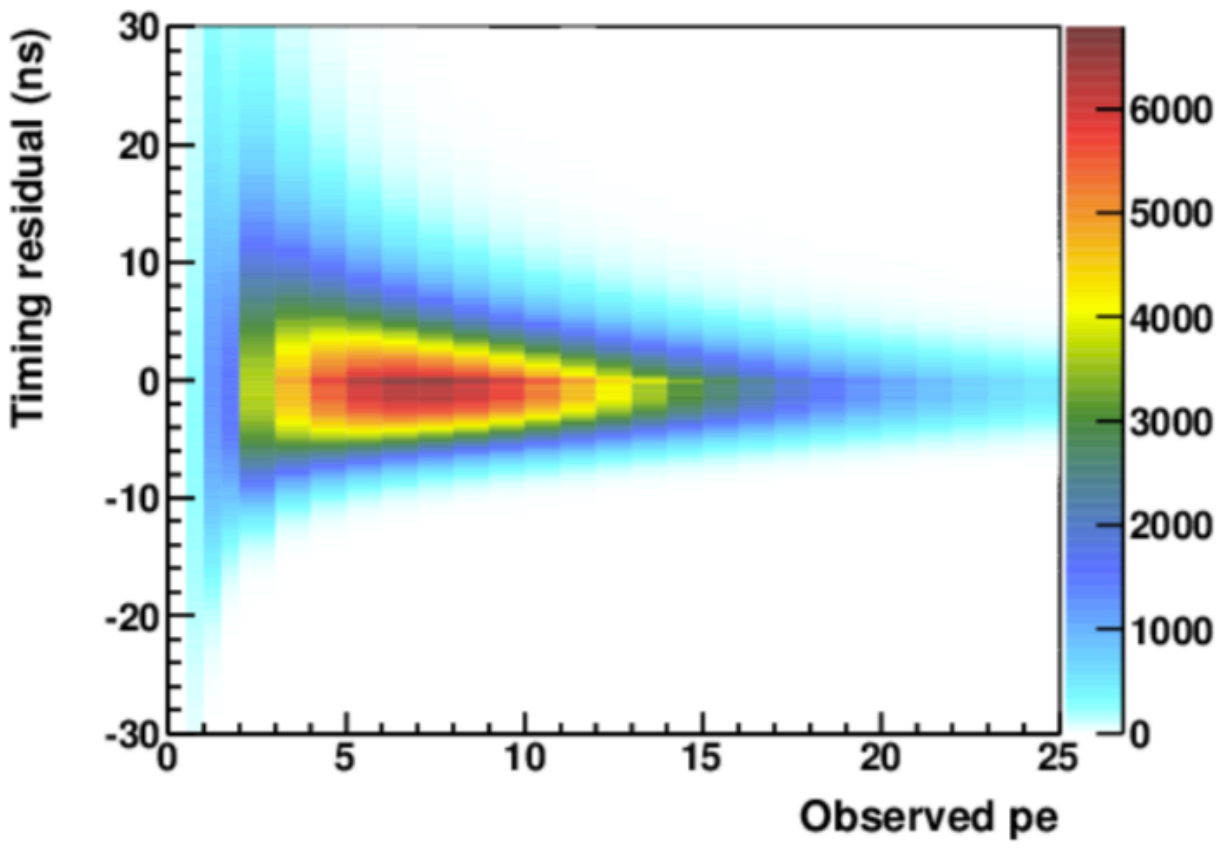

Figure 4.17: The timing residual as a function of PE after all calibrations. Figure from [82]. 


\subsection{DATA RECONSTRUCTION}

Calibrated data subsequently undergoes a series of common reconstruction processes that separates distinct neutrino interacts from each other in time, create particle track objects, and perform low-level event energy measurements

\subsubsection{Time slicing}

The NuMI beam is sufficiently intense to produce multiple neutrino interactions in a single 10 $\mu$ s spill. Time slicing is the first reconstruction procedure employed, and is used for certain calibration steps. Its purpose is to split a single readout gate in time into single neutrino interactions.

The slicer algorithm first arranges all gate hits in the detector by hit time. Next, it proceeds through the hits, summing activity as it goes. If the summed energy reaches a threshold of $10 \mathrm{PE}$, a time slice window is created, and activity falling within a buffer on either side of the window is included.

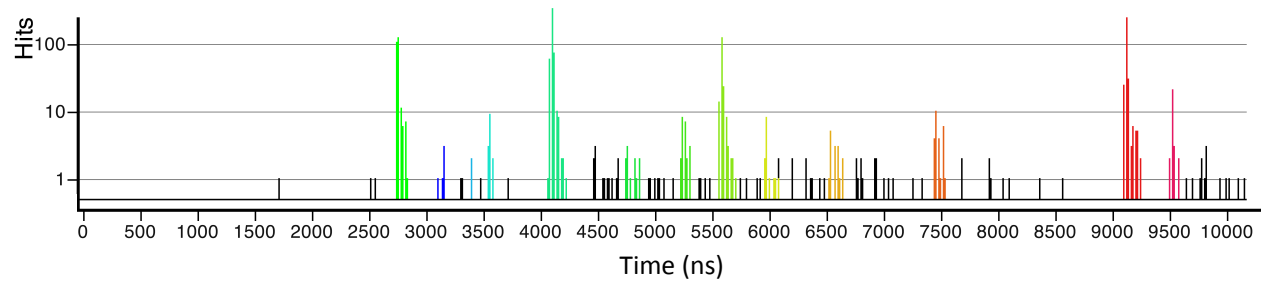

Figure 4.18: Colored time slices of $10 \mu \mathrm{s}$ of a single readout gate. Black entries are groupings below the threshold for forming a physics event. Figure from [20].

Intensity in the ME is sufficiently high, such that activity pile up has a significant effect on neutrino event reconstruction efficiency. Thus the slicer was retuned to be more aggressive. In the LE, the minimum time window was $80 \pm 30 \mathrm{~ns}$, while in the ME it has been shortened to $24 \pm 9$ ns. Figure 4.19 shows how an overlapping rock muon was separated by the new time slicing parameters. The time of flight through MINERvA for a particle traveling at the speed of light is $\sim 10 \mathrm{~ns}$, so the window minimum assumes some reconstruction time 
spread. Subsequent reconstruction and analysis steps of Chapter 6 are performed on neutrino interactions defined by time slices.
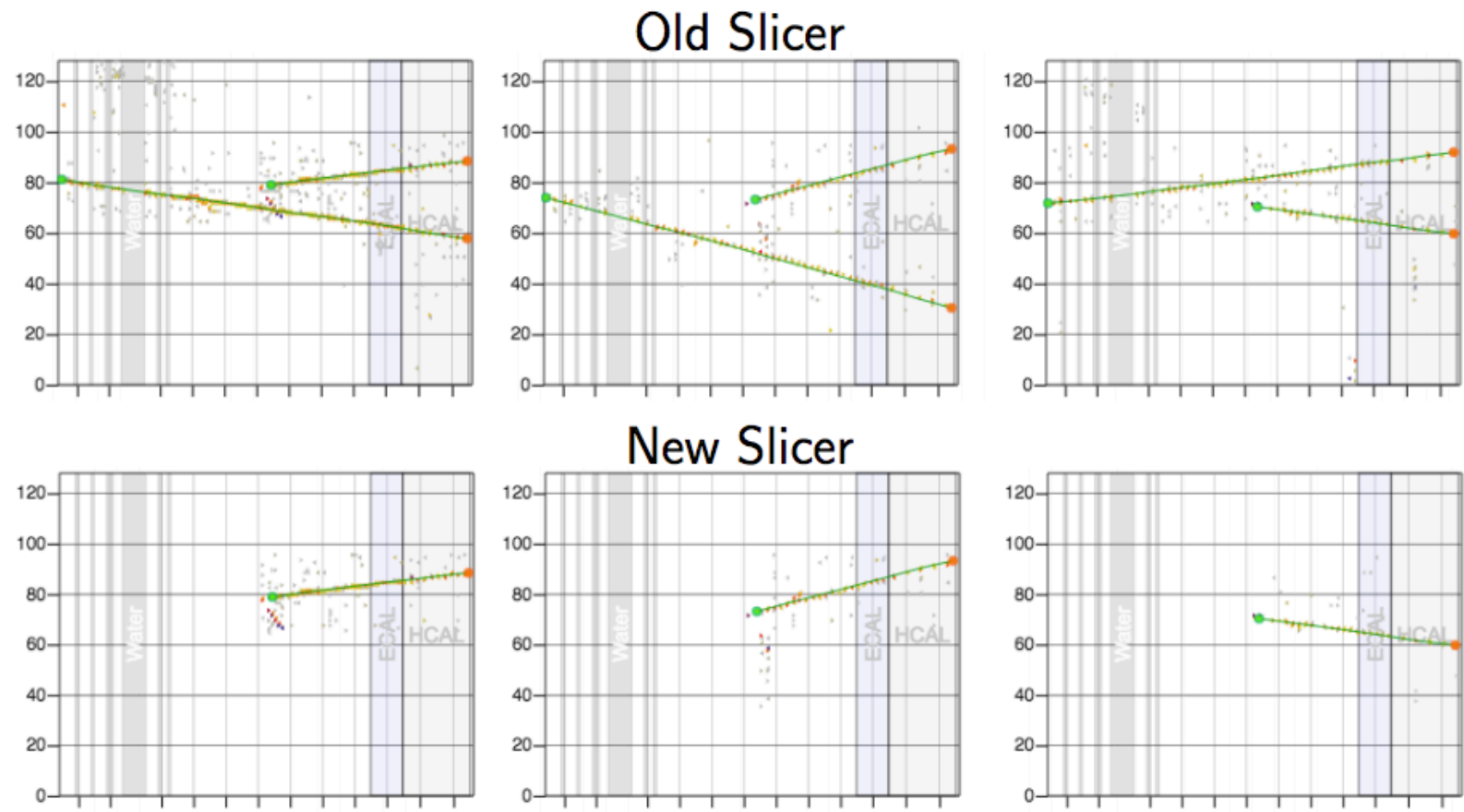

Figure 4.19: An overlapping rock muon is separated from a neutrino event with a tighter time slicing parameters. The three planes of each slice show the $\mathrm{X}, \mathrm{U}$, and $\mathrm{V}$ plane event projections with the plan number along the abscissa. For more details on the event display refer to Section 5.2. Figure from [51].

\subsubsection{Clustering}

Groups of one or more hits in adjacent strips within a plane are grouped into clusters. Cluster position is the energy-weighted average position, and cluster time is taken to be the time of the highest energy hit. Typically, a cluster is formed from two hits from a single particle's track and obtains $3 \mathrm{~mm}$ resolution. More generally, clusters can be categorized into five groups (shown in Figure 4.20) based on energy deposition patterns:

- Trackable From hits of at least $0.5 \mathrm{MeV}$ in 1-3 adjacent strips, and totalling 1-12 MeV. 
Typical of a minimum ionizing particle.

- Heavy ionizing Similar to trackable but with no upper energy limit. Typical of hadron track endpoint Bragg peak deposits.

- Supercluster At least $1 \mathrm{MeV}$ but otherwise not satisfying the trackable or heavy ionizing criteria. Typical of wide electromagnetic showers with energy spread across more than 4 strips.

- Cross talk A single, often low energy hit which is not physically adjacent to hits in other strips, but which is close to hits in electronics space on the PMT input face. Cross talk clusters are not included in later reconstruction steps.

- Low activity From hits with total energy less than $1 \mathrm{MeV}$. Used in recoil and hadronic energy measurement.

\subsubsection{Tracking}

Clusters are next further grouped into tracks. Tracks are formed by first grouping into track seeds: combinations of three clusters that fall in consecutive planes of the same $\mathrm{X}, \mathrm{U}$, or $\mathrm{V}$ view. Next, seeds of the same view with slope and intercept that are consistent with a $2 \mathrm{D}$ track are merged. Two-dimensional tracks in separate views are next stitched together into a 3D track, provided they are of the same longitudinal distance and are consistent with a 3D line. A Kalman filter is used to find re-scattered tracks.

Separately, tracks are reconstructed in the MINOS near detector. Matches between frontentering MINOS tracks, and rear-exiting MINERvA tracks are matched by slope, intercept and timing. MINOS-matched tracks are assumed to be muons, and they are promoted to the primary track, the upstream end of which defines a neutrino interaction vertex, called the primary vertex.

After identification of the primary vertex, a track search is re-performed, this time an-

chored around the vertex. Tracks identified this way are connected with the primary vertex, which is refit to the point of closest approach for all the tracks.

Low energy particles which traverse only a few planes are often not found by the above track reconstruction method, which requires a minimum of nine planes. Thus a separate 

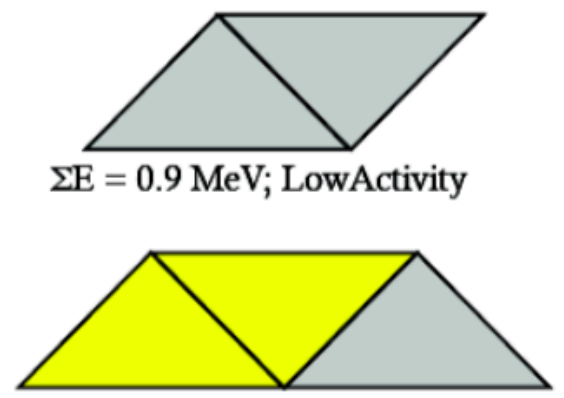

$\Sigma \mathrm{E}=10 \mathrm{MeV} ;$ Trackable
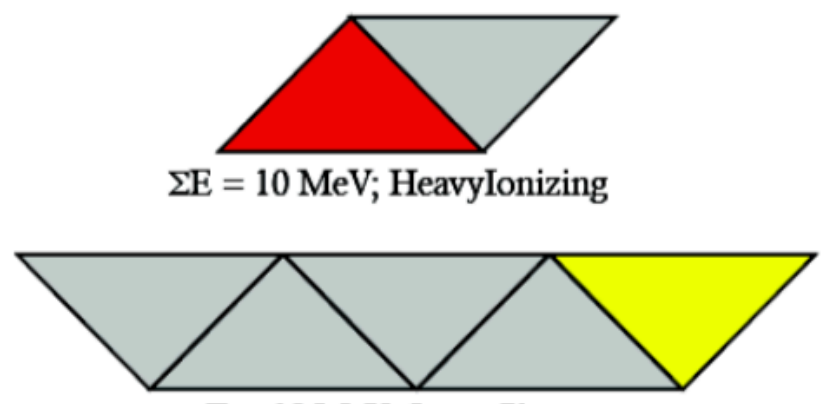

$\Sigma \mathrm{E}=10 \mathrm{MeV}$; SuperCluster

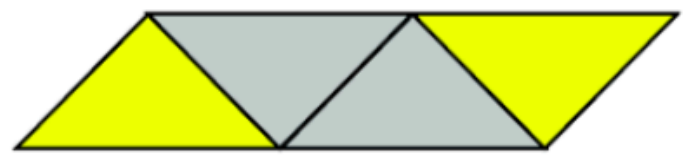

$\Sigma \mathrm{E}=5 \mathrm{MeV} ;$ SuperCluster

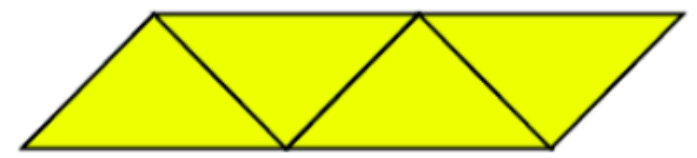

$\Sigma \mathrm{E}=6 \mathrm{MeV} ;$ SuperCluster

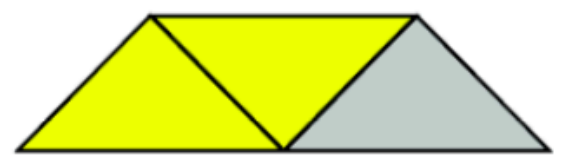

$\Sigma \mathrm{E}=17 \mathrm{MeV} ;$ HeavyIonizing

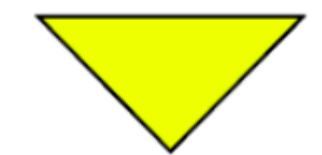

$\Sigma \mathrm{E}=5 \mathrm{MeV} ;$ Trackable

\begin{tabular}{|c|c|}
\hline Low E & $<1 \mathrm{MeV}$ \\
\hline Medium E & $1-8 \mathrm{MeV}$ \\
\hline High E & $8+\mathrm{MeV}$ \\
\hline
\end{tabular}

Figure 4.20: Examples energy deposition patterns and their cluster categories. Figure from G. Perdue.

short track reconstruction method that requires clusters in only four planes is also employed. With clusters in four planes the minimum information (an X point, Y point, dX/dZ slope, and $\mathrm{dY} / \mathrm{dZ}$ slope) is available to form a 3D track. Unlike the long track method, the short track method creates three dimensional seeds from two $\mathrm{X}$, one $\mathrm{U}$, and one $\mathrm{V}$ cluster. In a similar method to the long tracking, seeds are merged together to form 3D tracks where possible. Ultimately, short tracks are required to traverse a minimum of five planes. Five planes corresponsds approximately to $6.8 \mathrm{~cm}$ and a lower pion tracking threshold of $50 \mathrm{MeV}$ kinetic energy. Pion tracking efficiency as a function of momentum and angle are shown in Figure 4.22 . 

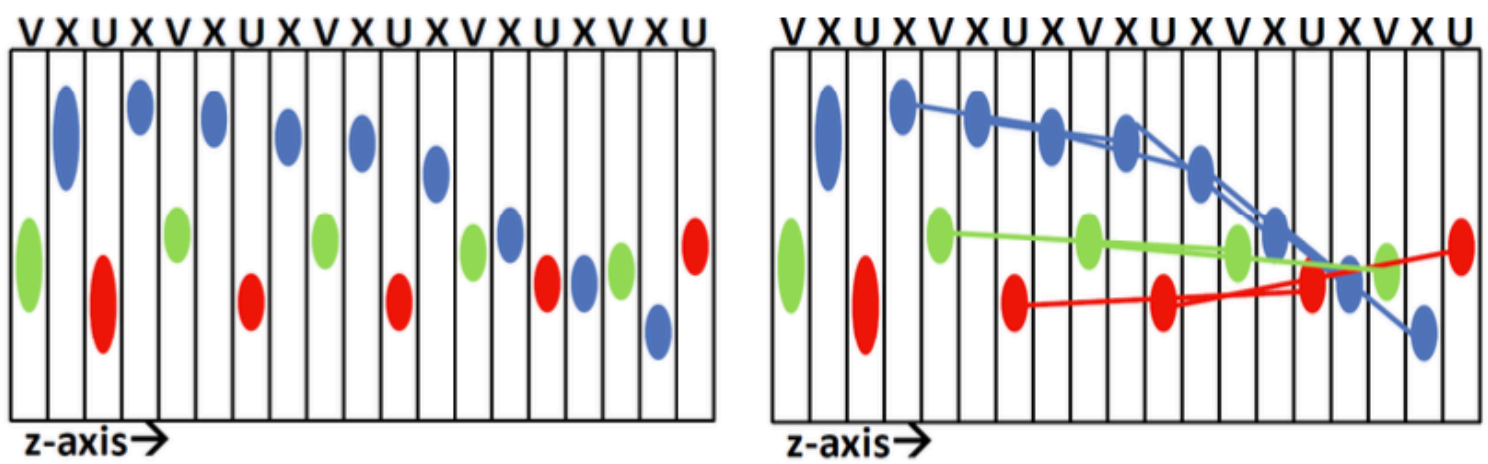

VXuXVXUXVXUXVXUXVXu
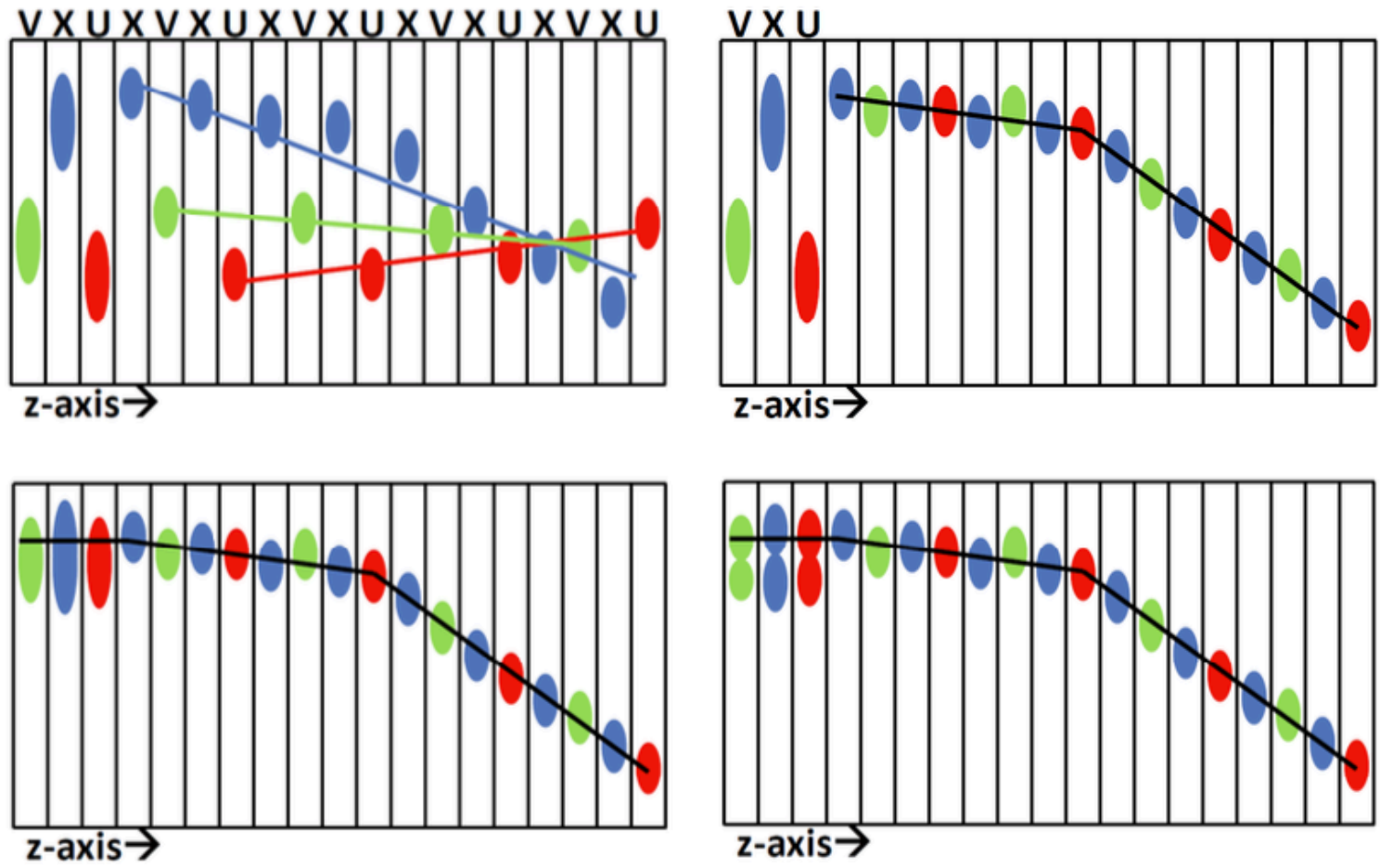

Figure 4.21: Illustration of the long tracking procedure. (TL) Clusters formed. (TR) Track seeds formed, superclusters not used. (ML) Seeds merged into candidates. (MR) 3D track formed. (BL) Superclusters added to track. (BR) Superclusters broken by cleaning procedure. Figure from [45]. 

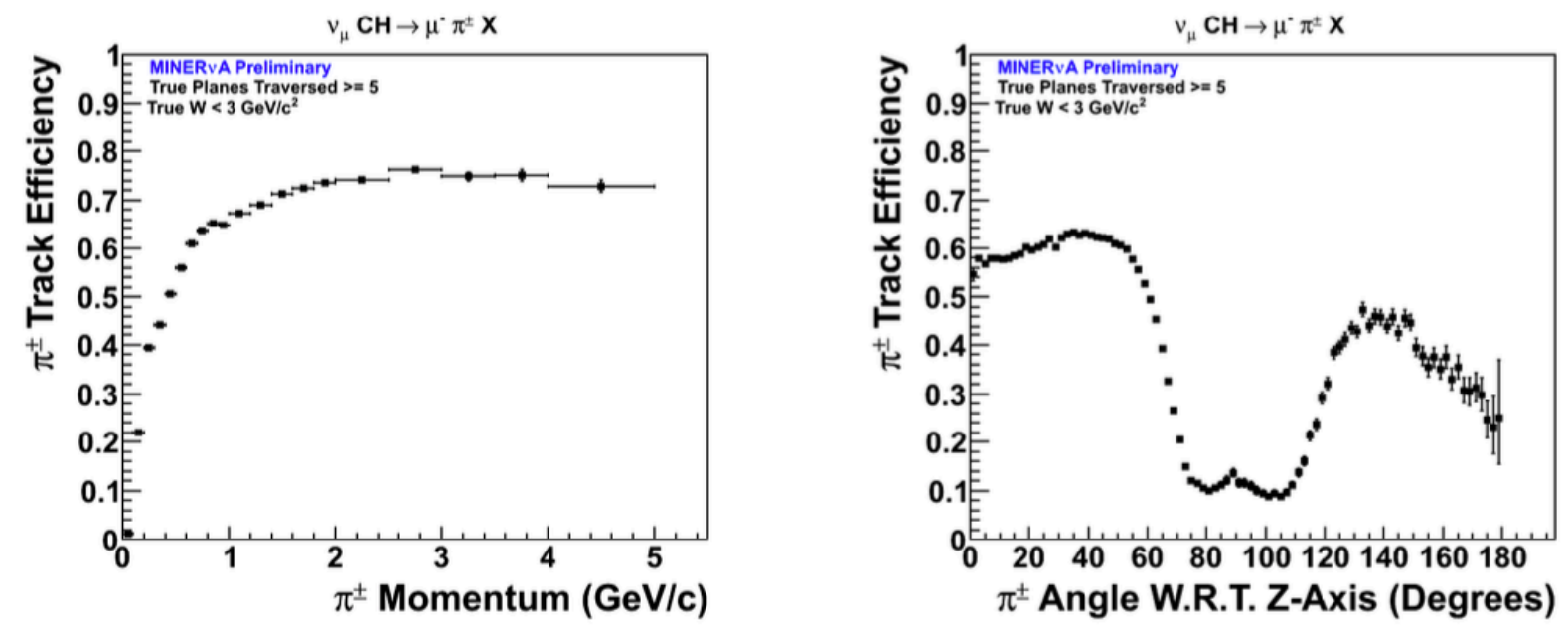

Figure 4.22: Pion tracking efficiency as a function of momentum and angle with respect to the z-axis. Inefficiencies are due predominantly to pion reinteractions. Figure from [45].

\subsubsection{Muon reconstruction}

This analysis studies a $\nu_{\mu}$ charged current interaction, which produce a $\mu^{-}$. As described in the previous section, an event's long track which is matched to MINOS is taken to be the muon signature of a charged current event. This is justified by the fact that very few nonmuonic particles exit the back of MINERvA due to the downstream electronic and hadronic containment calorimeters.

In MINOS, both the muon track distance and amount of curvature can be used to measure the muon momentum, while the direction of curvature signals the muon charge (and thus the neutrino or antineutrino nature of the interaction). When studying neutrinos, the magnetic field is aligned to bend muons towards the magnetic coil, providing the maximum track range from which to measure the momentum. Figure 4.6 shows the front face of the MINOS detector, including the magnetic coil hole and the beam centroid.

MINOS is a steel calorimeter, and it is not uncommon for muons to stop within the minos volume. From track range, muons momentum can be reconstructed to within $2 \%$. 
When muons exit the side of the detector or have enough energy $(\sim 10 \mathrm{GeV})$ to exit the back, momentum can be reconstructed from range (to within $\sim 2.6 \%$ ) according to:

$$
\frac{1}{R}=\frac{0.3 \times B \times q}{p}
$$

where $R$ is the radius of curvature, $B$ is the magnetic field, $q$ is the muon charge in units of electron charge, and $p$ is the muon momentum. A Kalman filter method of track reconstruction is similar to that employed in MINERvA, and it takes into account a magnetic field map, which is stable with time.

The muon energy calculated in MINOS must also be propagated upstream to obtain a momentum at the primary vertex. This energy is calculated from the Bethe-Bloch equation the parameters of which create some uncertainty on the final value.

\subsubsection{Hadronic recoil energy}

Hadronic recoil energy (referred to as $E_{\text {had }}$ or equivalently $E_{\text {recoil }}$ ) of the neutrino interaction, together with muon energy, are the two ingredients used to measure neutrino energy:

$$
E_{\nu, \text { reconstructed }}=E_{\mu}+E_{h a d}
$$

Measurement of the hadronic recoil energy is not as straightforward as summing the energy in the final state. This is because neutral particles in the final state often do not produce scintillation light and because charged particles can be below detection threshold.

Thus the hadronic energy is taken to be the sum of non-muon energy, adjusted by calorimetric correction from simulation that attempts to account for unseen energy. Relying on simulation to predict the amount of energy carried by neutral particles, the correction introduces model dependence, which we attempt to correct for with systematic errors (see Section 7.3).

The first step to the calorimetric correction is a straightforward correction due to energy deposited in passive materials:

$$
C^{i}=c^{i}+\frac{1}{f}
$$


where $C^{i}$ is the correction factor for subdector $i$ (corresponding, for example to the tracker, ECAL, and HCAL), $c^{i}$ is a constant for each subdetector measuring the fraction of seen to unseen energy due to a passive plane, and $f=0.8347$ is the fraction of active material in a tracking plane. For the tracker $c^{i}=0$.

The hadronic energy is then calculated:

$$
E_{h a d}=\alpha \times \sum_{i} C^{i} E_{i, \mathrm{vis}}
$$

where $\alpha$ is an energy-dependent scale that characterizes the neutral or otherwise undetected and uncontained energy. Figure 4.23 shows the fractional energy resolution on the reconstructed hadronic recoil energy.

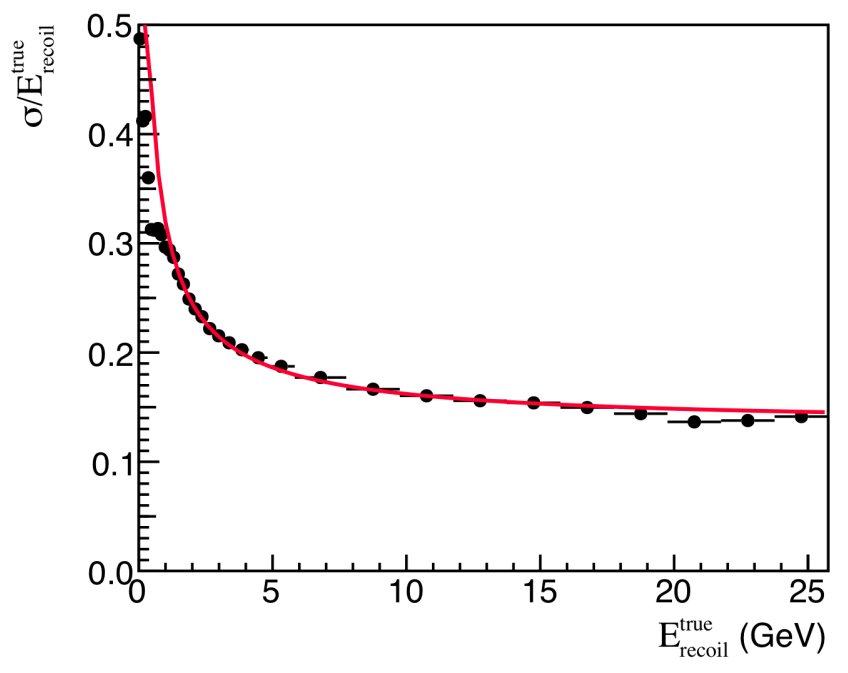

Figure 4.23: Fractional resolution on $E_{\text {recoil }}$. Resolution is worse at low energy where neutral energy constitutes a larger fraction of the total. Figure from [20]. 


\subsection{SIMULATION}

This section describes the simulation of neutrino interactions in the MINERvA detector and the generation of simulated data. The simulation begins from the output of the flux simulation (Section 3.2), it proceeds to simulate neutrino interactions inside of a MINERvA geometry model including the energy deposits of interaction products, and it ends with "precalibrated" raw ADC counts of hits in individual strips. At that point, the data format of simulated events is identical to that of raw data, and both simulation and data undergo the calibrations procedure described in Section 4.2.

\subsubsection{Neutrino interaction simulation}

A given amount of POT specifies the amount of simulated data to make. The flux simulation provides the identity, kinematics, and decay point of neutrino parent particles from single NuMI protons. From a thorough geometry simulation of the MINERvA detector (which includes everything from tracking planes, outer calorimeter and detector, nuclear targets, passive construction materials, and air gaps) a point is randomly chosen as the direction towards which the neutrino will be pointed. A weight capturing the probability that the neutrino would decay to that point is recorded (see Section 5.4). Along the neutrino direction of travel ray, the total interaction cross sections of the detector materials traversed are considered, and an interaction point and target material is chosen. Interactions are not generated in the downstream ECAL and HCAL due to computation limitations.

At this point, the GENIE event generator [27] takes over. GENIE simulates quasi-elastic

scattering, resonance production, deep inelastic scattering, and coherent pion production, all for charged and neutral currents (CC and $\mathrm{NC}$ ). Additional rare processes including $\mathrm{CC}$ charm production, neutrino-electron elastic scattering, and inverse muon decay $\nu_{\mu} \mathrm{e}^{-} \rightarrow \nu_{e} \mu^{-}$are also simulated.

The theoretical models of Section 2.3 that are implemented into this version of GENIE are described in the following Section 4.4.2. To choose an interaction process among those implemented, files containing the total cross sections for each process as a function of neutrino 
energy are referenced. Product particle kinematics are determined from interaction models.

\subsubsection{GENIE neutrino interaction simulation}

The GENIE event generator v2.12.6 [27] is used to model neutrino interactions, nuclear modifications, and final state interactions.

4.4.2.1 GENIE neutrino interaction models Charged current quasi-elastic interactions are modeled by Llewellyn-Smith with the pseudo-scalar and axial vector form factors of Section 2.3.1.1. For the axial vector form factor, a dipole form is assumed, and the axial mass has default value $0.99 \mathrm{GeV}$.

Deep inelastic scattering is modeled by an effective leading order calculation, including modifications from Bodek and Yang for scattering at low- $Q^{2}$ [38]. The Bodek-Yang modifications are scaled by to agree with very high energy neutrino scattering measurements $(100 \mathrm{GeV})$. Cross sections are computed for scattering off of individual partons.

Hadronization is implemented in the AGKY model[129]. At $W>3 \mathrm{GeV}$, the PYTHIA/JETSET $(\mathrm{P} / \mathrm{J})$ model is employed[115], while at $W<2.3 \mathrm{GeV}$ the KNO model is used [72]. In between, the two models are scaled linearly to form a continuous function. In terms of approximate corresponding neutrino energy, the $\mathrm{P} / \mathrm{J}$ model is not relevant until $E_{\nu}=$ $3 \mathrm{GeV}$, and at $9 \mathrm{GeV}$, contributions from the $\mathrm{P} / \mathrm{J}$ and $\mathrm{KNO}$ models are equal. The KNO model functions by generating a nucleon, and any number of kinematically available low-mass mesons. The total charged hadron multiplicity is tuned to data. After particle generation, the invariant mass is divided among the outgoing hadrons. The PYTHIA hadronization is a standard program for high-energy collisions, and uses a library of models for intial- and final-state parton showers, among other high-energy processes.

Rein-Sehgal [104] is implemented for CC and NC resonance production. Sixteen of the 18 resonance of the $\mathrm{R}-\mathrm{S}$ model are included. Interference between resonances is not included. Lepton mass is considered negligible in cross section calculations, though it is considered for

the product phase space. The default axial mass $M_{A}^{\text {res }}$ is $1.12 \mathrm{GeV}$ which is set by [74]. A $20 \%$ uncertainty on this parameter is used. 
The DIS model is extended to low- $Q^{2}$ and $E_{\nu}$ to predict nonresonant pion production. To avoid double-counting processes covered by both Bodek-Yang DIS and Rein-Sehgal resonance, $\mathrm{R}-\mathrm{S}$ is restricted to $W<1.7 \mathrm{GeV}$, and the $\mathrm{B}-\mathrm{Y}$ prediction is manually suppressed to match pion multiplicity total cross section measurementsin this region. As described in Section 5.4.3, B-Y non-resonant pion production is scaled down by $43 \%$ via the event weights of MnvGENIE v1.

The PCAC model of Rein and Sehgal is used for coherent pion production [105][106]. In this theory, low- $Q^{2}$ neutrino scatters can be compared to pion scattering data. GENIE uses pion scattering on protons and deuterium data.

\subsubsection{GENIE nuclear modifications and final state interactions GENIE simu-} lates the nucleus as a relativistic fermi gas (RFG) from Martini et al. [83] with the BodekRitchie nucleon high-momentum tail modification (lacking two-particle knockout) as described in Section 2.3.4.1. Pauli blocking suppression is calculated as a function of atomic mass $A$, and is otherwise based only on whether the outgoing nucleon momentum exceeds $k_{F}$. Resonance production is not subject to Pauli blocking.

GENIE does not simulate RPA or $2 \mathrm{p} 2 \mathrm{~h}$ processes by default, but they have been added in via event weights for the MnvGENIE v1 tuning procedure, described in Section 5.4.3. Random phase approximation effects are from [96] and [59]. Two particle-two hole effects are from [98, 60] with an empirical enhancement from [111]. These modifications are not applied to resonance production.

Final state interactions are simulated by the INTRANUKE module. INTRANUKE implements a hadronic cascade method as described in Section 7.2.2. More specifically, GENIE uses the $h A$ effective cascade model of INTRANUKE which attempts to capture a full cascade from a single interaction, and is constrained by data. See [27] for a further description of INTRANUKE. Interactions produced by FSI effects are also subject to interactions with the nuclear medium. 


\subsubsection{Detector simulation}

After GENIE simulates the neutrino interaction, particles are propagated through the detector with Geant4. Next, real data gates are overlaid on the simulated event to approximate the effects of overlapping events. Finally, the energy from the GENIE, Geant4, and data overlay steps are "de-calibrated" in simulated raw detector readout data. This simulated raw sample, can then undergo calibrations in parallel with real data.

\subsubsection{Energy propagation (Geant4) Final state particles and kinematics from GE-} NIE are next passed to a Geant4-based program [15] which simulates the track paths through detector planes including the downstream calorimeters, ionization energy deposited in strips, and elastic or inelastic particle collisions. Following their four-vectors, particles are stepped by $1 \mathrm{~mm}$ segments, and at each point an interaction or decay may occur. These probabilities are determined from the QGSP_BERT physics list. The Bertini (BERT) cascade model[32][62] of this list governs interactions below $10 \mathrm{GeV} / c$ momentum. Product particles above $10 \mathrm{GeV} / c$ are rare and simulated by the quark gluon string precompound (QGSP) model [69]. The QGSP_BERT implementation in Geant is tuned to hadron-nucleus scattering data. Note that the Geant stepping size $(1 \mathrm{~mm})$ is smaller than the width of a plane (17 $\mathrm{mm}$ ), and thus several hits may be simulated in a single strip.

4.4.3.2 Data overlay Pile-up, the overlapping activity of coincident neutrino interactions, can obstruct reconstruction steps such as tracking and vertexing. Pile-up is added to simulation by overlaying simulated events with random data gates.

Data overlay impacts the ensuing readout simulation and eventual simulation reconstruction. First, the absolute energy scale (MEU) factor of the data gate is used to de-calibrate the simulated energy (see next Section 4.4.3.3), resulting in time-dependent and generally lower simulated photon statistics. Second, if a channel was determined to be dead in the calibration of the data gate, that channel is also excluded from the remaining simulation steps and for simulation reconstruction. Dead channels can affect event reconstruction, deteriorating vertexing and sometimes causing rock muons to be identified as an authentic 
MINERvA neutrino interaction.

4.4.3.3 Readout simulation The readout simulation takes the true Geant4 detector energies and "de-calibrates" them to obtain raw ADC counts. The reason for this procedure is to make the simulation resemble real data as closely as possible, by attempting to start with raw ADC counts. Finally, data and simulation can be passed through an identical calibrations procedure (Section 4.2) in parallel. More detail on readout simulation can be found in [82].

The first step of de-calibrating the simulation is to convert true Geant4 strip energy into an estimate of scintillation light. This is accomplished with Birks' law, which gives an amount of scintillation light as a function of energy loss $d E / d x$ [34]. Birks' constant $\kappa_{B}$ was measured by the MINERvA testbeam experiment [58] and found to be $0.0905 \pm 0.014 \mathrm{~mm} / \mathrm{MeV}$.

Next, scintillation light is converted into photoelectrons incident on a PMT. This is accomplished with three de-calibration corrections and one smearing correction which is an effective correction to capture the uncertainty in the entire calibrations process. First, the absolute energy scale (MEU) correction is undone, effectively shifting the absolute energy scale of all simulated strips at once and converting energy into photoelectrons. Second, the strip-to-strip and plane-to-plane corrections are divided out, decohering the response of each strip to a known energy input. And the third de-calibration step adds attenuation to the number of photoelectrons. After, the number of photoelectrons is multiplied by a smearing correction: a random number sampled from a gaussian curve of $\mu=1$ and $\sigma=0.0557$. This smearing correction attempts to capture the uncertainty of the entire calibrations process a combination of the strip-to-strip uncertainty (1.5\%), some statistical uncertainty on gains (3-5\%), and other possible sources. The precise value of $\sigma$ was set by requiring the spread in rock muon cluster energy to agree in data and simulation. Finally, to account for statistical fluctuations, the final de-calibrated PE is sampled from a poisson distribution with mean equal to the Birks-suppressed PEs corrected by the above four effects.

The next step in de-calibration converts PEs into raw ADC counts. First a PMT simulation amplifies the signal and adds electronics noise and cross talk hits. As usual, the gain of the overlay gate is used. The amount of cross talk to add is estimated from a rock 
muon sample for each PMT. Finally, the tri-linear FEB constants are used to convert charge to ADC counts. The process ends before pedestals are re-added, though hits which do not exceed the pedestal mean by at least three standard deviations are suppressed, aligning the simulated lower PE detection threshold between data and simulation.

To de-calibrate the timing simulation, first hit times are rounded to the FEB clock time resolution of $2.2 \mathrm{~ns}$. Then in bins of PE, a random, effective time smearing from Figure 4.17 is applied to account for all timing calibration corrections.

The result is simulated, raw, pedestal-corrected ADC hits with uncalibrated time. These simulated events in parallel to real data, then undergo calibrations, reconstruction (as described in this Chapter), and finally analysis (described in the following chapters). 


\subsection{DATASET}

This chapter discusses the dataset considered in this thesis. First, a broad description of the data and collection period is given (Section 5.1). Next, the data format, organization and processing pipeline is summarized (Section 5.2). After, the simulated Monte Carlo (MC) dataset is discussed (Section 5.3). And finally, the chapter ends with a description of event weights applied to the MC dataset (Section 5.4).

\subsection{DATASET DESCRIPTION}

The dataset considered in this analysis was collected between September 2013 and February 2019 in the NuMI forward horn current (FHC, neutrino mode), medium energy (ME) configuration. MINERvA collected, processed, and validated data for $1.15 \mathrm{e} 21$ protons on target (POT) out of a total 1.24e21 delivered from the Main Injector. This analysis uses 1.05e21 POT of the good MINERvA data across playlists ME1A - ME1G and ME1L - ME1P. For comparison MINERvA collected 3.04e20 POT in the low energy (LE) configuration which ended in 2013.

With a flux of approximately $700 \nu_{\mu} / \mathrm{m}^{2} / 1 \mathrm{e} 6$ POT and MINERvA's hexagonal cross section of $2.2 \mathrm{~m}^{2}$, nearly 60 billion neutrinos passed through MINERvA every second during the ME era.

With hardware upgrades over the course of the ME run, the Main Injector eventually increased its intensity from $\sim 20 \mathrm{e} 12$ protons / spill up to more than 50e12 protons / spill. This led to an increase in the number of neutrino interactions in the fiducial volume per spill from approximately 0.05 to 0.2 by the end of the ME. Simulation predicts $\sim 50$ million total 


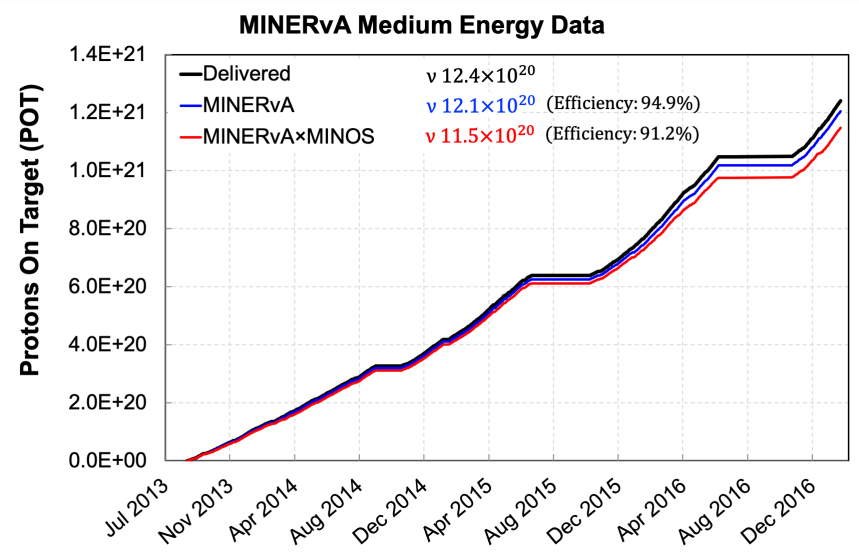

Figure 5.1: POT collected during the ME FHC neutrino-mode run. From [52].

neutrino events in the dataset, of which approximately 600,000 are signal.

\subsection{DATA FORMAT, ORGANIZATION, AND PIPELINE}

Several events may occur in a single spill or gate. There are typically 800 spills in a subrun and 40-50 subruns in a run. Runs are grouped into playlists, which are labeled alphabetically ME1A through ME1P. The size of each playlists varies between a few runs to more than 100 .

After minimal calibration and reconstruction, each event can be visualized as a 3D (plus some time information) image in MINERvA's proprietary in-browser event viewer, Arachne [120]. Arachne event displays are useful for developing intuition for subsamples or learning about the performance of reconstruction algorithms and event selection cuts. Figure 5.2 shows a simulated signal $\mathrm{CC} 1 \pi^{+}$event. The beam enters from the left, the shortest track is a pion, the longer track is a proton, and the longest track, exiting the back of the detector is a muon. Module number is listed along the abcissa, while strip number is along the ordinate. Observe the Bragg peaks for both hadron tracks. Hits with fewer than $2 \mathrm{PE}$ have been suppressed.

Figure 5.3 shows typical tracks and energy deposition signatures for the most common 
hadrons: protons, charged pions, neutrons, and neutral pions.

MINERvA data is stored in the ROOT tuple file format - event-by-event and typically in groups of subruns or runs. ROOT software provides a GUI browser to view event properties, called branches, in aggregate, which is useful for validation or straightforward studies.

After general reconstruction, the dataset is passed through analysis-specific reconstruction, which adds analysis-specific branches to the data. At this point, each event can be analyzed individually — cuts on event branches are performed, and subsamples can be visualized.

Reconstructed branches are organized into the Reconstruction tree. Branches that characterize the meta properties of the file's entire dataset, most importantly its POT count, are organized into the Meta tree. 


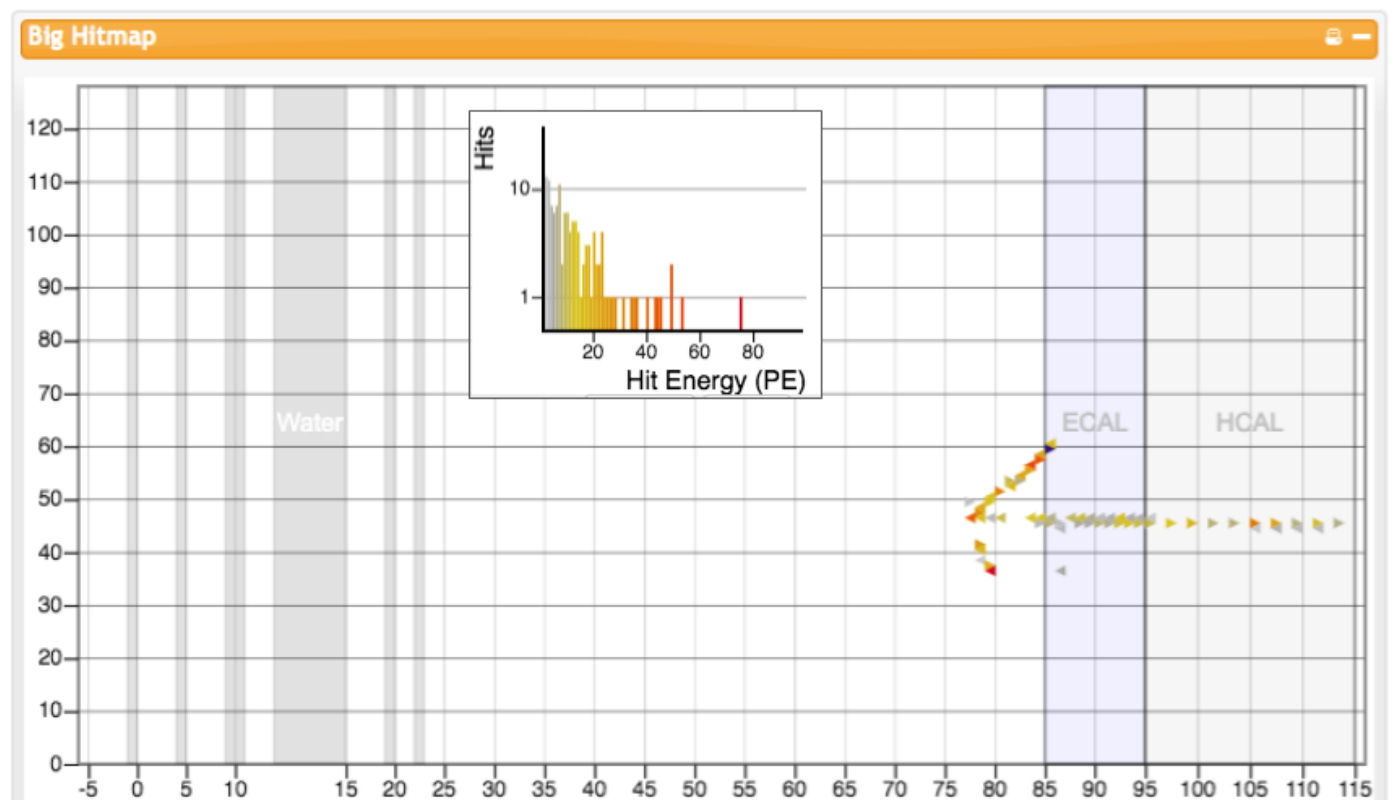

(a)

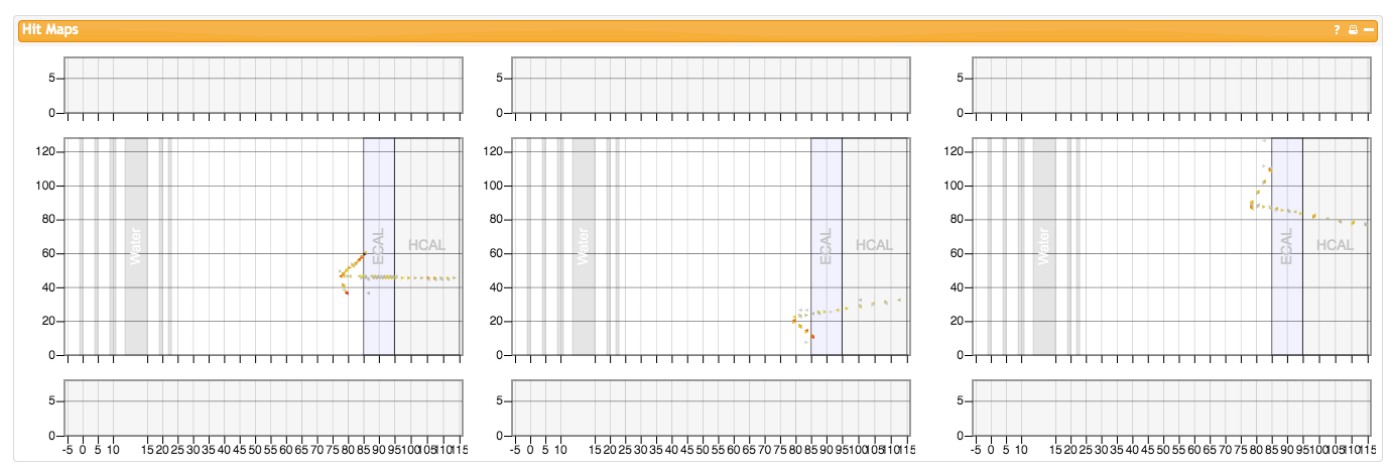

(b)

Figure 5.2: Arachne event display showing a simulated $\mathrm{CC} 1 \pi^{+}$event. (Top) X-view with hit energy scale. (Bottom) X-, U-, and V-views with outer detectors. 


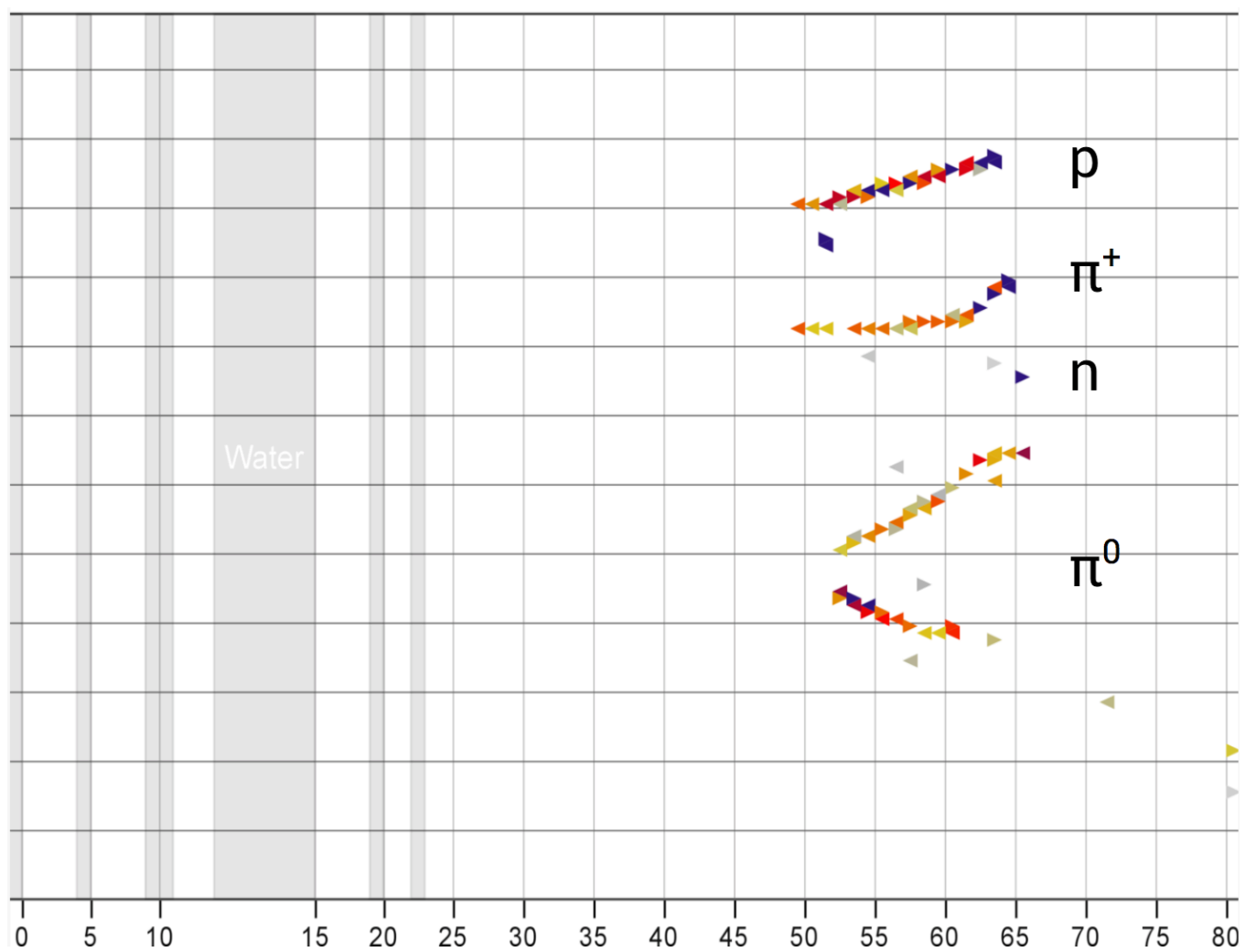

Figure 5.3: Arachne display of simulated hadron tracks. The proton, neutron, charged pion, and neutral pion are the most common hadrons in MINERvA interactions. Note the higher Bragg peak for the proton compared to the pion. Neutron energy is usually missed completely. The $\pi^{0}$ signature is its di-photon decay. Darker color indicates higher energy hits.

\subsection{SIMULATED MONTE CARLO DATA}

Alongside the experimental data, MINERvA generates Monte Carlo simulated data ("MC") as explained in Section 4.4. At each step of the data pipeline, the MC event-by-event tuple file format is identical to that of the data, so it can be treated on equal footing. As with data tuples, MC tuples also contain a Meta tree with POT information as well as a Reconstruction tree, whose branches have been filled by the identical reconstruction stage as the data. Unlike the data tuples, MC tuples also contain a Truth tree, which hold generator-level information 
about all simulated interactions, including events that could not be reconstructed. The reconstructed $\mathrm{MC}$ tree contains a subset of the events in the truth tree.

To reduce statistical uncertainties, the MC dataset is generated with approximately two times more incident protons on target. The MC is organized in the same ME1A - ME1P playlist format as the real data. The MC playlists used in this analysis contain $2.33 \mathrm{e} 21$ of the $2.46 \mathrm{e} 21$ POT of the full MC MINERvA dataset.

Table 5.1: Information contained in each data file tuple tree

\begin{tabular}{l|cccc} 
Tree & $\begin{array}{c}\text { Reconstructed } \\
\text { (data) }\end{array}$ & $\begin{array}{c}\text { Reconstructed } \\
(\mathrm{MC})\end{array}$ & Truth & Meta \\
\hline $\begin{array}{l}\text { Reconstructed } \\
\text { Branches }\end{array}$ & $\mathrm{X}$ & $\mathrm{X}$ & $\mathrm{X}$ \\
\hline $\begin{array}{l}\text { GENIE Simulated } \\
\text { Branches }\end{array}$ & & $\mathrm{X}$ & $\mathrm{X}$ & \\
\hline Event Weights & & $\mathrm{X}$ & $\mathrm{X}$
\end{tabular}

\subsection{EVENT WEIGHTING}

Producing Monte Carlo simulated data is computationally expensive. Event reweighting is a method used to update already-generated MC to accommodate improved theoretical models or otherwise data-motivated adjustments to $\mathrm{MC}$ input parameters. When filling event histogram distributions, each event's contribution to a bin is scaled by an event weight - the probability of the event occurring according to the various constraints.

The weights applied in this analysis are a product of flux, MINOS, and GENIE weights described in the following sections.

\subsubsection{Flux weights}

The MC is initially simulated with the G4NuMI v6r0 $\nu_{\mu}$ flux. Significant improvements on top of this version have been made in the simulation's pC hadron production (see Section 3.2) 


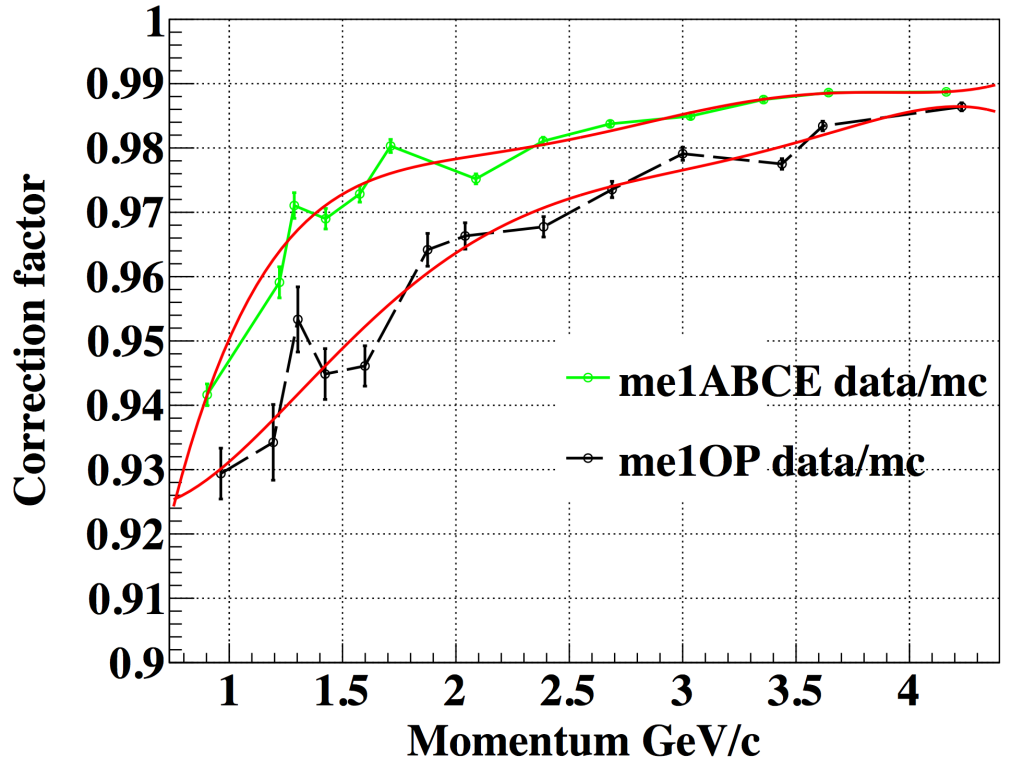

Figure 5.4: Correction to the MINOS tracking efficiency for the low-intensity playlists (A-D) and high-intensity playlists (OP). From [55]

and focusing horn geometry [79]. Additionally, flux weights from the incident proton beam spot size (see Section A.1) and magnetic horn tilt are playlist-dependent weights that reflect small, unintended changes in the NuMI beamline that occurred during data taking [67].

\subsubsection{Muon tracking efficiency}

The efficiency with which muons are tracked in MINOS is overestimated, perhaps because of a mismodeling in the MINOS data overlay simulation. Using in situ rock muon data, the mismodeling is weighted as a function of muon momentum and the NuMI batch intensity.

\subsubsection{MnvGENIE v1}

MINERvA uses a standard set of weights referred to by the label MnvGENIE v1 that modify the simulation physics engine GENIE from its default version 2.12.6. They tune the following processes: 
- Non-resonant pion production Based on a fit to pion production data on deuterium from bubble chamber experiments [112], the normalization of non-resonant pion production is reduced to $43 \%$ of its default value.

- Quasi-elastic scattering Two weights adjust the rate of quasi-elastic processes. The first adds the Random Phase Approximation (RPA) model [96][59] as a function of energy and three-momentum transfers $q 0$ and $q 3$. The second modifies the QE-like interactions of multi-nucleon pairs ("2p2h") to match MINERvA inclusive neutrino scattering data [111]. These processes produce no pions, they constitute a $<1 \%$ contribution to the selected events, and the effect of their weights on the event rates is very small.

The physical processes behind these weights were described in Section 2.3, while the GENIE default model and these modifications are described in more detail in 4.4.2. 


\subsection{CHARGED PION DIFFERENTIAL CROSS SECTION MEASUREMENT}

In this chapter, an overview of the cross section calculation is given (Section 6.1), followed by a description of the signal definition (Section 6.2), and subsequent discussions of each analysis component is given in turn: event selection (Section 6.3), background subtraction (Section 6.4), unfolding (Section 6.5), and efficiency correction and normalization (Section $6.6)$.

Systematic uncertainties on the measurements are presented in Chapter 7, and cross section results are presented in Chapter 8.

\subsection{OVERVIEW OF THE MEASUREMENT}

The differential cross section with respect to an event variable $x$ and in some bin $\alpha$ is given by:

$$
\left(\frac{d \sigma}{d x}\right)_{\alpha}=\frac{\sum_{j} U_{j \alpha}\left(N_{d a t a, j}-N_{s i m, j}^{b k g d}\right)}{A_{\alpha}(\Phi T) \Delta x}
$$

where $N_{d a t a, j}-N_{s i m, j}^{b k g d}$ is the number of observed events minus the predicted background events in detector bin $j$. The matrix $U_{j \alpha}$ is the unfolding matrix which transposes from observed bin $j$ of $x$ into "true" bin $\alpha$. $T$ is the number of target nuclei in the detector, $\Phi$ is the incident neutrino flux, and $A_{\alpha}$ is a simulated detection efficiency and acceptance correction.

The cross section measurement proceeds as follows: 
1. Event selection In this stage, the data is passed event-by-event through a series of cuts designed to select events whose properties satisfy the signal definition and to remove all others. Cuts can be optimized on several criteria, such as their capacity to maximize the signal selection efficiency and signal purity, to minimize systematic uncertainties, or to select events that have better kinematic reconstruction quality. Event selection places each event into kinematic bins, and the collection of selected events is represented as a histogram. Subsequent steps of the cross section calculation manipulate the event selection histograms and pass them to the next stage.

2. Background subtraction After event selection cuts, background events - events that pass selection cuts, but which are not in fact signal - remain in the sample. In this stage, these events are removed. A binned background sample is obtained from simulation by passing the Monte Carlo dataset through event selection cuts (which are only sensitive to reconstructed event properties) and then referring to their truth properties to determine if the event is signal or background. The background is subtracted, bin-by-bin, from the event selection data distributions. The output is a background-subtracted data distribution.

3. Unfolding Due to shortcomings in detection and event reconstruction, the variables measured are smeared away from their true values. In the next stage of the cross section calculation, the smearing is undone in a process called unfolding. Here, an unfolding matrix, constructed from the simulation's discrepancies between reconstructed and true quantities, is applied to the background-subtracted data distribution, transforming it from a reconstructed variable into a "true" variable.

4. Efficiency (and Acceptance) Correction In this stage, the background-subtracted and unfolded sample is repopulated with the signal events that were lost due to inefficient selection cuts and to the geometric acceptance or kinematic thresholds of the detector. The efficiency and acceptance are simulated together as the number of selected signal events, divided by the total number of signal events, all in bins of the cross section variable.

5. Normalization The final stage normalizes the efficiency-corrected distributions by two scalar quantities and introduces cross section units of measure. The first scale factor is the 
muon neutrino flux exposure of the dataset. The second scale factor is the number of target protons and neutrons in the allowed neutrino interaction regions (i.e. fiducial volume) of the MINERvA detector. The distribution obtained after normalization must finally be scaled by the width of each bin to obtain a differential cross section measurement and giving the distribution a final unit of measure $\left[\mathrm{cm}^{2} /\right.$ target nucleon/[variable unit]].

A software package was built to process the data through this calculation, the output of which is a differential cross section distribution as a function of variables that categorize the event. The variables analyzed include: pion kinetic energy $T_{\pi}$ and pion angle with respect to the incident neutrino $\theta_{\pi}$, muon momentum $p_{\mu}$ and muon angle with respect to the incident neutrino $\theta_{\mu}$, experimental invariant hadronic mass $W_{\text {exp }}$, four-momentum transfer $Q^{2}$, and incident neutrino energy $E_{\nu}$. In parallel with the data cross section calculation, the Monte Carlo simulated dataset is also passed through the calculation pipeline. The Monte Carlo processing is both used for simulated inputs to the data cross section calculation, and it is also treated on equal footing as the data in order to obtain data-Monte Carlo comparisons for each stage of the calculation, including for the final cross section.

\subsection{SIGNAL DEFINITION}

The signal definition both defines the physical process that the analysis attempts to measure and it also delimits the process to a phase space that the detector and analysis are capable of the measuring.

The first set of criteria, which define the physical process that the analysis attempts to measure, require that the event:

- be caused by an incident muon neutrino that interacted via the charged current,

- have exactly one positively charged pion and zero other mesons exit the target nucleus, and

- have reconstructed invariant hadronic mass, $W_{\text {exp }}$ less than $1.4 \mathrm{GeV}$. $W_{\text {exp }}$ is defined in Equation 6.4, below. 
The second set of criteria, which define the detector's geometric and acceptance thresholds, require the event to have:

- interaction vertex within the fiducial volume of the detector.

- muon angle less than 20 degrees with respect to the incident neutrino beam.

- pion kinetic energy greater than 35 and less than $350 \mathrm{MeV}$.

The requirement to have the event's experimental invariant hadronic mass $W_{\text {exp }}$ less than $1.4 \mathrm{GeV}$ focuses the measurement on $\Delta(1232)$ resonance pion production, as well as nonresonant pion production which is expected to have largest effect on the rising edge of the $W$ resonance peak.

This requirement on $W_{\text {exp }}$ also belongs with the second set of criteria. The properties that need to be measured in this analysis, specifically the hadronic energy and the pion kinematics, become increasingly difficult to reconstruct as the $W$ increases. ${ }^{1}$ At high- $W$ more energy goes into neutral particles which are often not detected and, as a result, hadronic energy determination suffers. Also, beginning near $W_{\text {exp }} \sim 1.4 \mathrm{GeV}$, high hadron track multiplicity ( $>3$ tracks) begins to challenge MINERvA's position resolution, and track overlap deteriorates particle identification as well as track length, angle, and momentum reconstruction.

The fiducial volume includes most of the tracker region. It is defined as the $865 \mathrm{~mm}$ apothem regular hexagonal volume stretching from upstream module 25 at MINERvA zposition $5900 \mathrm{~mm}$ to downstream module 81 at z-position $8430 \mathrm{~mm}$. Interaction vertices must fall within the detector for several reasons. Most importantly, the muon and pion tracks must both originate from the same point, to confirm (provide very strong evidence) that they were born from the same neutrino and not some upstream process. Additionally, for the purposes of accurate energy reconstruction and particle identification, the full track lengths of the muon, pion, and other particles must be known. This analysis measures the cross section on scintillator and thus the fiducial volume region excludes the upstream target region, the downstream HCAL and ECAL, and the outer detector.

\footnotetext{
${ }^{1}$ To be precise, $E_{\text {had }}$ is not measured directly, but inferred from the visible energy and corrected for lost neutral and passive material energy, by model. This procedure is described in Section 4.3.5.
} 
Muon acceptance decreases as a function of muon angle with respect to the z-axis. Outside of 20 degrees, the efficiency drops quickly to zero. For future analyses, a z-dependent angle restriction may be considered, for efficiency is higher at downstream z positions and lower at upstream z-positions.

The lower and upper thresholds on pion tracking are 35 and $350 \mathrm{MeV}$. At the lower end, in order for MINERvA's reconstruction tracking algorithm to construct a track, hits in three adjacent planes are required. Thus the kinetic energy required for a pion to traverse three planes is $35 \mathrm{MeV}$. At the upper kinetic energy threshold, pions with kinetic energy greater than $350 \mathrm{MeV}$ can exit the back of MINERvA and thus no michel electron (the required signature of a positively-charged pion, see Section 6.3.3.1) can be observed. For future analyses, a restriction depending on the interaction z-position should be considered.

\subsubsection{Analysis variable definitions}

The differential cross section measurement will be measured as a function of the following variables: $T_{\pi}, \theta_{\pi}, P_{\mu}, P_{\mu}^{z}, P_{\mu}^{t}, \theta_{\mu}, E_{\nu}, Q^{2}$, and $W_{\exp }$.

The angles $\theta_{\pi}$ and $\theta_{\mu}$ are measured with respect to the incident neutrino direction.

The longitudinal and transverse muon momenta $P_{\mu}^{z}$ and $P_{\mu}^{t}$ are defined with respect to the incident neutrino direction. Only systematic errors and final cross section results are shown for these variables.

The neutrino energy $E_{\nu}$, is meaured as the sum of the leptonic (muonic) and hadronic system energies

$$
E_{\nu}=E_{\mu}+E_{h a d}
$$

where the measurements of $E_{\mu}$ and $E_{\text {had }}$ were previously described in Sections 4.3 .4 and 4.3.5.

The four-momentum transfer is defined purely by the lepton system:

$$
Q^{2}=-\left(p_{\nu}-p_{\mu}\right)^{2}=2 E_{\nu}\left(E_{\mu}-P_{\mu}^{L}\right)-\mathrm{m}_{\mu}^{2}
$$

where $p_{\nu}$ and $p_{\mu}$ are the neutrino and muon four-momentum, and $P_{\mu}^{L}$ is the longitudinal muon three-momentum relative to the incident neutrino direction. 
The generator invariant hadronic mass $W$ is distinguished from an experimental invariant hadronic mass $W_{\text {exp }}$ :

$$
\begin{aligned}
W^{2} & =\left(p_{\nu}+p_{N}+p_{\mu}\right)^{2} \\
W_{\text {exp }}^{2} & =m_{N}^{2}+2 m_{N}\left(E_{\nu}-E_{\mu}\right)=m_{N}^{2}+2 m_{N} E_{\text {had }}-Q^{2}
\end{aligned}
$$

where, to derive $W_{\text {exp }}$ from $W$, the initial momentum of the target nucleon is assumed to be zero and the four-momentum $p_{N}=m_{N}$. Of course, the target nucleon momentum is typically not zero, but this variable nonetheless is defined in terms of directly measurable quantities, and it can thus be compared to other experiments and studied by theorists and model builders. The invariant hadronic mass from the generator is not generally measurable by experiment. While in the case of a clean $p \pi$ final state, the invariant hadronic mass $W$ (post-FSI) could be determined, MINERvA's efficiency for reconstruction three final state tracks $\left(\mu, \pi^{+}, p\right)$ is very low.

The decision to define the signal based on $W_{\text {exp }}<1.4 \mathrm{GeV}$ (rather than on generator $W$ ) in order select $\Delta(1232)$, is motivated by ${ }^{2}$ the fact that, perhaps unexpectedly, $W$ very closely resembles $W_{\text {exp }}$ when $W_{\text {exp }}$ has been calculated with true quantities. In other words, the assumption that the target nucleon is at rest largely holds. ${ }^{3}$ The trouble instead, turns out to be that the reconstruction of $E_{\text {had }}$ is very bad, and through Equation 6.4 it is solely responsible for ruining agreement between $W_{\text {exp }}$ from reconstructed variables and $W_{\text {exp }}$ from true variables (and thereby $W$ from generator).

One shortcoming of the $E_{\text {had }}$ measurement, as described in 4.3.5, is that it bluntly takes $E_{\text {had }}$ to be the sum of all non-muon energy in the event. Assuming a pion track is always required, a better method would be:

$$
E_{h a d}=T_{\pi}+m_{\pi}+E_{\mathrm{rem}}
$$

where $T_{\pi}$ is determined from the track energy and $E_{\text {rem }}$ is the remaining non-muonic energy in the event. In the case of straightforward neutrino resonance production $\Delta^{++} \rightarrow \mathrm{p}+\pi^{+}$, true

\footnotetext{
${ }^{2}$ Besides the fact that $W$ is not directly measurable!

${ }^{3}$ It is nonetheless imporant to keep in mind that $W$ is not the same as $W_{\text {exp }}$, and the extent to which a cut on $W_{\text {exp }}<1.4 \mathrm{GeV}$ does or does not select $\Delta(1232)$ baryons exactly as $W<1.4 \mathrm{GeV}$ would is not known. Theorists are eager to see a measurement without a $W_{\text {exp }}$ restriction, but for reasons described at the beginning of this section, it is hard to do.
} 
$E_{\text {had }}$ is the kinetic energy of the proton (its rest mass existed prior to the reaction) plus the total (kinetic plus rest) energy of the pion (its rest mass did not exist prior to the reaction). Thus the pion mass must be added, while it is assumed that all proton kinetic energy is visible (and this is certainly not always the case). By separating the pion from the rest of the hadronic energy, the simulation would then correct neutral/unseen energy and specify uncertainties separately for each component, hopefully improving the $E_{\text {had }}$ measurement. This alternate $E_{\text {had }}$ measurement will be considered in future work.

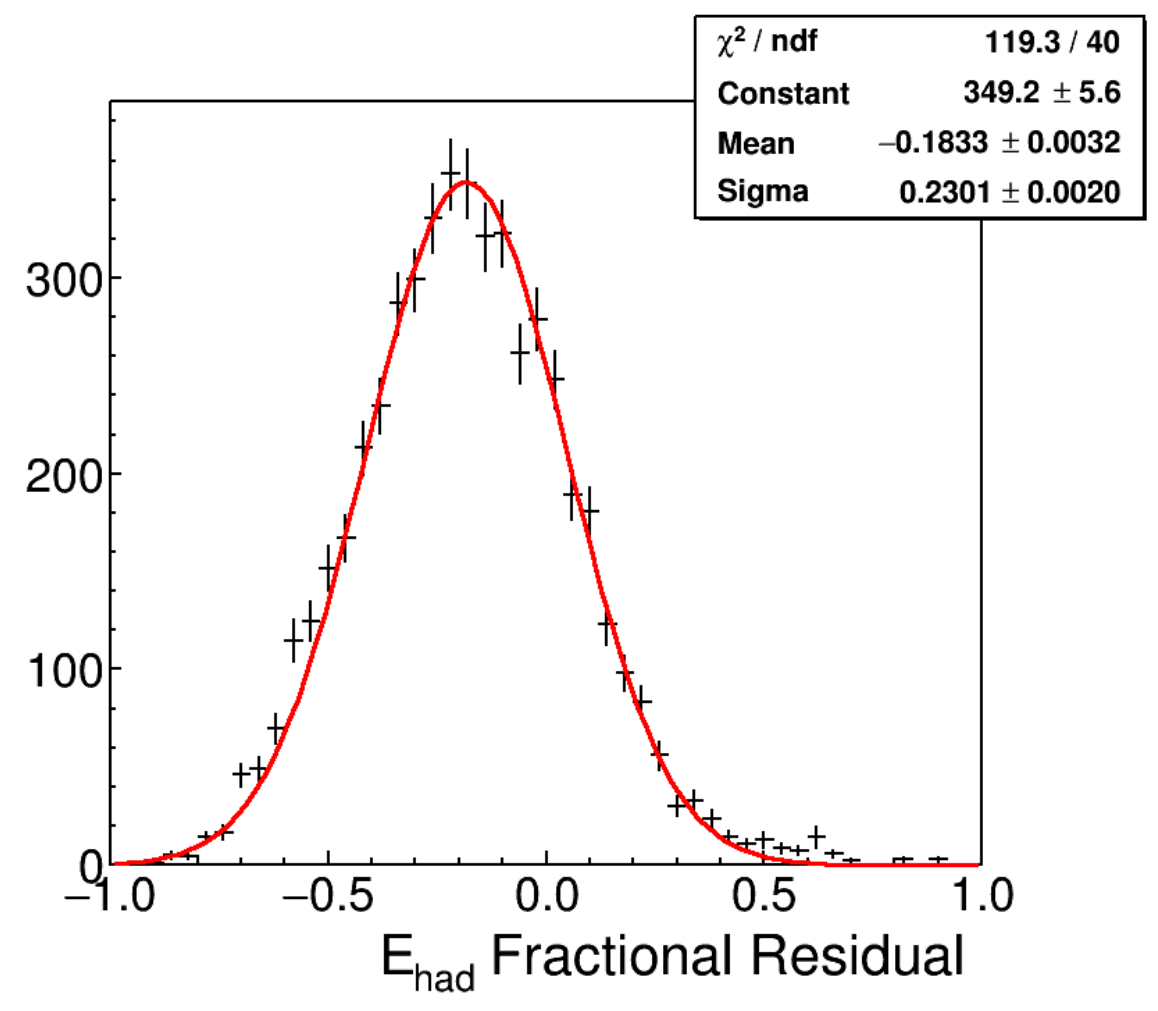

Figure 6.1: Hadronic energy fractional residual: $\left(E_{\text {had }}\right.$ reconstructed $-E_{\text {had }}$ true $) / E_{\text {had }}$ true.

\subsection{EVENT SELECTION}

The purpose of this first stage of the cross section calculation is to select all and only the events from the data that would satisfy the signal definition. It is furthermore important 
that selected events are well-reconstructed. Several event selection cuts were studied and implemented in order to optimize the signal efficiency and purity, to optimize the event kinematic reconstruction, and to minimize the systematic errors where possible.

The full list of cuts includes:

- Fiducial volume interaction vertex The interaction vertex is required to be within the tracker region between modules 27 and 81 with apothem $850 \mathrm{~mm}$. N.b. this cut is tighter than than the fiducial volume defined in the signal definition. See discussion below.

- MINOS matched muon A single muon track is required, defined as a long track, anchored at the interaction vertex, exiting the back of the MINERvA detector, and having negative reconstructed charge.

- Isolated clusters At most one isolated cluster of energy is allowed.

- Hadron Track At least one non-muon, non-exiting track connected to the interaction vertex is required.

- Michel electron At least one michel electron candidate must be matched to the endpoint of a hadron track, hereafter referred to as a pion candidate.

- Pion candidate track — particle ID score The pion candidate track must be pionlike according to the log-likelihood ratio score.

- Pion candidate track — node energy The energy of terminal six nodes of the pion candidate track must be consistent with a stopping pion.

- Pion Multiplicity Exactly one pion candidate track must pass the michel, PID, and node cuts.

- Invariant hadronic mass the event's reconstructed invariant hadronic mass, $W_{\text {exp }}$, is required to be less than $1.4 \mathrm{GeV}$.

These cuts can be divided into five categories: fiducial volume interaction vertex selection, muon selection, charged pion selection, neutral pion veto, and kinematic constraints. They will be discussed in the following sections. 


\subsubsection{Fiducial interaction vertex selection}

The neutrino interaction vertex is required to fall within the fiducial volume in order to ensure an interaction on scintillator, and not on the upstream targets, downstream ECAL lead, or outer-detector (apothem $900 \mathrm{~mm}$ ) ECAL lead. The cut spans from module 27 (z-position $5990 \mathrm{~mm}$ ) to module 79 (z-position $8340 \mathrm{~mm}$ ) with $850 \mathrm{~mm}$ apothem.

Note that the cut on the fiducial volume is tighter than the fiducial volume defined by the signal criteria. This means that events with reconstructed vertex inside the cut fiducial volume and truth vertex outside the cut fiducial volume are not subtracted as background, but are instead they are corrected for during the unfolding stage. Similarly, events with reconstructed and truth vertex outside the cut fiducial volume but inside the signal fiducial volume are replaced at the efficiency-correction stage. The model dependence introduced by this mismatch is merely due to position-translation, and it is a carefully chosen lesserevil compared to the alternative in which model dependence is injected by the modeling of non-scintillator backgrounds.

In the case that a time slice contains multiple vertices, at most one vertex and interaction is considered. Vertex priority is given according to the following conditions, in order:

1. prefer the interaction with a muon

2. prefer the interaction with the most short tracks

3. prefer the most upstream interaction

In the LE, multi-vertex slices occurred at a rate of $<1 \%$. In the ME, with the increased NuMI beam intensity and an offsetting adjustment to the time-slicing algorithm, the rate is now at the several-percent level.

\subsubsection{Muon selection}

A muon is identified as a long track that exits the back of the MINERvA detector. This analysis requires a muon track to be matched to MINOS and reconstructed with a negative

charge. This requirement selects charged current events with $99 \%$ purity, with a rare neutral current, $\pi \rightarrow \mu$ background. Muons without a match are cut because the charge is needed 
to identify the event as $\nu_{\mu}$ or $\bar{\nu}_{\mu}$, and because a momentum measurement can only be made with the MINOS track. Muons that stop in MINERvA are not considered, both because they lack a charge measurement, and because they are difficult to distinguish from pions.

While the NuMI beam is in FHC mode, the MINOS magnetic field is aligned so that negatively charged muons (originating from $\nu_{\mu} \mathrm{CC}$ interactions) are focused, giving a longer track in MINOS, providing the best momentum and charge measurement possible.

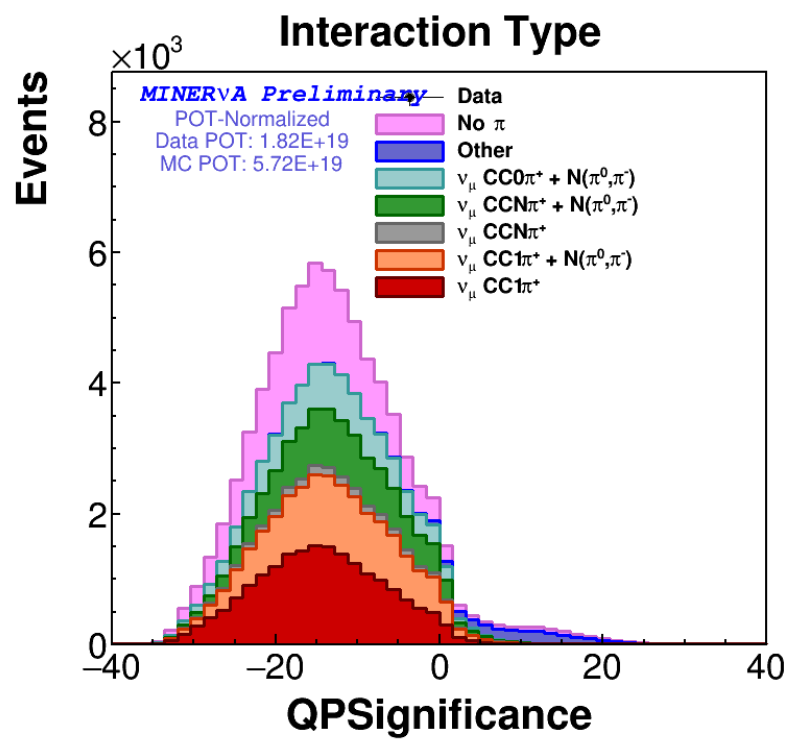

Figure 6.2: Non-CC $\nu_{\mu}$ events fall above 0 in the significance metric, charge/momentum.

\subsubsection{Charged pion selection}

To select events with charged pions, a michel electron is first matched to non-muon track endpoints, promoting them to "pion candidates". The pion candidates are then subject to a particle identification score cut and a stopping-pion terminal node energy cut.

6.3.3.1 Michel electron match Among the non-muon tracks anchored to the interaction vertex, positively-charged pion tracks are identified by the presence of a delayed-time "michel" electron within the vicinity of the track endpoint. The detection of a michel electron is a very pure signature of a charged pion. A michel electron is the product of a muon 
decay, by way of a stopped $(\mathrm{KE}=0)$ pion decay. The pion decays into a muon with lifetime $\tau \sim 26 \mathrm{~ns}$ and branching fraction $\sim 0.99999$. The muon subsequently decays into an electron and three neutrinos with $\tau \sim 2.2 \mu s$ and branching fraction $\sim 1$.

To find michels and match them to tracks, first clusters of hits are selected as michel candidates. Michel candidates are chosen by searching for hit clusters (i) occuring in time slices after the neutrino interaction, (ii) occuring within a radius of $50 \mathrm{~cm}$ from the interaction vertex, and (iii) having total energy consistent with an electron from the three-body decay of a muon.

Next the distance of each michel candidate to each track endpoint is measured. The separation distance between the michel candidate and track endpoint is determined by the category that each michel candidate falls into:

1. Fitted michel With hits across multiple planes, the michel candidate energy cluster is fit to a straight line segment, and the distance to the track endpoint is measured from the segment edge closest to the endpoint.

2. Unfitted michels Michels with hits that cannot be fit to a line but which span at least two planes are unfitted michels. The distance to the track endpoint is measured from an energy-averaged mean of the cluster.

3. One-view michels With energy deposited in just one of the $\mathrm{X}$, U, or V planes, these contain the minimum qualifications for a michel candidate. As with unfitted michels, the distance to the track endpoint is measured from an energy-averaged mean of the cluster.

Next, michel-endpoint matches are formed provided the separation distances are smaller than a maximum value, depending on michel categorization. The cut values are given in table 6.1 .

With separation distances above these values a match between michel candidate and track endpoint fails. In situations where a michel matches to multiple endpoints, the match with the shorter separation distance is preferred. With typical separation distances of 5-10 $\mathrm{cm}$, fitted michels more accurately identify the correct track endpoint. These distances were optimized to minimize the non-pion background fraction. While the michel cut increases the signal purity by $22.5 \%$, it reduces the efficiency significantly, from $24 \%$ to $3.5 \%$. The 
Table 6.1: Michel fit category and corresponding maximum separation cut value

\begin{tabular}{l|c} 
Category & Maximum Separation $(\mathrm{cm})$ \\
\hline Fitted & 7.5 \\
Unfitted & 50 \\
One View & 50
\end{tabular}

primary reason for this low cut efficiency is that only stopping pions, i.e. pions that lose all of their energy through ionization and atomic excitation before stopping or decaying, reliably produce a michel. Many pions decay or interact inelastically with a detector nucleus before losing all of their energy. The michel efficiency is also low because michels have a low amount of energy ( $55 \mathrm{MeV} \max$ ) and do not always convert before ranging out [61].

A successful match between a michel and track endpoint promote the matched track to a pion candidate. 


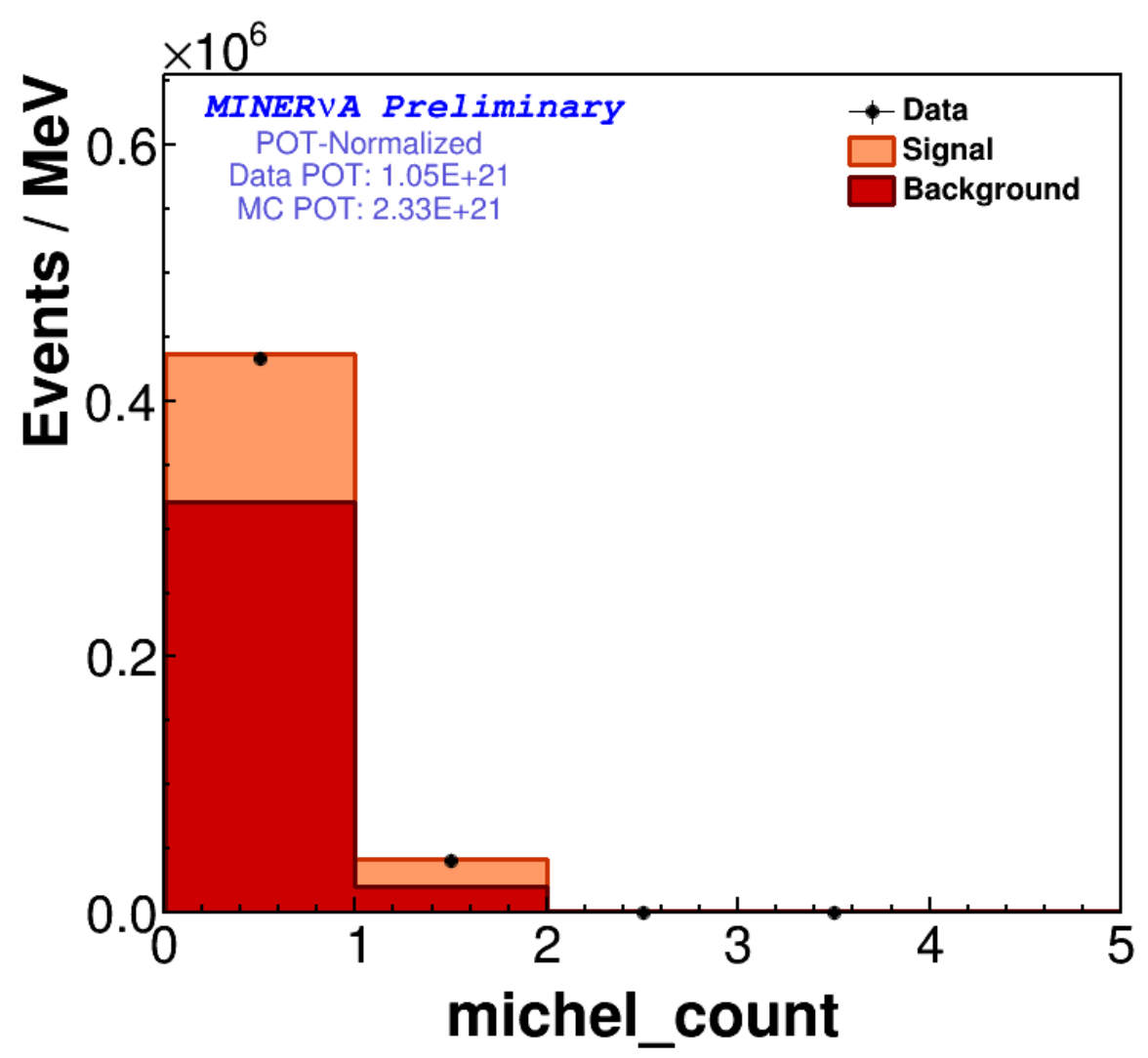

Figure 6.3: Number of matched michels by signal and background showing the effectiveness of the michel cut.

6.3.3.2 Track particle identification (PID) score After michel matches promote tracks to pion candidates, each track is subjected to a particle identification (PID) score cut. The primary purpose of the PID cut is to remove protons that have erroneously passed the michel cut and been promoted to pion candidate. A PID score is assigned to each track and identifies the track as more pion-like or more proton-like. The score leverages the fact that, according the Bethe formula describing the passage of charged particles through matter[121], each particle deposits its own characteristic energy-loss, $\frac{d E}{d x}$ profile in the detector. The $d E / d x$ profiles for a simulated pion and proton particles in MINERvA, compared with their Bethe curves are shown in 6.4. Side-by-side proton and pion tracks in MINERvA are shown 
in Figure 5.3.

To construct the track score, first a likelihood function was formed for each node of a pion track and a proton track from a large set of simulated pion and proton particle gun Monte Carlo. The likelihoods are multiplied across all nodes, to obtain the likelihood that a test track with node energies $E$ is of identity $a$ given the node energies for the entire track:

$$
\mathcal{L}(a \mid \text { track })=\Pi_{\text {nodes }} P\left(E_{\text {node }} \mid a\right)
$$

And the test track's PID score is the log of the ratio of the pion and proton likelihoods:

$$
\text { PID Score }=\sum_{\text {nodes }}\left(\log P\left(E_{\text {node }} \mid \pi\right)-\log P\left(E_{\text {node }} \mid p\right)\right)
$$

By the Neyman-Pearson lemma, the log ratio of the likelihoods is the most powerful statistical test [95].

It was found that overlapping tracks near the interaction vertex can obscure the node energies and bias the likelihood score. For this reason, when the node energy falls steeply (more than $1.9737 \mathrm{MeV}$ ) between two nodes in the first 10 nodes of the track, then the high-energy node is removed from score calculation.

When the pion or proton interacts in the detector, the observed terminal node corresponds to node $n$ of the likelihood template. To account for this, the likelihood is calculated at all possible $n$ offsets, and the maximum likelihood is used.

The PID score is required to be greater than 0. If a pion candidate fails this condition, it is demoted from pion candidate status. If the number of pion candidates drops to zero, the event fails the event selection. The PID cut has a $64 \%$ cut efficiency and it increases the purity by $5 \%$, though its effect on signal and purity do not tell the full story. The PID cut is effective at removing protons that have been mis-identified as pion candidates. Thus, in addition to the gain in purity, the PID cut also improves the pion kinematic reconstruction by ensuring that protons are not reconstructed with a pion hypothesis. 


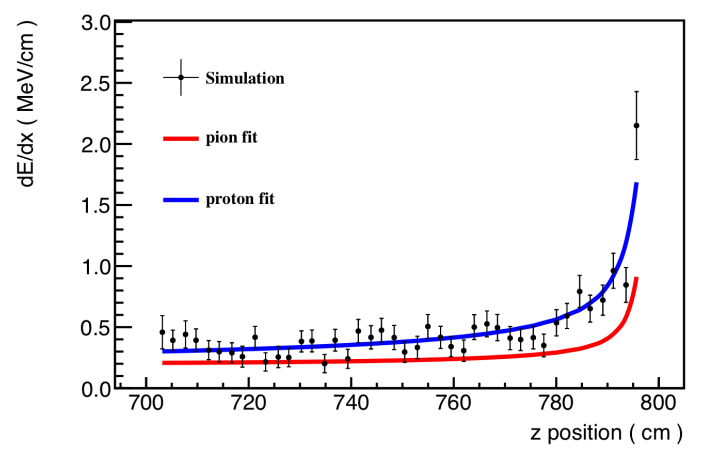

(a)

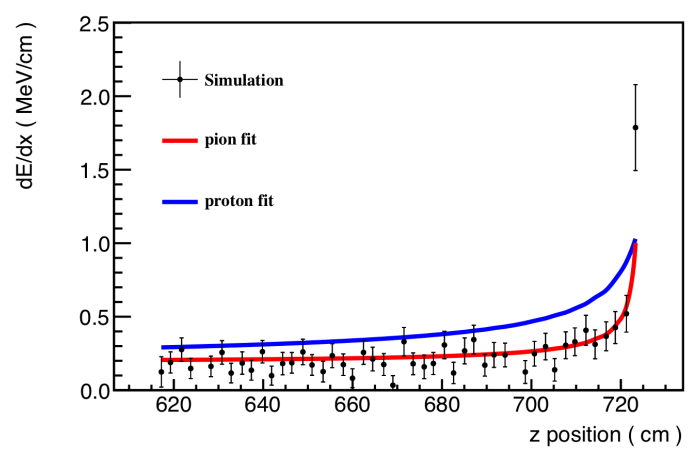

(b)

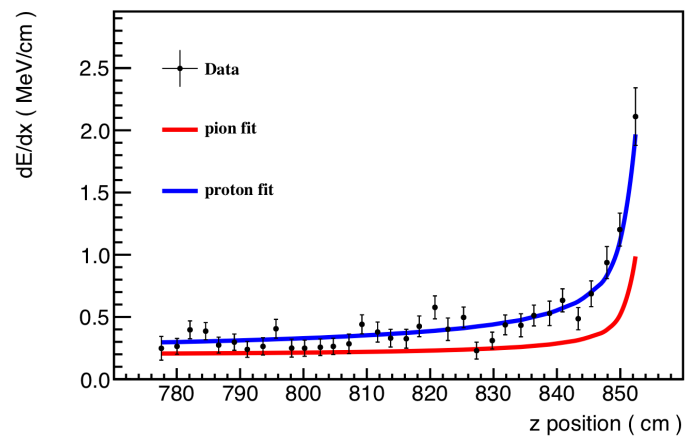

(c)

Figure 6.4: Energy loss profiles for a MINERvA-simulated proton (top) and pion (middle) compared to Bethe curves. And a MINERvA proton candidate (bottom). Figures from [20]. 


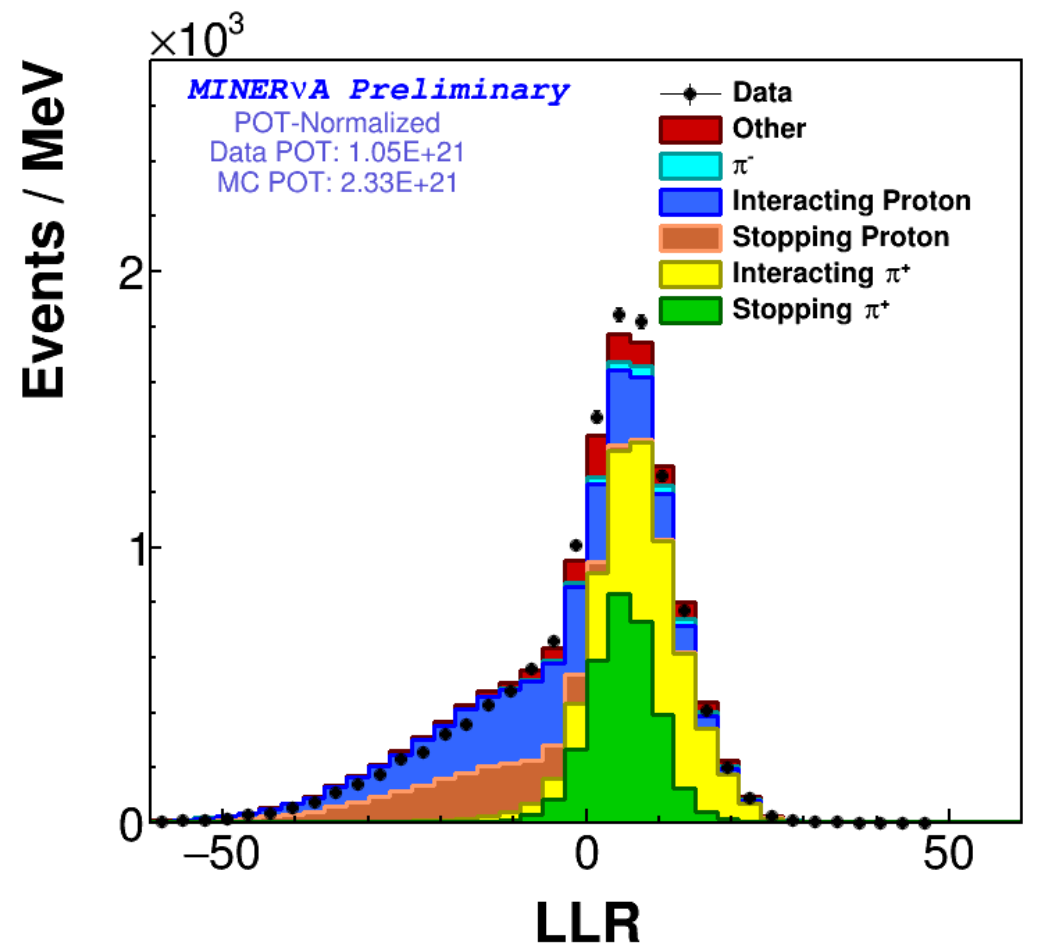

Figure 6.5: The log-likelihood ratio particle identification score separates pions and protons well. 
6.3.3.3 Track terminal node energy After michel matches and the PID cut, remaining pion candidates are subjected to a node energy cut. Like the PID cut, the node cut is useful for removing protons that have been confused for pions, but it is also effective at separating inelastically interacting pions - which typically have bad energy reconstruction - from pions that range out their kinetic energy.

In both cases - proton and inelastic track removal - the distinct endpoint energy loss profiles $\frac{d E}{d x}$ of the pions and protons is used. When pions or protons lose all their kinetic energy and come to a stop in the detector, their $\frac{d E}{d x}$ shows a sharp increase (called a Bragg peak) at the end of the track.

The absence of such a peak indicates that the particle, either a pion or a proton, may have interacted. Thus interacting particles can be removed with a cut on the lower end of the candidate track's node energy spectrum.

The precise Bragg peak shape is different for pions and protons, with protons having the steeper energy loss. Thus protons can be removed with a cut on the upper end of the candidate track's node energy spectrum.

The cut is performed by considering the energies in the terminal six nodes of pion candidate tracks. The cut values are given in Table 6.2.

Table 6.2: Track terminal node energy cut values by node

\begin{tabular}{l|c|c} 
Node & Minimum Energy $(\mathrm{MeV})$ & Maximum Energy $(\mathrm{MeV})$ \\
\hline terminal-0 + (terminal-1) & $6 \mathrm{MeV}$ & $32 \mathrm{MeV}$ \\
terminal-2 & $2 \mathrm{MeV}$ & $22 \mathrm{MeV}$ \\
terminal-3 & $0 \mathrm{MeV}$ & $19 \mathrm{MeV}$ \\
terminal-4 & $0 \mathrm{MeV}$ & $31 \mathrm{MeV}$ \\
terminal-5 & $0 \mathrm{MeV}$ & $60 \mathrm{MeV}$
\end{tabular}

Pion candidates who fail the node cut lose their candidate status, and as with the PID cut, if the number of pion candidates in an event drops to zero, the event fails the event selection. The node cut has a $76.5 \%$ cut efficiency and increases the signal purity by $6.3 \%$. 


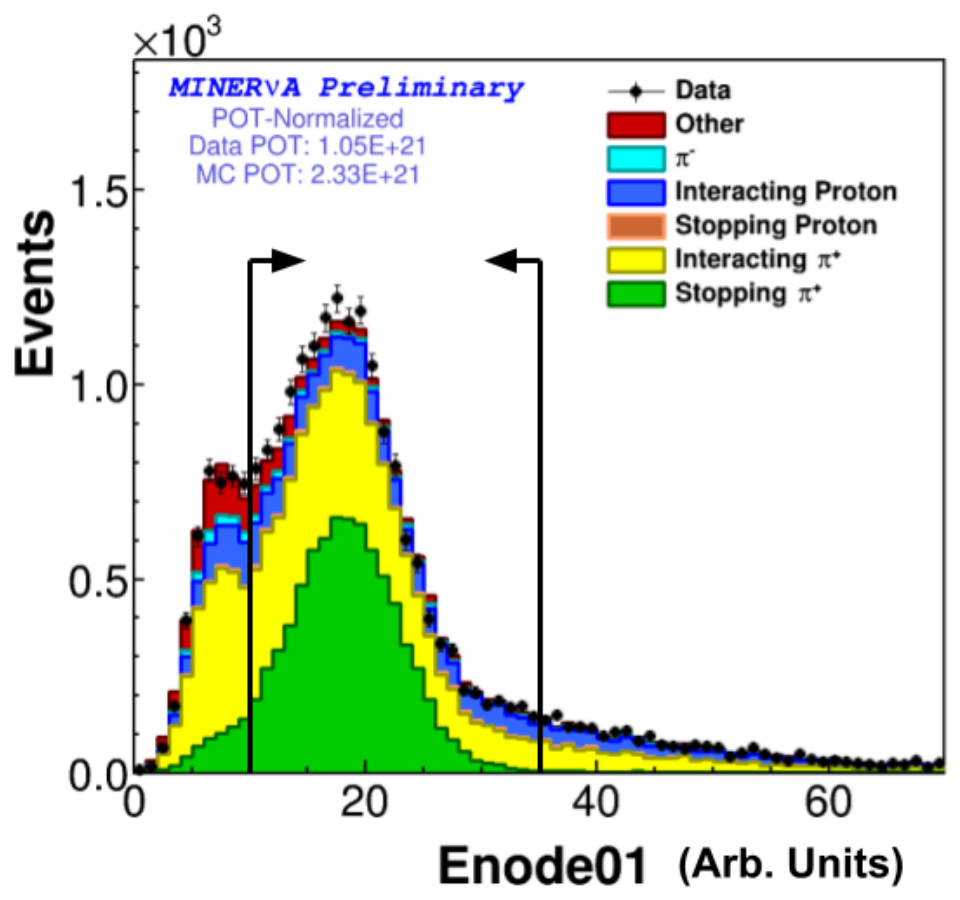

Figure 6.6: The sum of the energy of the terminal and terminal +1 nodes for candidate pion tracks. Interacting particles and protons pile up at the upper and lower edges.

\subsubsection{Neutral pion veto}

The characteristic signatures of a neutral pion in MINERvA are its two decay-photon electromagnetic showers (see Figure 5.3). Neutral pion events can be removed by allowing at most one isolated energy cluster in the event. The isolated energy clusters are deposits that have not been associated with any other object in the event and that fall within $\sim 30$ ns of the interaction. In addition to removing neutral pions, isolated energy deposits are correlated with high- $W$ background events, and the cut helps remove these. The isolated energy cut has a $91.9 \%$ cut efficiency and increases the signal purity by $9.4 \%$. 


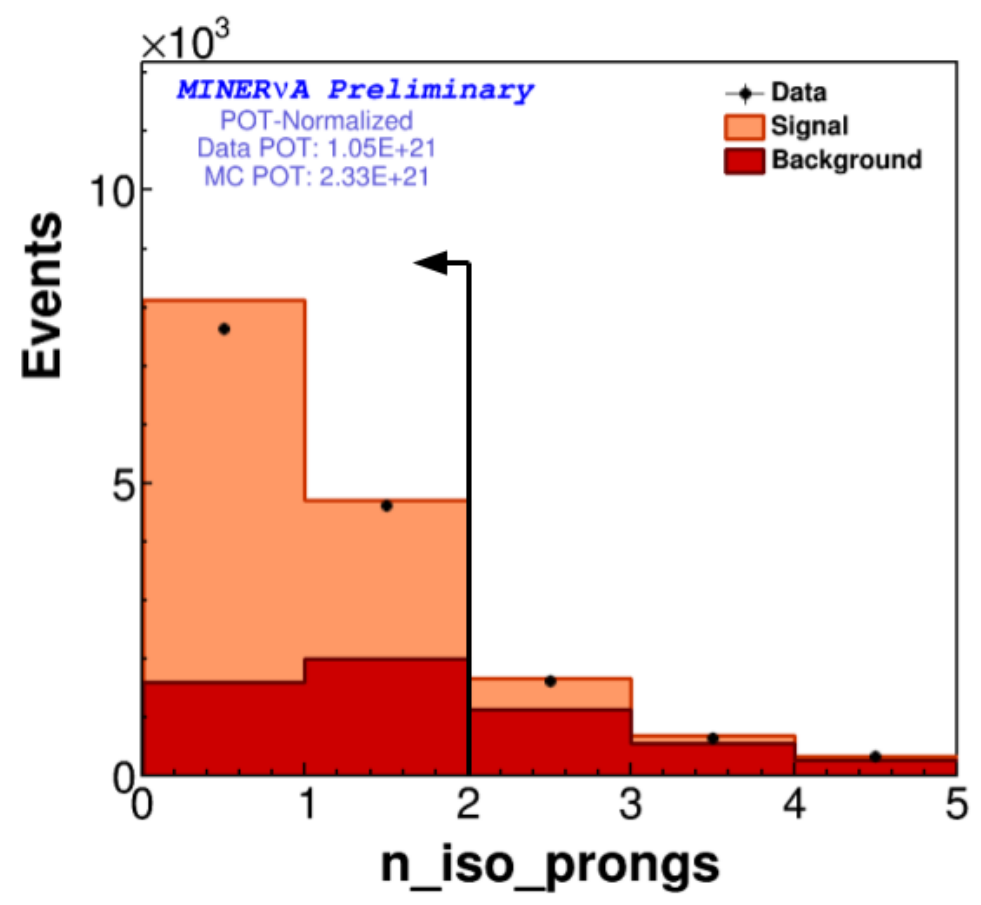

Figure 6.7: Number of isolated energy deposits (providing an effective $\pi^{0}$ removal cut), broken down into simulated signal and background categories.

\subsubsection{Kinematic cuts}

The only kinematic cut applied is on the experimental invariant hadronic mass $W_{\text {exp }}$, as defined in Equation 6.4.

Inefficiencies $(\sim 25 \%)$ and relatively small purity gains $(\sim 20 \%)$ are introduced into the $W_{\text {exp }}<1.4$ cut by poor $E_{\text {had }}$ reconstruction. High- $W$ events are characterized by more track overlap and more neutral energy making, the events harder to reconstruct. The $\sim$ $25 \%$ of signal events lost by the cut are due to reconstruction "feed up", in which events are reconstructed with more hadronic energy compared to truth. More commonly, high- $W$ events "feed down" into the sample contaminating an otherwise pure cut. 


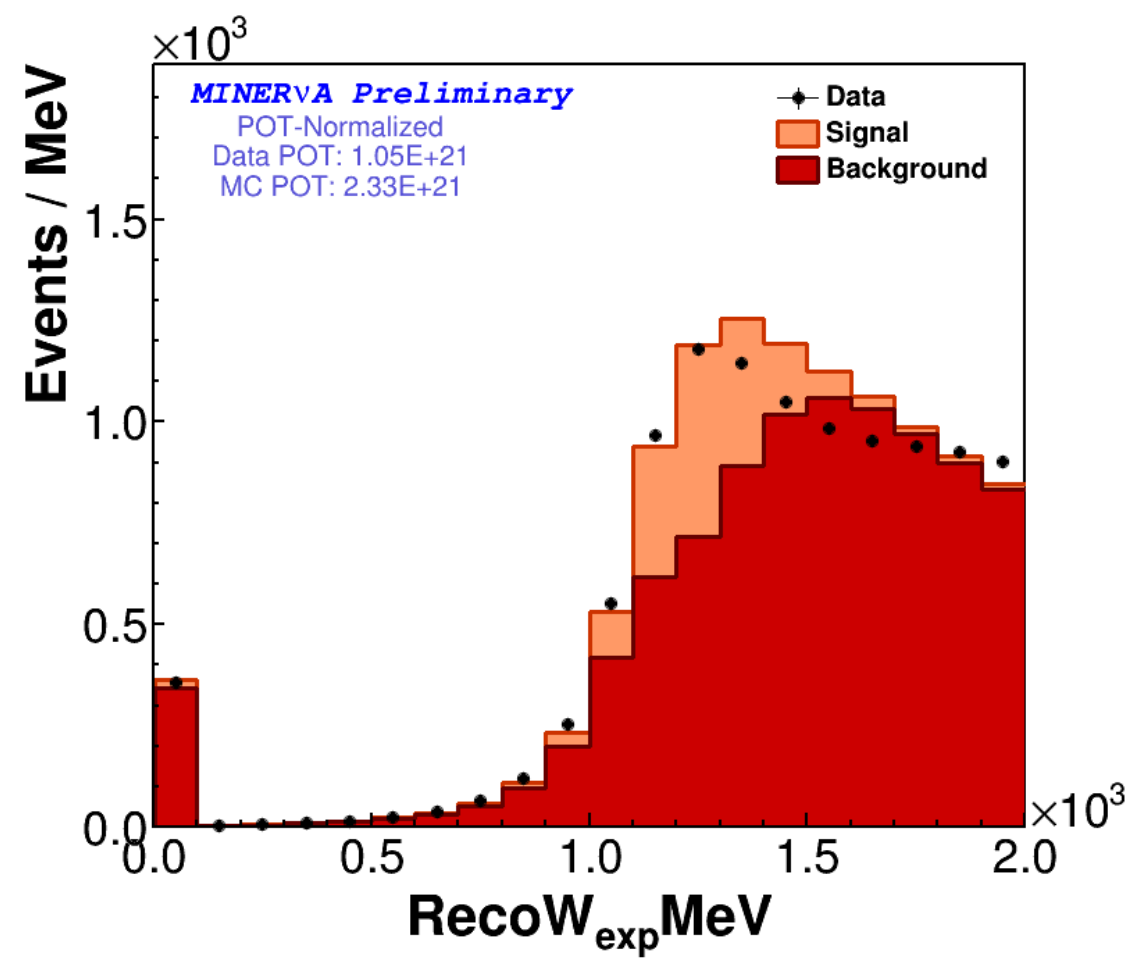

Figure 6.8: $W_{\exp }$ distribution, before the $W_{\text {exp }}$ cut, broken down by signal and background.

No explicit cut is made on the pion lower and upper kinetic energy limits. As explained in Section 6.2, the lower and upper detection thresholds are $\sim 35 \mathrm{MeV}$ and $350 \mathrm{MeV}$.

\subsubsection{Alignment of signal definition, detector acceptance, and event selection cuts}

When a signal definition defines the signal process phase space to be wider than the detector is capable of measuring or wider than event selection cuts, model dependence is injected into the measurement when the model fills-in events during unfolding or efficiency correction.

In this respect, there are two known mismatches in this analysis, where the detector acceptance and cuts do not closely match the signal definition.

The first concerns pion angle. Particles that emerge from the interaction vertex at between 70 and $110^{\circ}$ with respect to the $\mathrm{z}$-axis have poor detection efficiency, particle identifi- 
cation, and kinematic reconstruction resolution. Adding such a signal constraint was outside the scope of this analysis timeline.

The second concerns muon momentum. Muons have a low-momentum detection threshold that is not reflected in the signal definition. MINOS's lower tracking threshold for muons is $150 \mathrm{MeV}$, which implies a 1-1.5 GeV lower limit on incident momentum in MINERvA, where the difference accounts for energy loss through MINERvA and traveling the additional $2 \mathrm{~m}$ from the back of MINERvA to the front of MINOS. Currently the efficiency correction repopulates the sample with low-momentum muon events. A signal event criterion restricting low-energy muons should be considered for a future measurement.

\subsubsection{Event selection results}

Figure 6.9 is a table showing the number of signal, background, total MC, and data events remaining in the sample after each cut. The cut efficiency, net signal efficiency, and net signal purity are also included. "N MC (scale)" is the amount of MC scaled to match the data POT. After all cuts, simulation predicts 20392 signal events and 7943 background events remain in the sample. 12799 data events are selected.

Figure 6.10 shows the reconstructed distributions of the seven analysis variables for events passing the analysis selection. The error bars on the simulation reflect the comparable uncertainties in detector measurement, the flux measurement, and the neutrino-nucleon cross sections predicted by GENIE. 
DataPOT: $1.05085 \mathrm{e}+21$. MCPOT: $2.32639 \mathrm{e}+21$.

\begin{tabular}{|l|l|l|l|l|l|l|l|l|l|l|l}
\hline & \multicolumn{4}{|c|}{ Signal } & \multicolumn{2}{|c|}{ Background } & \multicolumn{3}{c|}{ Total } & \multicolumn{2}{c}{ Data } \\
\hline & $\mathrm{N}$ & Eff & Cut Eff & Pur & $\mathrm{N}$ & Eff & N & Eff & N MC (scale) & N Data & $\begin{array}{l}\text { Data' } \\
\text { MC }\end{array}$ \\
\hline No Cuts & 1269548 & $100.0 \%$ & $100.0 \%$ & $1.1 \%$ & 115318091.8 & $100.0 \%$ & 116587640.1 & $100.0 \%$ & NA & NA & NA \\
\hline Anatool Precuts & 1083026 & $85.3 \%$ & $85.3 \%$ & $4.9 \%$ & 21169545.2 & $18.4 \%$ & 22252571.2 & $19.1 \%$ & NA & NA & NA \\
\hline MINOS Muon & 841596 & $66.3 \%$ & $77.7 \%$ & $9.4 \%$ & 8156065.5 & $7.1 \%$ & 8997661.8 & $7.7 \%$ & 4064306.9 & 4145774.0 & 1.0 \\
\hline$>=1$ Hadron Track & 406624 & $32.0 \%$ & $48.3 \%$ & $9.0 \%$ & 4131791.5 & $3.6 \%$ & 4538415.2 & $3.9 \%$ & 2050033.9 & 2037931.0 & 1.0 \\
\hline$W_{\text {experimental }}$ & 303304 & $23.9 \%$ & $74.6 \%$ & $28.7 \%$ & 755287.5 & $0.7 \%$ & 1058591.5 & $0.9 \%$ & 478173.2 & 474424.0 & 1.0 \\
\hline$>=1$ Michel & 44953 & $3.5 \%$ & $14.8 \%$ & $51.2 \%$ & 42871.5 & $0.0 \%$ & 87824.9 & $0.1 \%$ & 39671.1 & 38821.0 & 1.0 \\
\hline LLR PID & 29011 & $2.3 \%$ & $64.5 \%$ & $56.2 \%$ & 22626.4 & $0.0 \%$ & 51637.7 & $0.0 \%$ & 23325.1 & 23651.0 & 1.0 \\
\hline Node & 22192 & $1.7 \%$ & $76.5 \%$ & $62.5 \%$ & 13332.1 & $0.0 \%$ & 35523.9 & $0.0 \%$ & 16046.4 & 15356.0 & 1.0 \\
\hline$<2$ Isolated Prongs & 20400 & $1.6 \%$ & $91.9 \%$ & $71.9 \%$ & 7970.6 & $0.0 \%$ & 28371.0 & $0.0 \%$ & 12815.4 & 12254.0 & 1.0 \\
\hline Pion Multiplicity & 20392 & $1.6 \%$ & $100.0 \%$ & $72.0 \%$ & 7942.6 & $0.0 \%$ & 28334.8 & $0.0 \%$ & 12799.0 & 12246.0 & 1.0 \\
\hline
\end{tabular}

Figure 6.9: Event selection table by cut. 


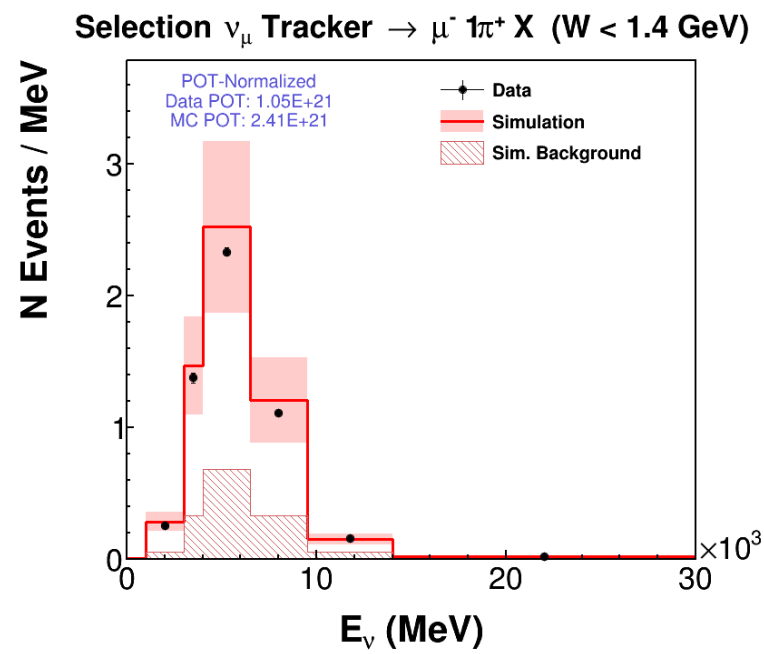

(a) $E_{\nu}$ event selection

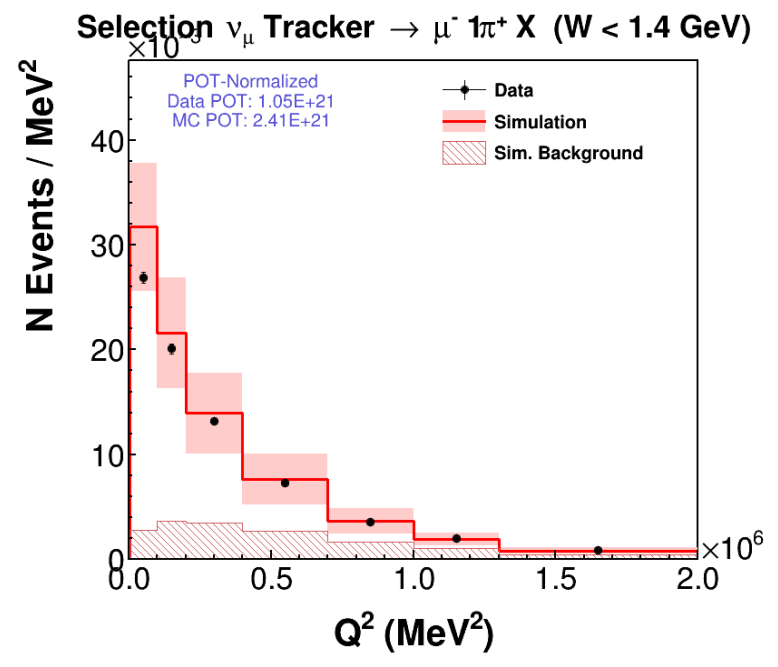

(b) $Q^{2}$ event selection 


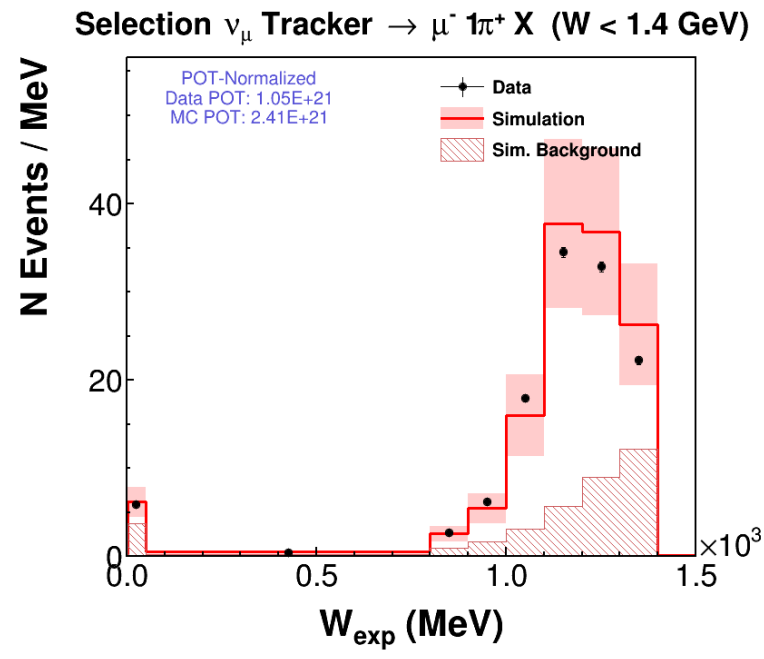

(c) $W_{\text {exp }}$ event selection

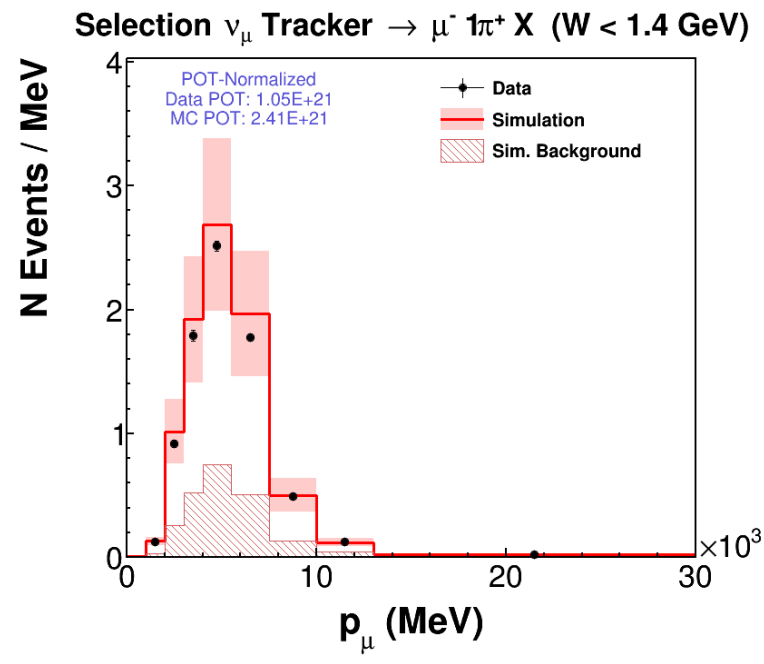

(d) $P_{\mu}$ event selection 


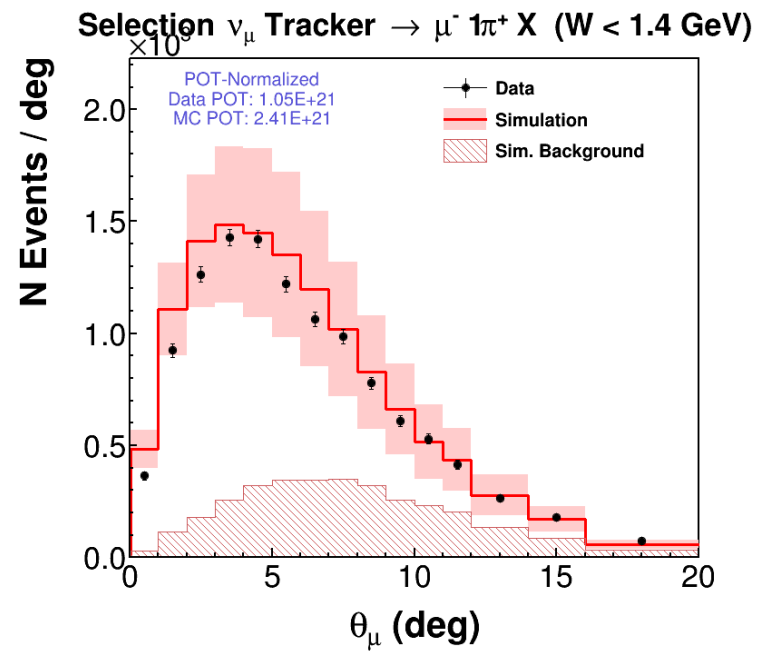

(e) $\theta_{\mu}$ event selection

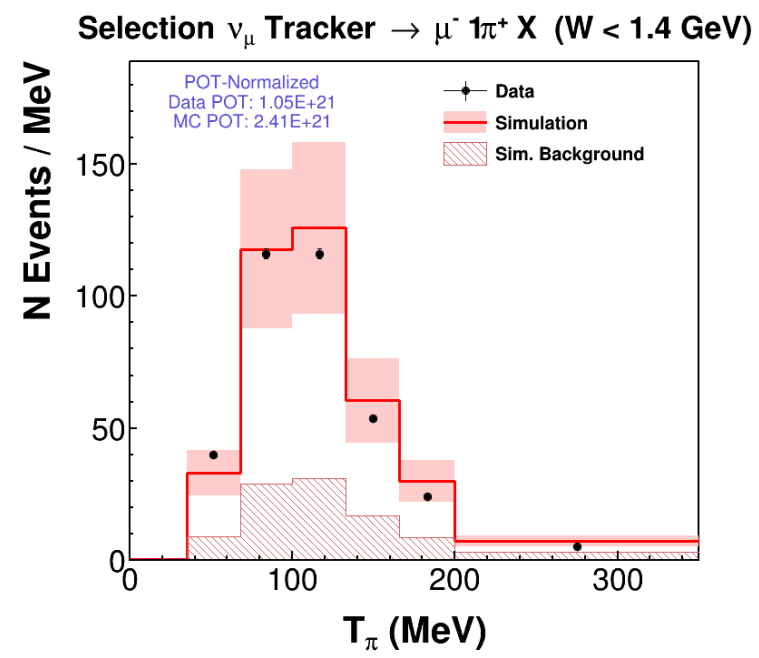

(f) $T_{\pi}$ event selection 


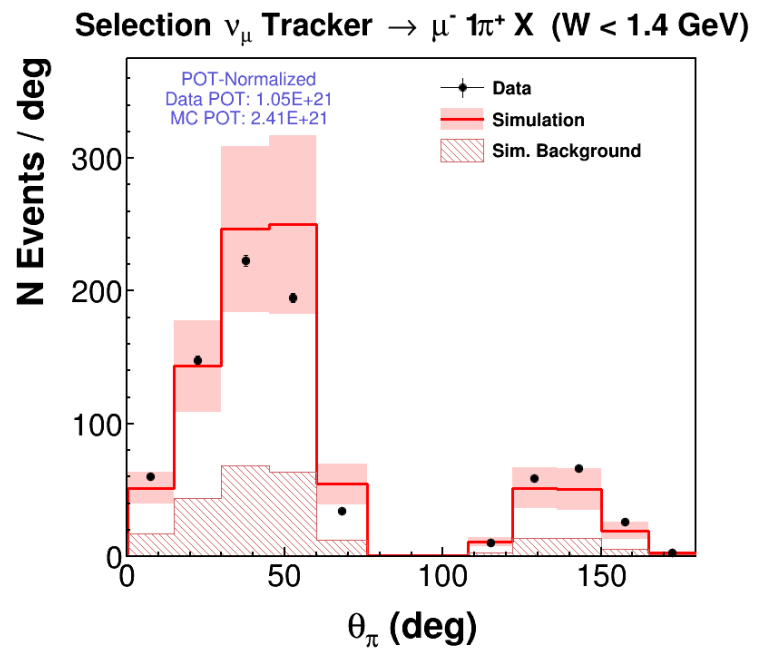

(g) $\theta_{\pi}$ event selection

Figure 6.10: Event selection distributions for all analysis variables. 


\subsection{BACKGROUND SUBTRACTION}

The background events remaining in the selected sample after cuts is predicted by simulation.

Figure 6.11 shows the background events as a function of $Q^{2}$ broken down by interaction channel. The michel cut is very effective at removing quasi-elastic events which produce no pion. A large non-resonant background remains, primarily from DIS $W_{\text {exp }}>1.4 \mathrm{GeV}$ events. The resonant background constsists of events that fail kinematic cuts, such as high- $W_{\text {exp }}$ or $T_{\pi}$ above or below threshold.

Before outright subtraction of the background from the selection, the simulated background is scaled to agree with a sample of in situ experimental data that is strictly independent, but kinematically adjacent to the signal region. This step, called background tuning, reduces the cross section calculation's dependence on the model by reducing the systematic uncertainties on the background prediction.

\section{Background}

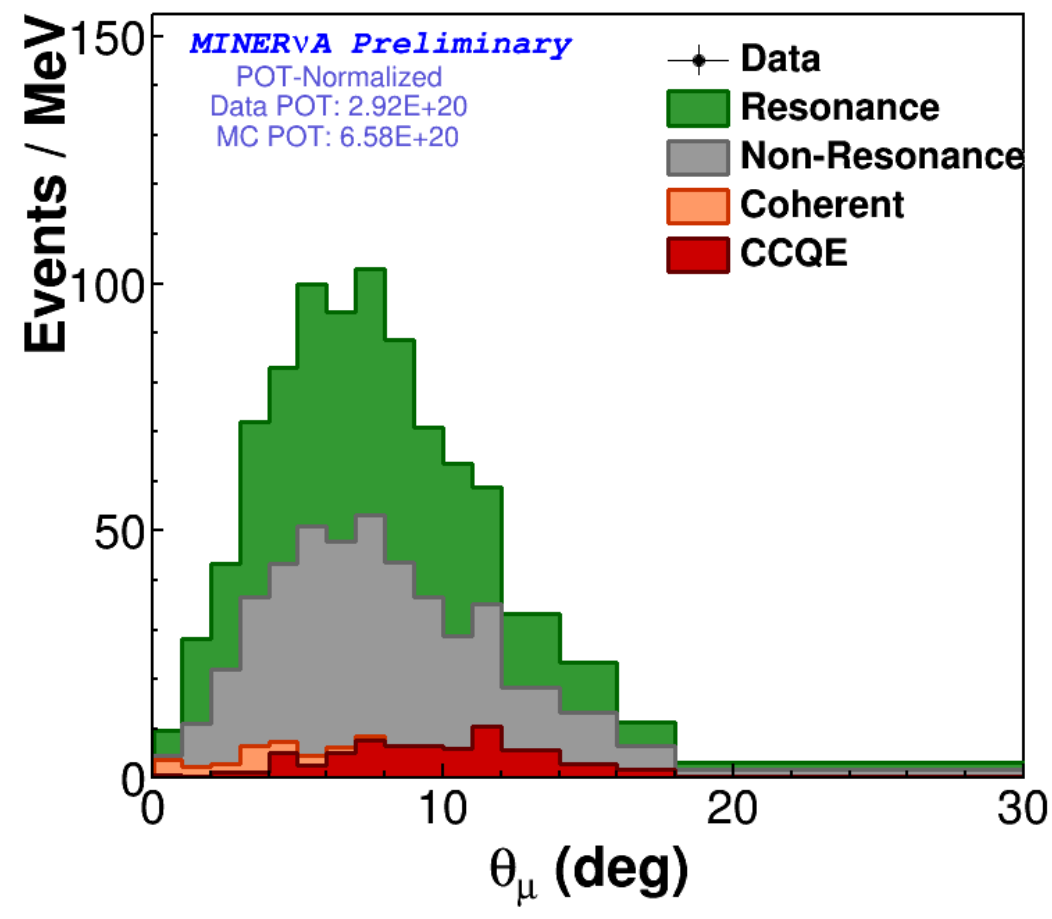

Figure 6.11: Background sample as a function of $Q^{2}$, broken down into interaction channels. 
The sideband sample is a background-dominated region filled with events that pass all event selection cuts except for the $W_{\text {exp }}$ cut and furthermore have $W_{\exp }>1.5 \mathrm{GeV}$. The constraint is performed by scaling the simulated sideband distribution to the experimental sideband data.

The constraint is performed using an iterative $\chi^{2}$ minimization technique

$$
\chi^{2}=\sum_{i}^{\mathrm{N} \text { bins }} \frac{\left(N_{\text {Simulation }, i}-N_{\text {Data }, i}\right)^{2}}{\left(\sigma_{\text {Simulation }, i}^{2}+\sigma_{\text {Data }, i}^{2}\right)}
$$

where the simulated sideband $N_{\text {Sideband }, i} \equiv N_{\text {Total Simulation }, i}-N_{\text {Signal }, i}$ is floated to minimize $\chi^{2}$

More precisely, $N_{\text {Total Simulation, } i}$ is subdivided into three components, defined by regions of true simulated $W_{\text {exp }}$, and the normalizations of each region are fit simultaneously.

The three regions are low $\left(W_{\text {exp }}<1.4 \mathrm{GeV}\right)$, middle $\left(1.4 \mathrm{GeV}<W_{\text {exp }}<1.8 \mathrm{GeV}\right)$, and high $\left(W_{\exp }>1.8 \mathrm{GeV}\right)$. The boundary of $1.8 \mathrm{GeV}$ was chosen as the location where the underlying generator model switches from a resonance + hadronization (KNO + PYTHIA) model to a pure hadronization (PYTHIA) model[27]. The results of the fit pull the low, middle, and high regions by factors of $1.0,0.87$, and 0.99 , respectively, with a $\chi^{2} / n d f$ of 1.18 . This approach assumes that the distribution for $N_{i}$ is Gaussian. A Poisson approach[28] was also considered, but the differences in results were small.

After applying the scale factors to the background in the signal region, the background is subtracted bin-by-bin. It is in this step of the cross section calculation that systematic uncertainties are added to the data points, and thus the error bars on the backgroundsubtracted data points contain systematic and statistical errors, while only statistical errors are left on the simulated distribution. 


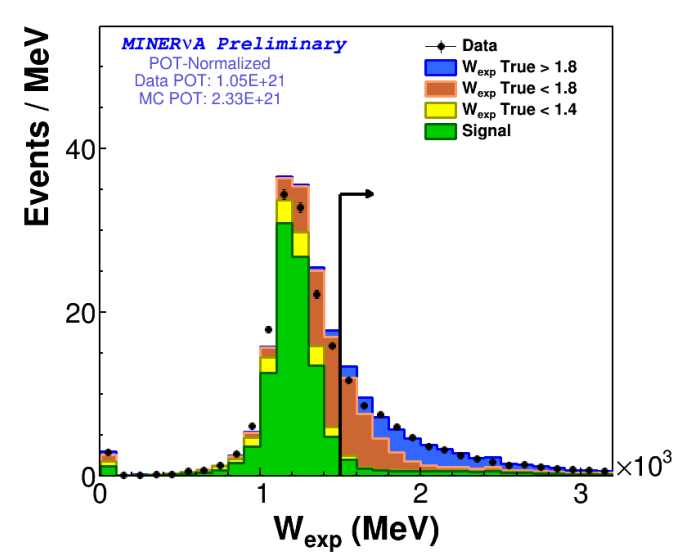

(a) The region to the right of the arrow shows the sideband region. The normalization of each background sub-region, broken down by true $W$, is floated to fit the data.

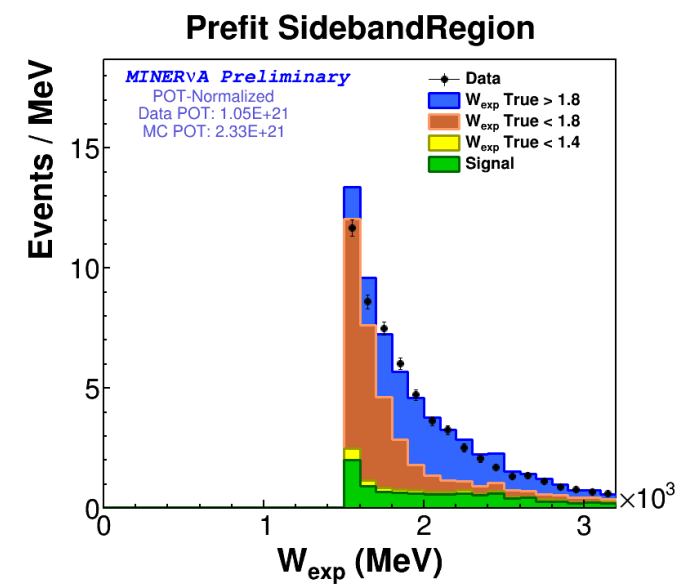

(b) Sideband sample plotted in $W_{\text {exp }}$ before fit.

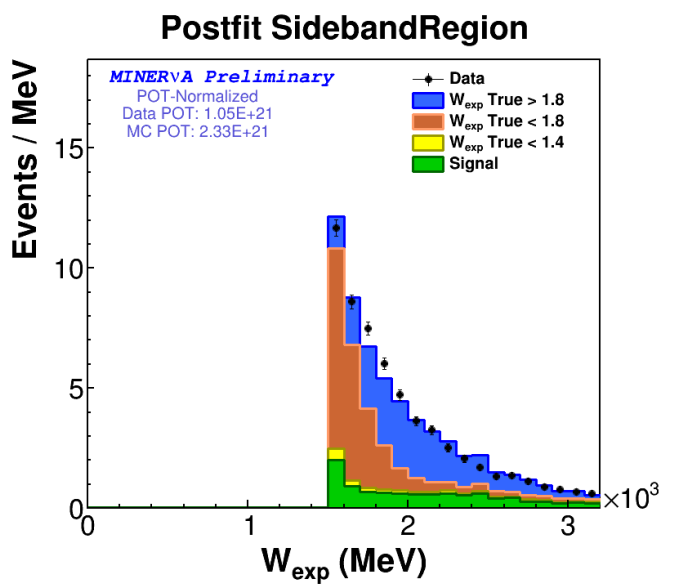

(c) Sideband sample plotted in $W_{\text {exp }}$ after fit.

Figure 6.12: Signal and sideband regions, pre- and post-fit. 


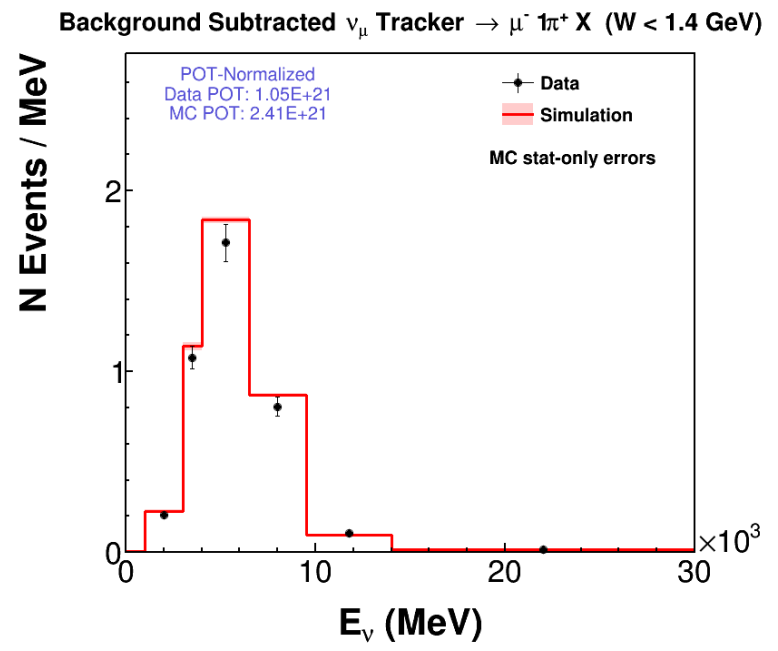

(a) $E_{\nu}$ background subtracted event selection

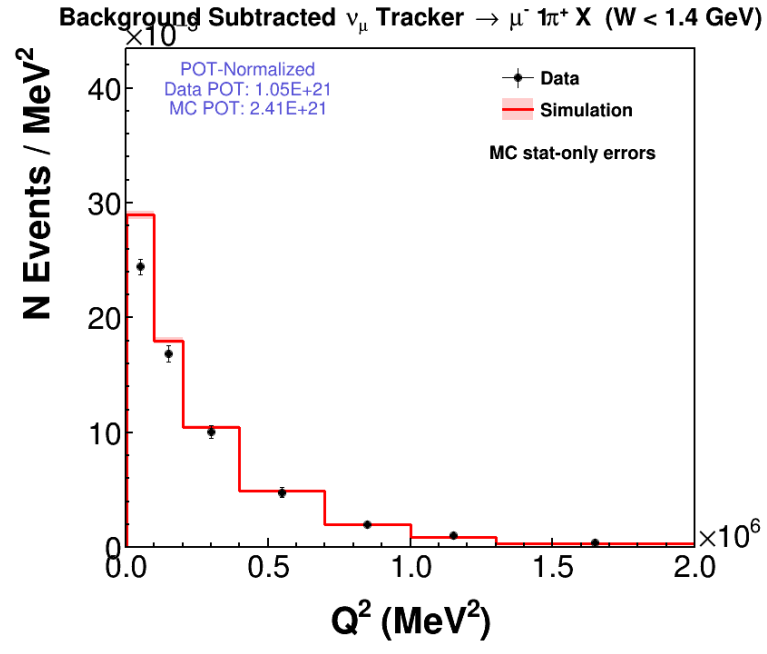

(b) $Q^{2}$ background subtracted event selection 


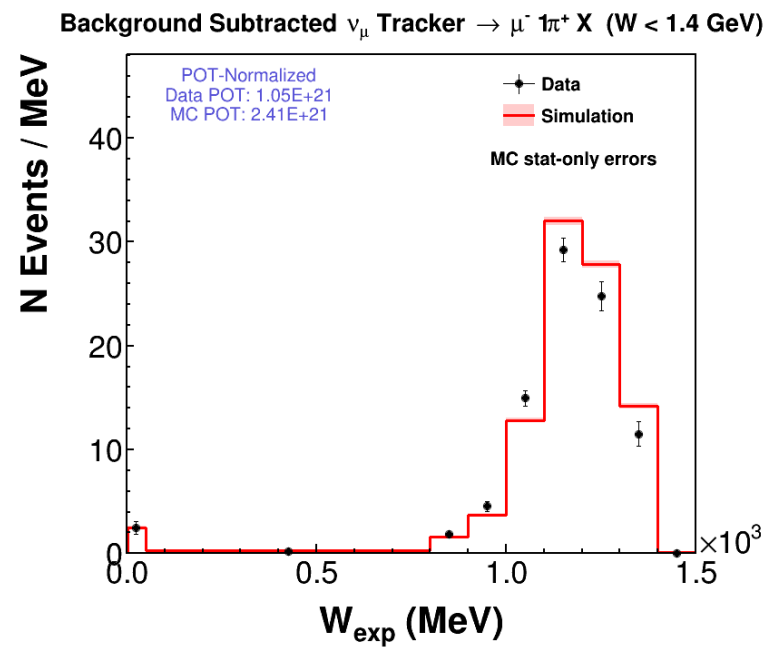

(c) $W_{\exp }$ Background Subtracted

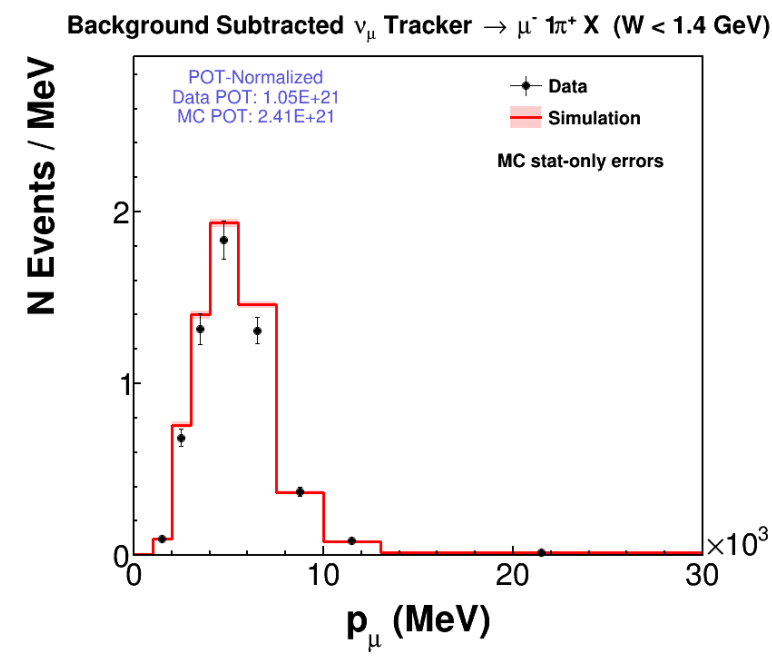

(d) $P_{\mu}$ background subtracted event selection 


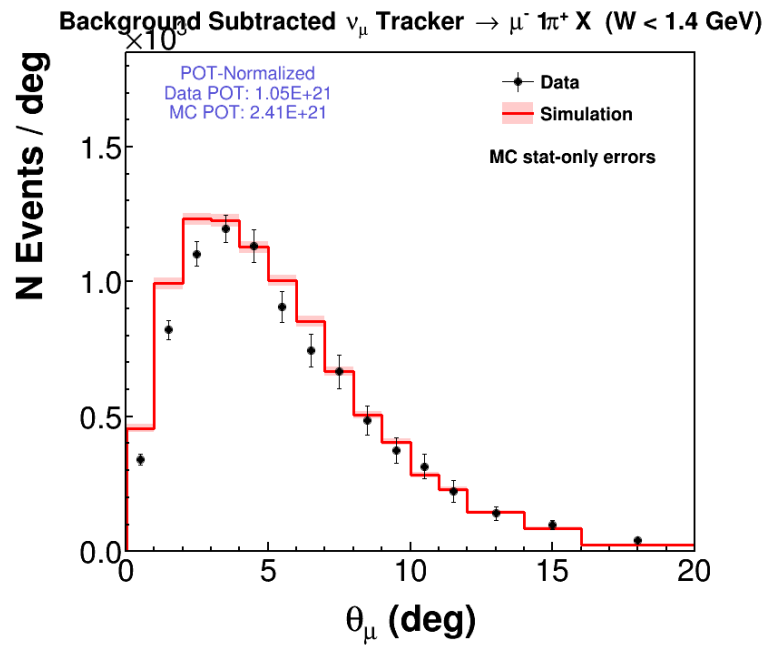

(e) $\theta_{\mu}$ background subtracted event selection

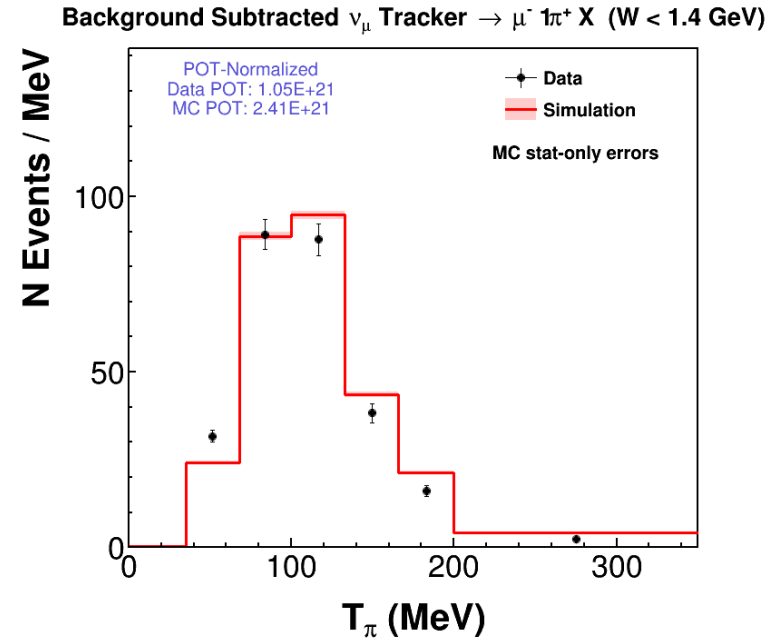

(f) $T_{\pi}$ background subtracted event selection 


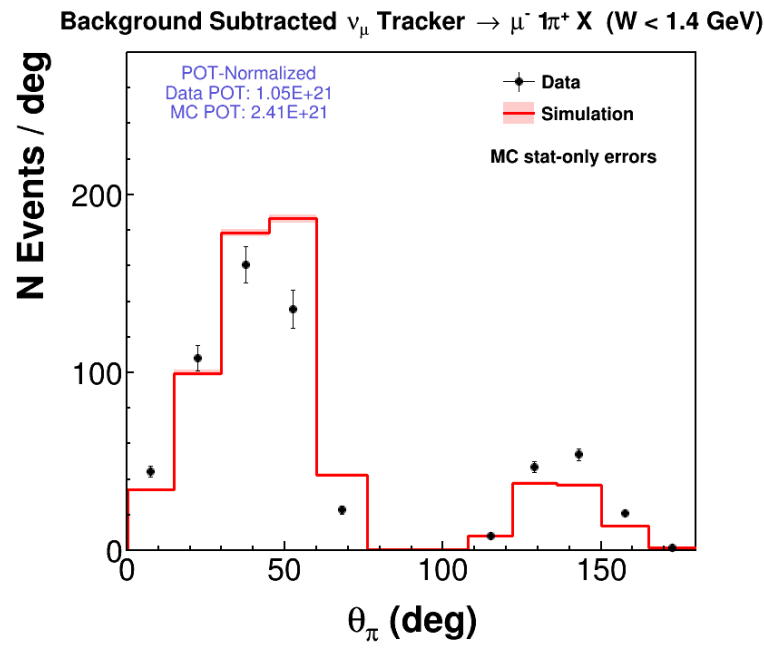

(g) $\theta_{\pi}$ background subtracted event selection

Figure 6.13: Background-subtracted distribution as a function of event variables. 


\subsection{UNFOLDING}

In the process of measuring an event observable, the observable's reconstructed value can differ from its true value. This process is called smearing, and it can cause events to migrate across bins. Various processes in detection, reconstruction, and measurement can cause smearing. Limited detector resolution, for example in the size and geometry of the detector strips, can smear vertex reconstruction or track length determination. The PMT gain measurement is an inherently stochastic process and widens hit energy reconstruction.

The effects of smearing are modeled as well as possible in the detector simulation, and in order to communicate results, for comparisons to theory or to the measurements of other experiments, either detector smearing effects must be undone (in a process called unfolding), or MINERvA's smearing simulation must be provided (to enable forward folding). It is MINERvA's policy to unfold, and thus final results can be compared directly to theoretical predictions.

In this stage, first a smearing or migration matrix is constructed from Monte Carlo events whose reconstructed properties pass event selection cuts and whose truth properties satisfy the signal definition. The events of this Monte Carlo sample fill a 2D migration histogram in bins of [reconstructed bin, truth bin]. 


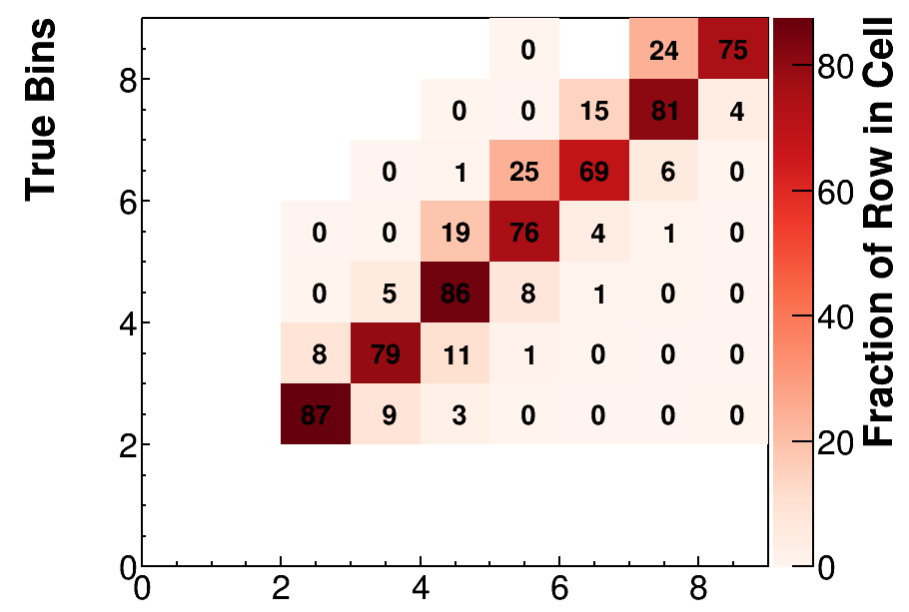

Reco Bins

(a) $E_{\nu}$ Migration Matrix.

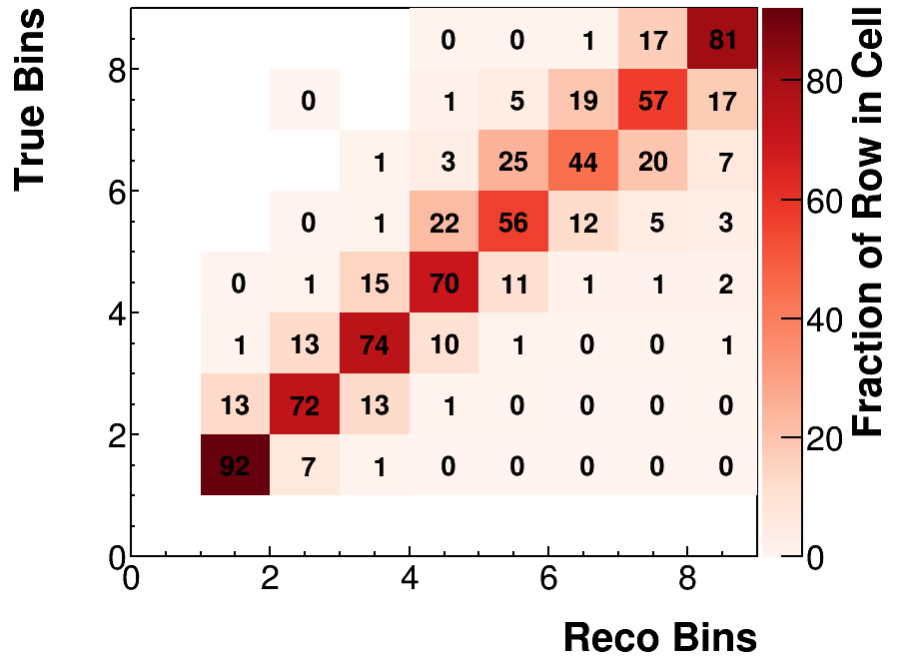

(b) $Q^{2}$ Migration Matrix. 


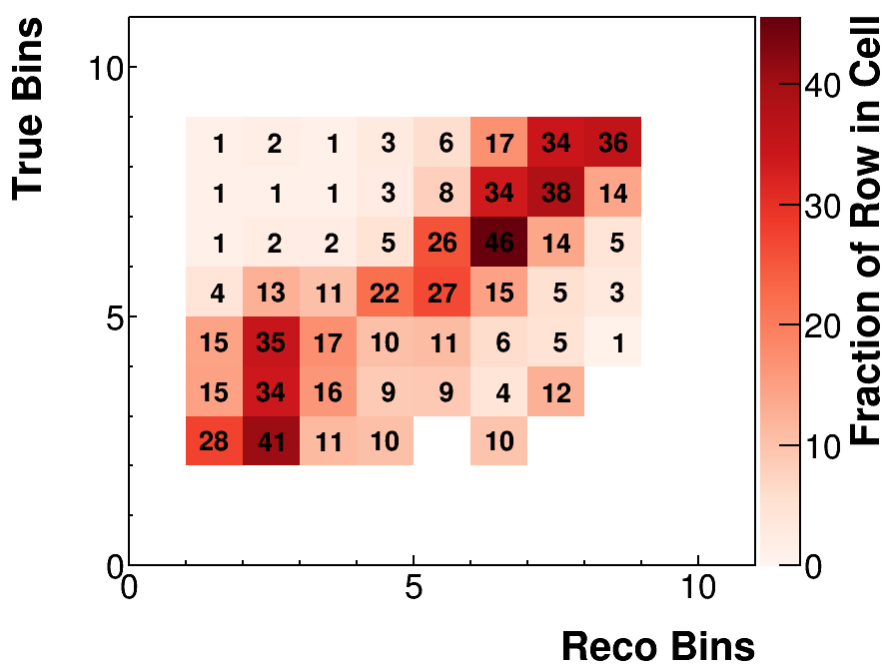

(c) $W_{\exp }$ Migration Matrix

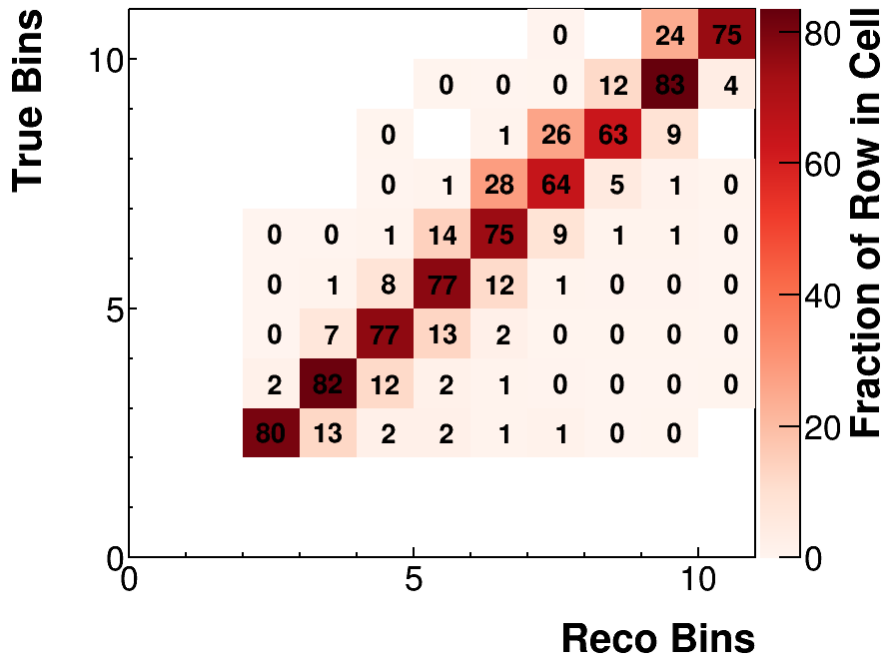

(d) $P_{\mu}$ Migration Matrix. 


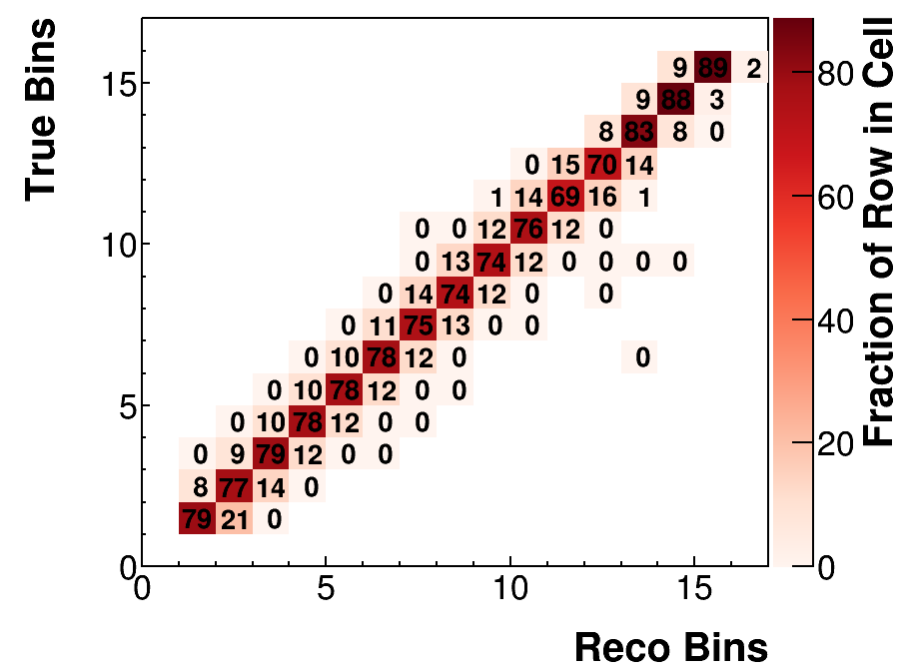

(e) $\theta_{\mu}$ Migration Matrix.

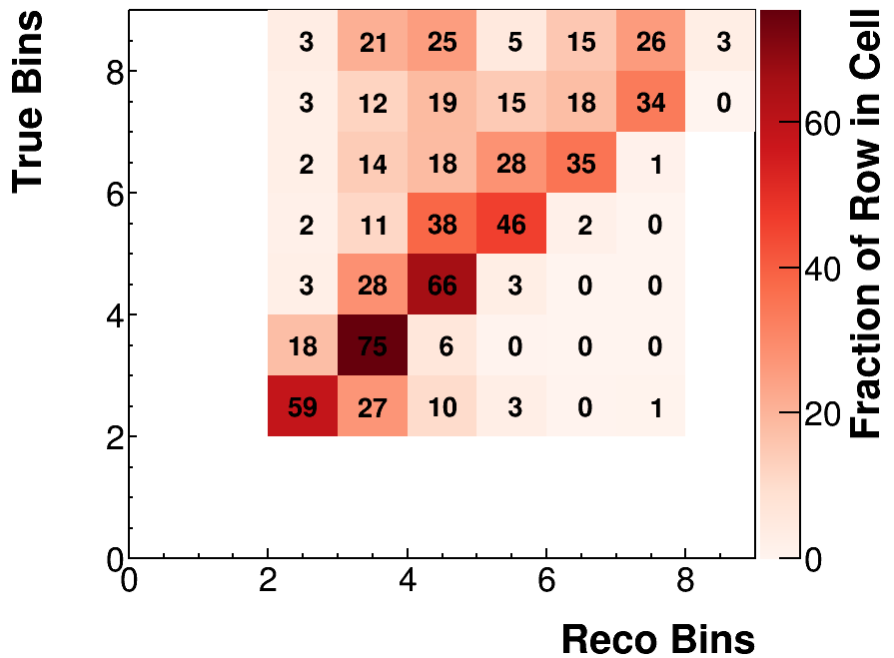

(f) $T_{\pi}$ Migration Matrix. 


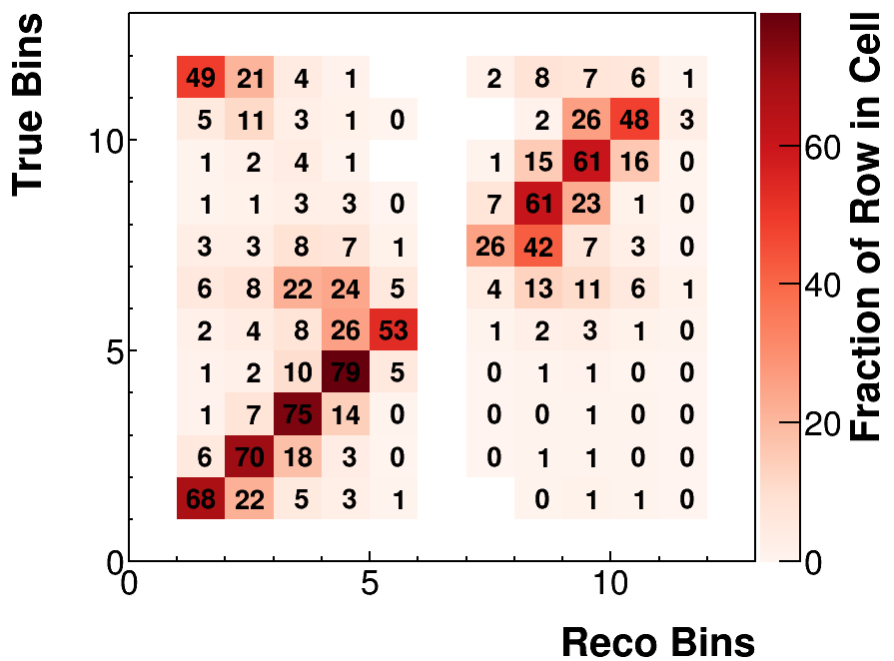

(g) $\theta_{\pi}$ Migration Matrix.

Figure 6.14: Migration matrices for all event variables. 
Represented as a matrix $M$ when left-multiplying on the 1D truth distribution, the migration smears or folds the truth distribution $\hat{\lambda}$ into a reconstructed distribution, $\hat{y}$.

$$
\hat{y}=M \hat{\lambda}
$$

The migration matrix is numerically inverted to obtain the unfolding matrix, $U$, which can then be applied to the background-subtracted reconstructed values to unsmear them into true bins:

$$
\hat{\lambda}=U \hat{y}
$$

Written in the notation of the cross section formula Equation 6.1, the unfolding matrix is summed over reconstructed bins $j$, to obtain the signal content of true bin $\alpha$ :

$$
N_{\alpha}^{\text {true, signal }}=\sum_{j} U_{j \alpha}\left(N_{\text {data }, j}-N_{\text {sim }, j}^{\text {bkgd }}\right)
$$

The method used to invert the migration matrix is iterative expectation-maximization. Regularization, the reduction of variance at the cost of increasing bias, is accounted for by early stopping. The method is implemented by the RooUnfold ROOT package. This analysis uses four iterations that were chosen by finding the iteration $k$ after which the bias stabilized, where the bias is given by:

$$
\chi^{2}=\left(x^{k}-x^{\text {true }}\right)_{j} V_{j \alpha}^{-1}\left(x^{k}-x^{\text {true }}\right)_{\alpha}
$$

and where $V$ is the unfolding covariance matrix.

To validate the unfolding procedure, the migration matrix was used to unfold warped Monte Carlo distributions, the biases were checked for stability, and the warped simulation was recovered within four iterations. Four warped samples were formed by toggling or doubling the following event weights (see Sections 5.4 and Chapter 7): the non-resonant pion production weight (amplify), the resonant axial mass $M_{A}^{\text {res }}$ genie tolerance (amplify), the low $Q^{2}$ pion production weight (toggle), and the anisotropic resonance decay genie tolerance (toggle). 
The unfolding procedure is sensitive to statistical uncertainties in the Monte Carlo sample, and the uncerainties tend to inflate upon each iteration. This in turn causes fluctuations in the unfolded bins, which are then offset in neighboring bins during normalization. All of this causes correlations in the statistical uncertainties between bins in the unfolded spectrum. The correlations are captured in a covariance matrix which is propagated through the rest of the cross section calculation. Figure 6.15 shows the unfolded distributions. 


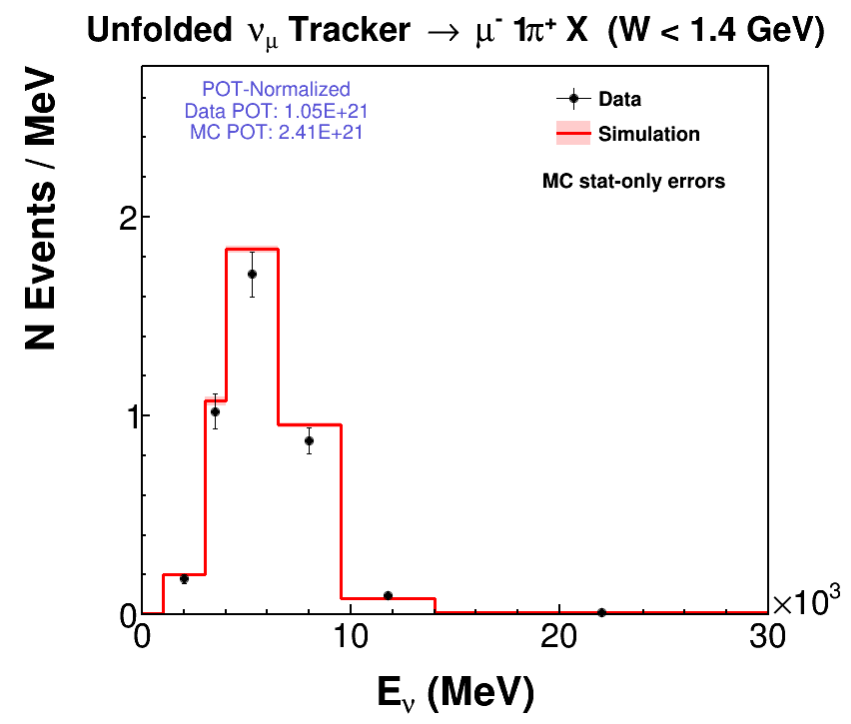

(a) $E_{\nu}$ unfolded data

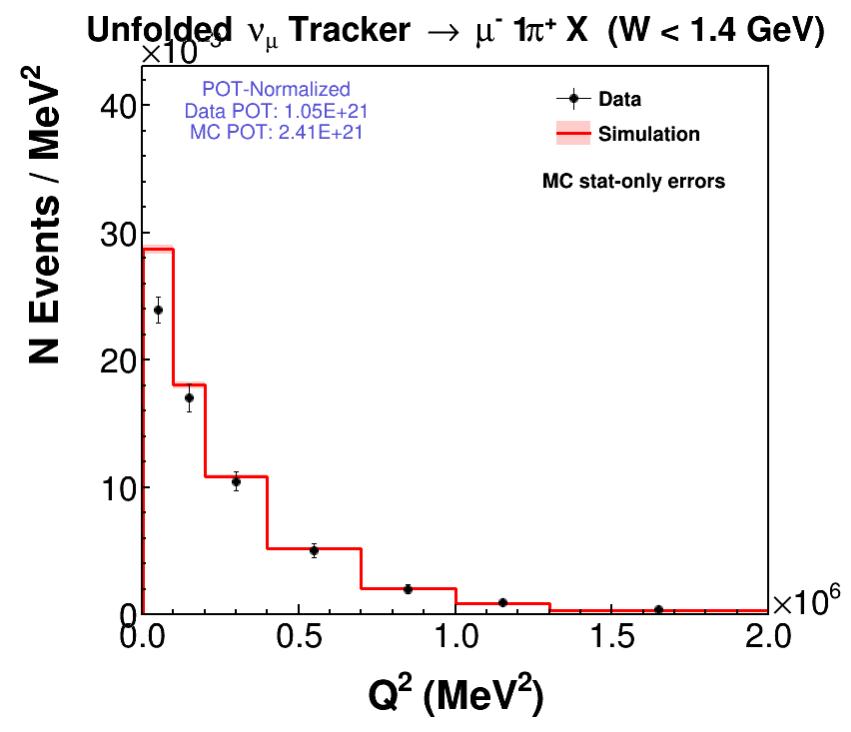

(b) $Q^{2}$ unfolded data 


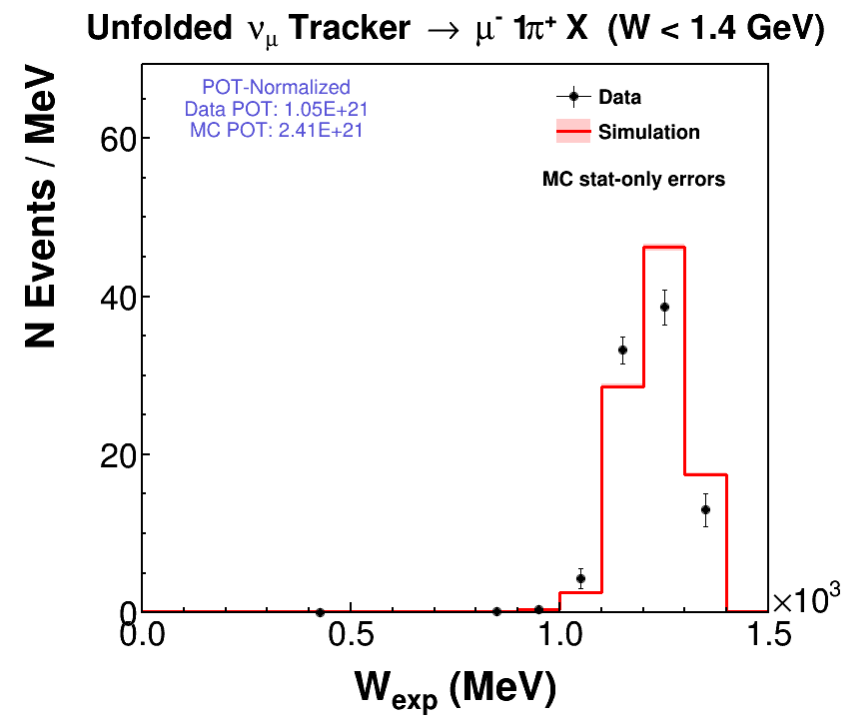

(c) $W_{\text {exp }}$ unfolded data

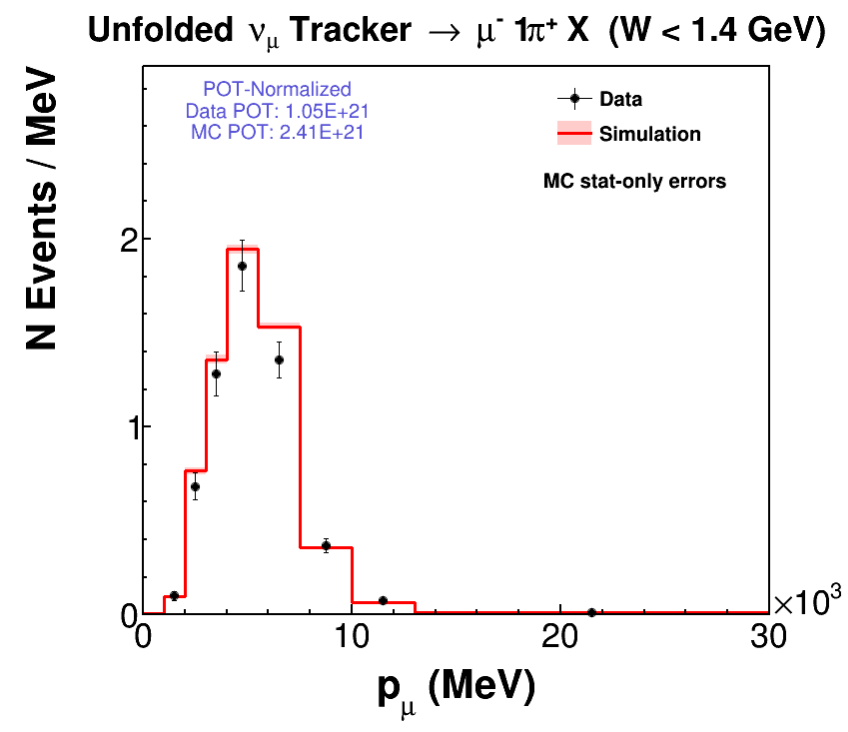

(d) $P_{\mu}$ unfolded data 


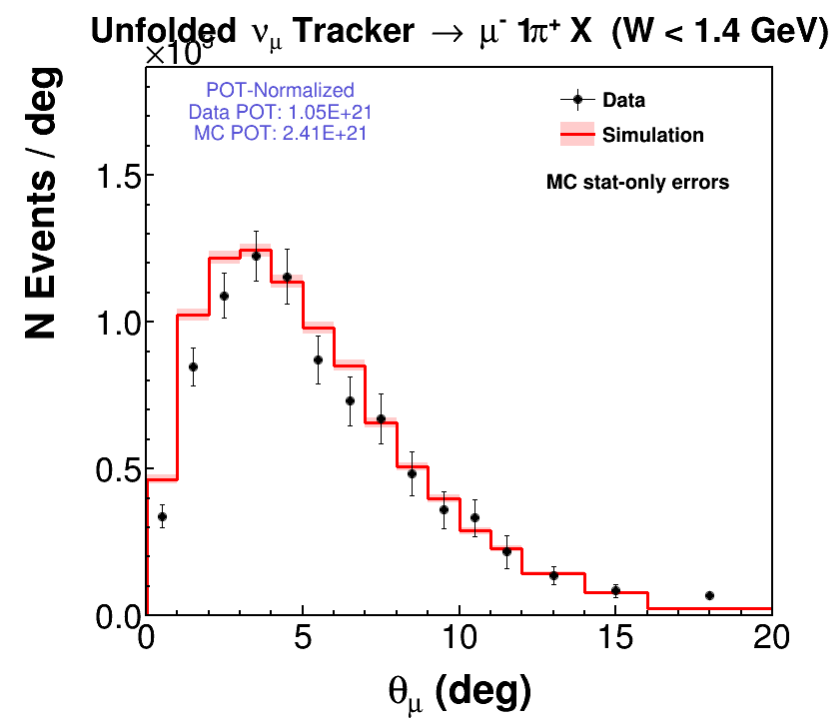

(e) $\theta_{\mu}$ unfolded data

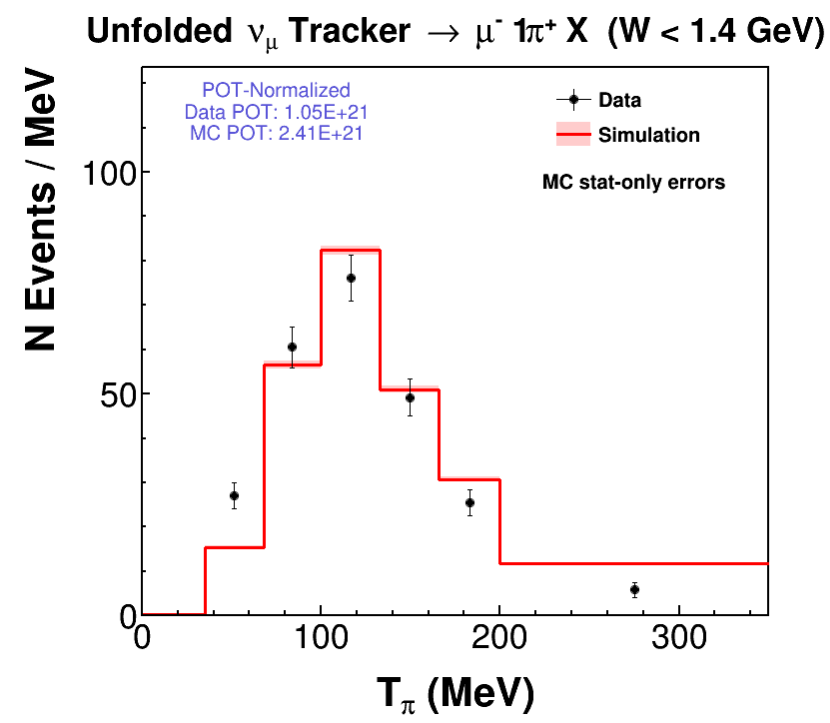

(f) $T_{\pi}$ unfolded data 


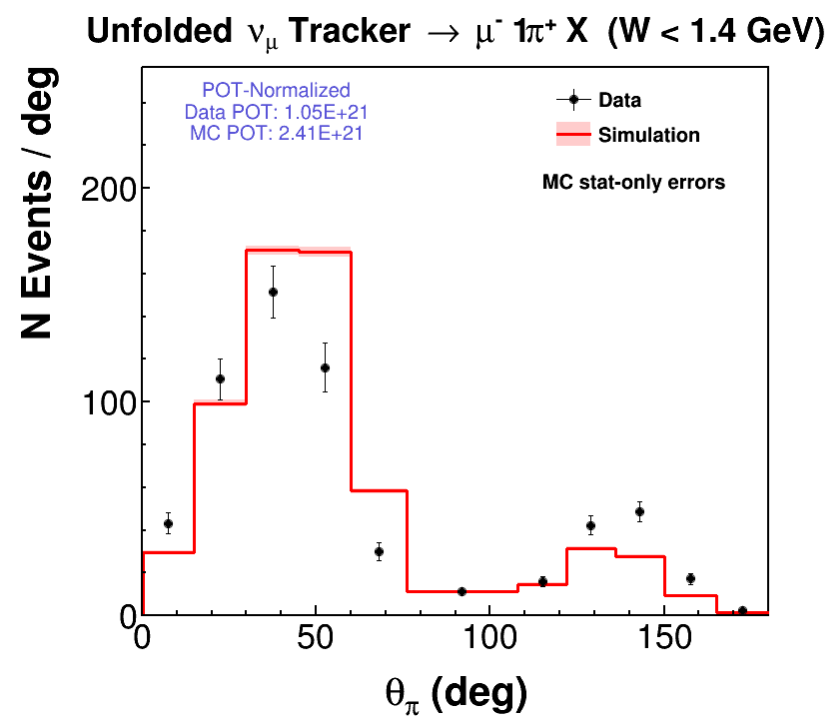

(g) $\theta_{\pi}$ unfolded data

Figure 6.15: Unfolded distributions for all analysis variables. 


\subsection{EFFICIENCY CORRECTION AND NORMALIZATION}

The purpose of the efficiency correction stage is to repopulate the unfolded data with all the signal events that were removed by selection cuts or undetected due to detection thresholds or geometric detector acceptances. The missing signal events are predicted from the Monte Carlo Simulation. As discussed in 6.2, the acceptance correction will mainly fill in missed signal events outside of the fiducial volume cut, below the muon momentum detection threshold, and at perpendicular pion angles. The efficiency correction is formed as

$$
\epsilon=\frac{N^{\text {Selected Signal }}}{N^{\text {Total Signal }}}
$$

and is a function of event variables. Efficiency distributions are shown in Figure 6.16.

In the final stage of the cross section calculation the efficiency-corrected distributions are divided by the integrated flux prediction scaled to the dataset's POT exposure and by the total number of target nucleons. The flux normalization includes, of course, error bars and benefits from the immprovements to the flux simulation described in Appendix A. The number of nucleons is estimated using from the measured densisty of the detector materials. The values of the normalization constants are given in Table 6.3.

In the case of the cross section with respect to $E_{\nu}$, the number of events in each bin is normalized by the flux in the corresponding bin.

The final cross section results are presented in Chapter 8 following a discussion of the measurement error analysis in Chapter 7. 


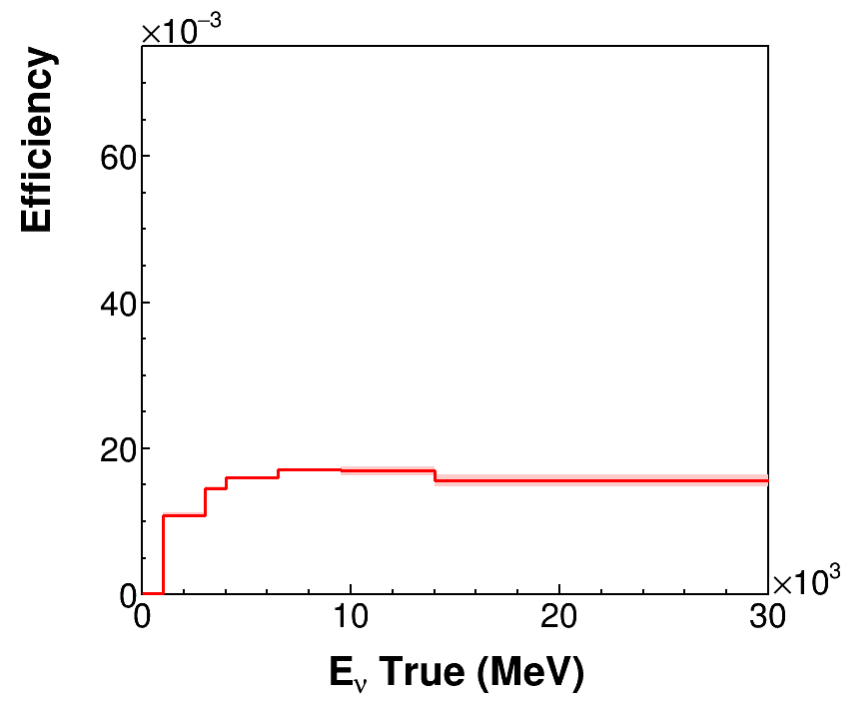

(a) $E_{\nu}$ efficiency correction

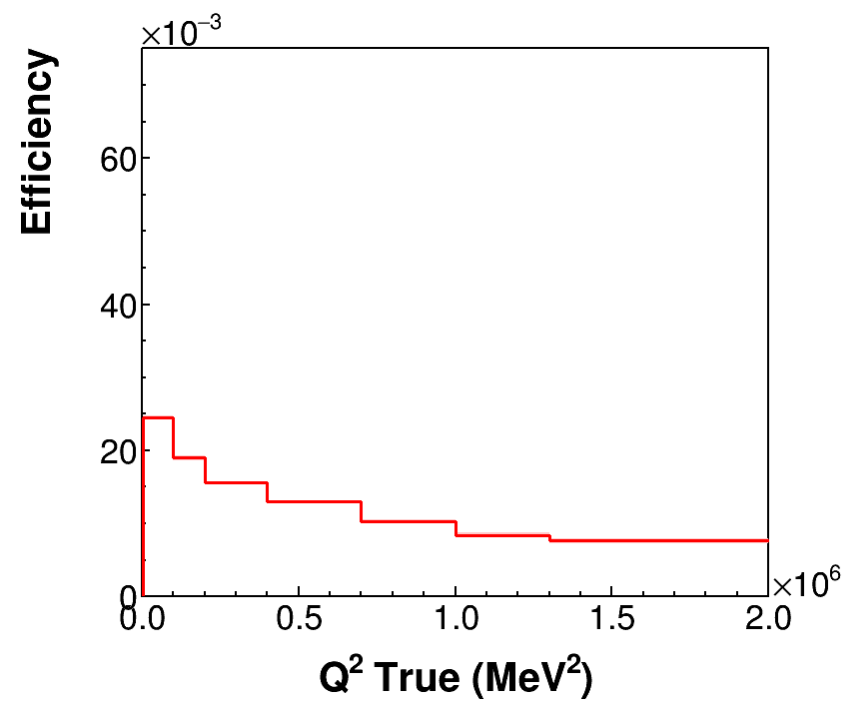

(b) $Q^{2}$ efficiency correction 


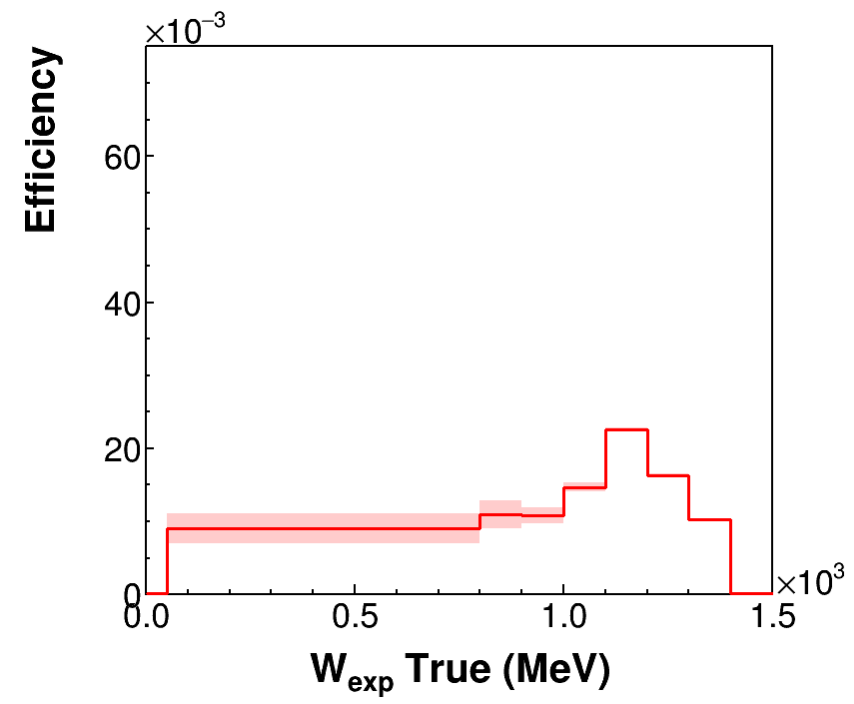

(c) $W_{\text {exp }}$ efficiency correction

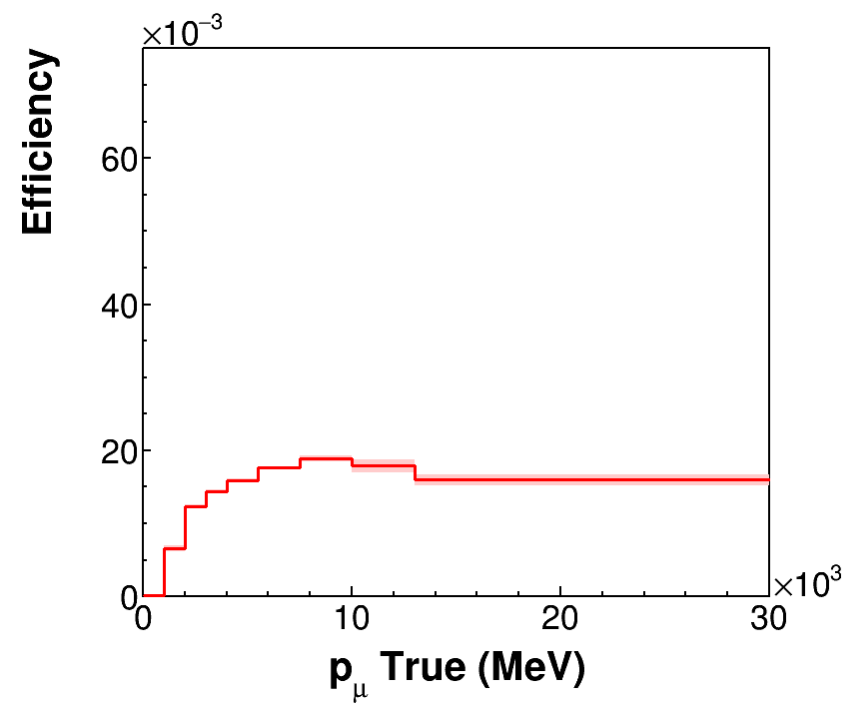

(d) $P_{\mu}$ efficiency correction 


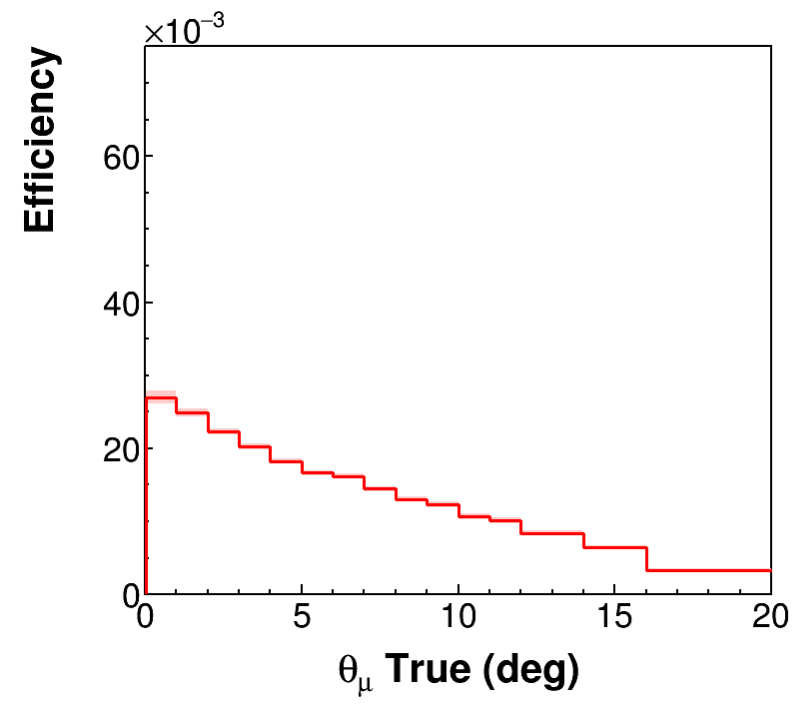

(e) $\theta_{\mu}$ efficiency correction

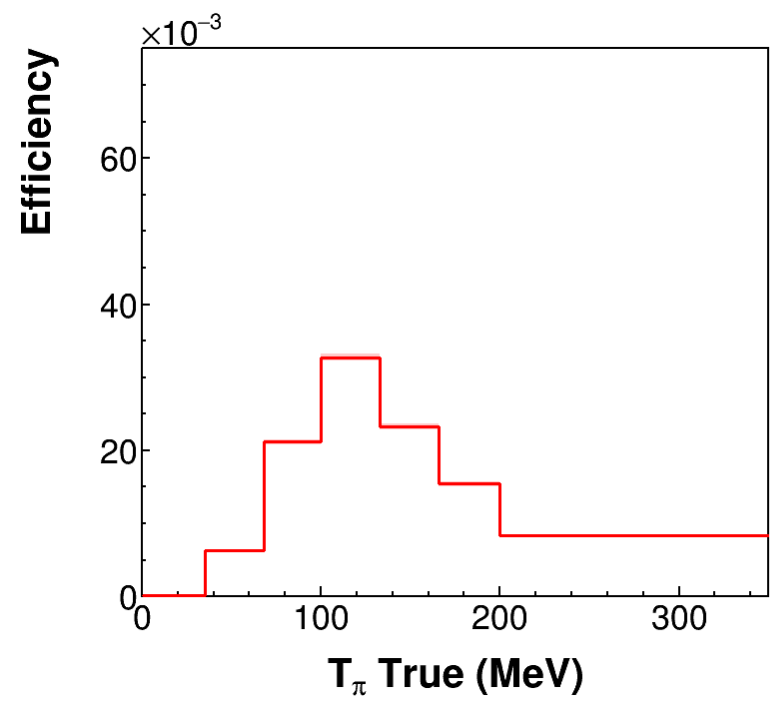

(f) $T_{\pi}$ efficiency correction 


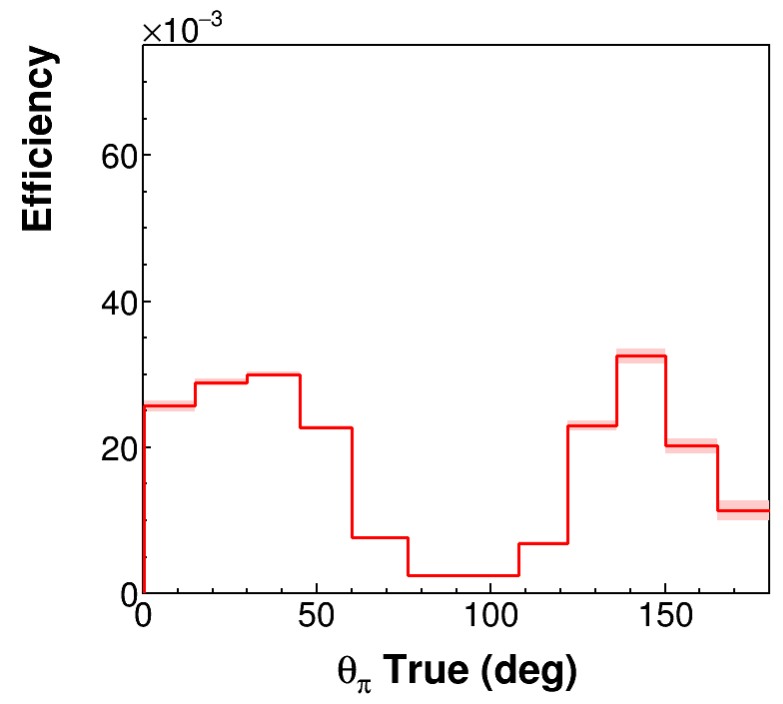

(g) $\theta_{\pi}$ efficiency correction

Figure 6.16: Efficiencies as a function of event variables.

Table 6.3: Cross section normalization constants

\begin{tabular}{l|c} 
Protons on target (data) & $1.05 \mathrm{e}+21$ \\
Protons on target (MC) & $2.33 \mathrm{e}+21$ \\
Number of nucleons & $3.47403 \mathrm{e}+30$ \\
Integrated Flux & $6.91356 \mathrm{e}-08 \nu_{\mu} / \mathrm{cm}^{2} /$ POT
\end{tabular}




\subsection{ERROR ANALYSIS}

In this chapter, the method of calculating cross section errors, called the many-universe method is introduced (7.1). Then the three major categories of uncertainties: model (7.2), detector (7.3), and flux (7.4) uncertainties are presented in detail. Cross section uncertainties are presented at the end, in Section 7.5

\subsection{MANY-UNIVERSE UNCERTAINTY METHOD}

MINERvA employs a many-universe method of error propagation. In this method the complete cross section pipeline is re-calculated many times - each time in a different universe in which a source of systematic error is shifted in the simulation by some number of standard deviations away from its measured, i.e. central value.

The creation of shifted-universe samples is performed either by the adjustment of event weights or by the smearing of event observables. In the former case, for example, the overall rate of neutral current resonance production is shifted by $\pm 20 \%$, so any neutral current resonance events (which are background events) will be weighted by 0.8 in the "down" universe and by 1.2 in the "up" universe. In the latter case, for example, the uncertainty in the detector mass affects the muon energy (among other things), shifting it $\pm 11 \mathrm{MeV}$. Such shifted kinematics mean that an event will shift bins or it may fail a cut that the central value passed.

Two universes are considered for each source of systematic uncertainty, corresponding to an up-shift and a down-shift relative to the central value. The uncertainty on the flux measurement is an exception, using 100 universes, as will be discussed below. 
After each step of the cross section calculation, including the final cross section, the total systematic uncertainty can be evaluated as the sum in quadrature of the uncertainty from each source. The uncertainty for each source is taken from a $N \times N$ covariance matrix, where $N$ is the number of variable bins. The covariance matrix contains the uncertainties in each bin along the diagonal and the correlations between bins in the off-diagonals. The $i, j$ element of the covariance matrix is given by:

$$
\left.\left.C_{i j}=C_{j i}=\sum_{m}^{M}\left(\left(x_{i, m}-\bar{x}_{i}\right)\right)-\left(x_{j, m}-\bar{x}_{j}\right)\right)\right)
$$

where $M$ is the number of universes, $x_{i, m}$ is the bin content of bin $i$ and universe $m . x_{i, m}$ is compared to $\bar{x}_{i}$, the mean of the $i$ th bin across all universes. The uncertainty in the $k$ th bin is the square root of the $k, k$ element. 


\subsection{MODEL UNCERTAINTIES}

In the generation of the $\mathrm{MC}$ dataset, the GENIE event generater supplies many event weights corresponding to the uncertainties on physics input parameters. The weights are created by propagating a parameter "knob turn" through the event generation. In total, 31 GENIE knobs turns are considered - two universes for each knob.

The knobs control two categories of effects: interaction model and final state interactions. The interaction model knobs control aspects of the initial neutrino-nucleon interaction probability distribution, for example the rates of elastic scattering and quasi-elastic scattering. The final state interaction model knobs adjust post-neutrino-interaction effects, such as mean free paths or rates of charge exchange or absorption.

Interaction model uncertainties on the cross section measurement are 3-6\%, and FSI uncertainties are lower, typically $1-4 \%$.

\subsubsection{Interaction model}

Interaction model uncertainties are due mostly to contributions from $\nu+p$ non-resonant two pion production and from the axial and vector form factors on resonance and charged current quasielastic production.

Non-resonant pion production is modeled in GENIE with the scaled Bodek-Yang model [36]. For non-resonant single- $\pi$ production, the model is adjusted to reproduce neutrino-deuterium bubble chamber measurements as explained in Sections 5.4.3 and 4.4.2. However, nonresonant two- $\pi$ production is not so constrained and the $\pm 50 \%$ weight on the channel rate contributes to a $\sim 2-3 \%$ uncertainty at high- $W_{\exp }$ where more energy is available for particle production.

GENIE uses the Rein-Sehgal model of neutrino induced nucleon resonances [104], which is parameterized with separate axial and vector form factors, each with free mass parameters, $M_{A}^{\text {res }}$ and $M_{V}^{\text {res }}$ as in 2.25 .

The default masses are $M_{A}^{\text {res }}=1.12 \mathrm{GeV}$ and $M_{V}^{\text {res }}=0.84 \mathrm{GeV}$. Tolerances on these masses are $20 \%$ on $M_{A}^{\text {res }}$ and $10 \%$ on $M_{V}^{\text {res }}$, though fits to electron-nucleus scattering suggest 
the latter uncertainty is too large [54]. The uncertainties due to $M_{A}^{\text {res }}\left(M_{V}^{\text {res }}\right)$ peak at $\sim 5 \%$ $(\sim 3 \%)$.

GENIE uses the Llewellyn-Smith model of charged current quasielastic interactions[80], which is parameterized by an axial form factor as in 2.17

The universe weights on $M_{A}^{C C Q E}$ are $\pm 15 \%$ which lead to a $1-2 \%$ uncertainty on the cross section, primarily from the background model.

The non-resonant two-pion production and resonance and charged current quasielastic form factor masses dominate the interaction model uncertainties. Table 7.1 lists the full set of GENIE interaction model uncertainties considered.

Table 7.1: GENIE interaction model uncertainty sources

\begin{tabular}{|c|c|c|}
\hline Source & Variation & Cross Section Effect \\
\hline \multicolumn{3}{|c|}{ CC Quasielastic } \\
\hline Axial mass & $\begin{array}{r}-15 \% \\
+25 \%\end{array}$ & $1-5 \%$ \\
\hline Vector form factor model & $\mathrm{BBBA}^{+25 \%} \rightarrow$ Dipole & $<0.5 \%$ \\
\hline Pauli suppression & $\pm 30 \%$ & $<0.5 \%$ \\
\hline \multicolumn{3}{|c|}{ NC Elastic } \\
\hline Axial mass & $\pm 25 \%$ & $<0.5 \%$ \\
\hline Strange axial form factor $\eta$ & $\pm 30 \%$ & $<0.5 \%$ \\
\hline \multicolumn{3}{|c|}{ Resonance } \\
\hline CC Normalization & $\pm 20 \%$ & $0-2 \%$ \\
\hline NC Normalization & $\pm 20 \%$ & $<0.5 \%$ \\
\hline Axial mass & $\pm 20 \%$ & $1-5 \%$ \\
\hline Vector mass & $\pm 20 \%$ & $1-3 \%$ \\
\hline \multicolumn{3}{|c|}{ CC \& NC Non-Resonant Pion Production } \\
\hline Normalization of $1-\pi$ final states & $\pm 5 \%$ & $<0.5 \%$ \\
\hline Normalization of $2-\pi$ final states $(\nu+p)$ & $\pm 50 \%$ & $2-3 \%$ \\
\hline Normalization of $2-\pi$ final states $(\nu+n)$ & $\pm 50 \%$ & $<0.5 \%$ \\
\hline
\end{tabular}

\subsubsection{Final state interactions}

Final state interaction uncertainties are due primarily to uncertainty in the angular dependence of the $\Delta$ decay ( $\sim 2 \%$ effect) and uncertainties in final state pion fates from absorption, 
inelastic scattering, and mean free path (total $\sim 1.5 \%$ effect).

In GENIE by default the $\Delta \rightarrow \pi N$ decay is anisotropic with the pion angular distribution $W_{\pi}(\cos (\theta))$ expressed as:

$$
W_{\pi}(\cos (\theta))=1-p\left(\frac{3}{2}\right) P 2(\cos (\theta))+p\left(\frac{1}{2}\right) P 2(\cos (\theta))
$$

where $\theta$ is the pion production angle in the $\Delta$ center of mass frame, $P 2$ is the Legendre polynomial, and $p\left(\frac{3}{2}\right)$ and $p\left(\frac{1}{2}\right)$ are coefficients for each state of $\Delta$ angular momentum. In the default case, $p\left(\frac{3}{2}\right)=0.75$ and $p\left(\frac{1}{2}\right)=0.25[27]$. Due to a bug in the Monte Carlo weight calculation, the down-knob and up-knob universes don't have physical interpretation, but they likely overestimate the uncertainty in the anisotropy. While the contribution of this uncertainty is largest among the final state interaction uncertainties, it's small compared to the total statistical $\oplus$ systematic uncertainties. This should be fixed in future work.

The other non-trivial final state interaction uncertainties are due to the rates of pion inelastic scattering and absorption within the nuclear medium. The signal definition is defined by the number of final state pions, so events with initial pions that change identity or are absorbed within the nucleus are not signal events. The mean free path of pions within the nucleus governs the likelihood of final state interactions occurring before the pion escapes. Similar uncertainties on nucleons within the nucleus have a small effect on the cross section.

Table 7.2 lists the full set of GENIE FSI uncertainties considered. 
Table 7.2: GENIE FSI uncertainty sources

\begin{tabular}{|c|c|c|}
\hline Source & Variation & Cross Section Effect \\
\hline \multicolumn{3}{|c|}{ Nucleon Fates } \\
\hline Mean free path & $\pm 20 \%$ & $<0.5 \%$ \\
\hline Charge exchange probability & $\pm 50 \%$ & $<0.5 \%$ \\
\hline Elastic interaction probability & $\pm 30 \%$ & $0.5 \%$ \\
\hline Inelastic interaction probability & $\pm 40 \%$ & $<0.5 \%$ \\
\hline Absorption probability & $\pm 20 \%$ & $<0.5 \%$ \\
\hline$\pi$-production probability & $\pm 20 \%$ & $<0.5 \%$ \\
\hline \multicolumn{3}{|c|}{ Pion Fates } \\
\hline Mean free path & $\pm 20 \%$ & $1 \%$ \\
\hline Charge exchange probability & $\pm 50 \%$ & $<0.5 \%$ \\
\hline Elastic interaction probability & $\pm 10 \%$ & $<0.5 \%$ \\
\hline Inelastic interaction probability & $\pm 40 \%$ & $1 \%$ \\
\hline Absorption probability & $\pm 30 \%$ & $1 \%$ \\
\hline$\pi$-production probability & $\pm 20 \%$ & $<0.5 \%$ \\
\hline \multicolumn{3}{|c|}{ Hadronization and Resonance Decay } \\
\hline Resonance $\rightarrow \mathrm{X}+1 \gamma$ branching ratio & $\pm 50 \%$ & $<0.5 \%$ \\
\hline $\begin{array}{l}\text { Pion angular distribution in } \Delta \rightarrow \mathrm{N} \pi \\
x_{F} \text { dependence for } \mathrm{N} \pi \text { final states in }\end{array}$ & see Section 7.2.2 & $2 \%$ \\
\hline AGKY hadronization model & $\pm 20 \%$ & $<0.5 \%$ \\
\hline
\end{tabular}




\subsection{DETECTOR UNCERTAINTIES}

Detector uncertainties are a significant component of total uncertainty, ranging from 410\%. This group is completely dominated by the uncertainty on the measurement of the hadronic recoil energy $E_{\text {recoil }}$ that enters the analysis through the invariant hadronic mass $W_{\text {exp }}$ kinematic cut.

In the Monte Carlo sample, universe shifts in this quantity are calculated by breaking $E_{\text {recoil }}$ into its contributions from each truth source particle and then summing in quadrature the uncertainties from each, weighted by their fractional contribution to the total energy.

Through a test beam experiment[58], an energy scale uncertainty on individual particles was measured. Particle energy scale uncertainties are due to many effects, for example Birks' parameter or detector energy scale calibration. The full list of energy scale uncertainties considered is listed in Table 7.3.

Table 7.3: Particle energy fractional uncertainties

\begin{tabular}{lc} 
Source & Fractional Energy Uncertainty \\
\hline Proton $(\mathrm{KE}<50 \mathrm{MeV})$ & $4 \%$ \\
Proton $(50 \mathrm{MeV}<\mathrm{KE}<100 \mathrm{MeV})$ & $3.5 \%$ \\
Proton $(\mathrm{KE}>100 \mathrm{MeV})$ & $3 \%$ \\
Neutron $(\mathrm{KE}<50 \mathrm{MeV})$ & $20 \%$ \\
Neutron $(50 \mathrm{MeV}<\mathrm{KE}<150 \mathrm{MeV})$ & $10 \%$ \\
Neutron $(\mathrm{KE}>150 \mathrm{MeV})$ & $20 \%$ \\
Pion $(\mathrm{KE}<400 \mathrm{MeV})$ & $5 \%$ \\
Pion $(400 \mathrm{MeV}<\mathrm{KE}<1900 \mathrm{MeV})$ & $4 \%$ \\
Kaon & $4 \%$ \\
Electromagnetic & $3 \%$ \\
Cross Talk & $20 \%$ \\
Other & $10 \%$
\end{tabular}


From the fractional uncertainties on energy sources, the universe shifts are calculated:

$$
\pm \Delta E_{\text {recoil }}=\frac{E_{\text {recoil }}}{\sum_{\text {sources }} E_{s}} \sqrt{\sum_{\text {sources }}\left(\sigma_{s} E_{s}\right)^{2}}
$$

Where the summations are performed over all sources of energy $s$ to $E_{\text {recoil }}$, which include protons, neutrons, pions, kaons, electromagnetic energy, cross talk, and a conservativelyestimated catch-all "other" category. $E_{s}$ is the energy from source $s$, measured by summing the calibrated energy of all non-muon hits in the event. $\sigma_{s}$ is the fractional energy scale uncertainty on source $s$. With $\frac{E_{\text {recoil }}}{\sum_{\text {sources }} E_{s}} \sim 1$ and source uncertainties typically $\sim 5 \%, \Delta E_{\text {recoil }}$ is $\sim 5 \%$ of $E_{\text {recoil }}$.

Muon track angle resolution $\left(0.34^{\circ}\right)$ also contributes to the invariant hadronic mass cut via $\theta_{\mu}$ (see Equation 6.4). In total it contributes a $\sim 0.5 \%$ effect. Other detector uncertainties including detector mass, incident neutrino beam angle, energy loss model, and Birks' parameter together contribute a $<0.5 \%$ effect on the cross section. These uncertainties are further detailed in [45].

The last detector uncertainty of note is from the muon energy determination from MINOS. While MINOS calibration procedures set the uncertainty at $2 \%$, a large data-MC discrepancy in a well-understood, low-recoil process called low- $\nu$ scattering, ultimately led to an adjustment in the muon energy scale and to its uncertainty. The discrepancy, originally thought to be caused by a mismodeling in the flux focusing system, was instead found to be consistent with a shift in the muon energy scale by 1.75 standard deviations. This shift was determined from a joint fit of flux focusing parameters and the muon energy scale, and it further suggested that the uncertainty in the muon energy scale could be decreased from $2 \%$ to $1 \%$. This prescription has been adopted by the MINERvA collaboration, and it has been used for this analysis. The effect of this change is an uncertainty reduction due to the muon energy scale from $\sim 1 \%$ to $\sim 0.5 \%$ on the cross section measurement For more details on the data-MC discrepancy and its resolution, see Section A.2.4. 


\subsection{FLUX}

There are two categories of flux model uncertainties: focusing uncertainties and hadron production uncertainties. Focusing uncertainties refer to imperfect knowledge of the components

of the NuMI beamline (for example location of the focusing horns) or to an otherwise imperfect implementation of the beamline components in the simulation (for example modeling of the focusing horns magnetic fields). The second category, hadron production uncertainties, refers to the uncertainty in the incident proton-target collision that produces the hadrons (in particular pions) that are the ancestors of neutrinos in MINERvA. It includes, too, uncertainties in subsequent reinteractions with the target and surrounding NuMI infrastructure, as well as the kinematics of outgoing hadrons.

Given the many sources of uncertainty in the flux model from both categories, together with the fact that the flux can not be simply re-simulated for each production of the Monte Carlo dataset, it is not feasible to adjust individual sources of flux uncertainty to create downtune and up-tune universes for each, as was done for the model and detector uncertainties. Instead, the flux is simulated 100 times, each time with a gaussian throw of knob turns on its sources of uncertainty. Thus for example, one of the 100 flux universes will be simulated with the focusing horn current shifted up by $1.4 \sigma$, the proton beam spot size will be shifted down $3 \sigma$, and the target y-position will be shifted down $1.2 \sigma$. The difference between each of these universes compared to the central value flux universe determines an event flux weight which is a function of $E_{\nu}$.

At an almost flat 8-10\% across all variables and bins, the flux model constitutes the largest uncertainty in the cross section measurement. Figure 7.1 shows the contributions from focusing and hadron production.

\subsubsection{Hadron production uncertainties}

In the G4NuMI flux simulation package, central value hadron production is simulated with the FTFP-BERT (FRITIOF Precompound - Bertini cascade) inelastic scattering model via G4NuMI's GEANT4. The MINERvA/Fermilab package ppfx[19] makes use of external data 


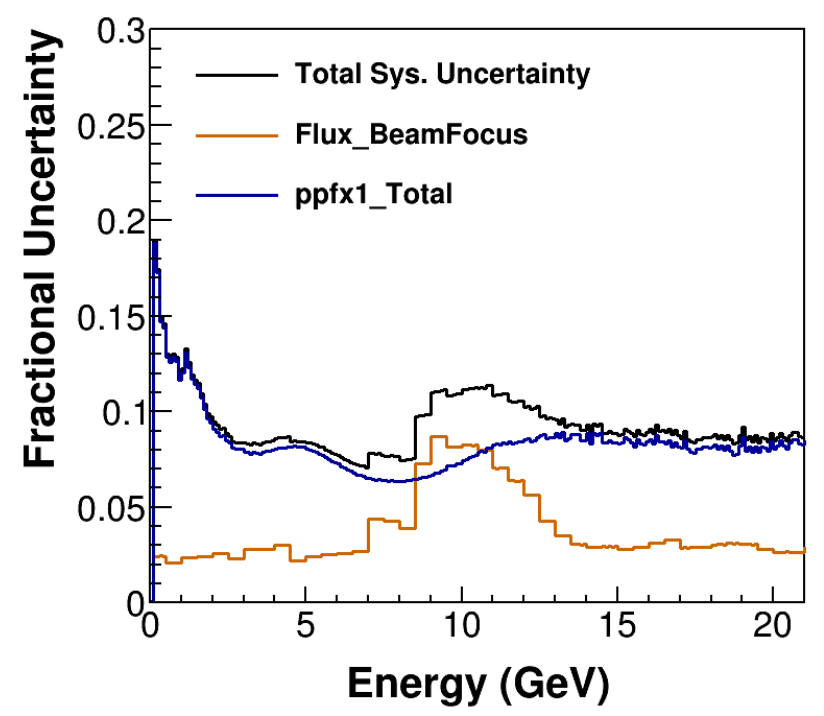

Figure 7.1: Breakdown of the flux model uncertainty into its components: beam focusing and hadron production (ppfx).

to constrain the hadron production simulation.

During the simulation of the flux, this is accomplished by weighting a G4NuMI simulated neutrino to conform its parent hadron to an externally measured hadron yield per incident proton. The weight $w$ is calculated in hadron kinematic bin $k$ (typically Feynman variable $x_{F}$ and transverse momentum $p_{T}$ ):

$$
w(k)=\frac{f_{\text {Data }}(k) s(k)}{f_{\mathrm{MC}}(k)}
$$

where $f$ is the hadron event yield per proton and $s(k)$ is a scale factor from the data and the Monte Carlo proton beam energies. The full set of hadron production effects considered by ppfx include:

- $\boldsymbol{p} \boldsymbol{C} \rightarrow \boldsymbol{\pi} \boldsymbol{X}$ Weights calculated as in 7.4. Uses NA49 data[23] and scaled using alternate hadron production model, FLUKA[48].

- $\boldsymbol{p} \boldsymbol{C} \rightarrow \boldsymbol{K} \boldsymbol{X}$ Weights calculated as in 7.4. Uses NA49 data and scaled with MIPP[100] ratios. 
- $\boldsymbol{p} \boldsymbol{C} \rightarrow$ nucleon $\boldsymbol{X}$ Weights calculated as in 7.4. Uses NA49 data[23] and scaled using alternate hadron production model, FLUKA[48].

- $\boldsymbol{n} \boldsymbol{C} \rightarrow \boldsymbol{\pi} \boldsymbol{X}$ Weights estimated through isoscalar symmetry with $\sigma\left(p C \rightarrow \pi^{ \pm}\right)=$ $\sigma\left(n C \rightarrow \pi^{\mp}\right)$.

- Nucleon- $\boldsymbol{A}$ Weights on the interactions of nucleons with other materials in the NuMI beamline, including $\mathrm{pC}$ interactions where no data is available. Weights estimated by nuclear mass scaling[22].

- Meson-incident Little external data is available. The default FTFP estimation is used with $40 \%$ uncertainty taken from proton-incident data-Monte Carlo differences.

- Target attenuation A weight is applied based on inclusive interaction rates for hadrons. Uses data from several precise datasets which agree with the Monte Carlow to $10 \%$.

- Absorption Similar to target attenuation treatment.

- Other Rare interactions not falling into any above category. A $40 \%$ uncertainty is assumed.

The ppfx-hadron production uncertainty breakdown is shown in Figure 7.2.

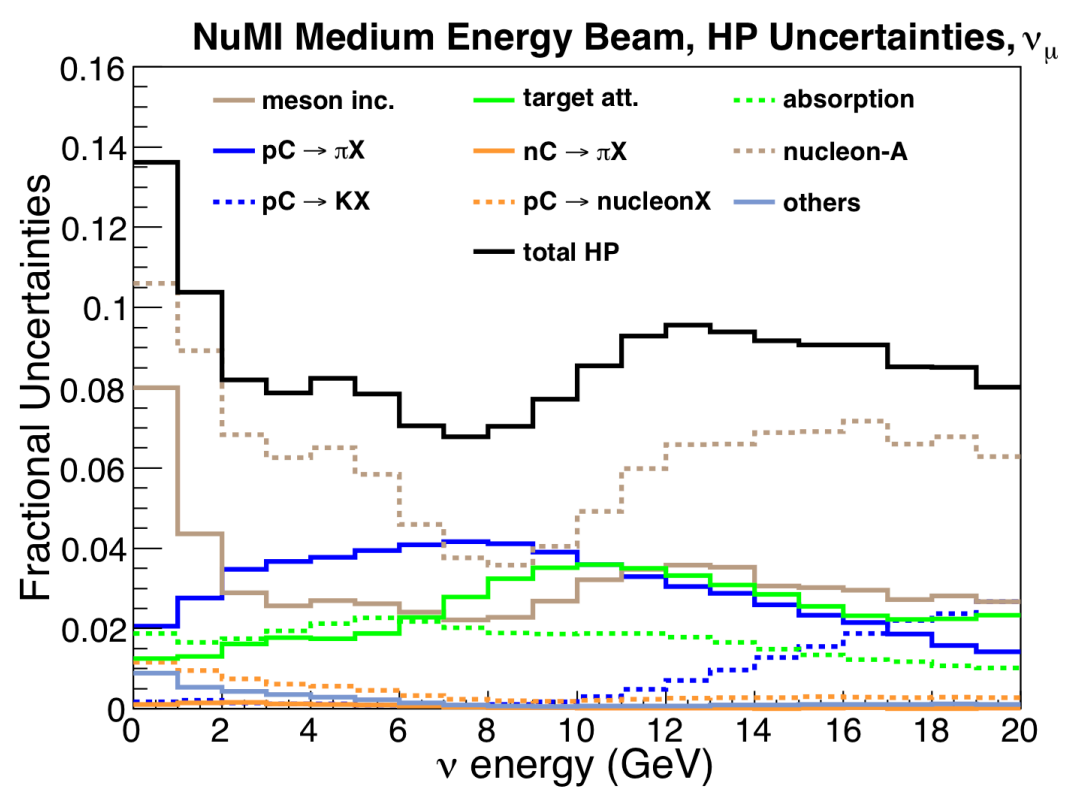

Figure 7.2: Breakdown of the hadron production flux uncertainty. 


\subsubsection{Focusing uncertainties}

G4NuMI implements a sophisticated beamline geometry model through which a neutrino's full particle ancestry is simulated.

The uncertainty due to a focusing component is calculated first by generating a flux with the value of that component shifted from its central value by the stated uncertainty in the knowledge of that parameter. The uncertainty then is taken to be the ratio of the shifted flux to the nominal. ${ }^{1}$

The list of uncertainty sources considered is listed in Table 7.4. A detailed study of the focusing uncertainties is given in Appendix A.

\footnotetext{
${ }^{1}$ Other methods to calculate the uncertainty were also considered. For example fluxes were shifted by $\pm 1 \sigma, \pm 2 \sigma$, and $\pm 3 \sigma$, and a polynomial fit was made to find the flux's dependency on the parameter. As another example, fluxes were shifted by $\pm 1 \sigma$ and the mean of the absolute values of the shifted fluxes were taken to be the uncertainties. Ultimately the $+1 \sigma$ method was adopted due to computing limitations and simplicity when the more complex methods did not significantly affect results.
} 
Table 7.4: Beamline focusing parameters

\begin{tabular}{lcc} 
Parameter & Nominal Value & Shifted Amount \\
\hline Focusing horn current & $200 \mathrm{kA}$ & $1 \mathrm{kA}$ \\
Focusing horn water layer & $1 \mathrm{~mm}$ & $0.5 \mathrm{~mm}$ \\
Horn 1 x position & $0 \mathrm{~mm}$ & $0.5 \mathrm{~mm}$ \\
Horn 1 y position & $0 \mathrm{~mm}$ & $0.5 \mathrm{~mm}$ \\
Horn 1 z position & $0 \mathrm{~mm}$ & $2.0 \mathrm{~mm}$ \\
Horn 2 x position & $0 \mathrm{~mm}$ & $1.0 \mathrm{~mm}$ \\
Horn 2 y position & $0 \mathrm{~mm}$ & $1.0 \mathrm{~mm}$ \\
Proton beam spot size & $1.4 \mathrm{~mm}$ & $0.2 \mathrm{~mm}$ \\
Proton beam x position & $0 \mathrm{~mm}$ & $0.4 \mathrm{~mm}$ \\
Proton beam y position & $0 \mathrm{~mm}$ & $0.4 \mathrm{~mm}$ \\
POT counting & & $2 \%$ \\
Target x position & $0 \mathrm{~mm}$ & $0.5 \mathrm{~mm}$ \\
Target y position & $0 \mathrm{~mm}$ & $0.5 \mathrm{~mm}$
\end{tabular}




\subsection{CROSS SECTION UNCERTAINTIES}

Cross section systematic uncertainties are shown in Figure 7.3. Detector and GENIE model uncertainty breakdowns are shown in Appendix B.

The flux uncertainty is the dominant uncertainty in this analysis. It is mostly flat across all variables at $8-10 \%$. This value is comparable to LE-era measurements. Despite significant hadron production uncertainties to the flux from the ppfx package [19] it is not reduced relative to LE-era uncertainty for two reasons. First, the ME focusing uncertainties are inherently more sensitive to focusing systematics (despite also improved and mostly reduced uncertainties in focusing parameters). Compare Figure A.1 with A.2 or 7.1. Second, during the preparation of this thesis, a method to constrain the flux normalization and uncertainty was being standardized by the MINERvA experiment and has since been published [123]. The procedure uses a measurement of the well-known process of $\nu-e$ elastic scattering to make the constraint. Future work will incorporate this constraint, which is expected to reduce the flux uncertainty to $<5 \%$.

For most variables the second-largest uncertainty is due to detector effects. As discussed in 7.3 this uncertainty is dominated by the particle response uncertainty via difficulties in reconstructing $E_{h a d}$, which enters the analysis via the $W_{\text {exp }}$ cut. The detector uncertainty rises to $25 \%$ in the highest bin of $T_{\pi}$. In this case, the large particle response uncertainty is likely due directly to track energy reconstruction.

GENIE interaction model uncertainties are also a significant contribution. In particular, they are significant across the range of $W_{\exp }$ (Figure B.2c) and at high- $Q^{2}$ (Figure B.2b). For $W_{\text {exp }}$, axial and vector resonant mass uncertainties dominate at low- $W_{\text {exp }}$, while $2 \pi$

production normalization dominates, naturally, at high- $W_{\text {exp }}$. At high- $Q^{2}$, the axial and vector masses of CCQE and Resonance dominate.

As discussed in Section 6.5 bin sizes were chosen to diagonalize the migration matrix while also setting the statistical uncertainty to be comparable to the systematic uncertainty in that bin. The result of this compromise is that statistical uncertainties are somewhat shape-dependent for different variables, ranging from $1 \%$ up to $10 \%$. In $E_{\nu}$ and $P_{\mu}$ statistics are very high around the flux peak, and consequently bin sizes are limited by their resolution. 
It is interesting to observe that statistical uncertainties and all systematic uncertainties are mostly flat across $\theta_{\pi}$, though the total uncertainty is, on the whole, larger than for other variables. 


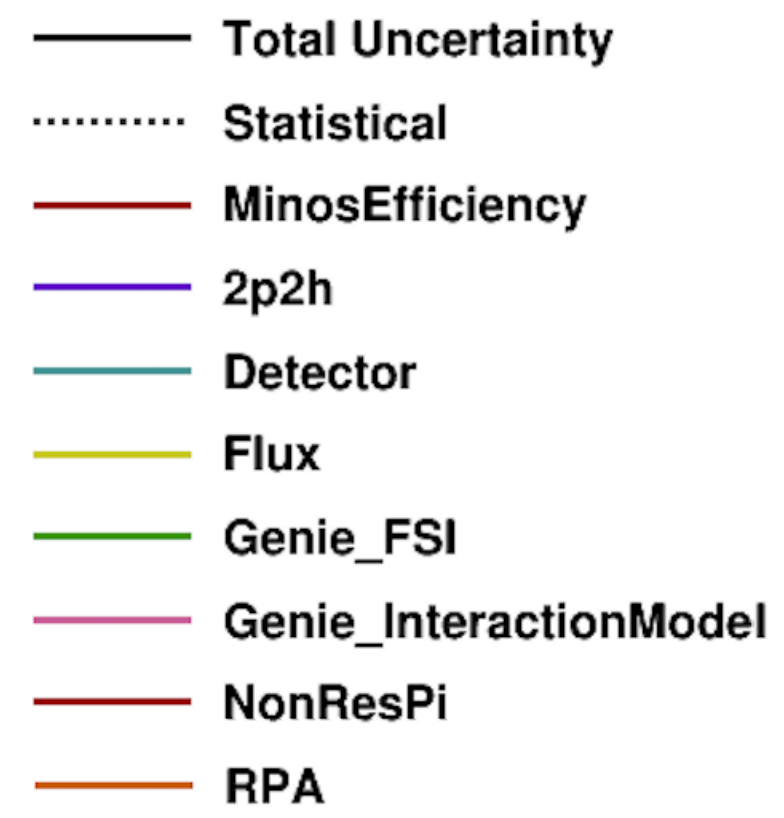

(a) Legend

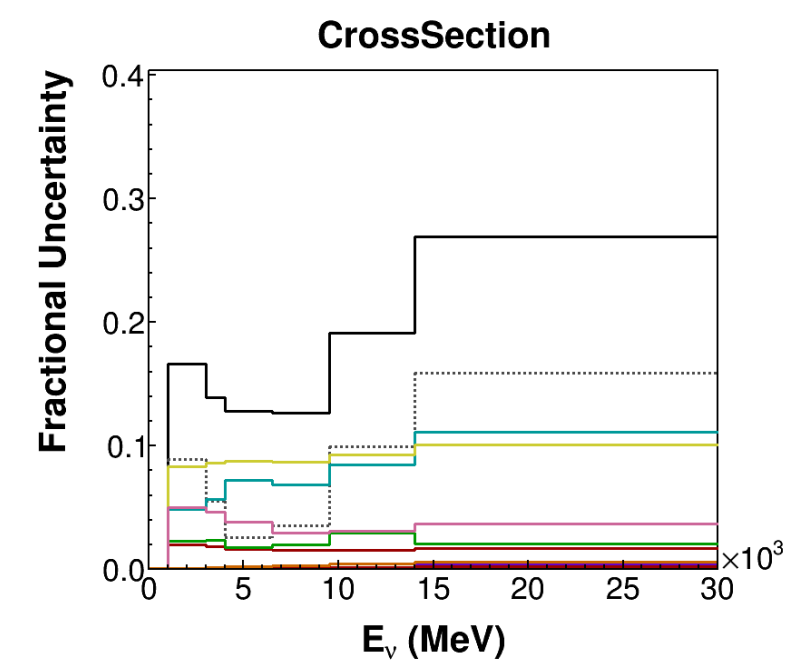

(b) $E_{\nu}$ Cross Section Uncertainties. 


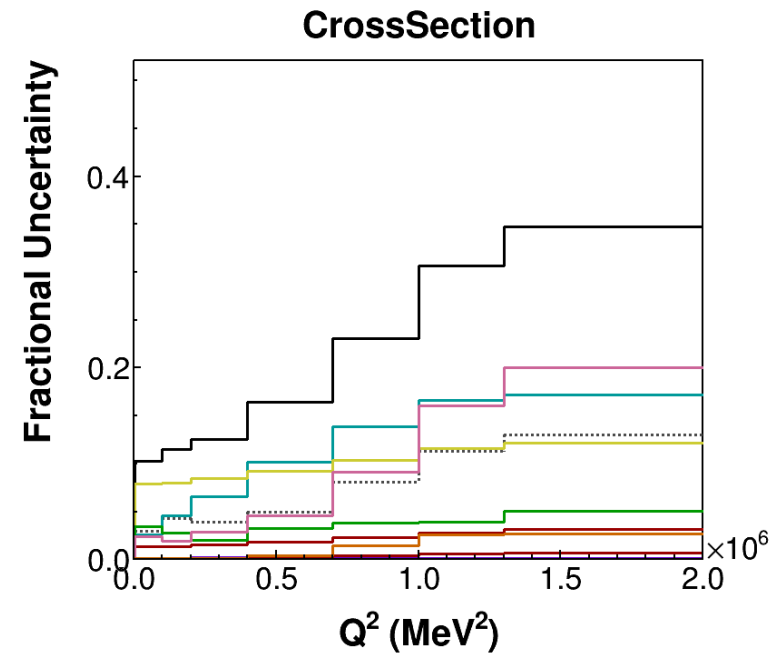

(c) $Q^{2}$ Cross Section Uncertainties.

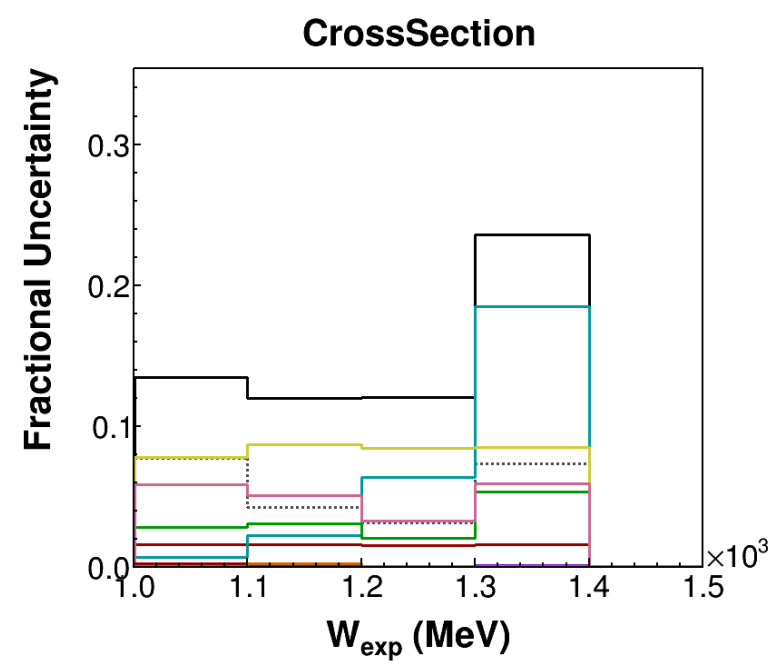

(d) $W_{\text {exp }}$ Cross Section Uncertainties. 


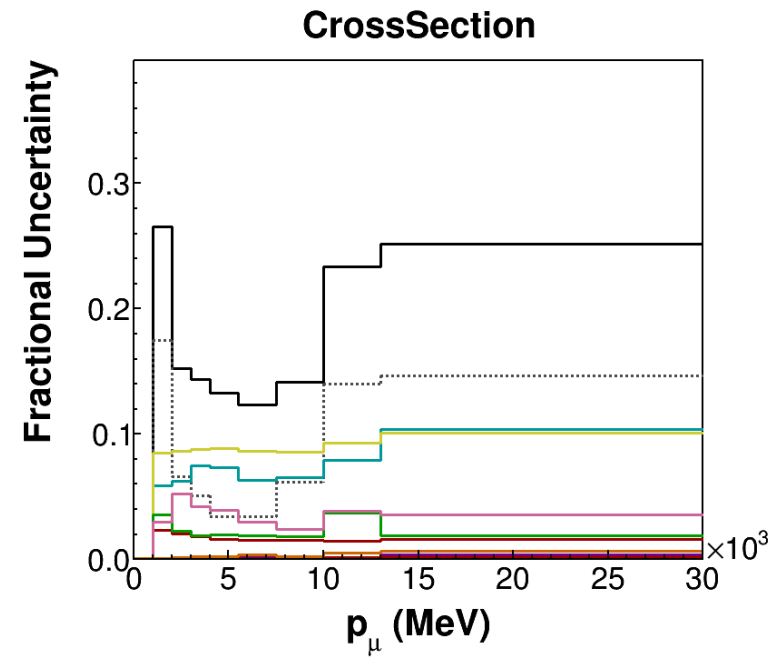

(e) $P_{\mu}$ Cross Section Uncertainties.

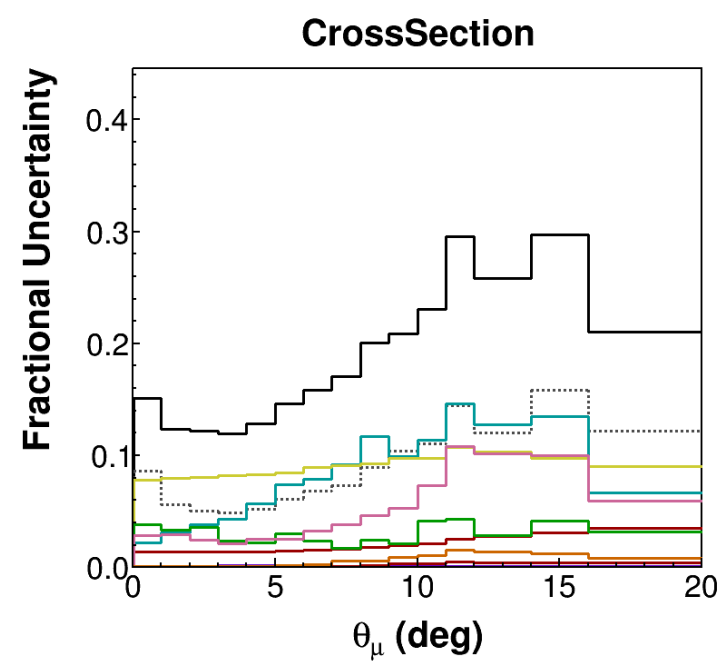

(f) $\theta_{\mu}$ Cross Section Uncertainties. 


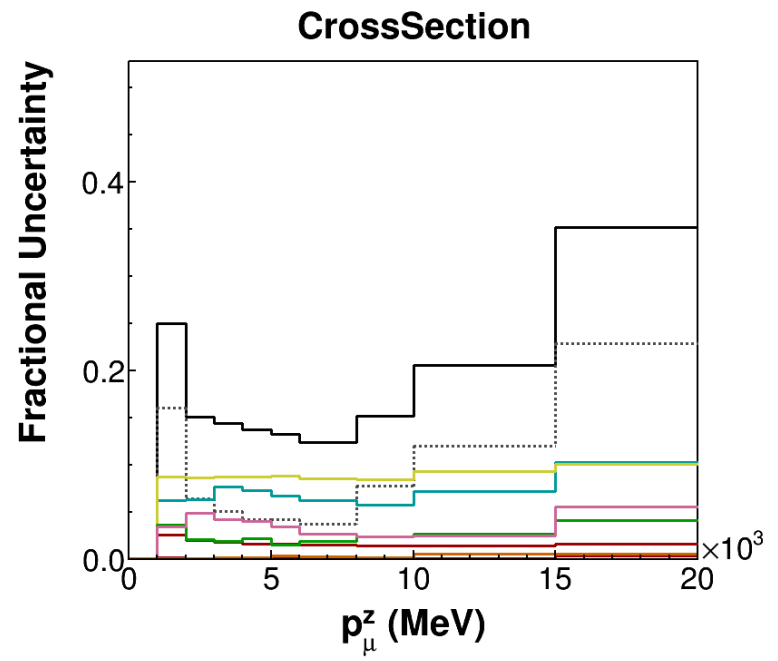

(g) $P_{\mu}^{z}$ Cross Section Uncertainties.

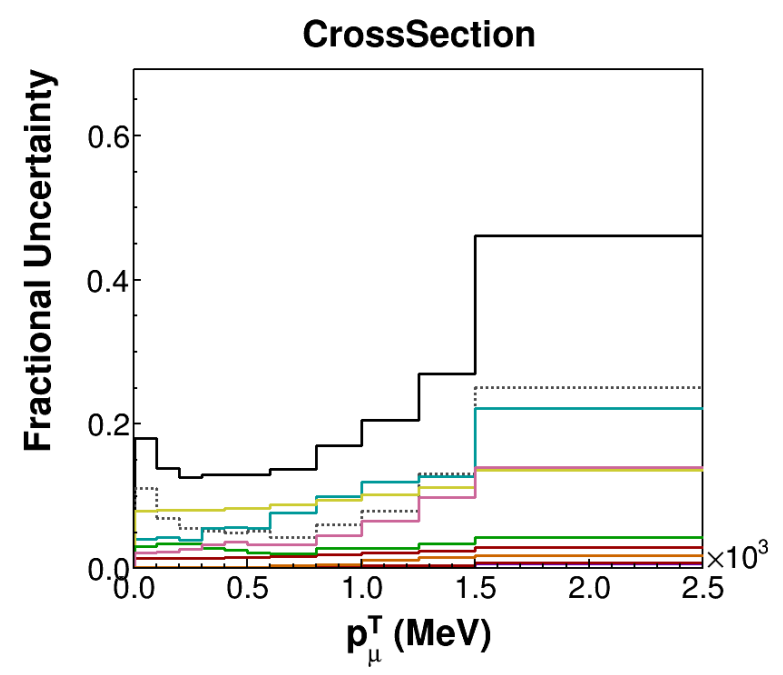

(h) $P_{\mu}^{t}$ Cross Section Uncertainties. 


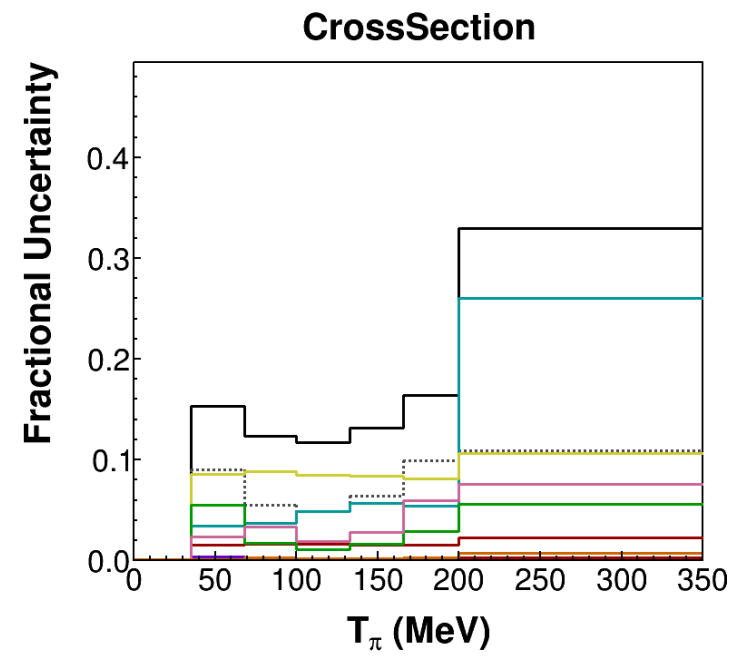

(i) $T_{\pi}$ Cross Section Uncertainties.

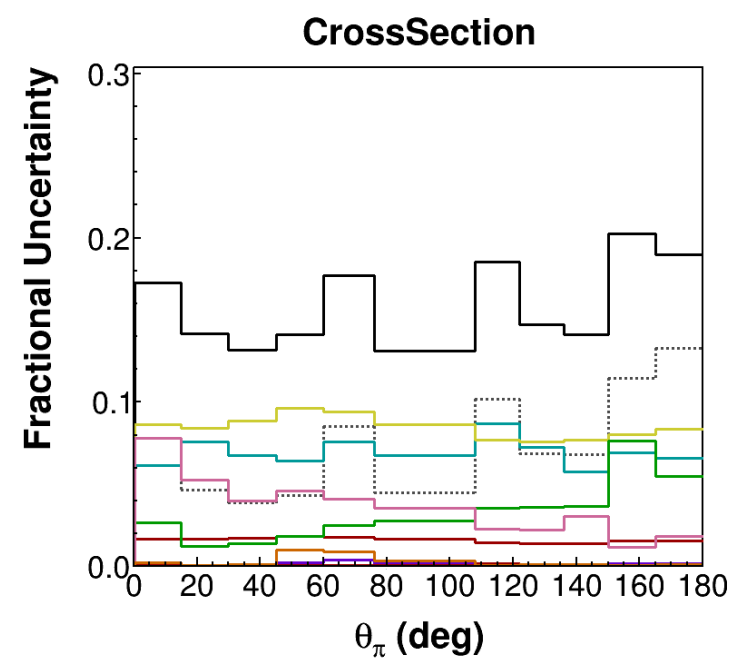

(j) $\theta_{\pi}$ Cross Section Uncertainties.

Figure 7.3: Cross section systematic uncertainties. 


\subsection{RESULTS}

The final cross section results, compared to the MC prediction with MnvGENIE v1 are shown in Figure 8.4.

Generally good agreement is observed across the variables. The lepton variables show generally better agreement than the hadron and event-wide variables.

$E_{\nu}$ and $P_{\mu}$ are closely related variables and show good agreement with the model. $E_{\nu}$ however is significantly more reliant on models, in particular through the simulation of neutral energy and particle response via the recoil energy $E_{h a d}$. Agreement in $\theta_{\mu}$ is mostly good, but with some overprediction for highly forward muons.

The four-momentum transfer squared $Q^{2}$ shows good agreement in all but the first bin, where the model overpredicts relative to the data. This disagreement is well-known and seen in all pion interaction channels [5]. The disagreement is larger in $\pi^{0}$ production (Figure 8.1), which may be explained by coherent pion contribution "filling in" in the discrepancy[31][94]. The $\pi^{0}$ channels also see a turnover in data, which is not seen in charged pion production. This may be due to a diffractive scattering process, in which the neutrino manages to scatter off a free nucleon instead of the whole nucleus. Additionally, there is evidence to suggest that GENIE overpredicts non-resonant contributions to pion production [64][8], and this could also explain the model excess at low- $Q^{2}$, where non-resonant processes most relevant.

Agreement is poor in the experimental invariant hadronic mass $W_{\text {exp }}$; GENIE underpredicts in the rising edge, and overpredicts in the falling edge. $W_{\text {exp }}$ is heavily sensitive to $E_{\text {had }}$, which is difficult to measure. Improvements to $E_{\text {had }}$ measurement are currently being developed. Aside from experimental difficulties, the rising edge of $W$ is where non-resonant processes are expected to interfere most with resonance production. More sophisticted nonresonant models from [68] may be able to shed light on this disagreement. Another possible 


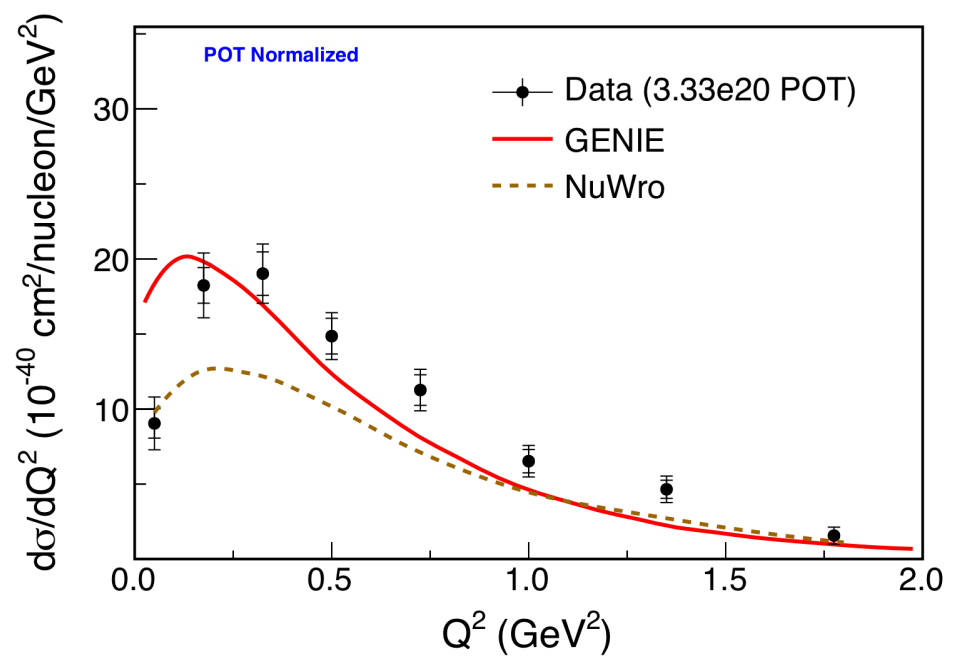

Figure 8.1: MINERvA $\nu_{\mu} \mathrm{CC} \pi^{0}$ production cross section in $Q^{2}$ - in the low energy NuMI datasets. Figure from [24].

explanation for the disagreement is that nuclear effects are under-modeled in GENIE, and, if amplified, could flatten the $W_{\text {exp }}$ distribution to more closely resemble the data.

Agreement in pion variables is worse than in muon variables. The trend of underprediction at low $T_{\pi}$ and overprediction at high $T_{\pi}$ were seen in previous measurements [44]. Though there is agreement in the middle range between $\sim 75-150 \mathrm{MeV}$. In $\theta_{\pi}$ strong disagreement is seen in the 70-110 GeV region, where MINERvA has very little acceptance for pions. As mentioned in Section 6.3.6, a signal definition constraint should be applied to restrict this region.

Figure 8.2 shows the cross section in pion kinetic energy overlaid with the previous MINERvA result [41][44] from the LE NuMI beam configuration. The ME result features 10 times higher statistics (20,000 selected signal events, instead of approximately 2,000 signal events) and reduced errors. The ME beam, with $E_{\nu}$ peak around $7 \mathrm{GeV}$, will access more DIS and less resonance processes compared to the LE beam with $E_{\nu}$ peak around $3.5 \mathrm{GeV}$. Insofar as the measurements disagree (e.g. the first and last bins) the explanation could be due to bias by relatively mismodeled DIS or resonance processes. Results from T2K [3] also 
show little turnover at low pion momentum.

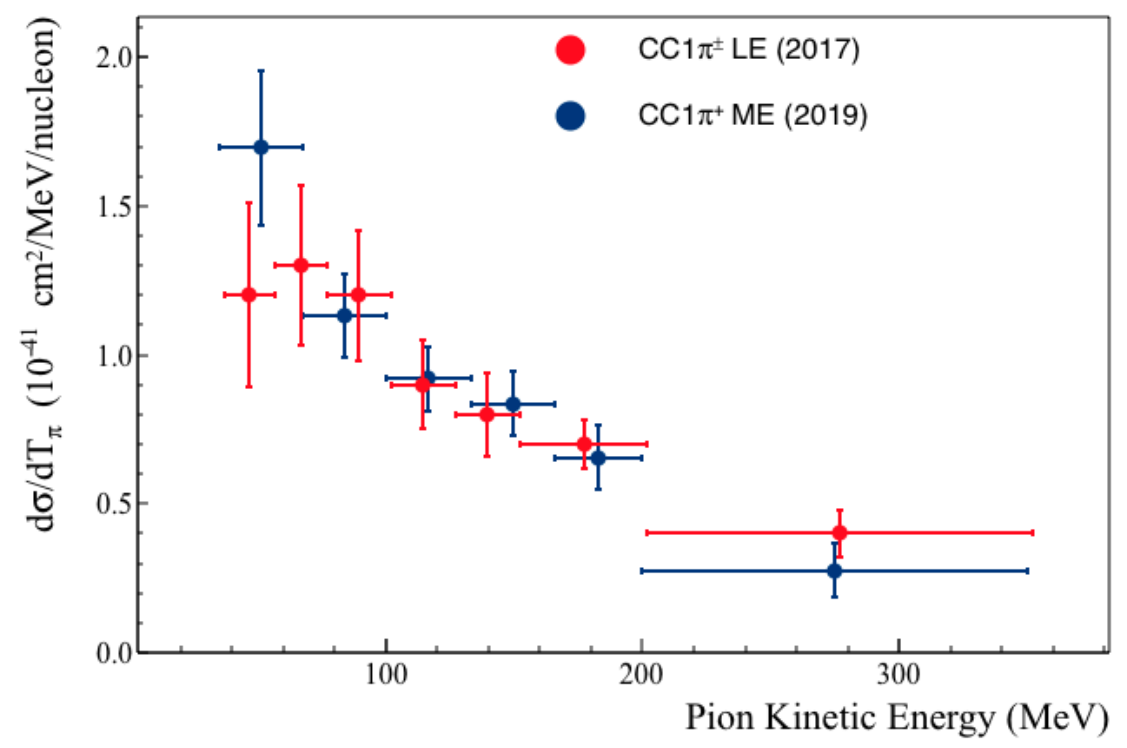

Figure 8.2: MINERvA pion production cross sections in $T_{\pi}$ - in the LE and ME datasets.

Figure 8.3 shows the sample as a function of $T_{\pi}$ at the event selection stage and broken down by interaction channel and multiplicity. From these Figures it can be seen that it is primarily $\mathrm{CC} 1 \pi^{+}$resonance that is being selected. During efficiency correction, other non-resonant, signal processes may be replaced in the sample. 


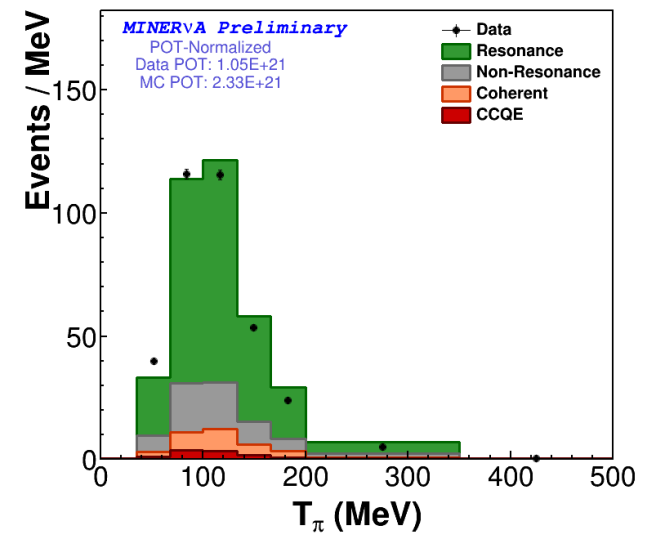

(a)

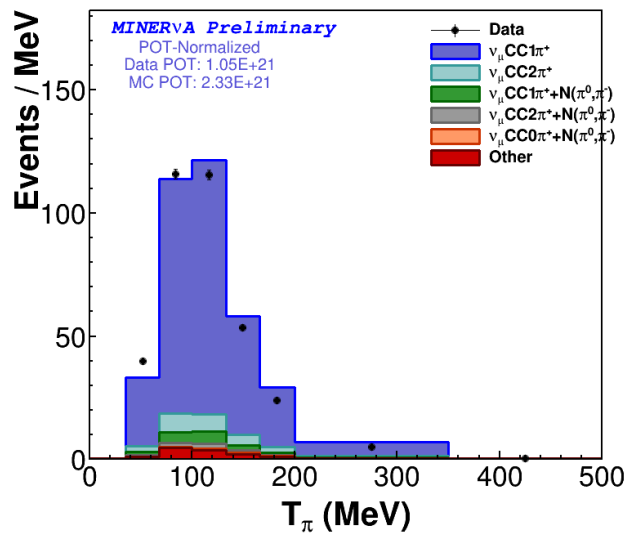

(b)

Figure 8.3: Event selection in $T_{\pi}$, broken down by (left) interaction channel and (right) pion multiplicity. Includes both signal and background. The non-resonant component (gray) includes both DIS and true non-resonant, background pion production events. 


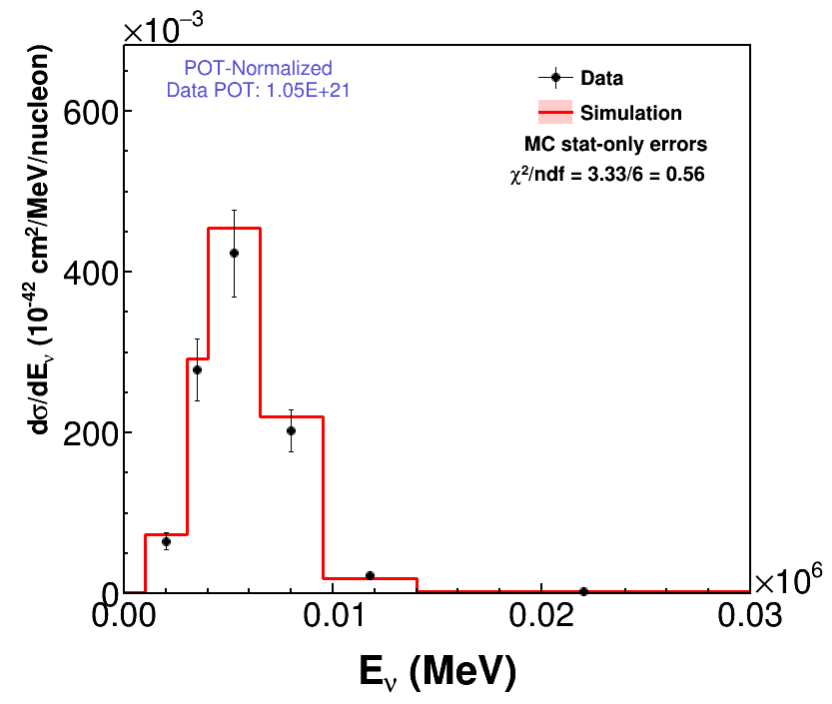

(a) $E_{\nu}$ cross section

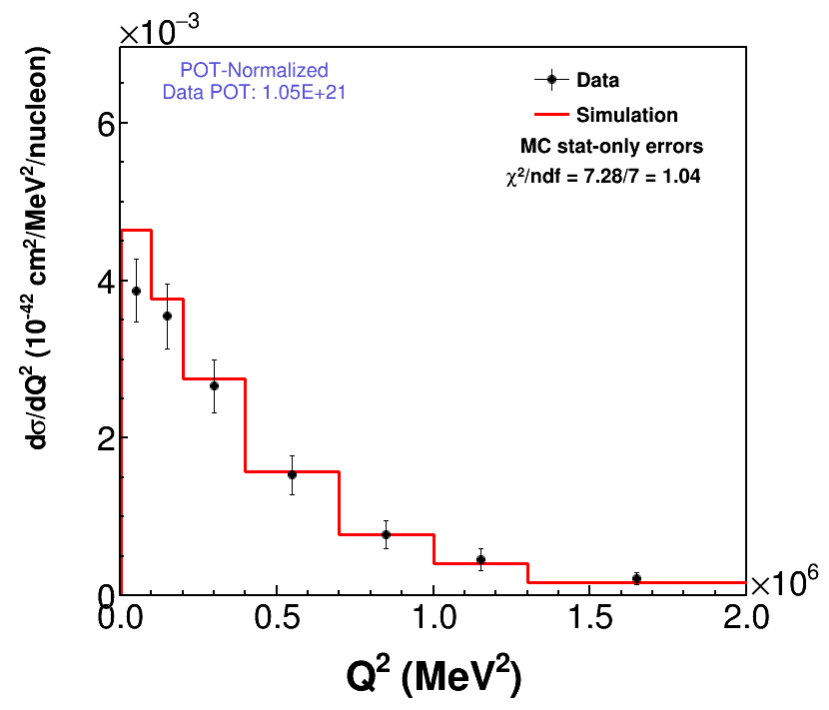

(b) $Q^{2}$ cross section 


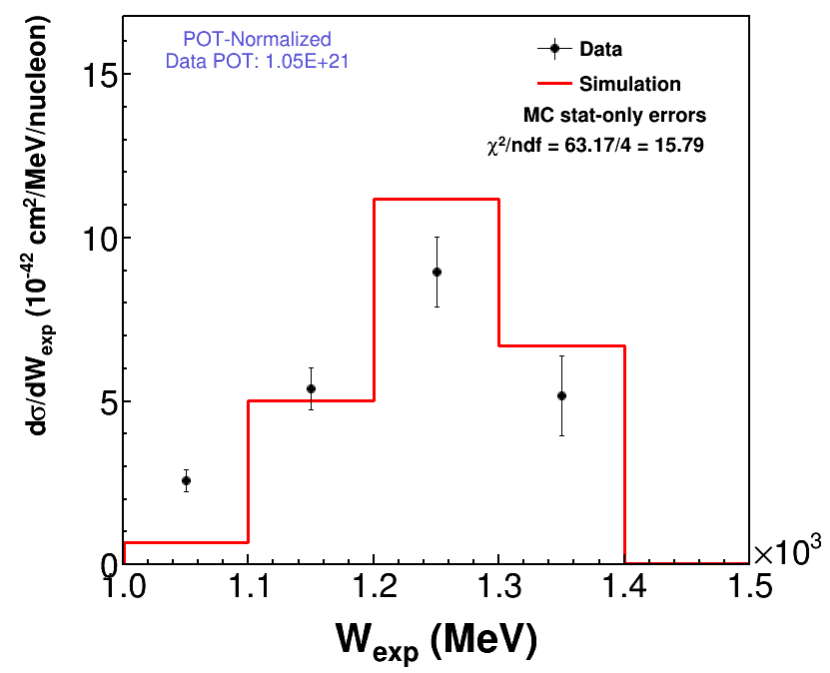

(c) $W_{\text {exp }}$ cross section

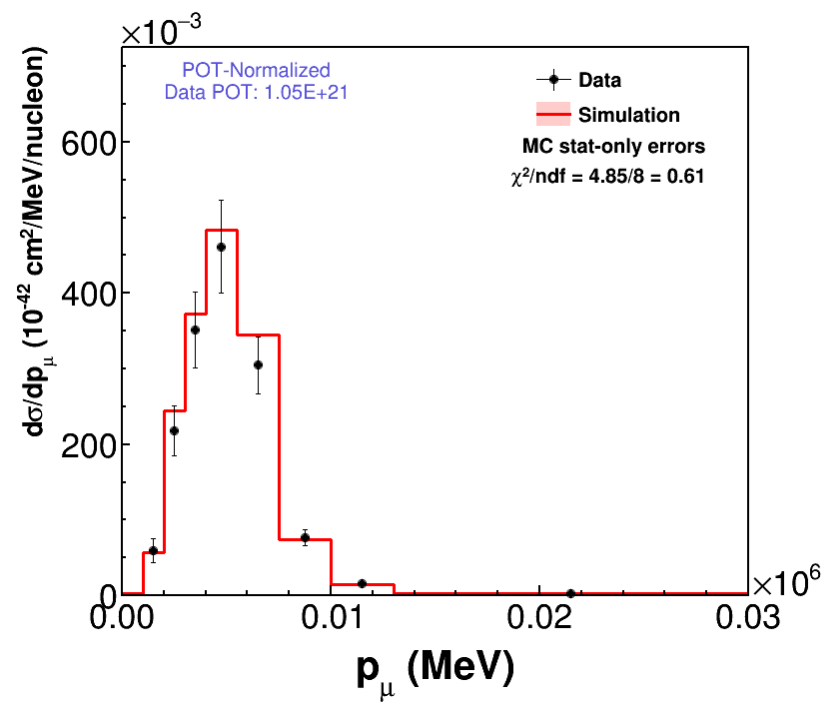

(d) $P_{\mu}$ cross section 


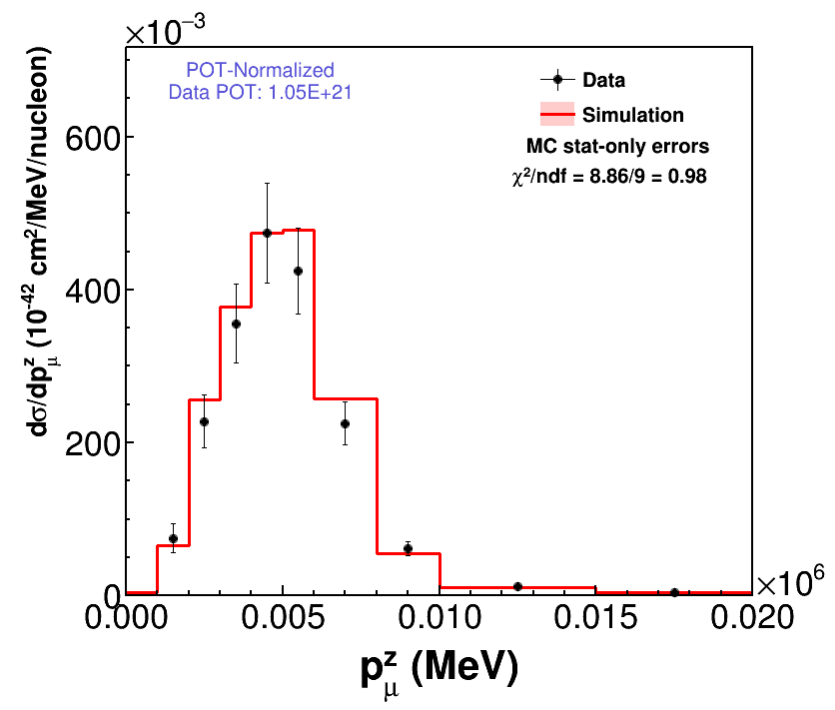

(e) $P_{\mu}^{z}$ cross section

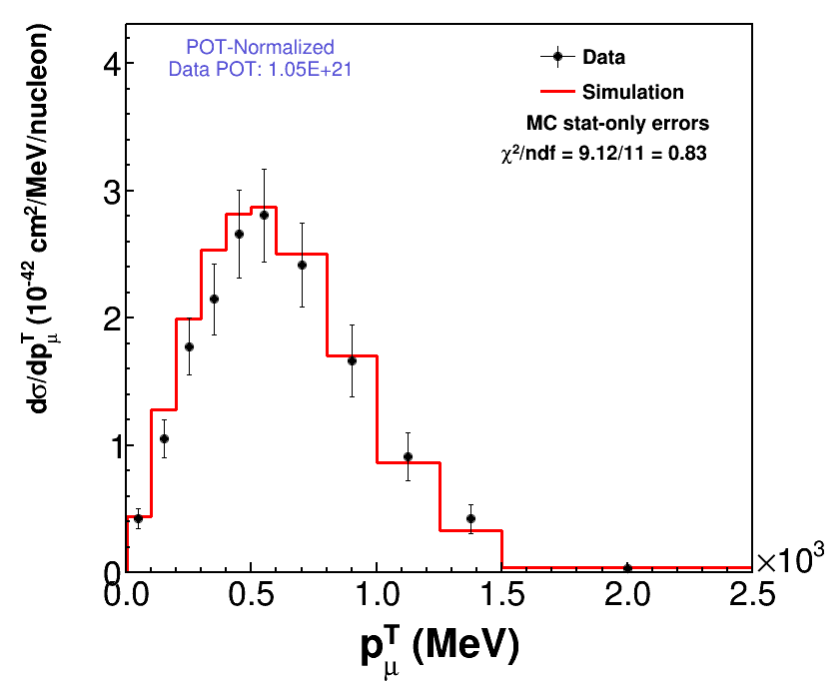

(f) $P_{\mu}^{t}$ cross section 


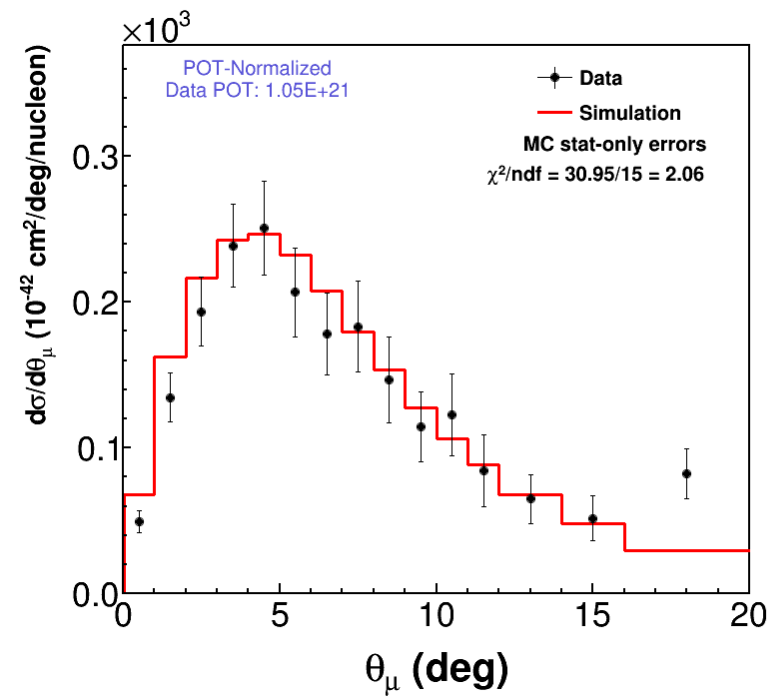

(g) $\theta_{\mu}$ cross section

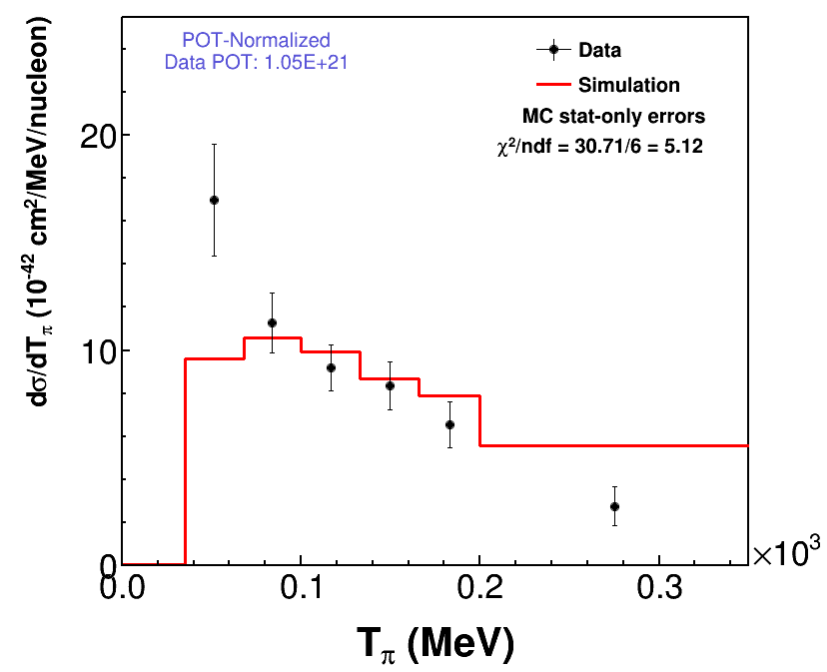

(h) $T_{\pi}$ cross section 


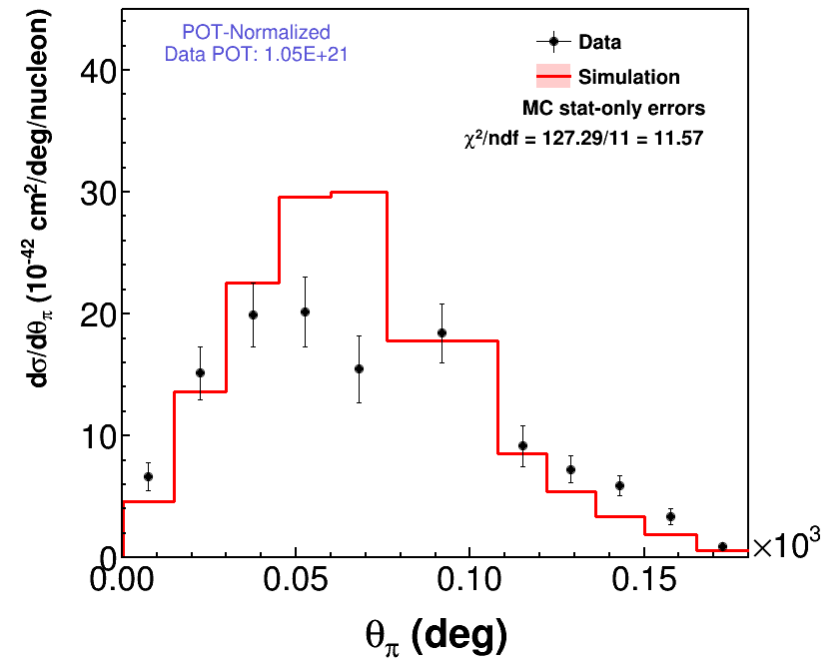

(i) $\theta_{\pi}$ cross section

Figure 8.4: Cross section results as a function of analysis variables. 


\subsection{CONCLUSION}

The success of the next-generation long baseline neutrino oscillation experiments requires well-understood neutrino flux and interaction models. In the operating energy range of these experiments, pion production is a dominant interaction channel, and several effects related to neutrino scattering with a heavy nucleus to produce a pion are still mis- or un-modeled.

As the primary result, this thesis has presented a measurement of muon neutrino-induced charged current charged pion production. Differential cross sections are measured as a function of muon and pion kinematics, neutrino energy, four-momentum transfer squared, and, for the first time, as a function of experimental invariant hadronic mass. Care was taken to minimize model dependence in the measurement, which make the results maximally useful to theorists, model builders, and other experiments who wish to make comparisons.

Results are compared to predictions from the GENIE event generator and show generally good agreement. Agreement in the lepton variables is somewhat better than in hadron and event-wide variables. Disagreements are observed at low- $Q^{2}$, which are suggestive of certain unmodeled or mismodeled nuclear effects. Disagreement in pion kinetic energy have been previously seen in measurements of this channel. In experimental invariant hadronic mass GENIE underpredicts at the rising edge of the resonance peak and overpredicts at the falling edge. These disagreements could indicate undermodeled nuclear effects or more complex non-resonance interferences, which are expected to shift the position of the resonance peak.

Additionally, developments in neutrino beam modeling are presented in Appendix A. A critical distinction was drawn between axially symmetric and asymmetric focusing effects, and it was observed that many longitudinal focusing parameter shifts are realized as similar effects on the flux focusing peak. 


\section{APPENDIX A. FLUX MODEL FOCUSING UNCERTAINTIES AND STUDY OF FLUX SIMULATION DISCREPANCY}

This appendix discusses in more detail the adjustable parameters of the flux focusing system model and the effect they have on the flux prediction. Work in this area was performed in parallel with the cross section analysis described in Chapter 6 in order to calculate Medium Energy (ME) era focusing uncertainties for all MINERvA cross section measurements (A.1) as well as to search for the cause of a data-MC discrepancy that MINERvA observes in the flux (A.2).

\section{A.1 MEDIUM ENERGY-ERA FLUX FOCUSING UNCERTAINTIES}

Several improvements were made on the Low Energy focusing uncertainty calculation. In the LE configuration, the target-horn separation was minimzed and flux was less sensitive to the focusing system. In the ME configuration, by contrast, the target-horn separation was large, whether hadrons were or were not focused was much more sensitive to focusing parameters. The LE focusing uncertainties are shown in Figure A.1, and the ME focusing uncertainties are shown in A.2. 


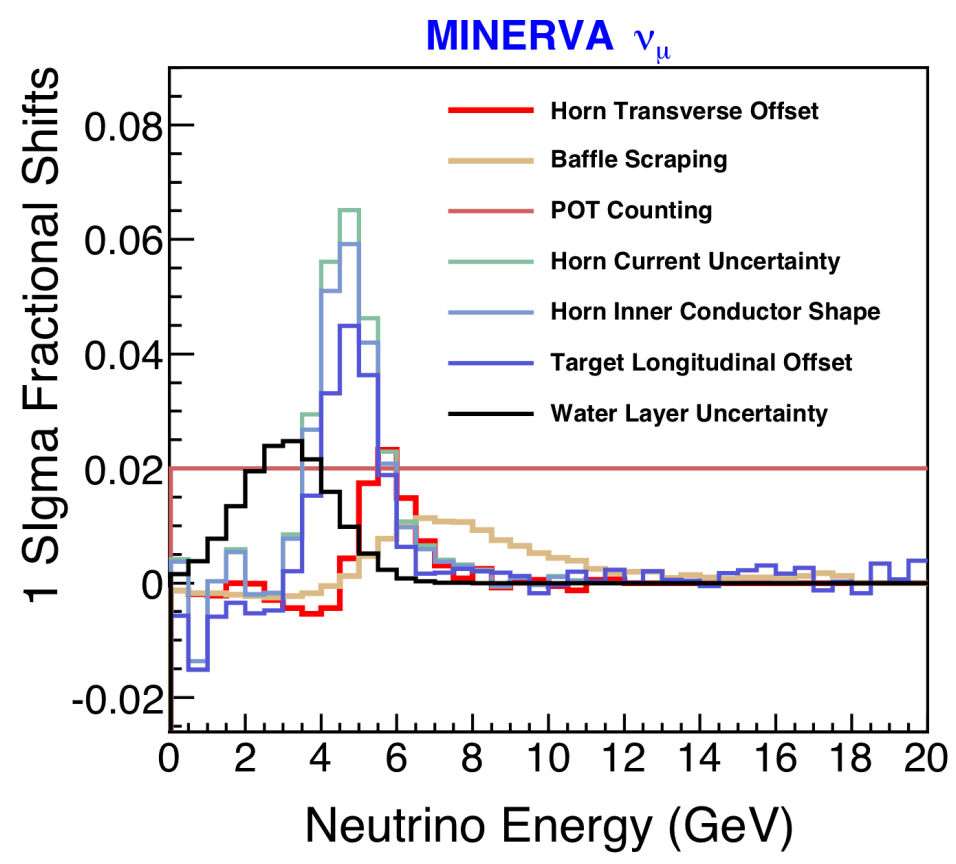

Figure A.1: Low Energy-era focusing uncertainties. Note that the LE flux was less sensitive to the focusing system (compare with Figure A.2).

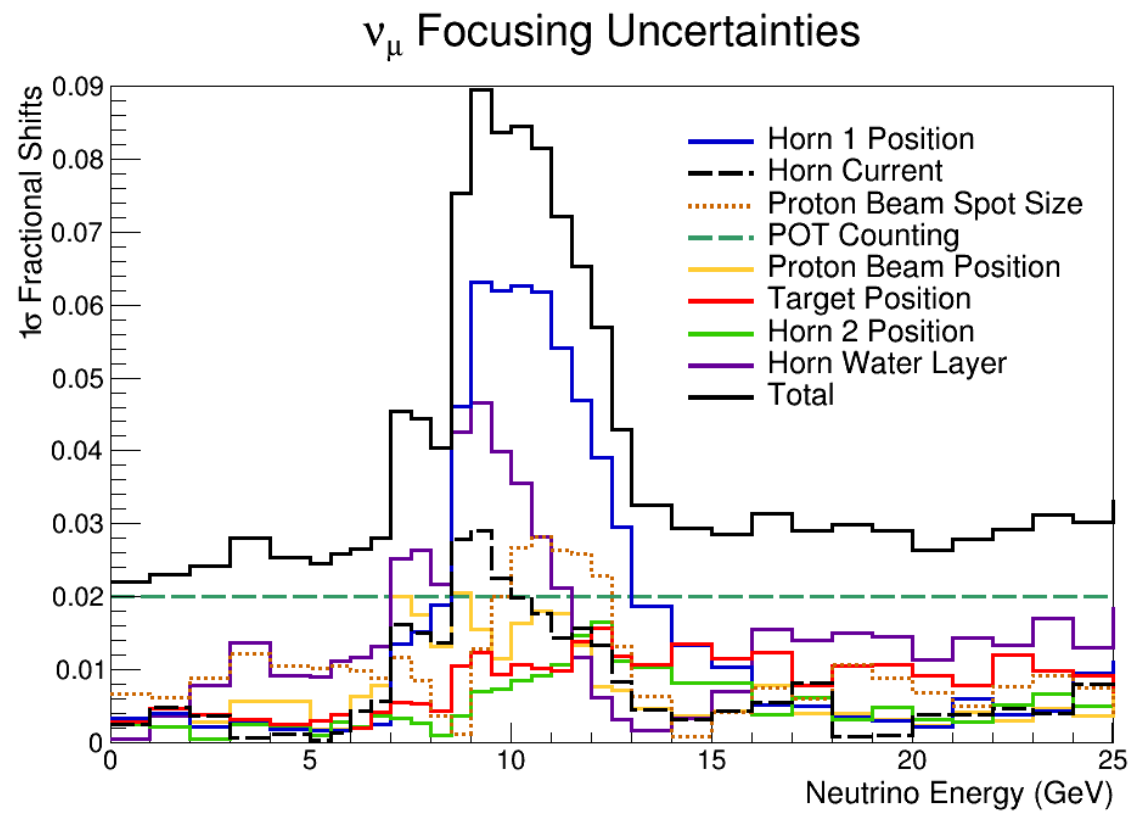

Figure A.2: Medium energy focusing uncertainties. 


\section{A.1.1 General improvements to the flux model and calculation}

First, general improvements to G4NuMI and the flux extraction process include:

- Corrected MINERvA position relative to the beam center The low energy flux uncertainties were calculated using a MINERvA position that was $\sim 30 \mathrm{~cm}$ further from the beam center than its actual value.[86]

- Flux calculated from neutrinos throughout the MINERvA detector volume In the LE, the focusing uncertainties were calculated via the flux estimation at a single $\mathrm{x}-\mathrm{y}-\mathrm{z}$ point located at roughly the center of the MINERvA volume. This was updated to calculate the flux from neutrinos throughout the entire MINERvA volume. The total effect of the corrected MINERvA center position and of the integrated flux calculation was as high as an $8 \%$ change in the uncertainties for parameters that shift a focusing component asymmetrically in a direction transverse to the beam. In contrast parameters that shift a focusing component parallel to the beam direction or which perform an azimuthally symmetric shift are not so-affected.

- Flux calculation improvements The flux calculation procedure was migrated to a parallelized grid computing method to increase speed. A reweighting scheme to increase statistics without increasing storage space by re-using neutrino parents was also implemented [91].

\section{A.1.2 Focusing parameter tolerance refinements}

The tolerances of several focusing parameters were refined, the uncertainties for some of which were determined to be negligible and removed, and other focusing parameters were added.

A.1.2.1 Proton beam spot size The cross sectional area, or spot size, of the incident proton beam is roughly gaussian with measured $1 / e$ horizontal and vertical widths measured in $\mathrm{mm}$. The spot size was found to be dependent on the proton beam intensity (see Figure A.3), which increased over the course of the ME run. Figure A.4 shows the increase in spot size over a three year period during the ME. 


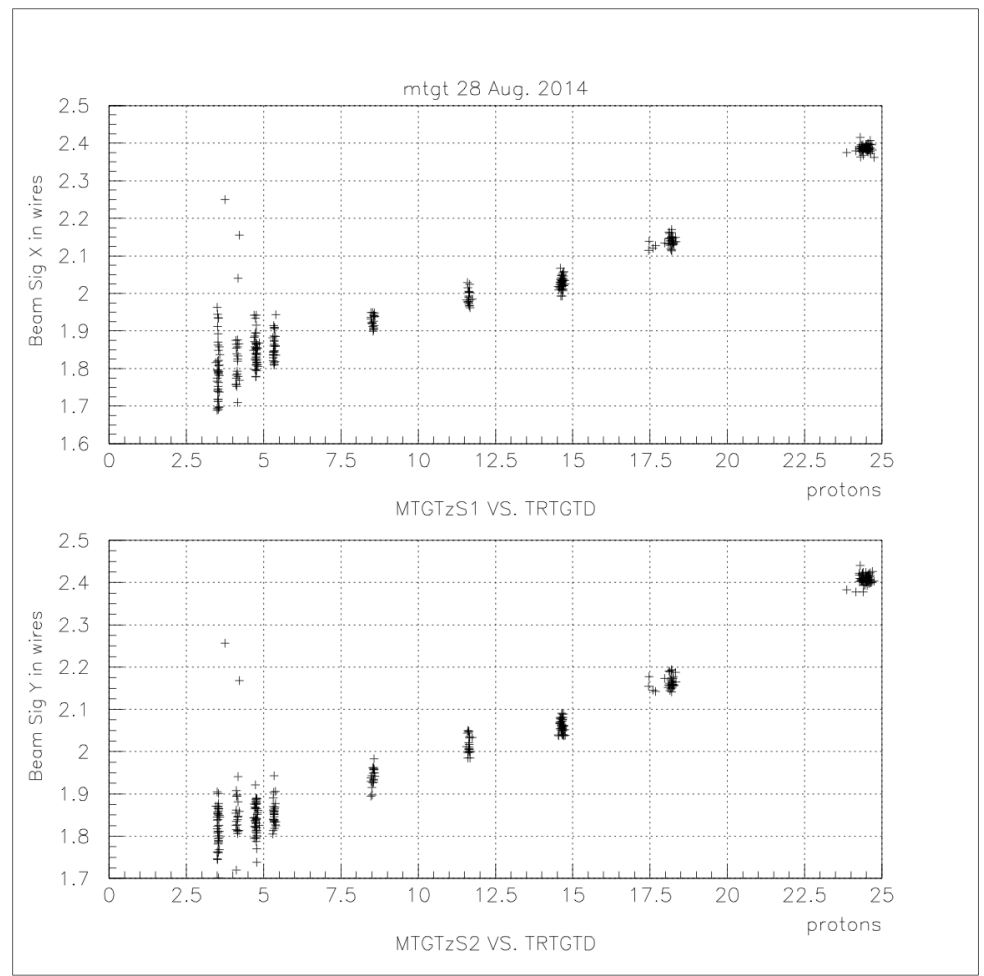

Figure A.3: Measured in wire widths of $0.5 \mathrm{~mm}$ figure shows the $1 / \mathrm{e}$ horizontal and vertical sizes of the proton beam as a function of intensity (e12). A linear fit to the data gives $\sigma=0.4 *$ intensity +1.45 which is reliable throughout the ME. Figure courtesy of D. Jensen. 


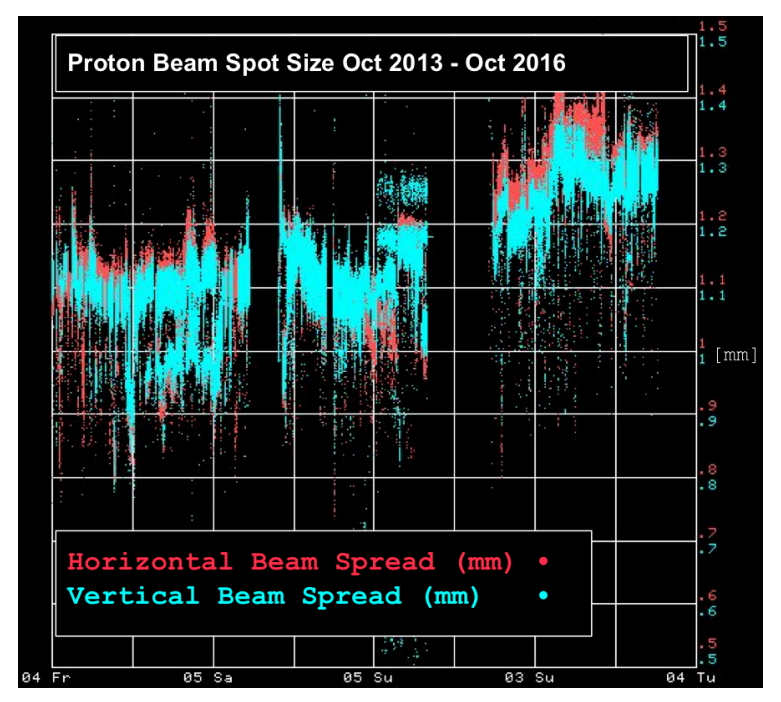

Figure A.4: The proton beam spot size during a three year period of the ME. Shown before a flat calibration factor of 1.08 is applied. Beam spot size is a function of beam intensity, which increased over the course of the ME run.

A flat calibration factor of 1.08 is applied to the values in A.4 to give nominal spot sizes of roughly $1.2 \mathrm{~mm}$ and $1.4 \mathrm{~mm}$ for two periods, corresponding to before and after the September 2015 Shutdown. The MINERvA Monte Carlo dataset was simulated entirely with spot size $1.2 \mathrm{~mm}$, thus MC events after the Fall 2015 Shutdown were assigned a weight corresponding to a flux change up to $1.4 \mathrm{~mm}$ beam spot size.

From a statistical variation of $8 \%$ and a $10 \%$ systematic uncertainty on the calibration constant, the total uncertainty for both periods is taken conservatively to be $\pm 2 \mathrm{~mm}$.

\section{A.1.2.2 Proton beam baffle scraping Surrounding the NuMI target is a cylindrical} baffle of inner radius $6.5 \mathrm{~mm}$. The purpose of the baffle is to protect the downstream target apparatus from errant beam proton damage. Assuming a proton beam gaussian spot size of width $1.2 \mathrm{~mm}, \ll 1 \%$ of protons are expected to collide with the baffle. Nonetheless the non-gaussian-ness of the spot size tail has been estimated twice. In the first attempt[73] the tail was extrapolated from a gaussian fit to a measure of the beam profile. From this 
the fraction of protons scraping the baffle was determined to be $0.15 \%$, and a conservative uncertainty of $0.25 \%$ was used. In the second attempt [88], the baffle scraping was estimated by monitoring its temperature. From that estimate a conservative upper limit of $0.6 \%$ was given. Both estimates were made in the LE era when the beam spot size was larger $(\sim$ $1.5 \mathrm{~mm})$ and the baffle inner radius was smaller $(5.5 \mathrm{~mm})$, the effect of baffle scraping is expected to be smaller in the ME.

To estimate the effect of baffle scraping a flux was simulated with incident proton beam of spot size $0.01 \mathrm{~mm}$ pointed directly into the baffle at radius $13 \mathrm{~mm}$. The flux was then divided by the nominal prediction and scaled by $0.25 \%$. The result was an uncertainty of $<$ $0.5 \%$ across all bins of $E_{\nu}$.

A.1.2.3 Target longitudinal position The LE-era target was designed to move along its z-axis as a tuning method and its uncertainty was (conservatively) set at $1 \mathrm{~cm}$ [19][66]. The ME target position was known much more precisely, to within $1 \mathrm{~mm}$ [88]. The resulting flux uncertainty was found to be $<0.5 \%$ across all bins of $E_{\nu}$.

A.1.2.4 Focusing horn currents In the LE, the horn current tolerance was stated 1\%, but this value was revisited for the ME. From NuMI databases, the horn current was plotted over the for the entire ME (Figure A.5), and a $0.2 \%$ statistical deviation was measured. Combined with a $0.5 \%$ systematic error, a resulting $0.5 \%$ tolerance was determined. The horn current contribution to the focusing uncertainty peaks at $2.5 \%$ between the focusing peak and falling edge.

\section{A.1.2.5 Horn 1 geometry model In the LE, an improved horn 1 inner-conductor} model was implemented. While the old model was used for central value flux production, the improved model was used as an additional focusing uncertainty, giving a $6 \%$ uncertainty between the focusing peak and falling edge.

The improved model implemented a horn 1 geometry model based off of DUNE/LBNE flux simulations. More specifically, the new model switched the geometry objects modeling the horn from G4PolyCones to G4Cone, and it doubled longitudinal segmentation. The 


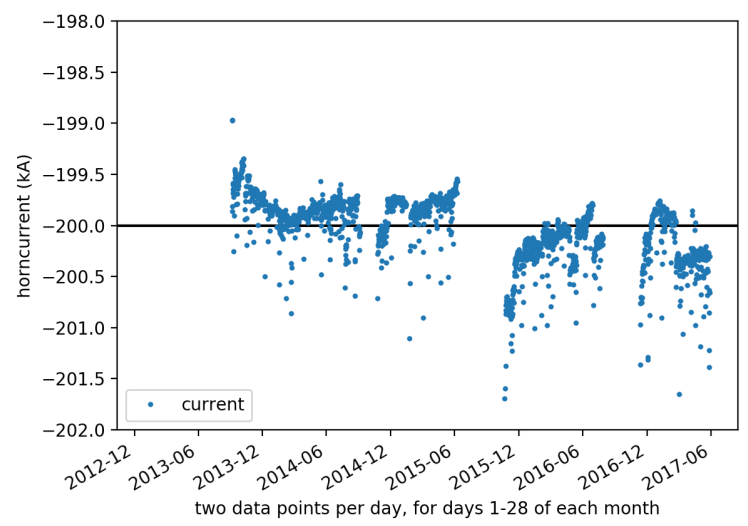

Figure A.5: Horn current from NuMI database during the ME run.

largest changes in the neutrino parent hadron interation positions due to the new model were observed in the downstream end of the inner-outer conductor transition, I/O spider supports, and I/O welds [78].

The improved model is used as default in the ME simulation. Two uncertainties due to the new model were considered: one from the comparison to the old model and a second from doubling the segmentation granularity of the new model's G4Cones. The flux of the more-finely segmented model differed from that of the nominal segmentation by $\sim 1 \%$, and the uncertainty was removed from consideration. The uncertainty from the old model was also removed because it is believed that the new model is strictly more accurate and because the comparison to the old model captured no current uncertainty on the model.

\section{A.1.3 Focusing uncertainties by category}

The full list of list of focusing uncertainties considered is given in Table 7.4. The focusing uncertainties are shown in A.6.

On top of a $2 \%$ POT-counting pedestal, focusing effects tend to pile up at the focusing peak and falling edge from $7 \mathrm{GeV}<E_{\nu} 12 \mathrm{GeV}$. 

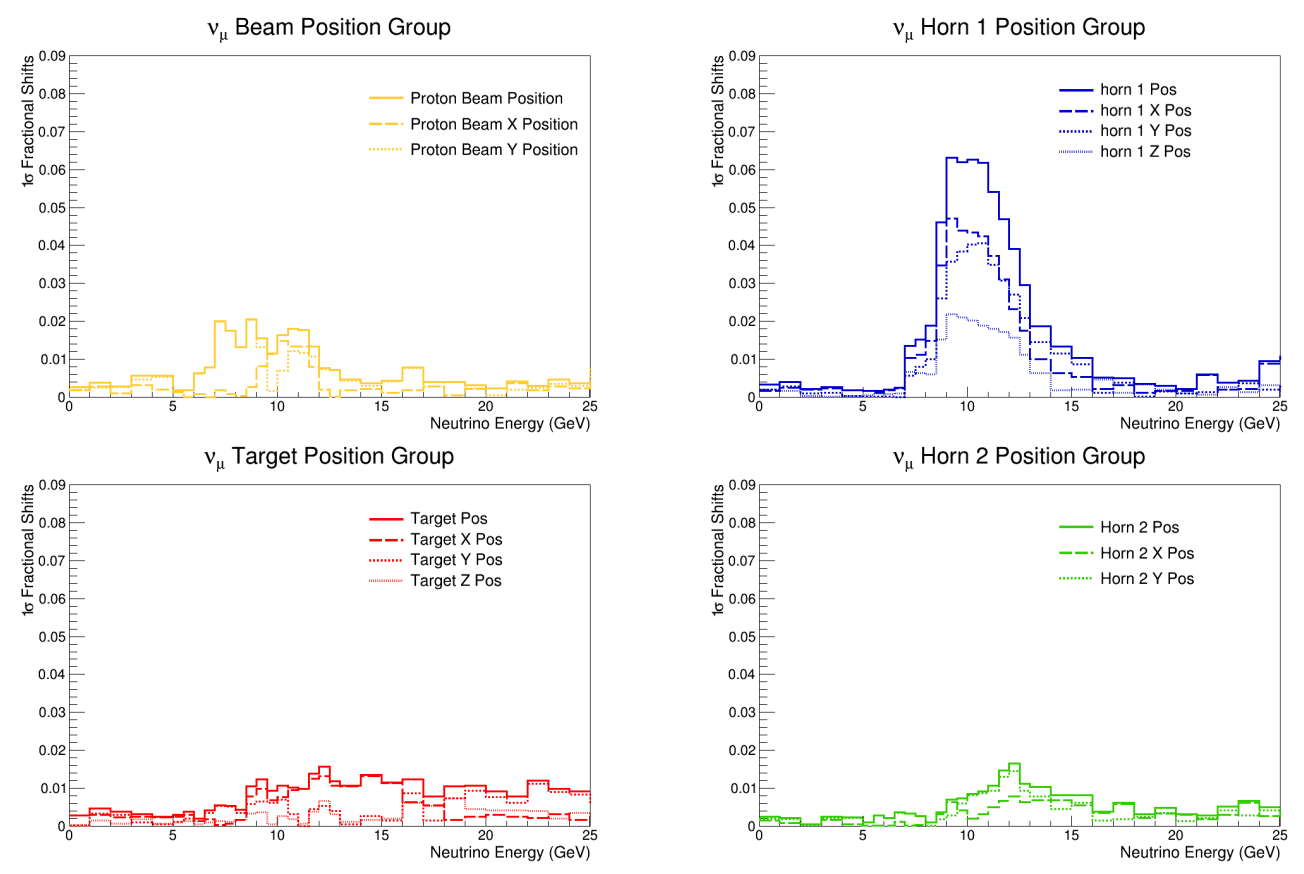

Figure A.6: Breakdowns of beam, target, and horn uncertainties from Figure A.2.

\section{A.2 DATA-MONTE CARLO FLUX DISCREPANCY IN MINERVA}

The most direct data measurement of the flux is through the standard candle process referred to as charged current low-recoil $(\nu)$ inclusive scattering [107]. Measurements of this process in MINERvA during the medium energy reveal a large data-MC disagreement, shown in Figure A.7, suggesting a problem with flux model.

Within the flux model a problem could exist in the neutrino parent hadron production model or in the focusing system model. To search for a cause of the discrepancy, fits were performed in which hadron production or focusing parameters were floated to shift MC and obtain a data-MC match. Two independent methods [65][29] determined that the hadron production parameters were not sensitive to the shape of the discrepancy. Additionally, in two data samples that are less sensitive to the focusing system, viz. the zero horn current and antineutrino forward horn current samples, the discrepancy lessened or disappeared [108][109]. 


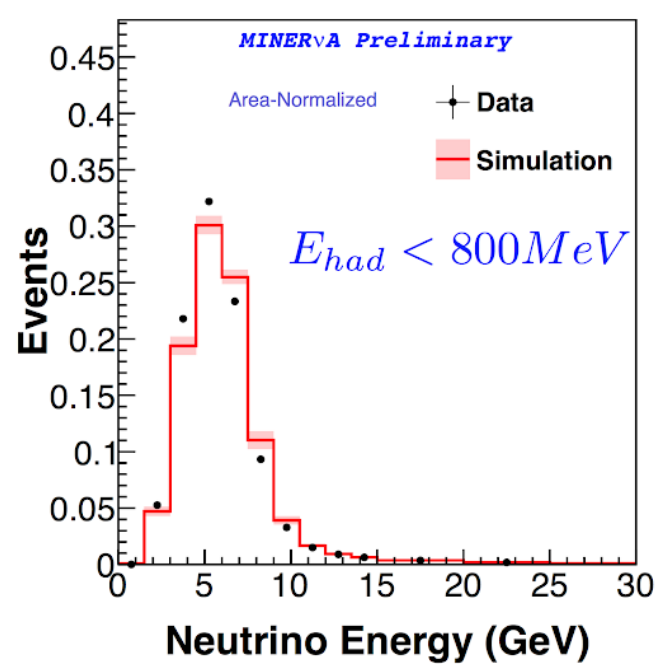

(a)

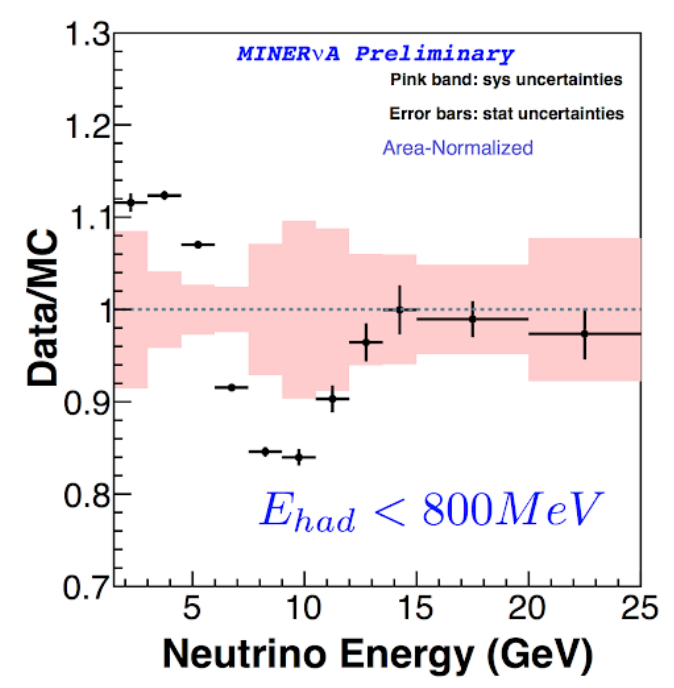

(b)

Figure A.7: MINERvA observes a data-MC discrepancy in the low-recoil energy inclusive event rate, a proxy for the flux.

Consequently, a study of the focusing system was launched, looking for a cause of the low- $\nu$ event rate discrepancy.

Several methods, metrics, and visualization tools were used or invented to better understand the focusing system - to gain intuition and search for a cause of the discrepancy, either among the known focusing system parameters or somehow unkown and implicit in the model. In the former case, the problem could be caused by something as simple as, for example, a mistake in the NuMI beamline surveying of a horn position or a misimplementation of the surveyed value in the simulation. In the latter case, the problem could be an unacceptable simulated boundary condition on the horn magnetic field, or a excessively large or small stepping constant defining how finely the magnetic field is segmented. 


\section{A.2.1 Neutrino interaction position heatmaps}

First, it was not known how well the data and MC agreed on the neutrino position distribution across the face of the detector. Simulation heat maps of the neutrino interaction position (Figure A.8) reveal interesting shapes but no obviously unphysical behavior. Recall that the NuMI beam centroid is offset from the MINERvA center by approximately $25 \mathrm{~cm}$ in the horizontal direction and $25 \mathrm{~cm}$ in the vertical direction (refer to Figure 4.7). Heat maps were separated into both bins of neutrino energy as well as longitudinal interaction position. No unexpected shapes arose which warranted comparision to data. Observe the flux peak at the 6-7 and 7-8 GeV bins, the flux dip surrounding the beam centroid in the 4-5 GeV bin, and the shadow of the target mounting at bottoms of the 10-12 and 12-14 GeV bins. 


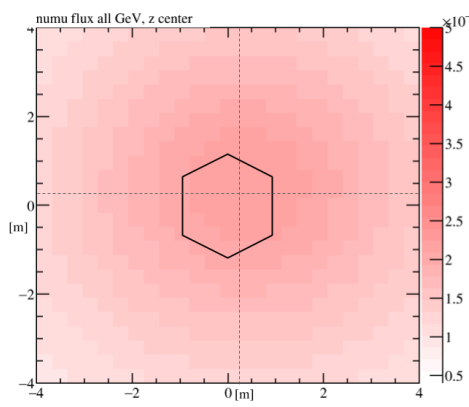

(a) All neutrino energies

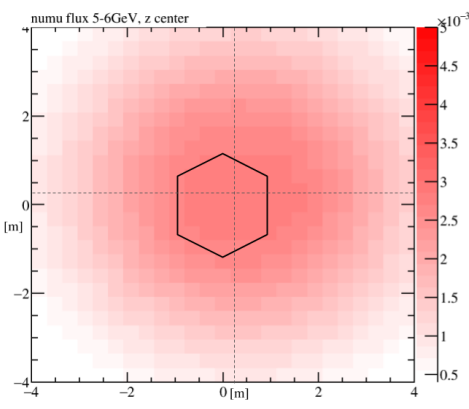

(c) $5-6 \mathrm{GeV}$

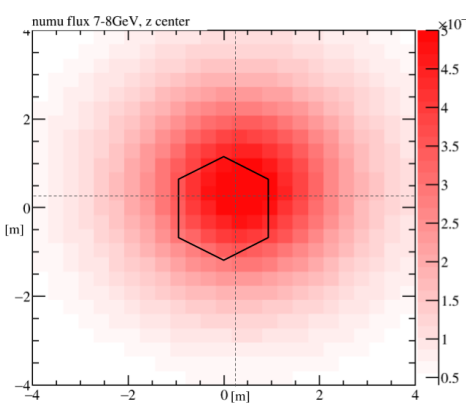

(e) $7-8 \mathrm{GeV}$

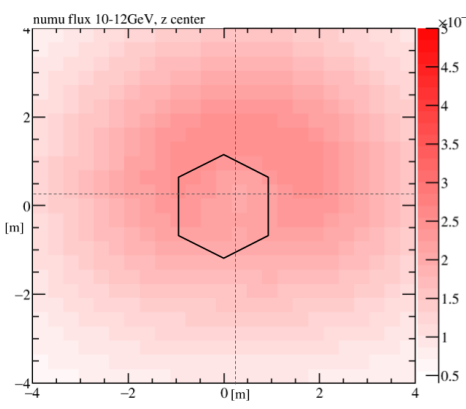

(g) $10-12 \mathrm{GeV}$

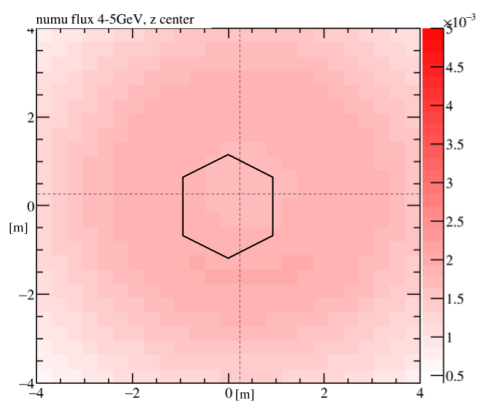

(b) $4-5 \mathrm{GeV}$

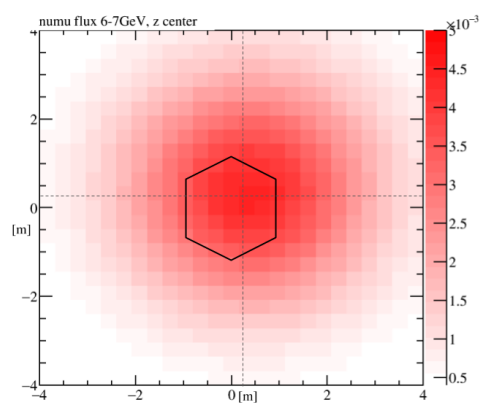

(d) $6-7 \mathrm{GeV}$

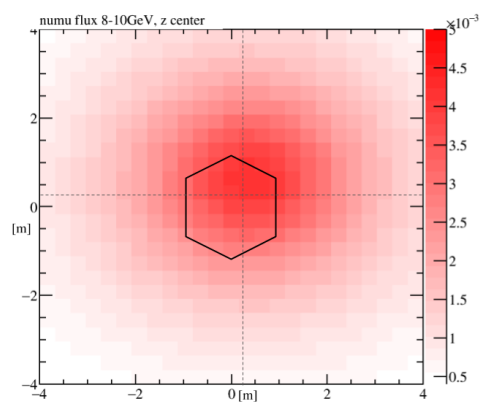

(f) $8-10 \mathrm{GeV}$

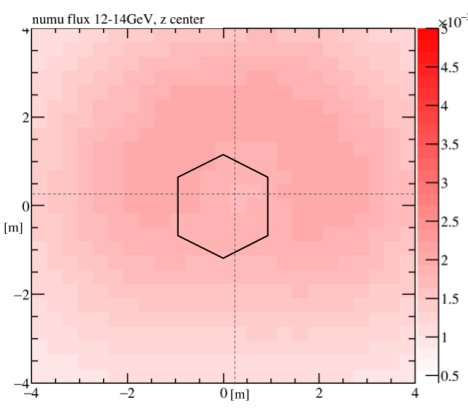

(h) $12-14 \mathrm{GeV}$

Figure A.8: Neutrino interactions by energy at the MINERvA z-center with the approximate detector face overlay and beam centroid. 


\section{A.2.2 Neutrino mean interaction position}

Plots of the mean neutrino interaction $\mathrm{x}-\mathrm{y}$ vertex position complement the heat maps. A "spiral" shape is observed as the mean interaction point is traced across increasing neutrino energy bins. Figure A.9 compares the mean neutrino interaction position for the nominal flux prediction to that of the flux from shifted focusing parameters. Note that because MINERvA is not centered on the beam, shifts perpendicular to the beam direction distort the beam shape more than shifts of a parameter parallel to the beam. The mean interaction positions of some energy bins are strongly moved by focusing parameter shifts while others are unaffected. A similar sprial pattern is observed in data[110], and no focusing parameter shift unambiguously causes the small data-MC disagreement seen in the pattern.

\section{A.2.3 MINERvA detector face "daisy" bin ratios}

The most powerful method to search for a focusing cause of the data-MC discrepancy turned out to be shifted flux to nominal flux ratios calculated within "daisy" bins of the hexagonal face of the MINERvA detector (shown in Figure A.10).

The low- $\nu$ data and MC of Figure A.7 divided into their respective daisy bins is shown in Figure A.11. Note that the shape of the discrepancy is similar in each daisy region. Compared to fluxes simulated with shifted parameters, parameters which shift a focusing component perpendicular to the beam direction are always observed to shift the flux differently across the daisy regions. The converse is also true: parameters which do not shift a focusing component perpendicular to the direction of the beam (i.e. parameters whose shift is axially symmetric) are always observed to shift the flux equally in all daisy regions. Compare, for example in Figure A.12, the ratios in daisy regions for a vertical movement in the horn 1 position to an adjustment in the size of the proton beam spot size.

The data-MC discrepancy — if it is caused by a focusing mismodeling — is of the latter type, i.e. it would be caused by an axially symmetric shift in a focusing parameter.

Among such axially symmetric shifts, the z-position of the target (Figure A.13) most closely resembles the discrepancy. But to obtain magnitudes comparable to the data-MC disagreement, a $4 \mathrm{~cm}$ pull (corresponding to $40 \sigma$ from the values measured tolerance) is 
Mean Vertex Position vs Energy -- nominal

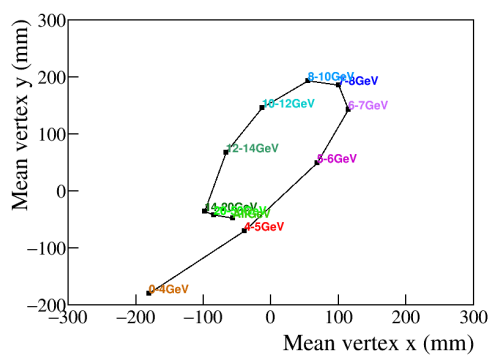

(a) Nominal flux simulation
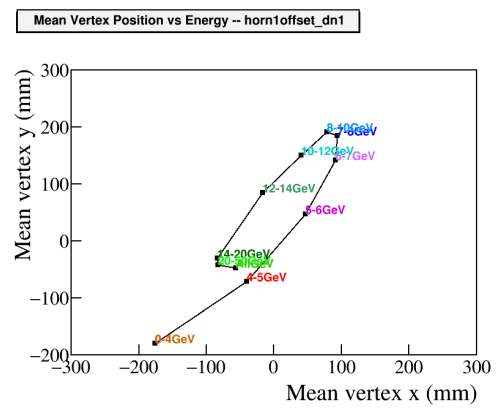

(c) Horn 1 x-position -1 mm

\section{Mean Vertex Position vs Energy - beamXposition_dn1}

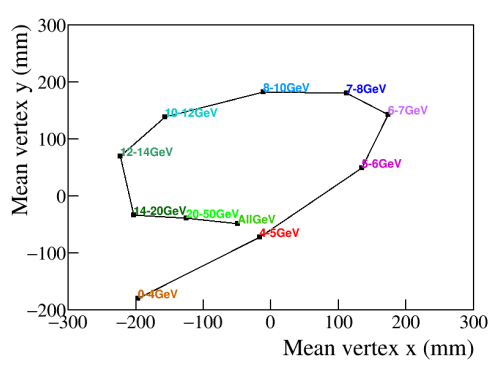

(e) Proton beam x-position $-1 \mathrm{~mm}$
Mean Vertex Position vs Energy - beamsigmaXY_up1

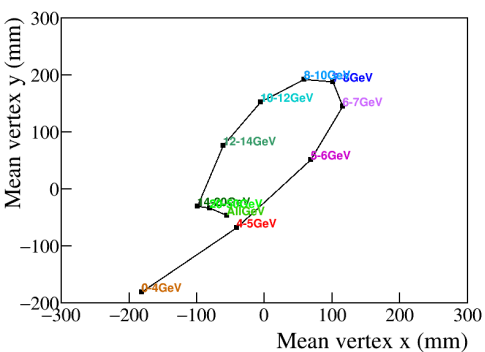

(b) Proton beam spot size $+0.3 \mathrm{~mm}$

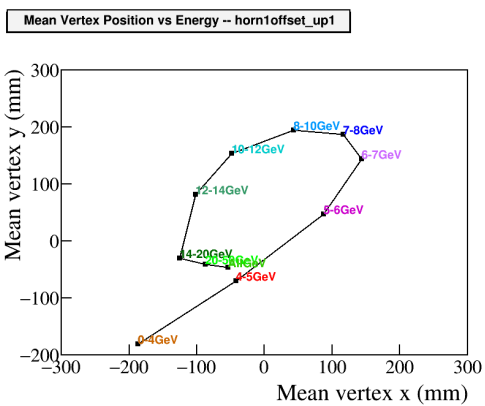

(d) Horn $1 \mathrm{x}$-position $+1 \mathrm{~mm}$

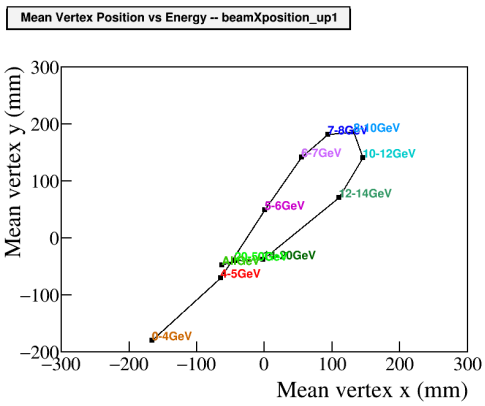

(f) Proton beam x-position $+1 \mathrm{~mm}$

Figure A.9: Mean neutrino x-y interaction position for the flux generated with shifted parameters. 


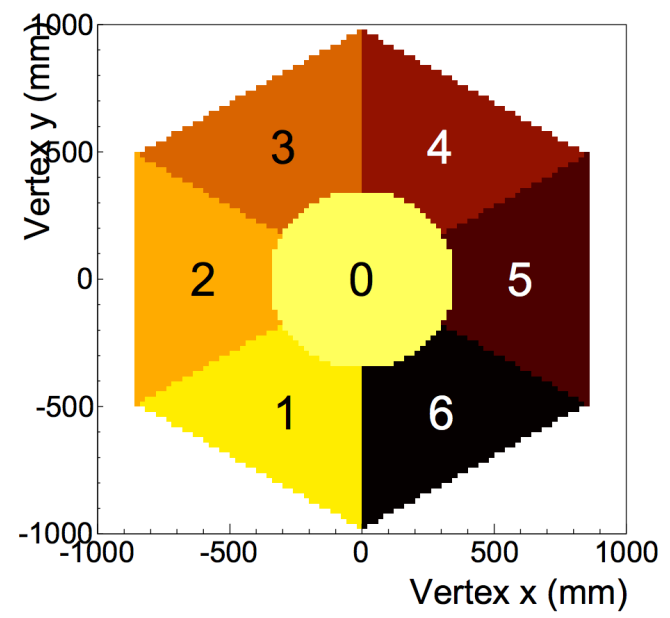

Figure A.10: Daisy binning scheme on the front face of MINERvA.

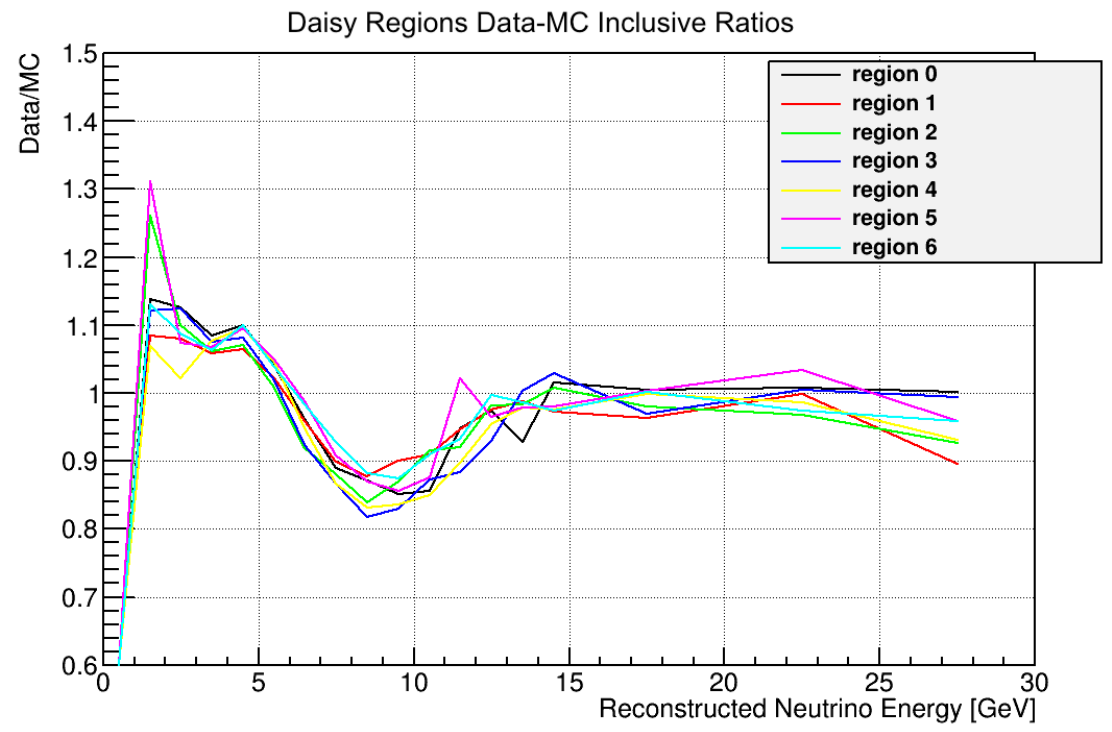

Figure A.11: Low- $\nu$ inclusive event rate Data-MC ratio. The discrepancy shape is similar in each daisy region.

required. A survey of the target position nonetheless confirmed the position to within the stated $1 \mathrm{~mm}$ error. 


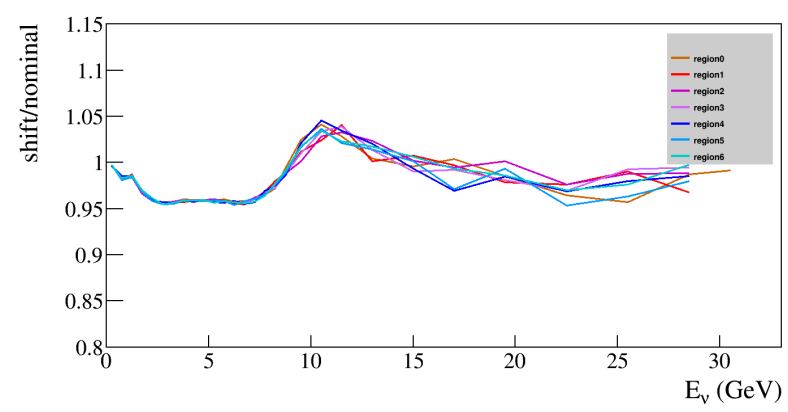

(a) Flux ratios in all daisy regions comparing an increase in proton beam spot size to the nominal prediction. This is an example of a shift that is not perpendicular to the beamline direction, and thus affects each daisy region equally.

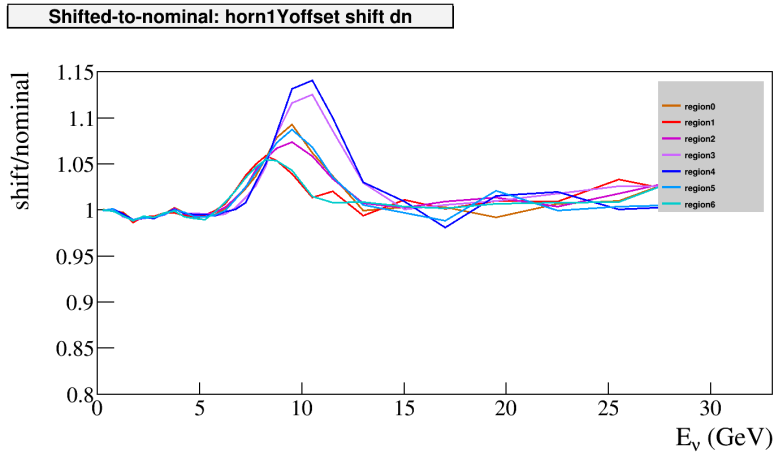

(b) Flux ratios in all daisy regions comparing a shift in vertical horn 1 position to the nominal prediction. This is an example of a shift that is perpendicular to the beamline direction, and thus affects each daisy region differently.

Figure A.12: Daisy bin flux ratios for a proton beam spot size and vertical horn 1 position shift.

The primary conclusion of these studies is that no currently-tunable mismodeled focusing parameter is the cause of the observed discrepancy. Further, the cause has an axially symmetric effect, and it resembles a large shift in the target longitudinal position. In the process of performing these studies, many focusing parameters, those in table 7.4 and many 


\section{Shifted-to-nominal: targetZposition shift up}

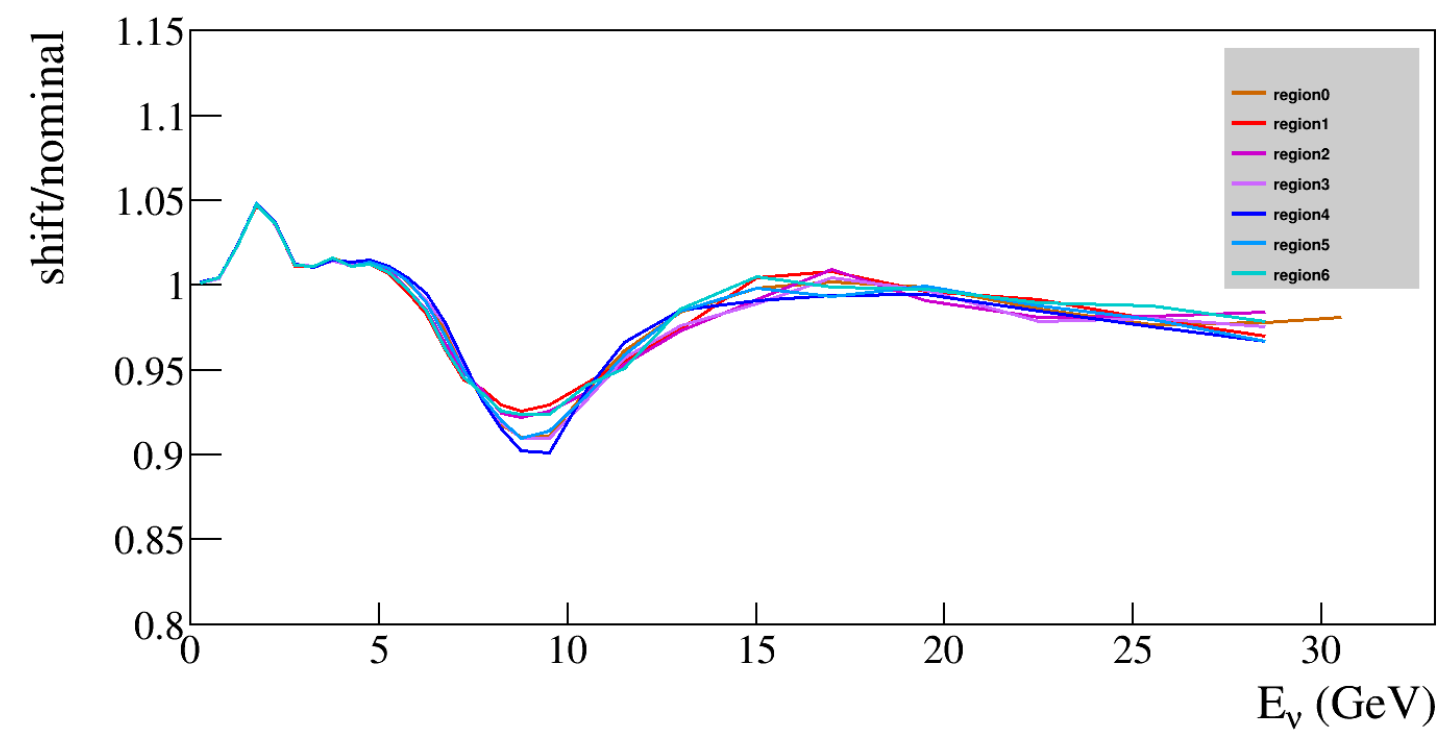

Figure A.13: Daisy region flux ratios of target z-position $4 \mathrm{~cm}$ shift to nominal simulation. $4 \mathrm{~cm}$ corresponds to $40 \sigma$ away from the target z-position measured tolerance.

more, were stretched within and beyond their tolerances and plotted in daisy bins with their ratio to the nominal prediction $[85,87]$. From these, insights about shielding, asymmetries in beam design, and unintuitive effects can be drawn.

\section{A.2.4 Resolution to the discrepancy}

Ultimately, evidence arose, from the work of other members of the collaboration, suggesting that the source of the discrepancy might not be a problem with the flux model (nor, related to the knowledge of the standard candle cross section) but was instead a problem with event reconstruction. Specifically, a $1.75 \sigma$ MINOS muon energy mis-calibration was shown to closely resemble the discrepancy, as shown in Figure A.14.

No obvious explanation for a muon energy scale miscalibration was found, but a problem with the energy scale measurement and uncertainty estimation of the measurement also 
could not be ruled out.

In a joint fit of the focusing parameters and muon energy scale, the focusing parameters were found to be pulled within one standard deviation of their central values while the muon energy scale was pulled by 1.6 or 1.75 standard deviations, depending on whether prior constraints were used. The MINERvA collaboration decided to use the original flux prediction while adjusting the muon energy calculation in data according to the fit.

The joint fit, furthermore ascribed very small uncertainties to the new value of the muon energy scale, viz. the fit suggested that the energy scale uncertainty could be reduced from its nominal value of $2 \%$, originally determined from MINOS calibration, down to $1 \%$. The complete implications of this conclusion are still being investigated, but the aforementioned modifications were used for this thesis measurement.

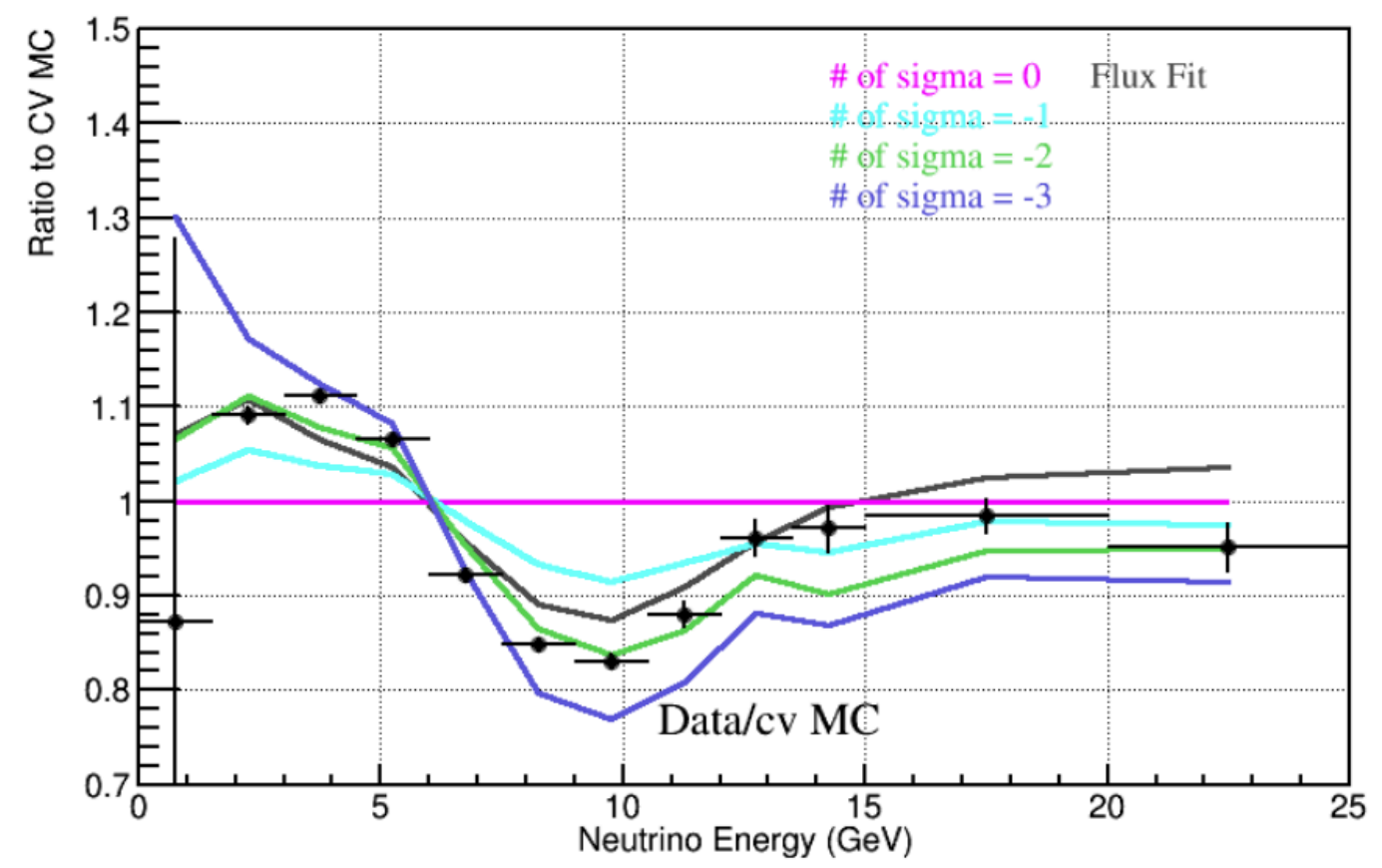

Figure A.14: $E_{\nu}$ ratio for shifts in the reconstructed muon energy compared to nominal prediction, together with data compared to nominal prediction and a fit to the focusing parameters (black line), for the low- $\nu$ data sample. Figure from [50]. 


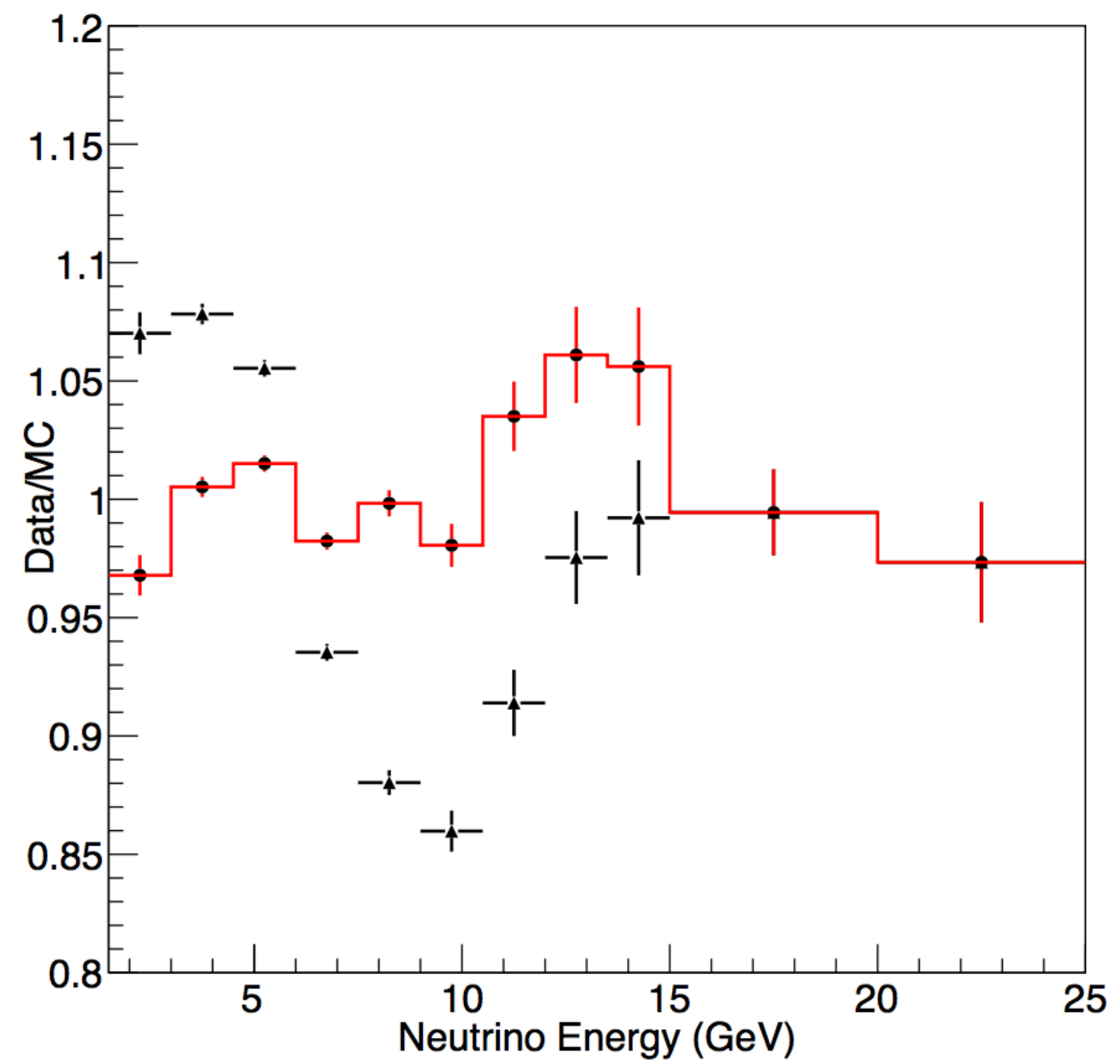

Figure A.15: Ratios of low- $\nu$ sample data-to-MC and nominal-to- $1.75 \sigma$ shifted muon energy scale MC. 


\section{APPENDIX B. CROSS SECTION SYSTEMATIC UNCERTAINTY BREAKDOWNS}

\section{B.1 DETECTOR UNCERTAINTIES}




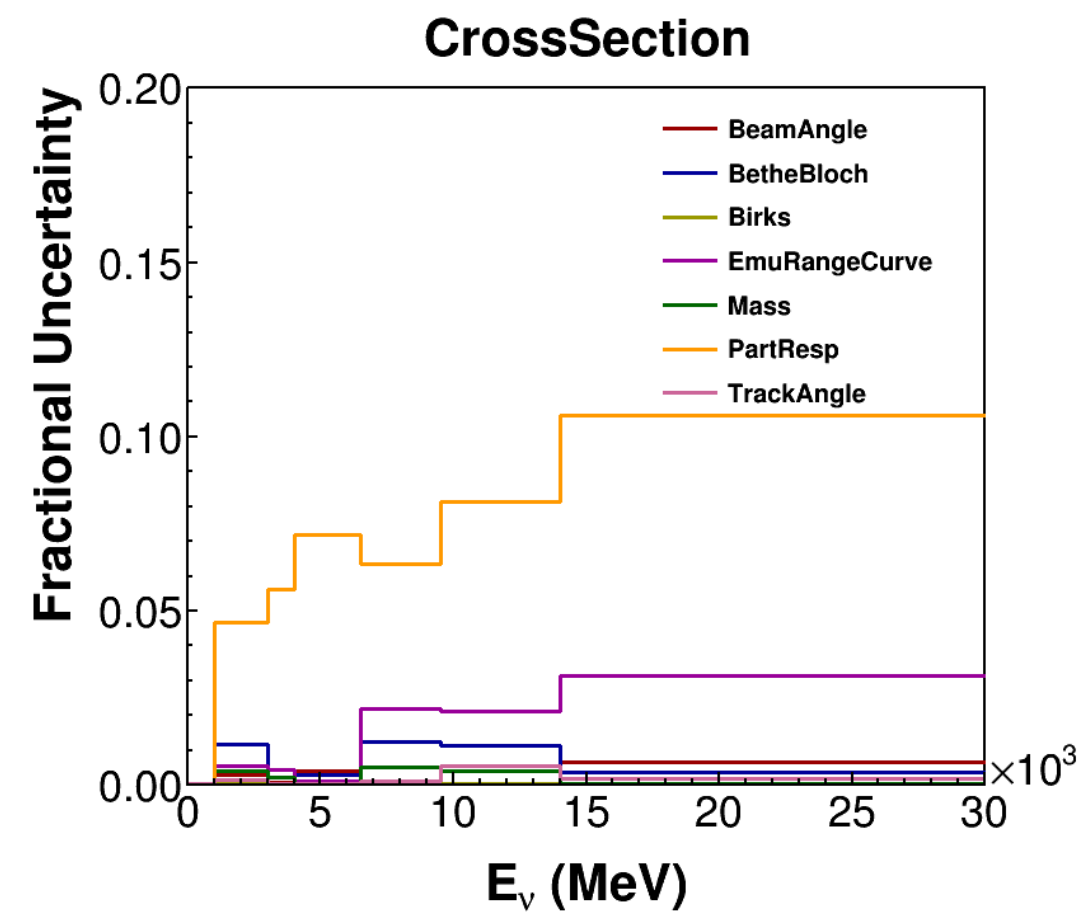

(a) $E_{\nu}$ detector cross section uncertainties

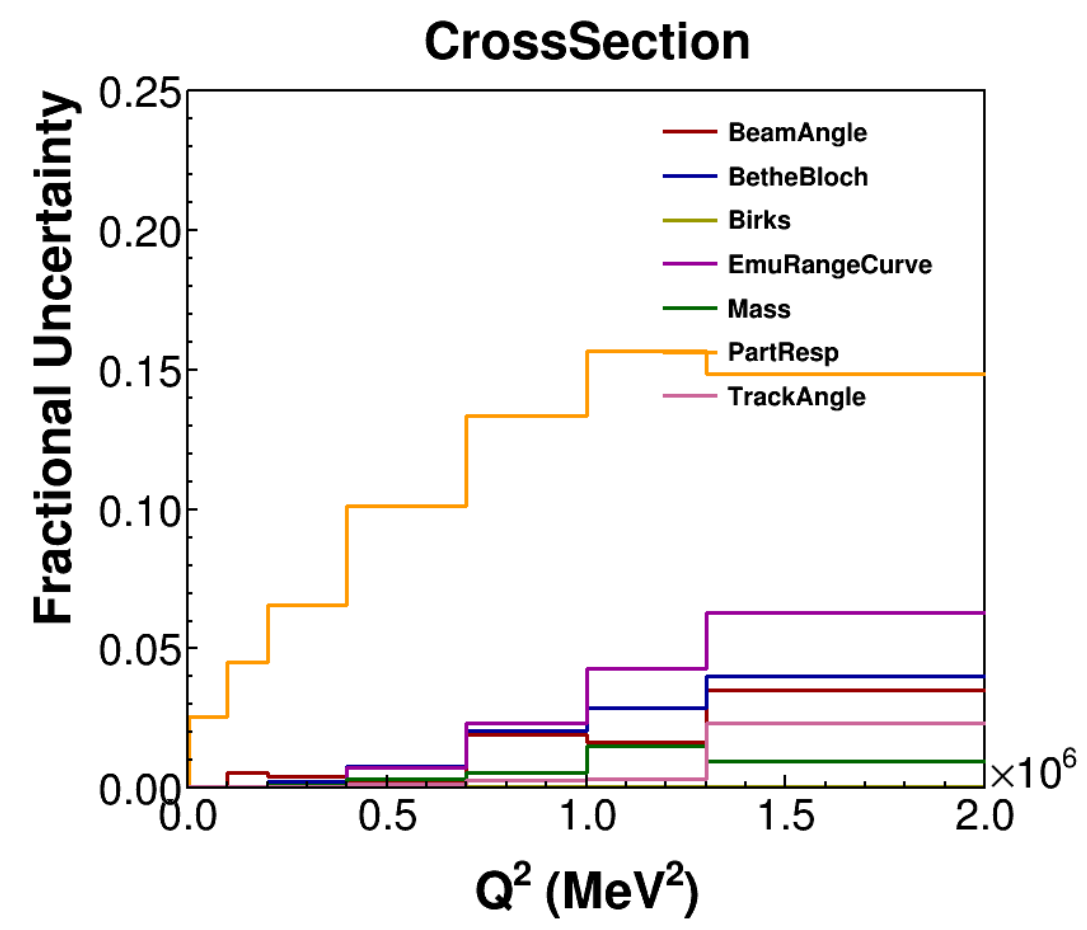

(b) $Q^{2}$ detector cross section uncertainties 


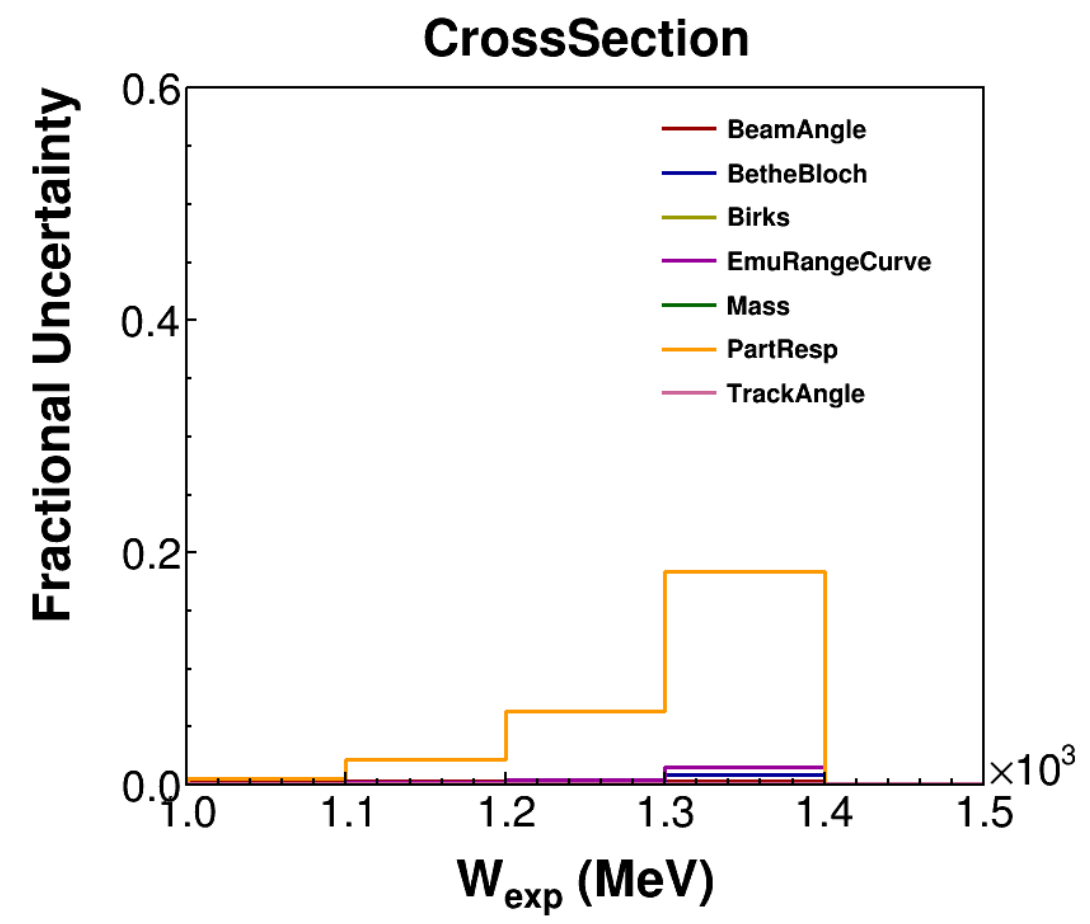

(c) $W_{\text {exp }}$ detector cross section uncertainties

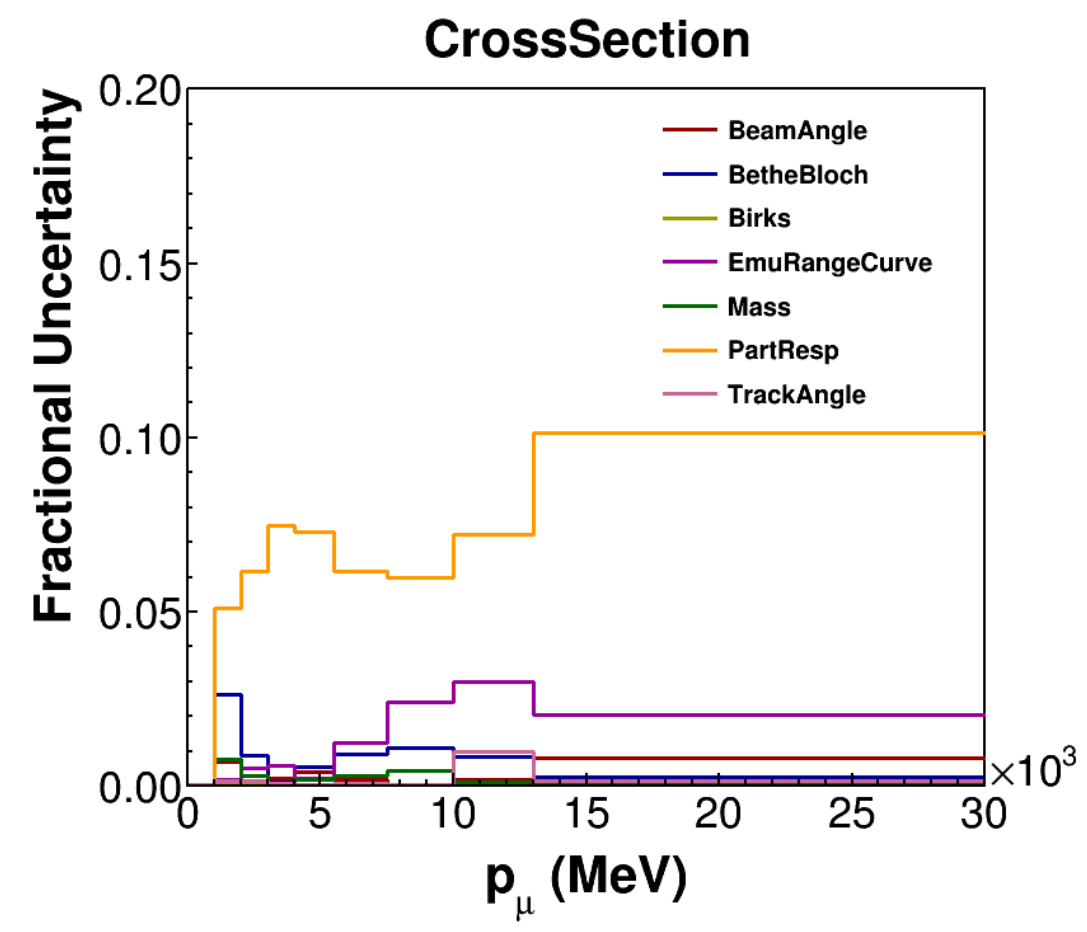

(d) $P_{\mu}$ detector cross section uncertainties 


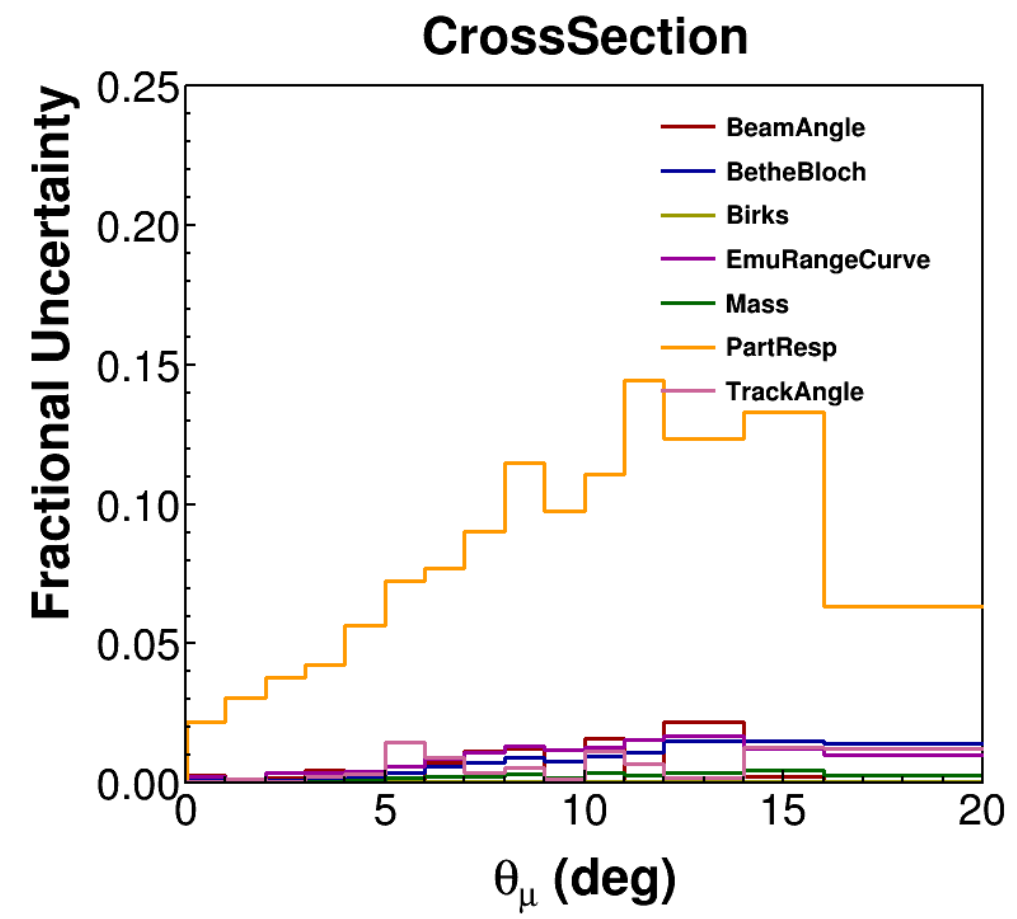

(e) $\theta_{\mu}$ detector cross section uncertainties

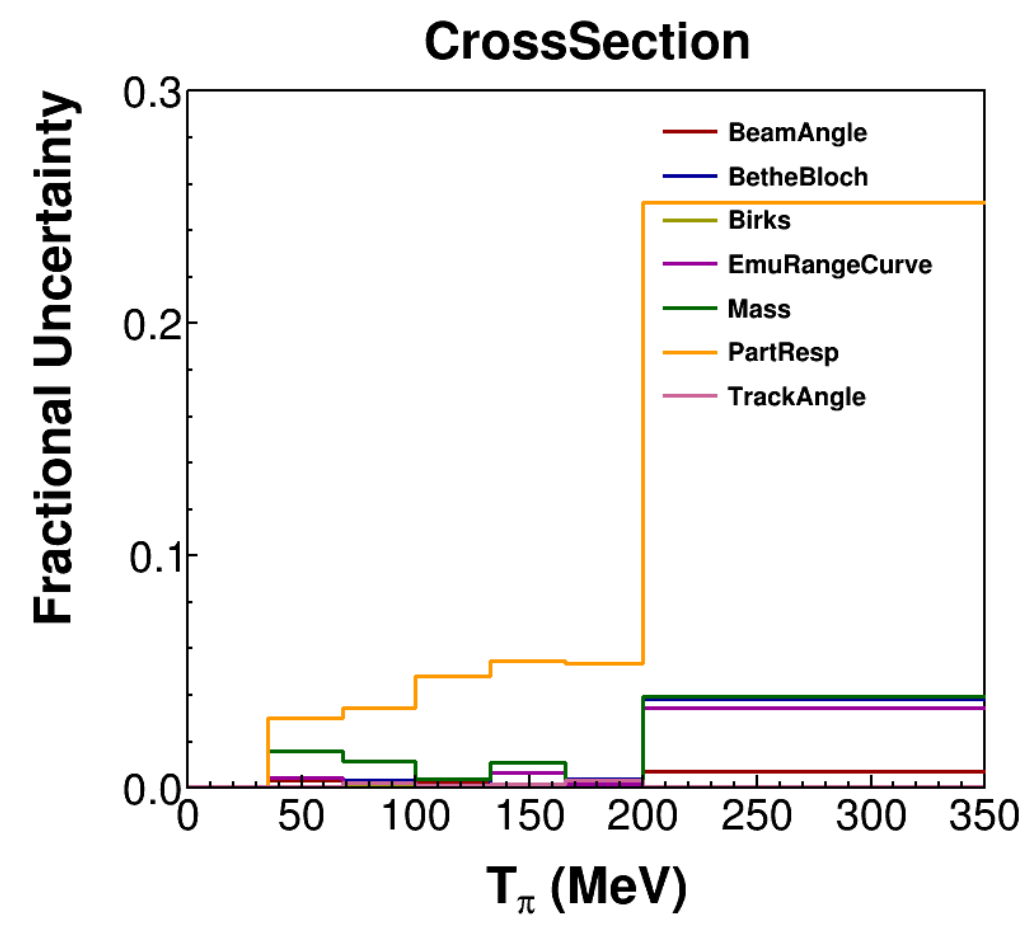

(f) $T_{\pi}$ detector cross section uncertainties 


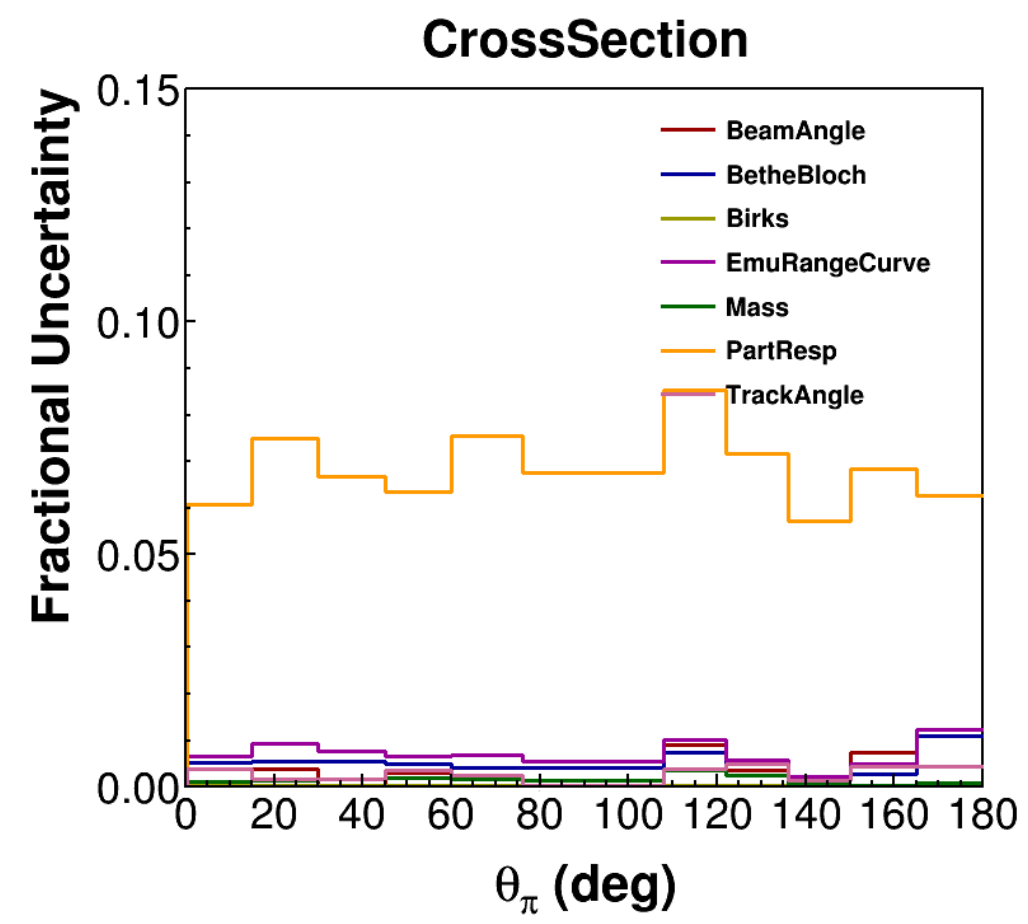

(g) $\theta_{\pi}$ detector cross section uncertainties

Figure B.1: Cross section uncertainties due to detector effects. Only the largest contributions are shown. 


\section{B.2 GENIE INTERACTION MODEL UNCERTAINTIES}

Legend abbreviations:

- MaCCQE CCQE axial mass

- MaRES Resonant axial mass

- MvRES Resonant vector mass

- NormCCRES CC resonant normalization

- Rvp2pi Normalization of 2- $\pi$ final states $(\nu+p)$

- Rvn2pi Normalization of 2- $\pi$ final states $(\nu+n)$ 


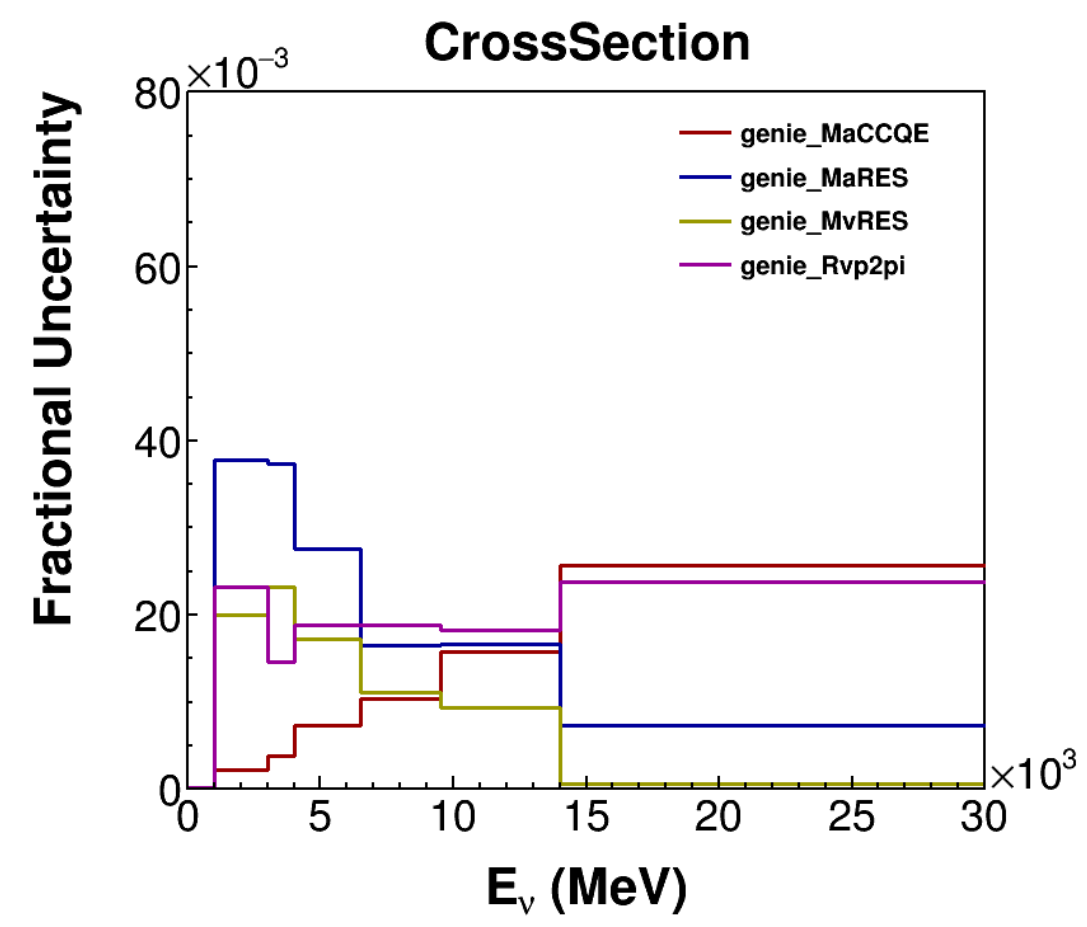

(a) $E_{\nu}$ GENIE interaction model cross section uncertainties

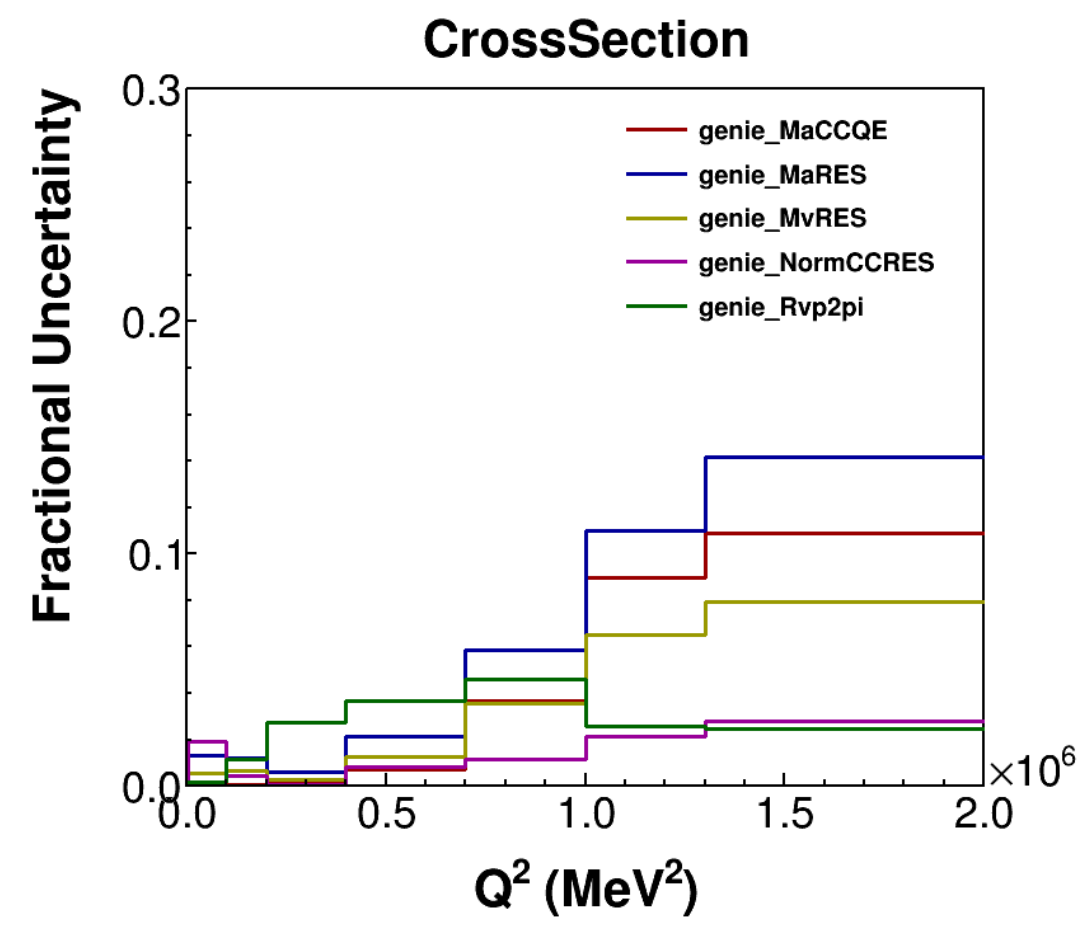

(b) $Q^{2}$ GENIE interaction model cross section uncertainties 


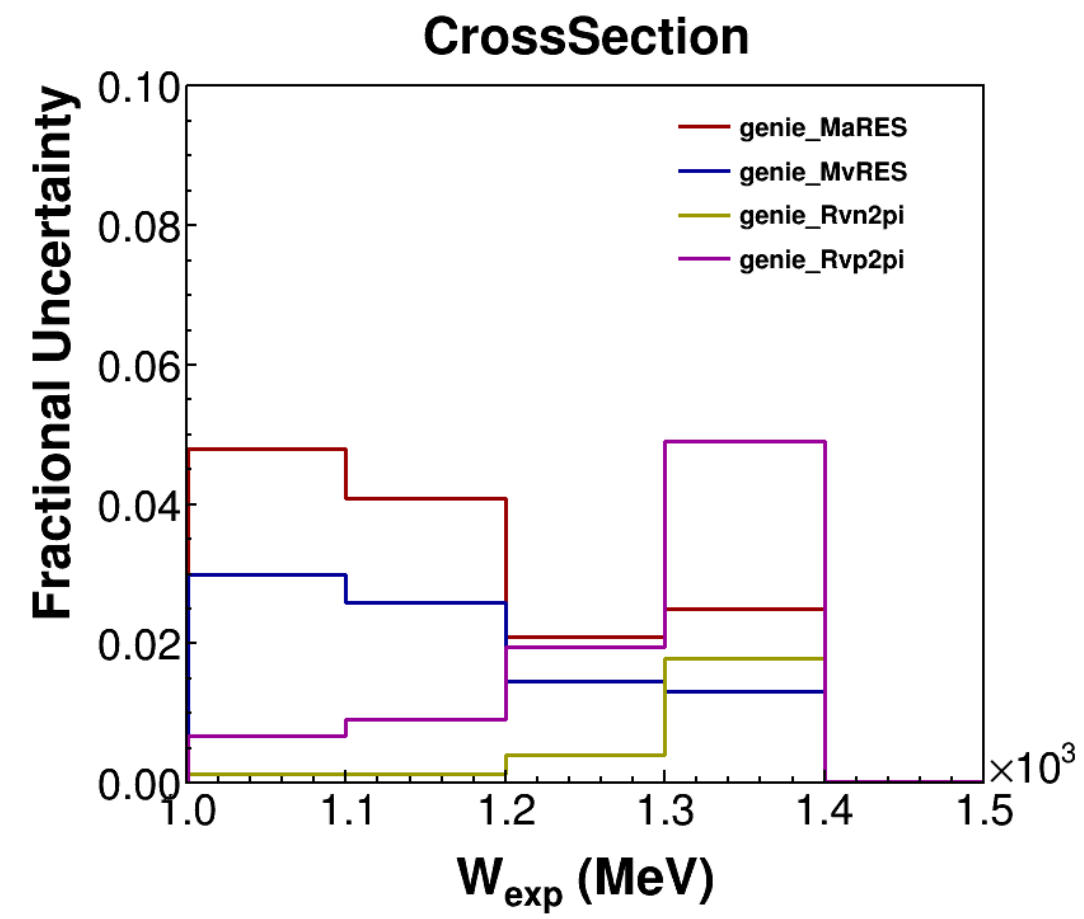

(c) $W_{\text {exp }}$ GENIE interaction model cross section uncertainties

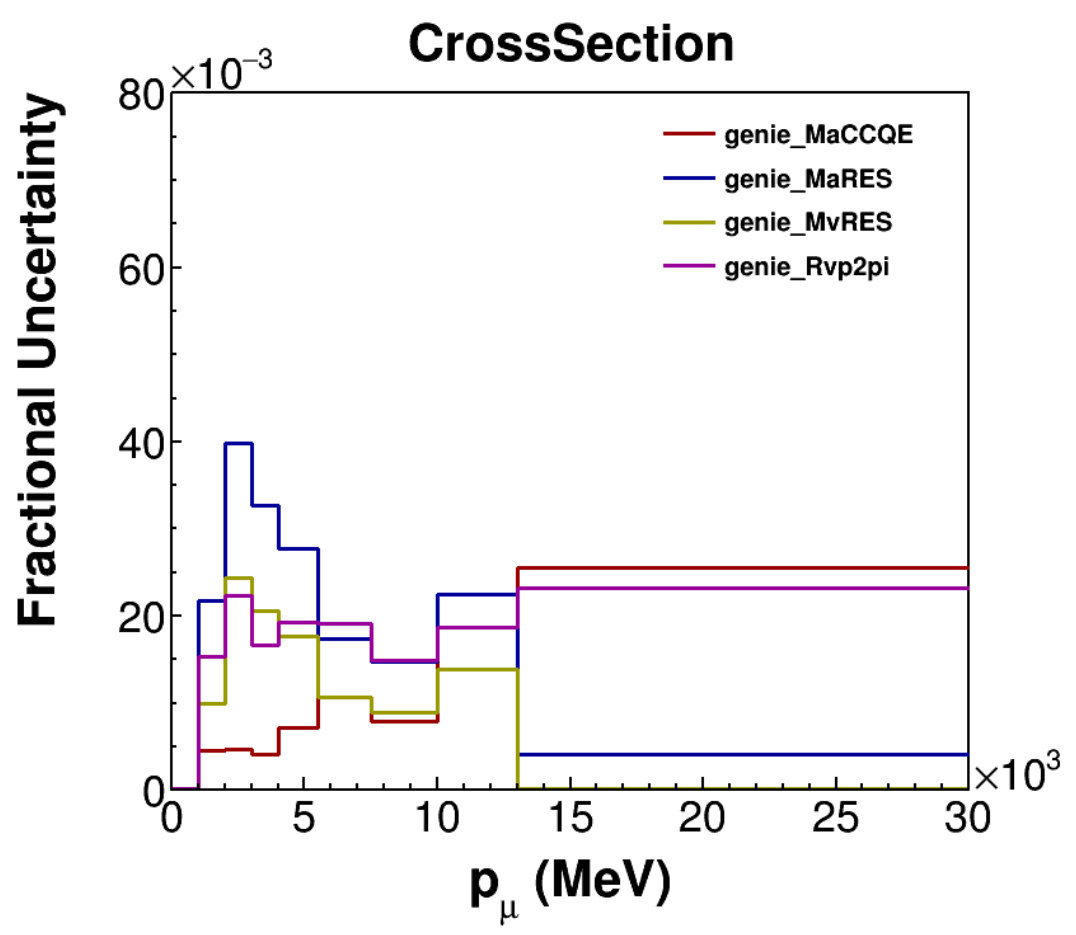

(d) $P_{\mu}$ GENIE interaction model cross section uncertainties 


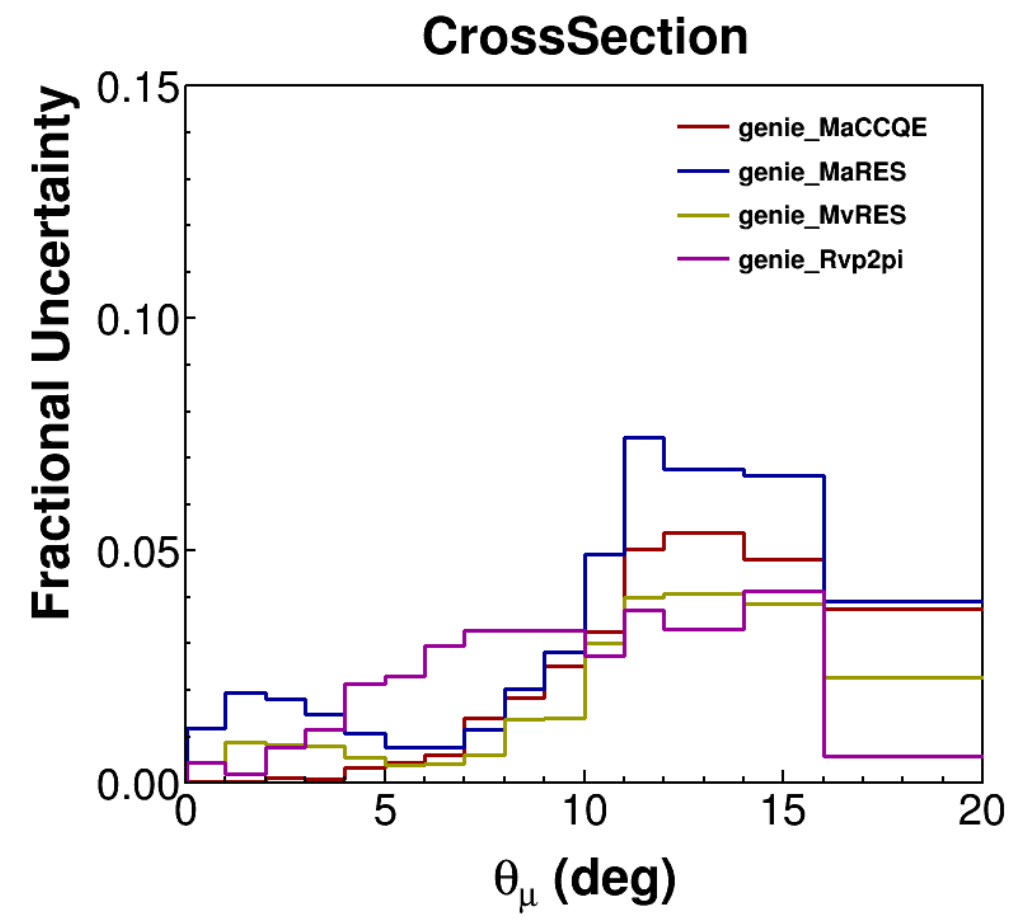

(e) $\theta_{\mu}$ GENIE interaction model cross section uncertainties

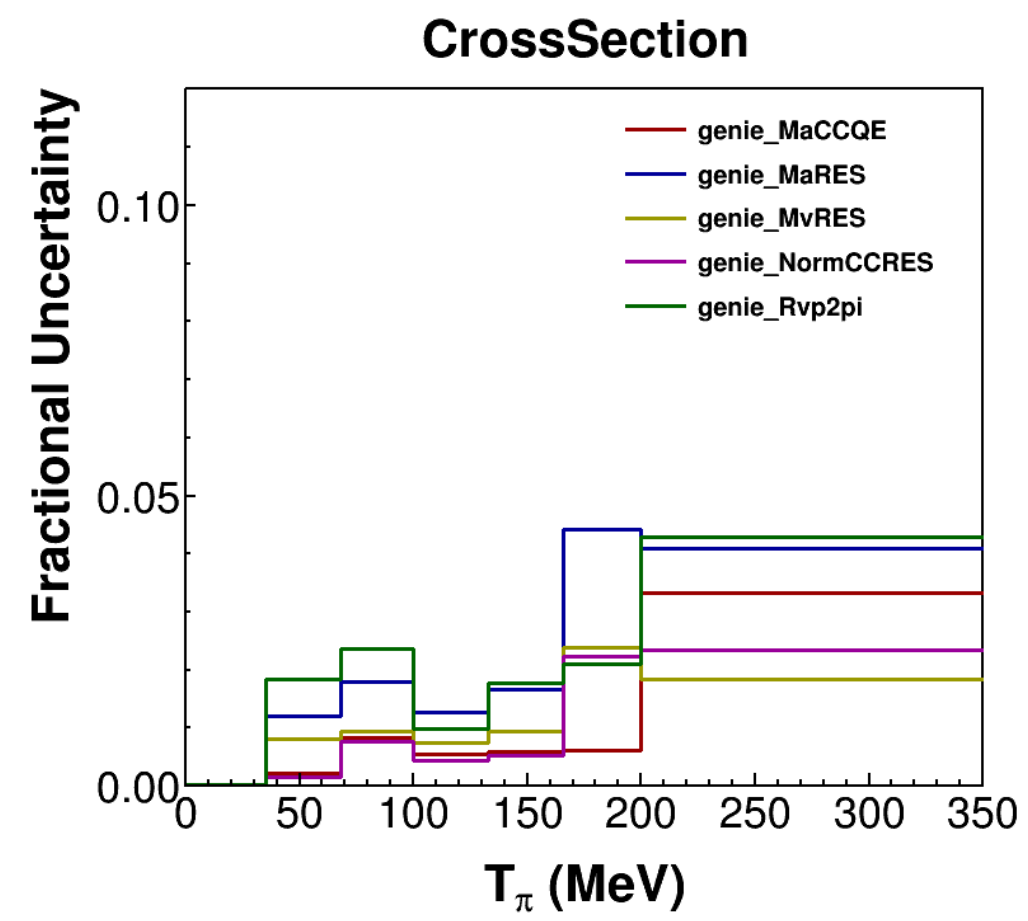

(f) $T_{\pi}$ GENIE interaction model cross section uncertainties 


\section{CrossSection}

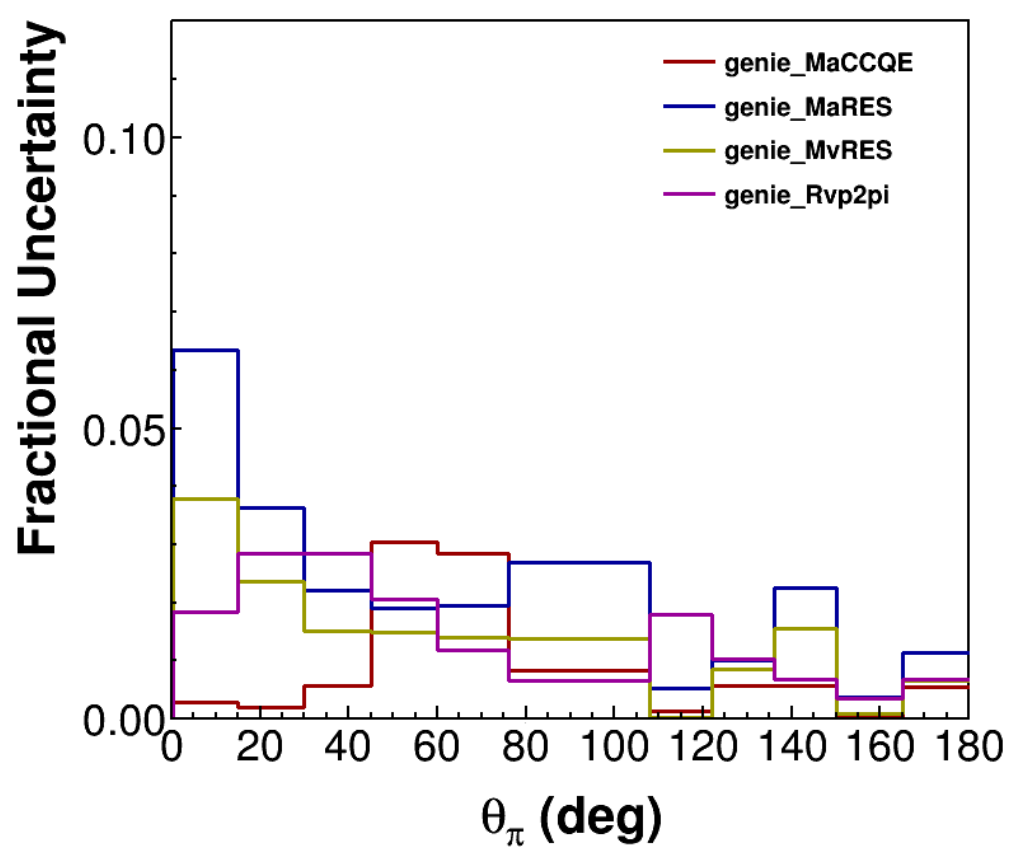

(g) $\theta_{\pi}$ GENIE interaction model cross section uncertainties

Figure B.2: Cross section uncertainties due to GENIE interaction model effects. Only the largest contributions are shown. 


\section{B.3 GENIE FSI UNCERTAINTIES}

Legend abbreviations:

- FrAbs_X Pion/Nucleon absorption probability

- FrElas_X Pion/Nucleon elastic interaction probability

- FrInel_X Pion/Nucleon inelastic interaction probability

- FrPiProd_X Pion/Nucleon pion production probability

- MFP_X Pion/Nucleon mean free path

- Theta_Delta2NPi Pion angular distribution in $\Delta$ decay 


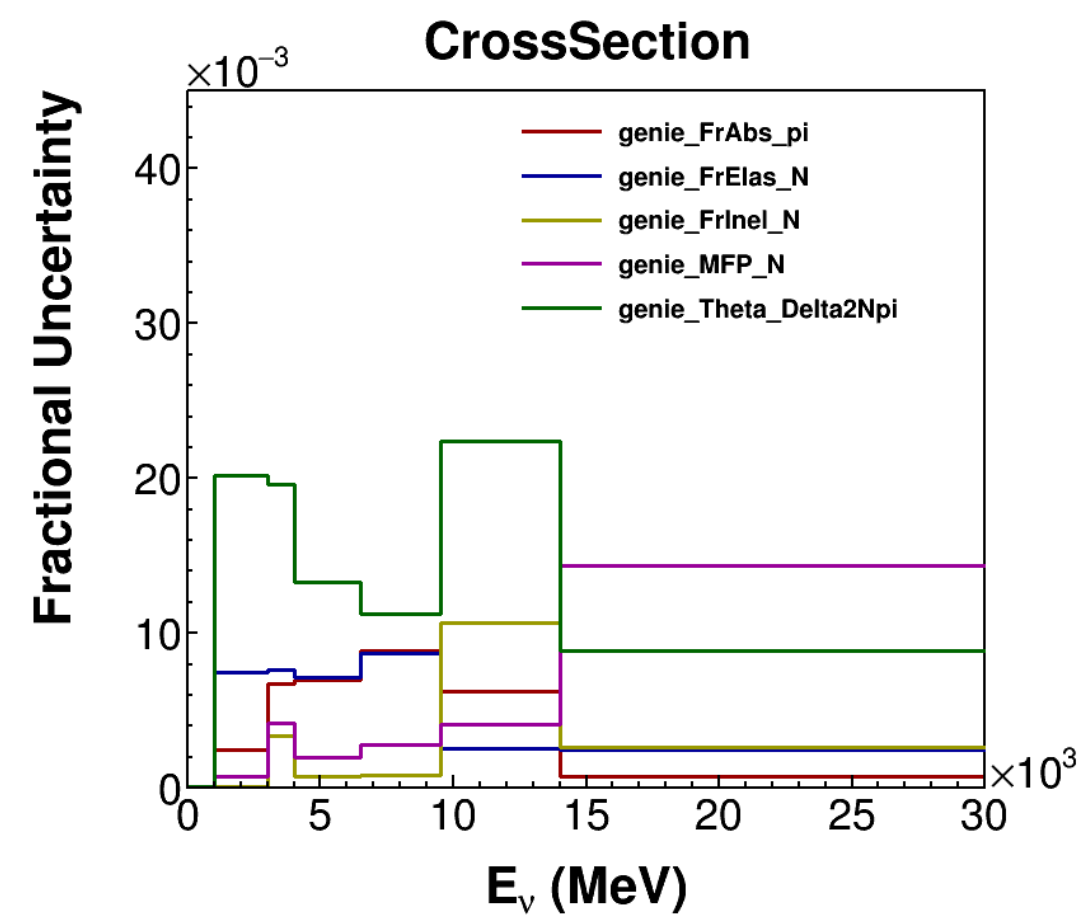

(a) $E_{\nu}$ GENIE FSI cross section uncertainties

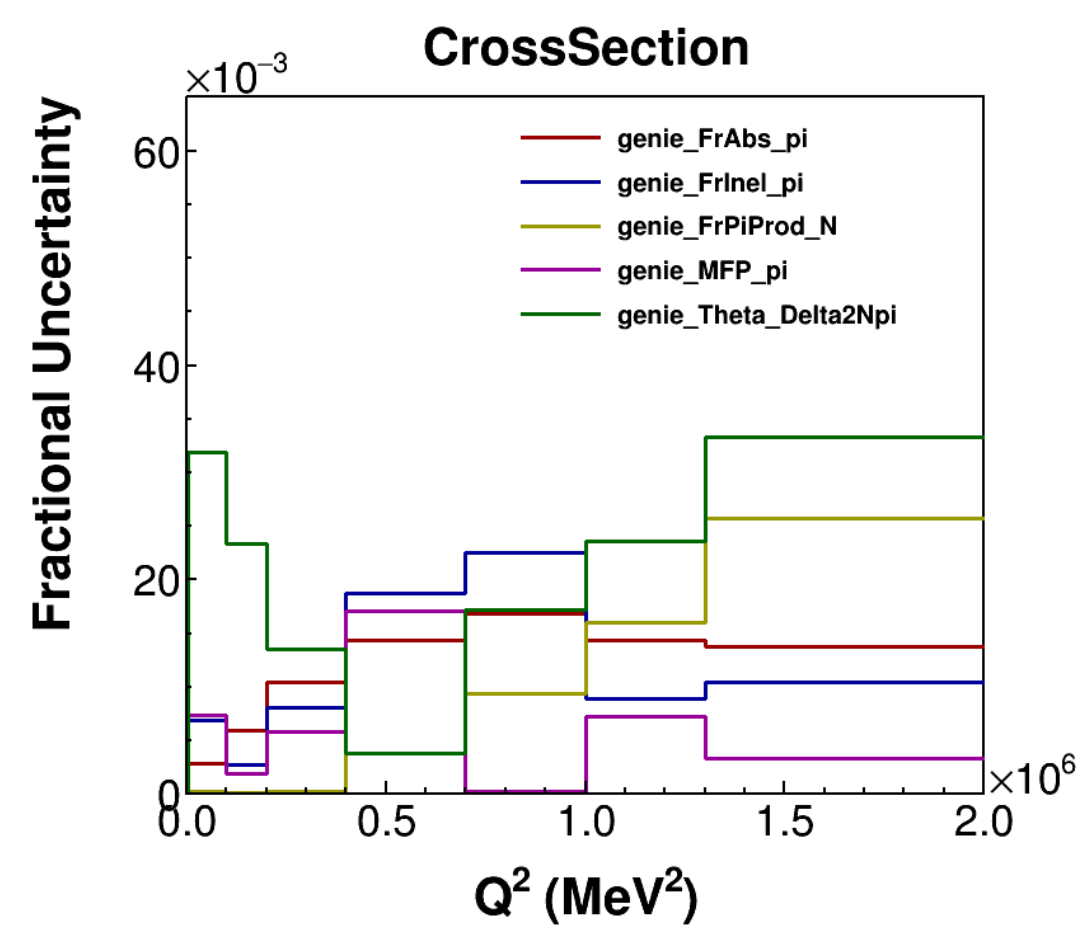

(b) $Q^{2}$ GENIE FSI cross section uncertainties 


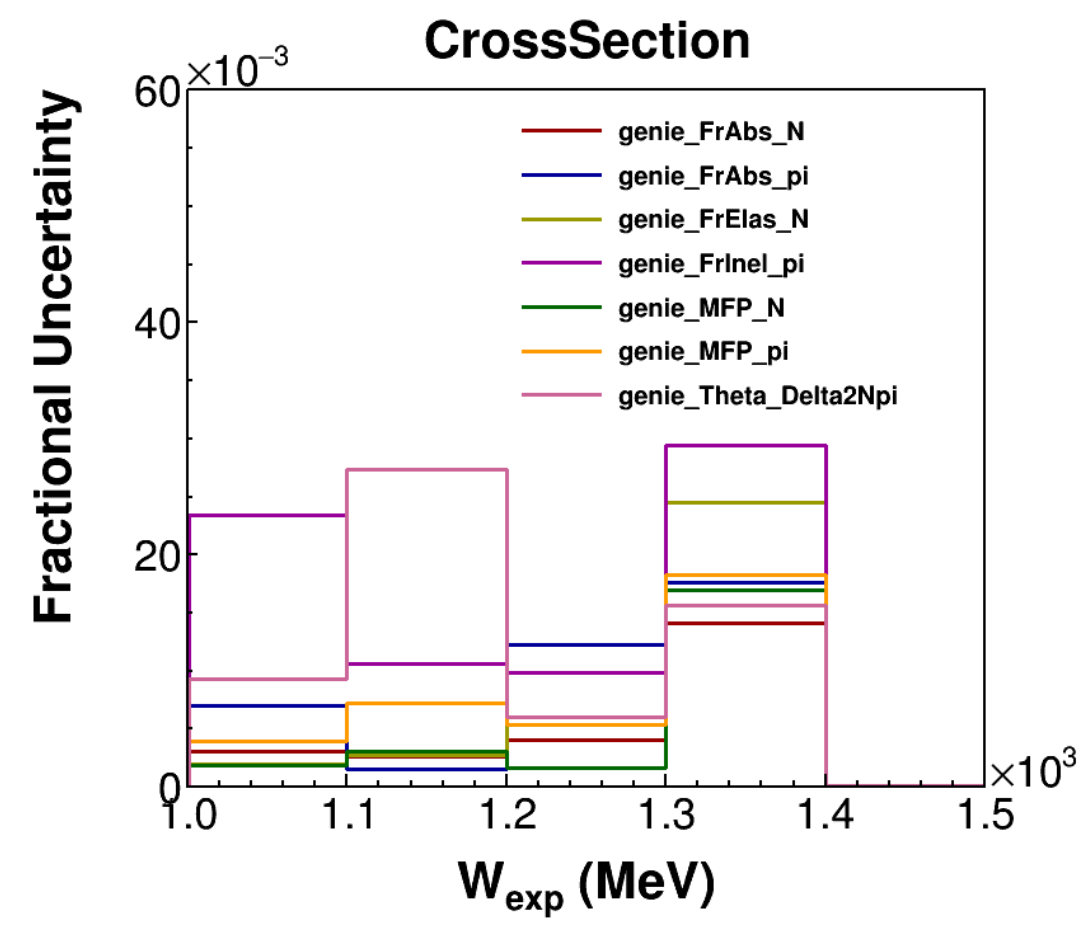

(c) $W_{\text {exp }}$ GENIE FSI cross section uncertainties

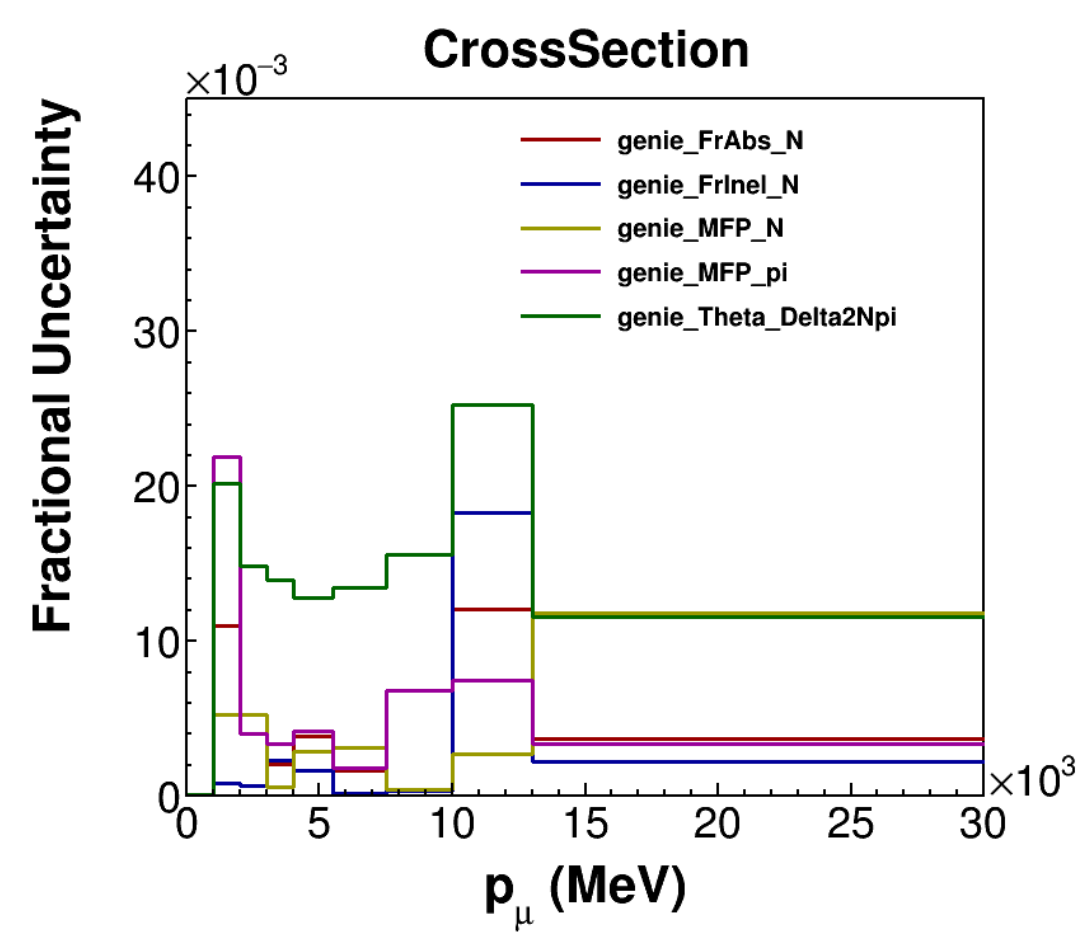

(d) $P_{\mu}$ GENIE FSI cross section uncertainties 


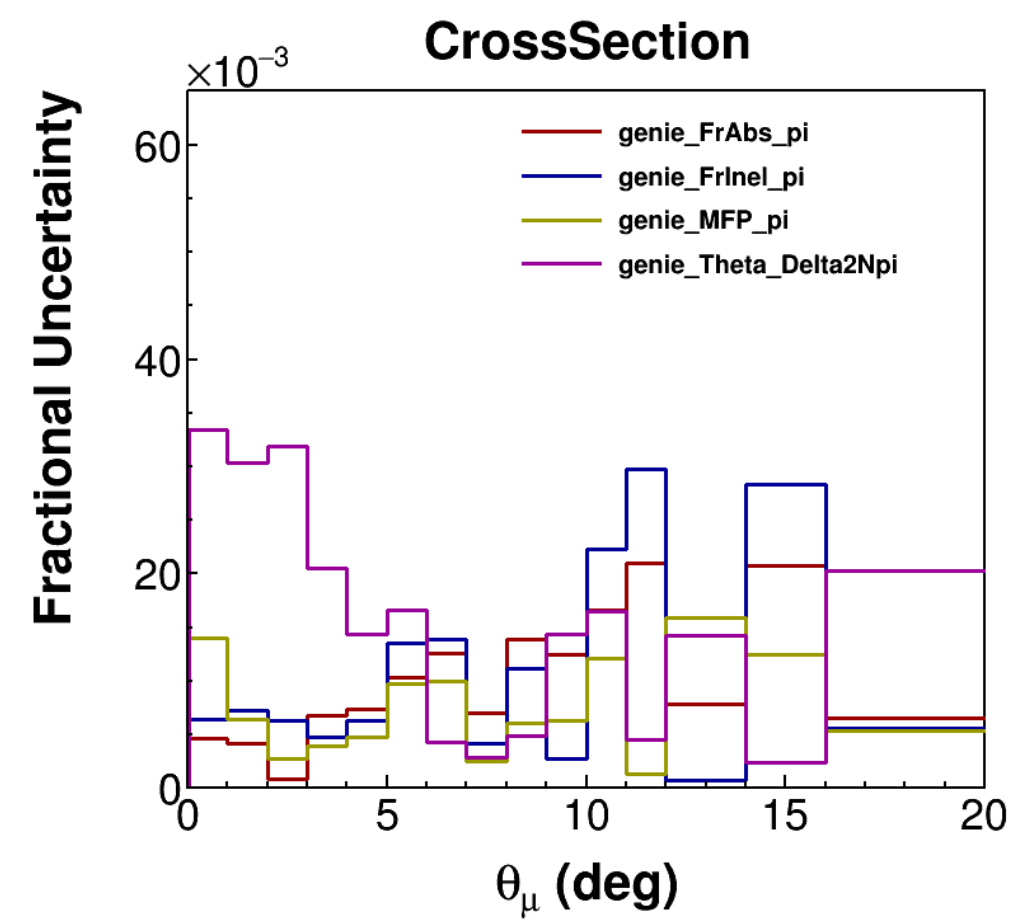

(e) $\theta_{\mu}$ GENIE FSI cross section uncertainties

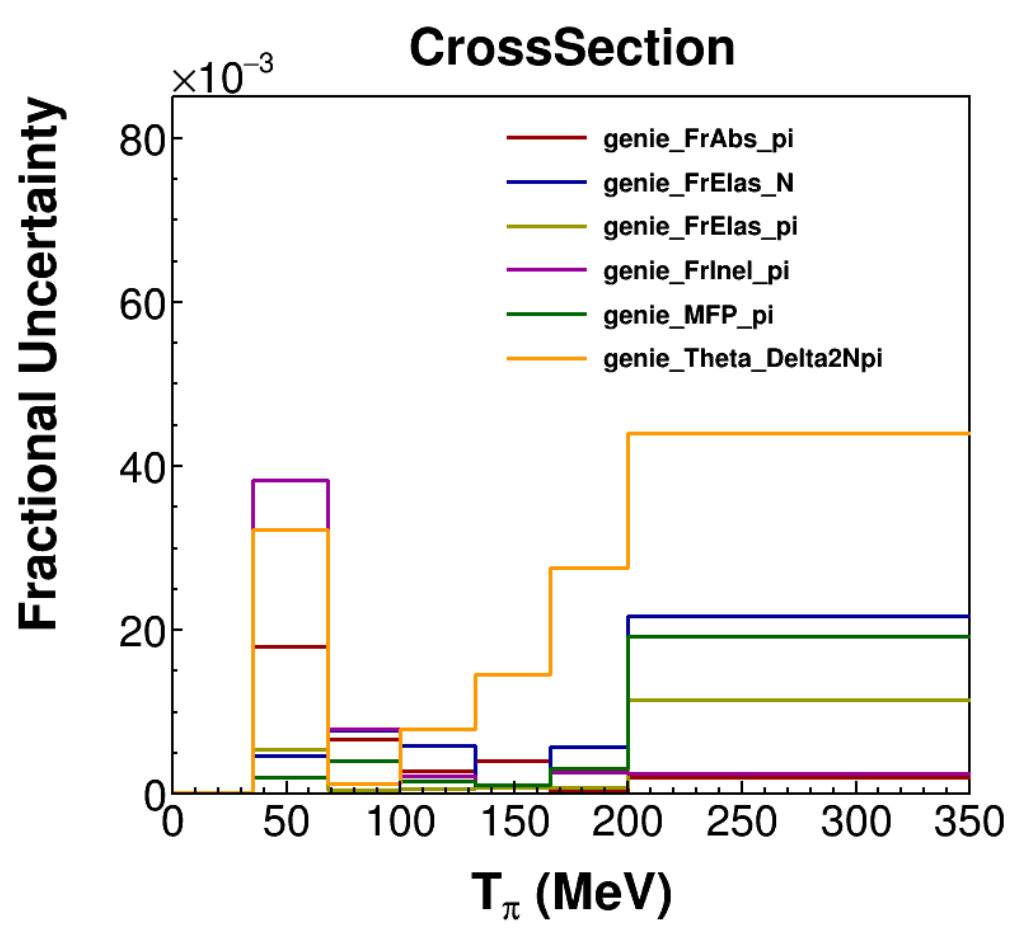

(f) $T_{\pi}$ GENIE FSI cross section uncertainties 


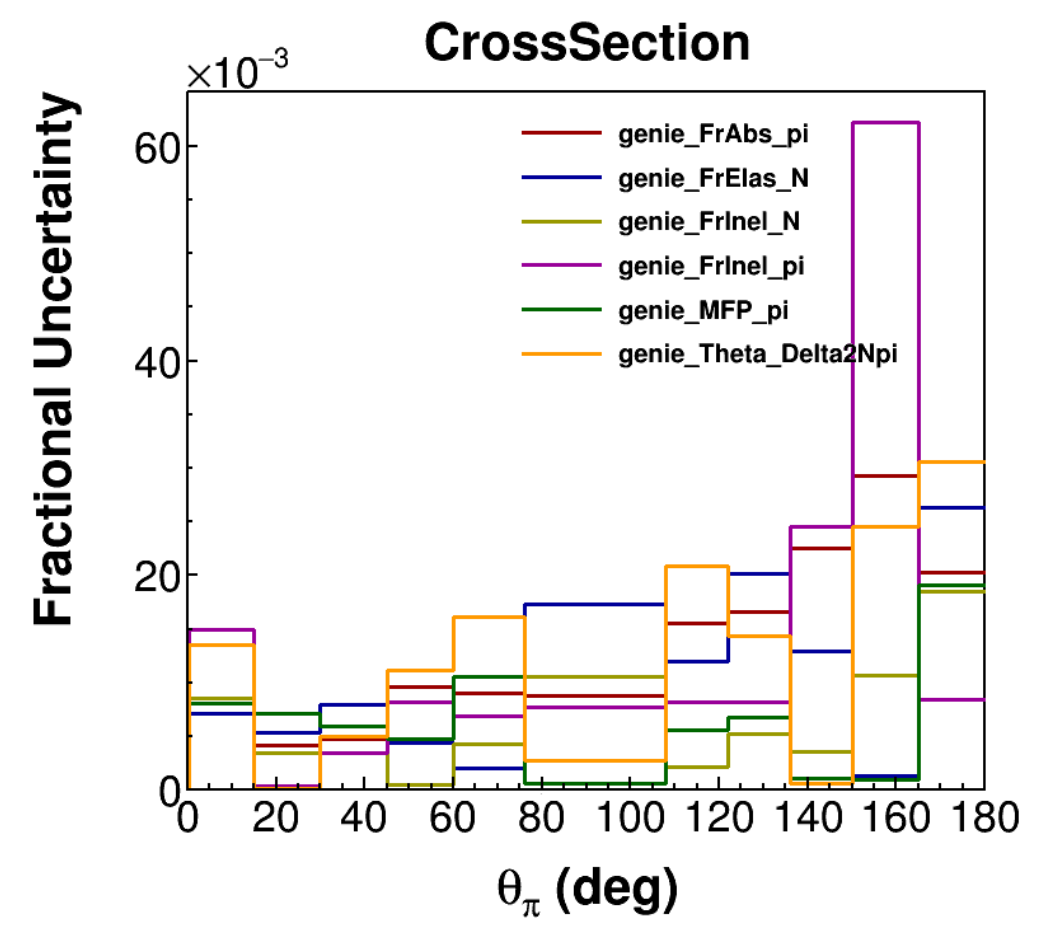

(g) $\theta_{\pi}$ GENIE FSI cross section uncertainties

Figure B.3: Cross section uncertainties due to GENIE FSI effects. Only the largest contributions are shown. 


\section{APPENDIX C. MINERVA MANY-UNIVERSE SYSTEMATICS SOFTWARE DESIGN}

This appendix discusses the design and implementation of the many-universe systematic uncertainty method in the MINERvA software framework. The framework is a software template and prescription for looping over physics events, making selection cuts, and filling event variable histograms - all in many systematic universes from which bin-by-bin uncertainties can be calculated.

More specifically, the framework has two main components: systematic universe classes - which correctly manipulate a physics event in accordance with a systematic shift and a histogram class object called a HistWrapper — which contains a histogram for each systematic universe. There are several pre-defined, MINERvA-standard systematics classes that a user can simply "drag in" to her analysis, but the user can also define her own, analysis-specific systematics classes, inheriting from a base class.

This framework replaces a previous one which was constructed piecemeal by several different authors and which only included many-universes as an afterthought. As a result, the previous method is convoluted and error-prone. Its handling of cuts on laterally-shifted universes is particularly unintuitive. Such complications inherient to many-universes warrant a top-level design.

The new framework in contrast revolves around the many-universe method and proceeds linearly: for each physics event, each systematic universe is considered - whether or not it passes selection cuts - in turn. Additionally, it takes advantage of new technologies for speed increases, parallel grid computing, and python compatibility.

MINERvA is transitioning future analyses to the framework, and it will serve as the the analysis software foundation for measurements in MINERvA's "data preseravation" and 
"open science" era, ocurring past the lifetime of the collaboration itself.

\section{C.1 BASIC SYSTEMATICS FRAMEWORK USE}

Pseudocode snippet C.1 shows the basics of the framework use. First, systematic universe objects are collected in a container. In this container, for example, there may be a central value, unshifted universe object, a universe for an up-shift to the muon energy, and a universe for a down-shift to the muon energy. Next a HistWrapper object is created from the container of universes, as well as from usual histogram inputs (e.g. binning information, labeling, etc.). Then for each physics event, each universe in the container is considered. Within a universe, event variables are extracted or otherwise calculated and subsequently modified according to the prescription defined in the universe. Whether the event passes selection cuts, in what bin the event belongs, or what weight is applied to the event can all be dependent on the shifted values.

Listing C.1: Pseudocode for using the systematics framework. (L1) Get container of systematic universes including a CV universe, (L2) create a histogram from the universes (here, the "..." includes binning, naming, etc.), (L3-L13) loop physics events, (L4-L12) loop universes, (L5) check if event/universe pass selection cuts, (L8-L10) calculate event/universe variable, event/universe weight, and (L11) fill the histogram.

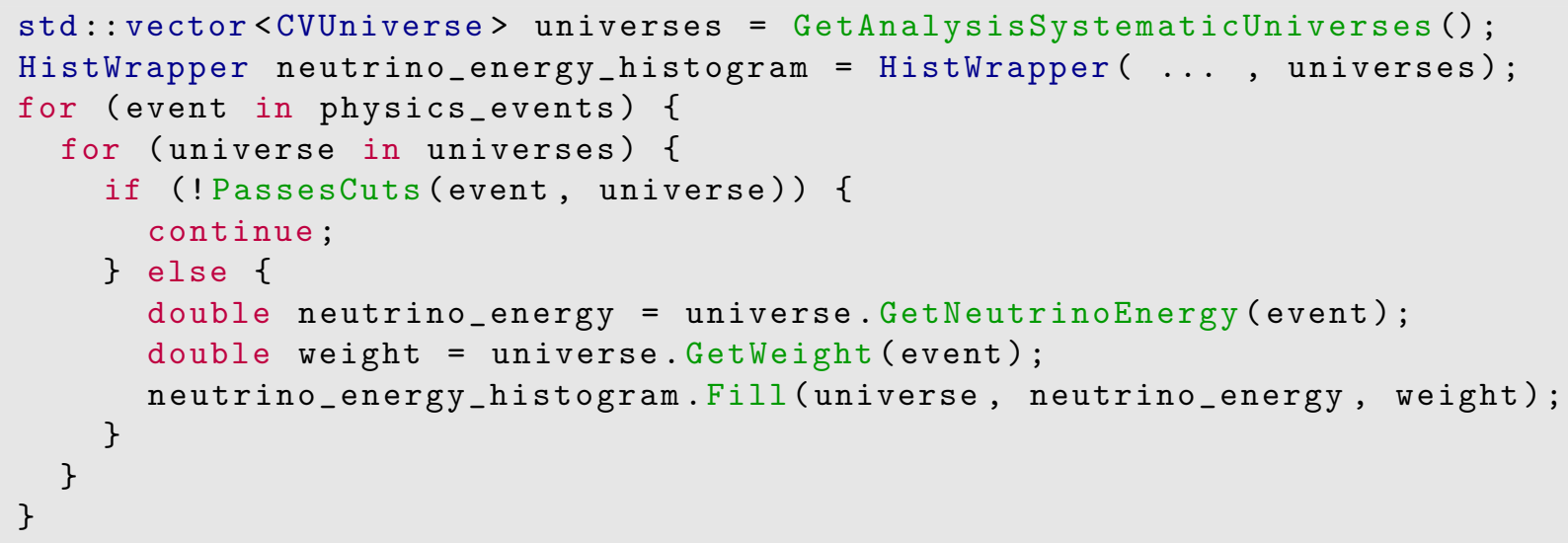

As mentioned above, the framework has two main components: the universe classes and the HistWrapper histogram class. These will be described next. 


\section{C.2 UNIVERSE CLASSES}

Figure C.1 shows the inheritance structure of systematic universe objects. At the top is the DefaultCVUniverse class, an abstract class living in the MINERvA-standard PlotUtils namespace and from which a user's CVUniverse derives. DefaultCVUniverse owns (a container of) all physics events being considered and several generic physics event variable accessors.

The user-defined CVUniverse inherits from DefaultCVUniverse and defines a central value GetWeight function as well as any user-specific event variable calculators and accessors.

Various systematic universes are template classes which expect a template argument class that resembles DefaultCVUniverse (i.e. CVUniverse). These classes override the GetWeight function or other event variable calculators and accessors that were originally defined in CVUniverse. By overriding the functions, the correct universe-version of the function is called within the universe loop, for example in lines L8 and L9 of Listing C.1. There are several, pre-defined standard systematic universes living in the PlotUtils namespace. And the user can write her own, analysis-specific universes.

There are two types of systematic universe shifts: vertical and lateral. Vertical systematic universes override the GetWeight function. These shift the probability that an event happened, and they adjust the contribution made to a histogram bin. GENIE and flux systematics are examples of such systematics. A lateral systematic universe, in contrast overrides event variable accessors or calculators. These systematics, unlike vertical systematics, can change whether an event passes or fails cuts, and they can move events from bin to bin. Detector systematics, such as Birks, particle response, and angle resolution are examples of lateral systematics.

Currently, MINERvA standard systematics are all vertical systematics; they are: Flux, GENIE, MINOS efficiency, and the MnvGENIE weight systematics. Lateral systematics are technically harder to standardize, though several muon energy and angle systematics should soon be added to the framework. 


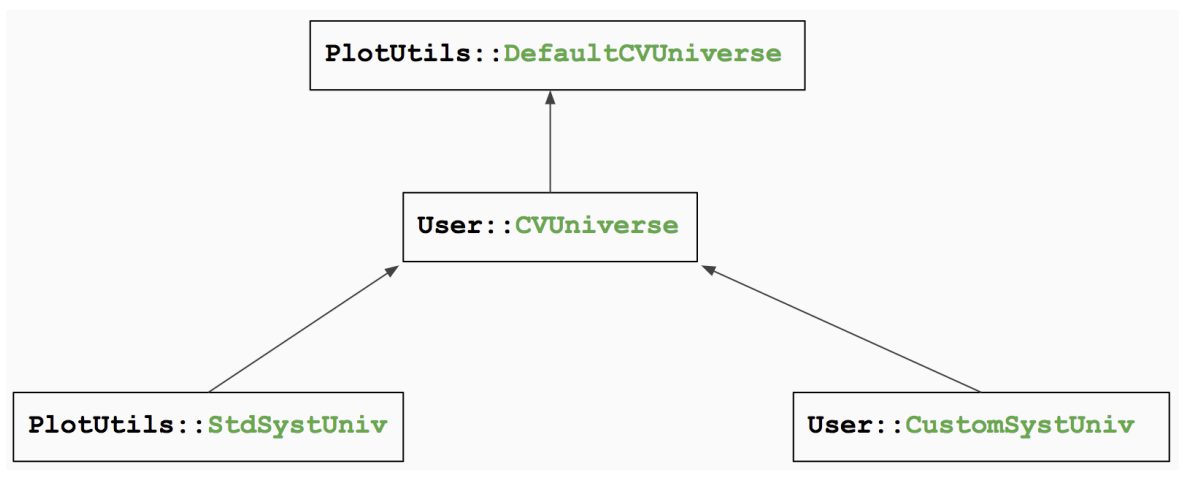

Figure C.1: Systematic universe objects inheritance structure. DefaultCVUniverse is an abstract, standard class living in the PlotUtils namespace. Each user writes her own CVUniverse class which inherits from, expands upon, and overrides the functions of DefaultCVUniverse. Inheriting as template classes from the user's CVUniverse are PlotUtils universe classes, standardized for MINERvA use. Also inheriting from the user's CVUniverse are user-defined, analysis-specific systematic universes.

\section{C.3 THE HISTWRAPPER CLASS}

The HistWrapper holds separate histograms corresponding to each universe. As in line L10 of code snippet C.1, the histogram for the correct universe is filled with the potentially shifted neutrino energy and potentially shifted weight. The HistWrapper thus maps the universe object to the correct histogram.

The HistWrapper is built upon a MnvH1D (MINERvA histogram 1D), which holds a simple 1D histogram corresponding the central value as well as groups of simple 1D histograms, categorized by systematic category. These latter groups are called error bands. More specifically, error bands hold a copy of the central value histogram, as well as the simple 1D histograms for each universe in the band. The muon energy error band, for example, will have an unshifted central value histogram as well as an muon energy up-shift histogram and a muon energy down-shift histogram. 


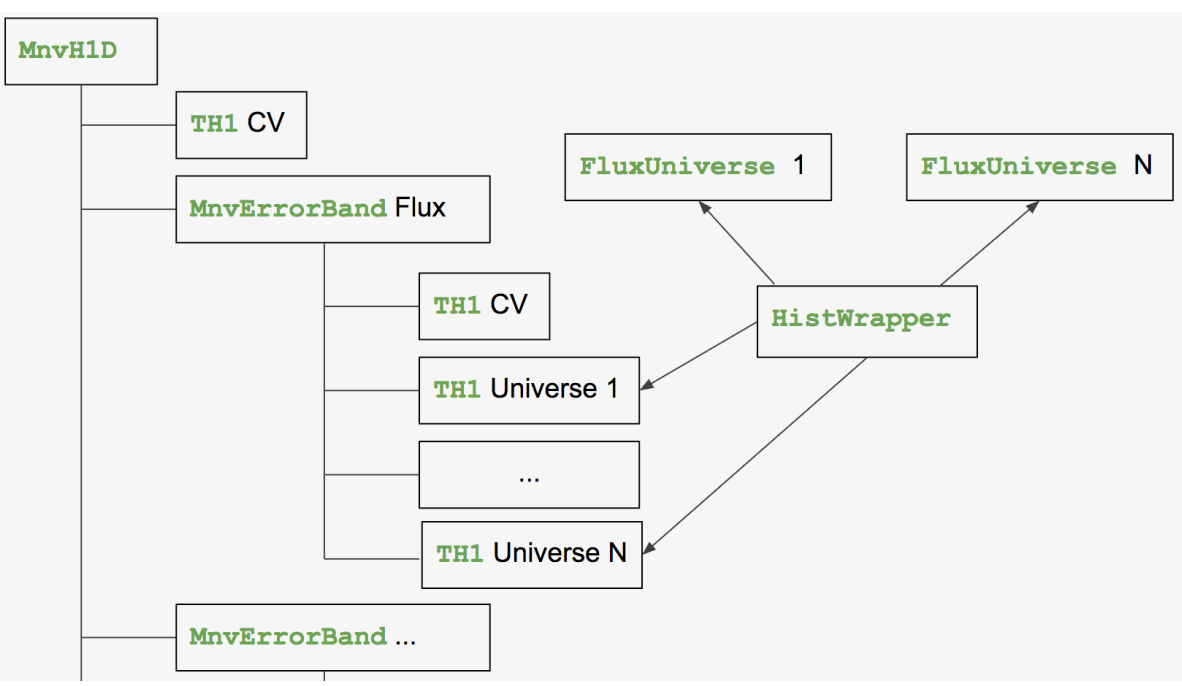

Figure C.2: Depiction of the HistWrapper and MnvH1D class structure. The MnvH1D class owns a simple TH1 histogram for the central value as well as an number of MnvErrorBand objects, which in turn own a copy of the central value TH1 and another TH1 for each systematically shifted distribution. A HistWrapper owns an MnvH1D as well as a map between the universe objects themselves and their corresponding TH1. In this depiction, the HistWrapper is mapping the Flux universe histograms to the universe objects.

Figure C.2 depicts the MnvH1D structure and how the HistWrapper object can map each universe histogram to the corresponding universe object that was used to initialize the object.

\section{C.4 TEMPLATE OUTPUT AND SAMPLE CODE}

C.3 shows the out-of-the box output of the new framework template code. Using a small data file, some basic cuts are applied and the neutrino energy is plotted. From the neutrino energy, the systematic uncertainty, broken down by categories can be calculated with the MnvH1D methods described in 7.1. Listings C.2, C.3, and C.4 show the main function code, the CVUniverse class, and an example user-defined systematic universe class. 


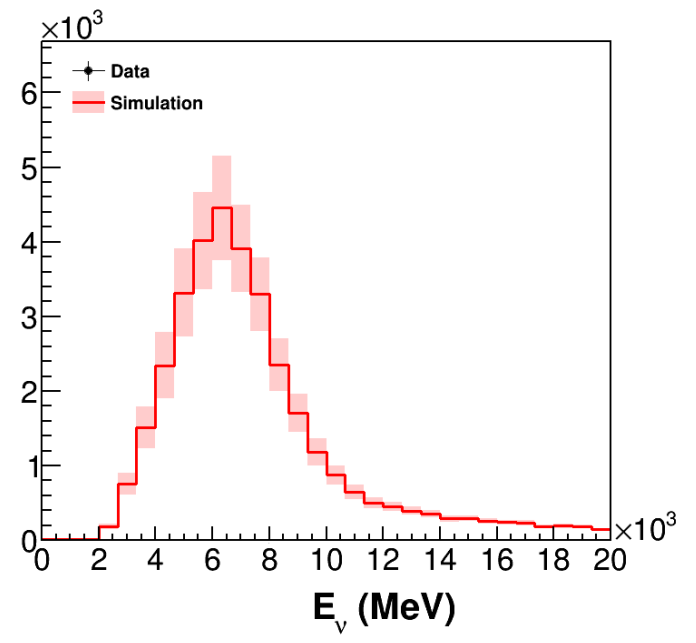

(a) Selected events in neutrino energy with systematic error band.

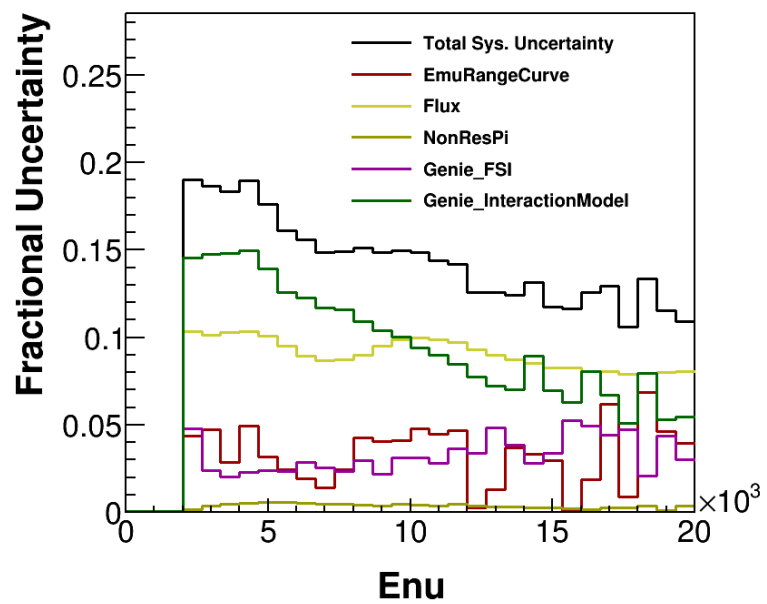

(b) Corresponding systematic error breakdown into error bands considered.

Figure C.3: Output of many-universe framework template code. 
Listing C.2: Framework template code - main script.

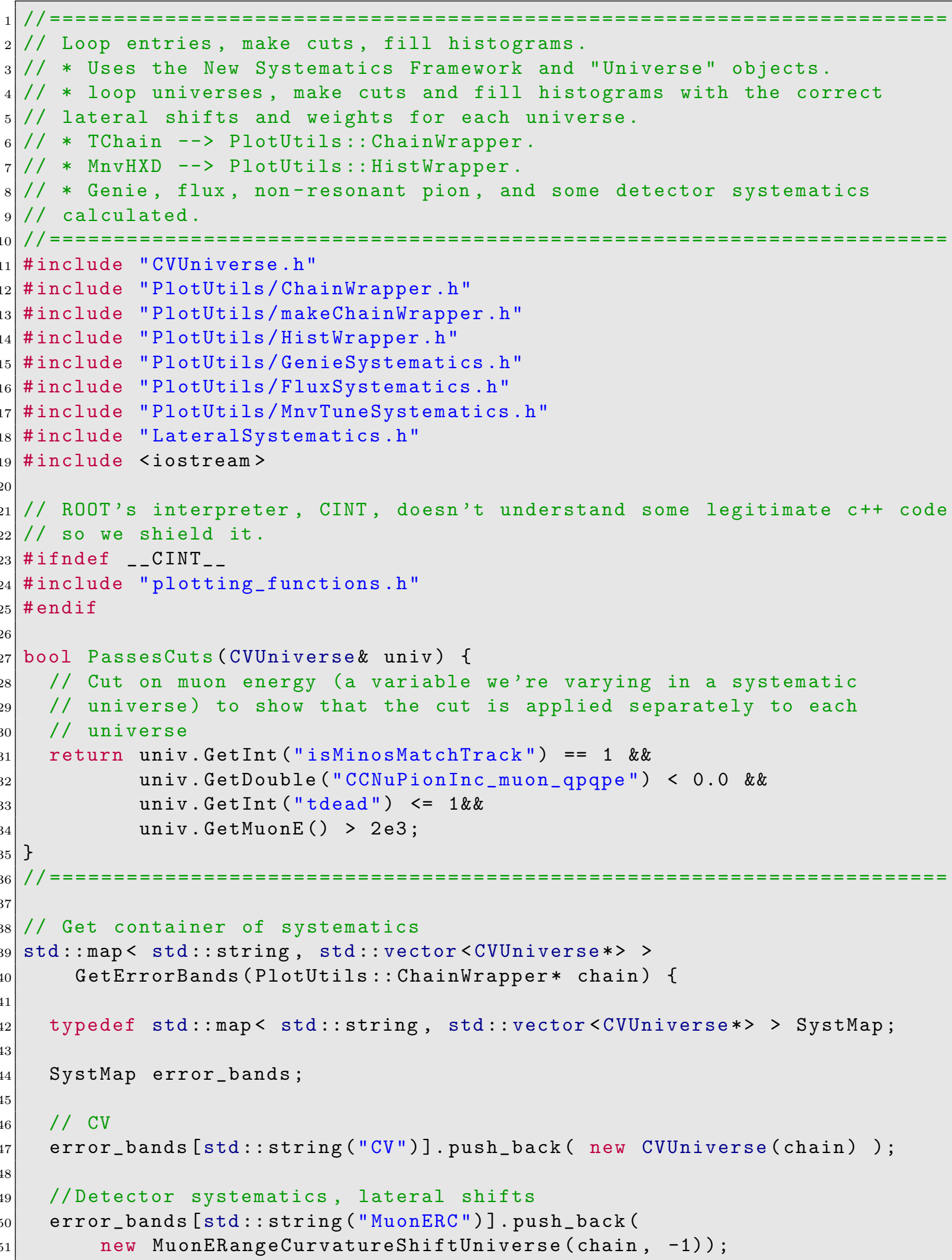




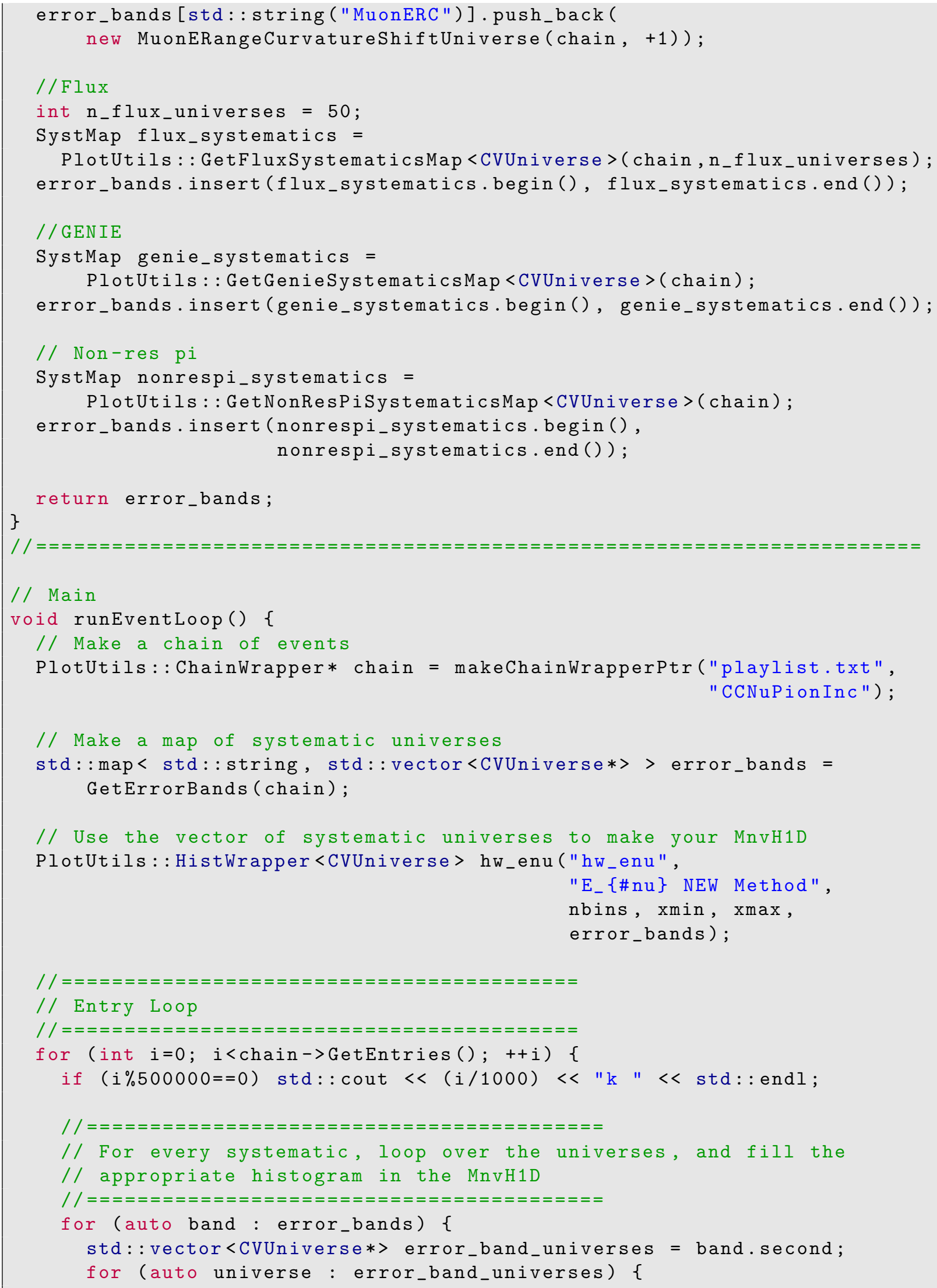




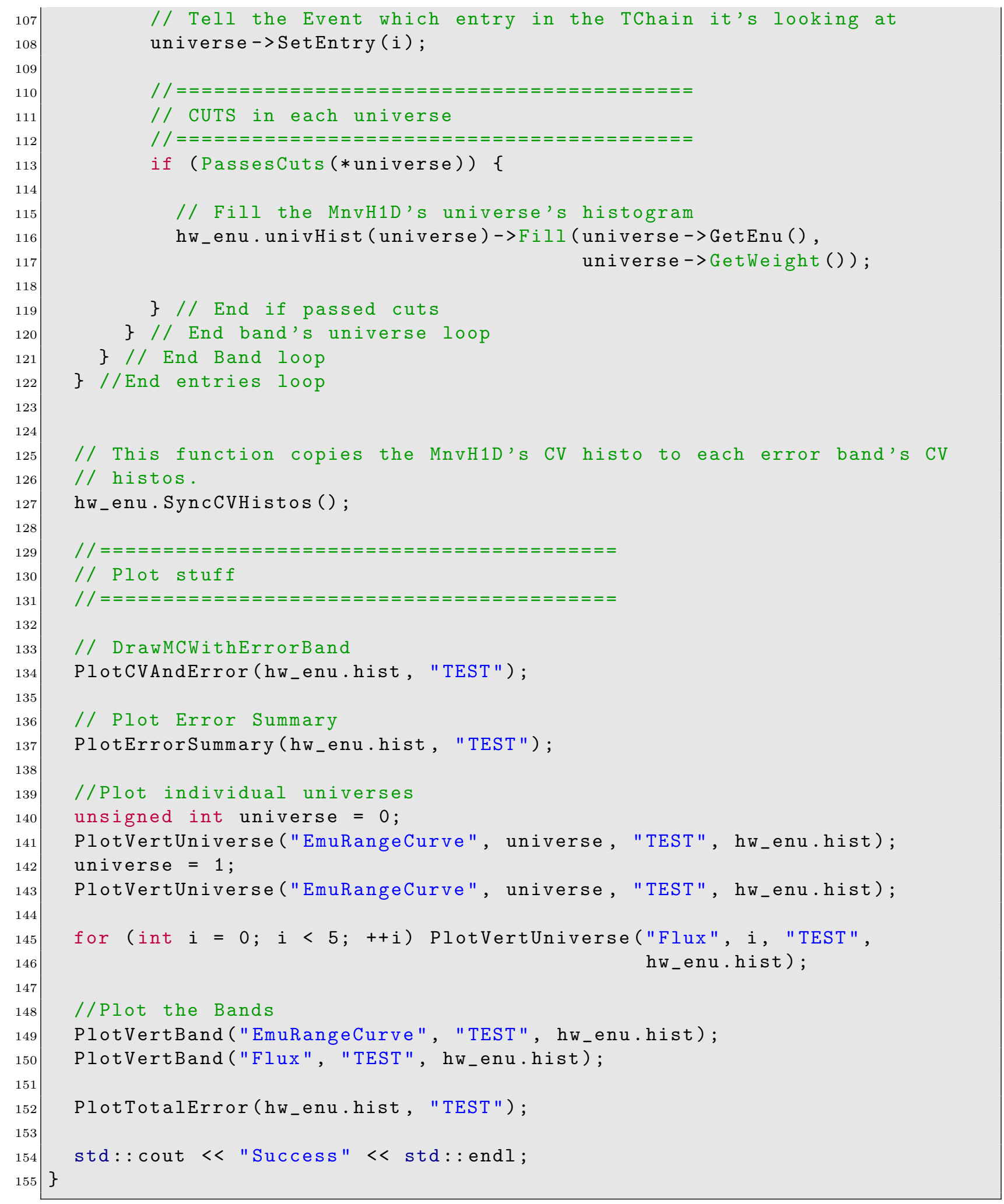


Listing C.3: Framework template code - user-defined CVUniverse class.

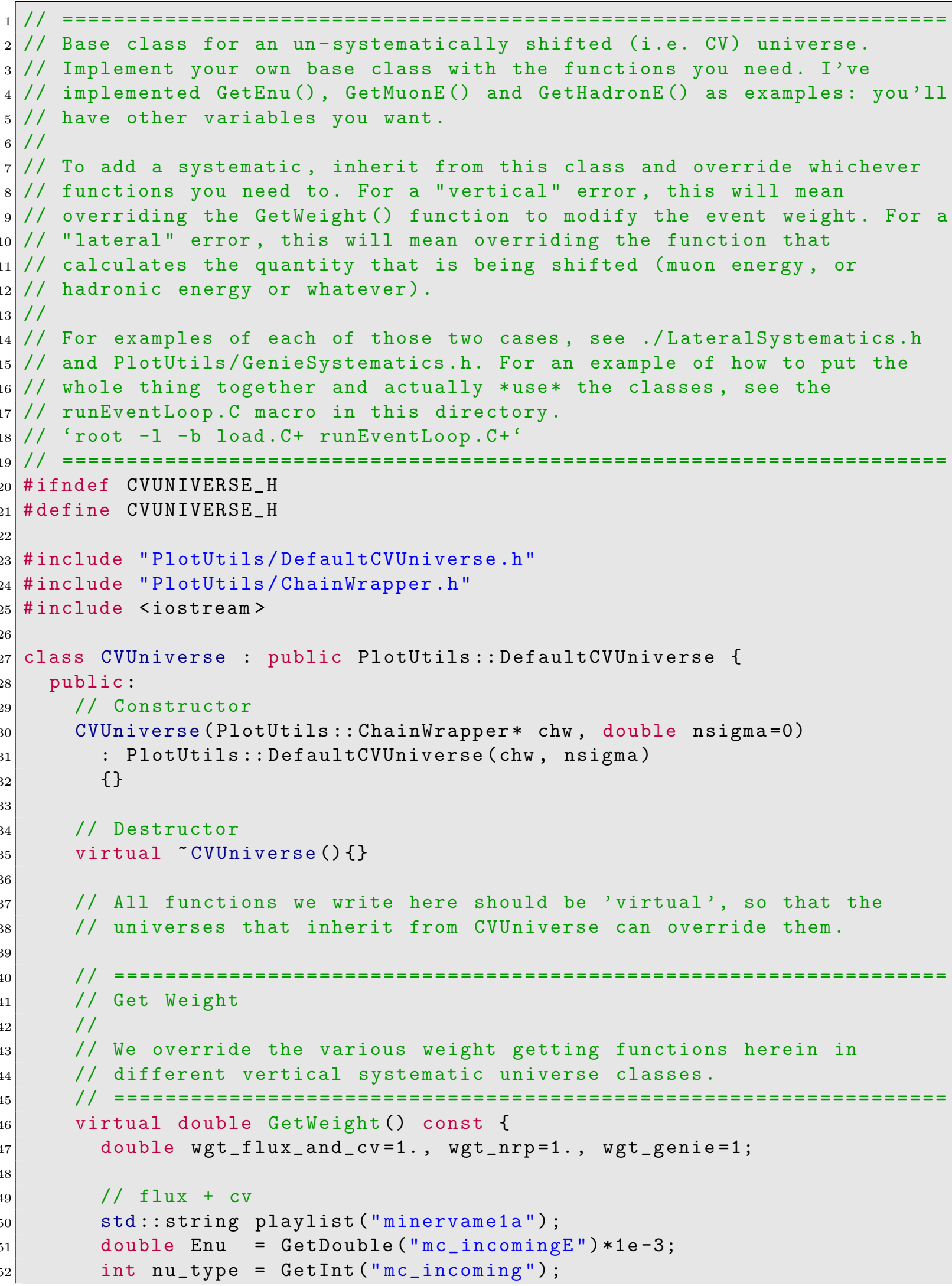




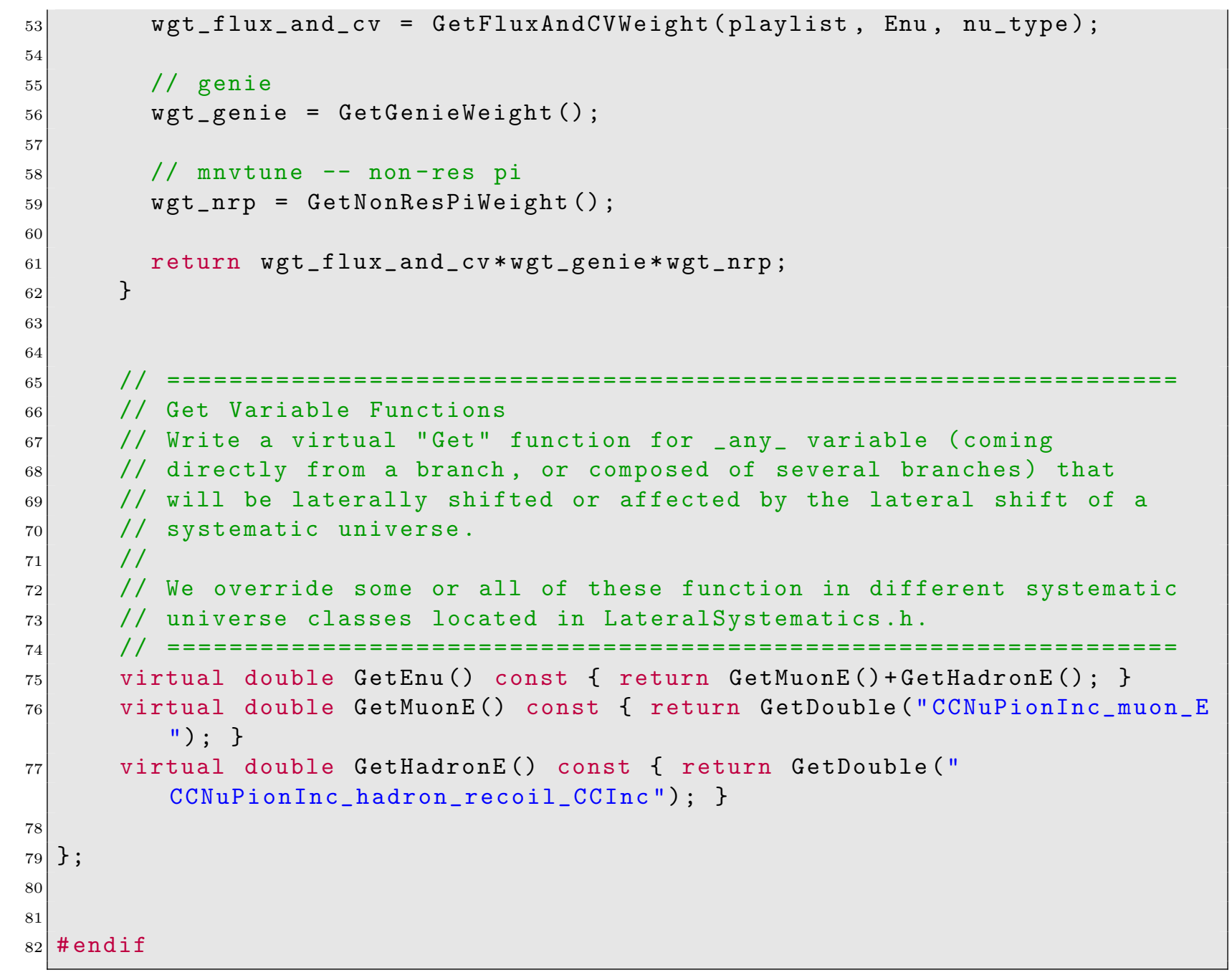


Listing C.4: Framework template code - user-defined systematic shifting the muon energy based on track range and curvature uncertainty.

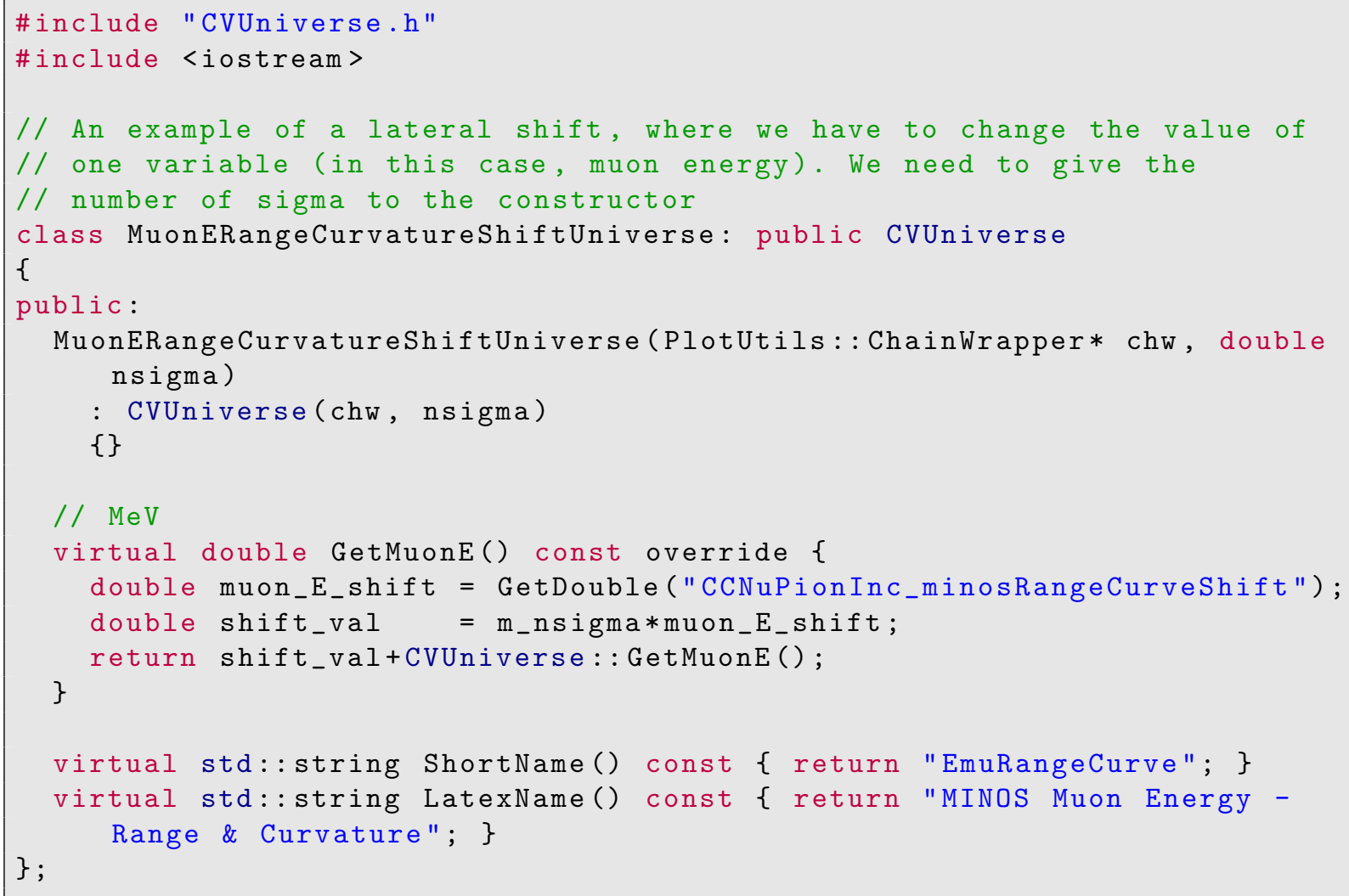




\section{APPENDIX D. THE MINERvA COLLABORATION}

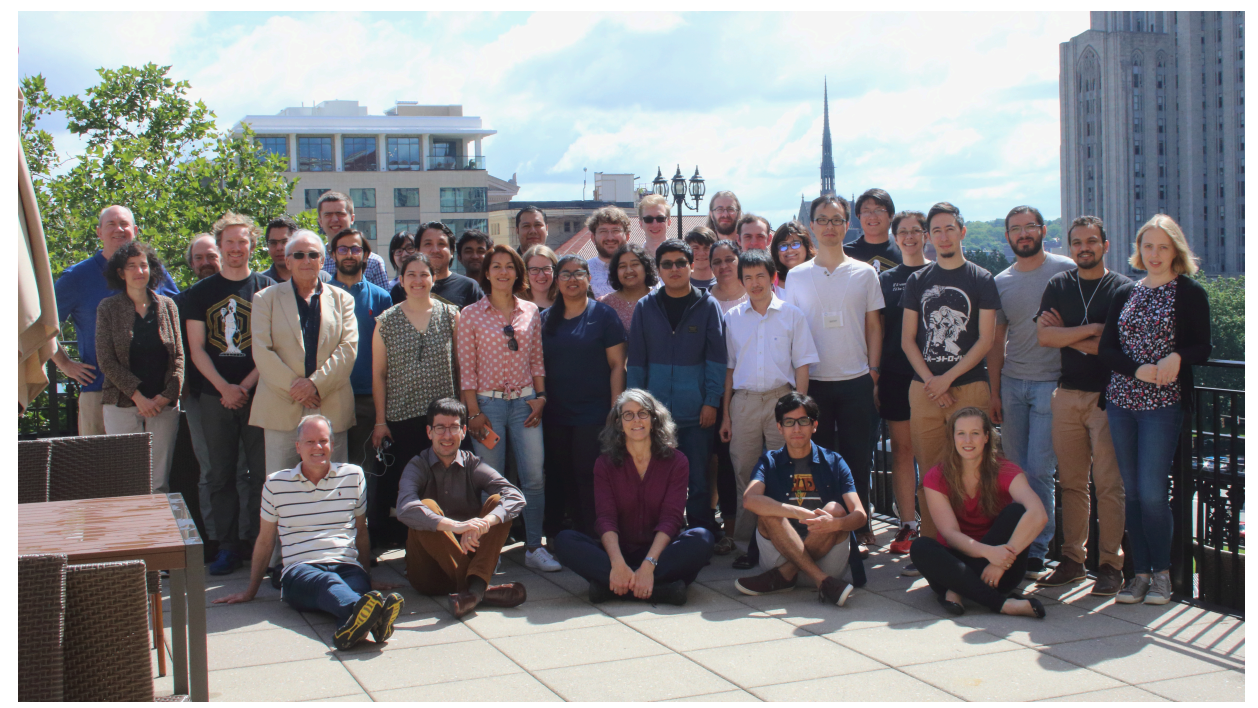

Figure D.1: MINERvA collaboration meeting — 2019 Pittsburgh, PA

Faiza Akbar, Mohammad Sajjad Athar, Zubair Ahmad Dar, Huma Haider

Aligarh Muslim University, Aligarh, India

Gian Caceres, Hlio da Motta, Adrian Lozano, Gilson Silva

Centro Brasileiro de Pesquisas Fsicas, Rio de Janeiro, Brazil

Omar Miranda, Luis Montano, Marco Torres

Centro de Investigacin y de Estudios Avanzados del Instituto Politcnico Nacional, Mexico City, Mexico

Jennica LeClerc, Christian Nguyen, Heather Ray 
University of Florida, Gainsville, Florida

Leo Bellantoni, Minerba Betancourt, Laura Fields, Deepika Jena, Jorge Morfin, Gabe Perdue Fermilab, Batavia, Illinois

Alessandro Bravar

Universit de Genve, Geneva, Switzerland

Diego Andrade, Jose Luis Bonilla, Julian Felix, Everardo Granados, Miguel Angel Hernandez Morquecho, Oscar E Moreno, Alejandro Ramirez Delgado, Francisco Rosas

Universidad de Guanajuato, Mexico

Emily Maher

Massachusetts College of Liberal Arts, North Adams, Massachusetts

Nishat Fiza, Satyajit Jena

Indian Institute of Science Education and Research, Mohali, India

Amit Bashyal, Mateus Carneiro, Sean Gilligan, Maggie Greenwood, Heidi Schellman, Noah Vaughan

Oregon State University, Corvallis, Oregon

Nathaniel Tagg

Otterbein College, Westerville, Ohio

David Coplowe, Xianguo Lu, David Wark, Alfons Weber, Kang Yang

University of Oxford, Oxford, United Kingdom

Steve Dytman, Ben Messerly, Donna Naples, Vittorio Paolone, Hang Su

University of Pittsburgh, Pittsburgh, Pennsylvania

Marvin Ascencio Sosa, Alberto Gago

Pontificia Universidad Catolica del Peru, Lima, Peru 
Aaron Bercellie, Arie Bodek, Howard Budd, Tejin Cai, Gonzalo Daz, Rob Fine, Sarah Henry, Ryan Howell, Jeffrey Kleykamp, Steve Manly, Kevin McFarland, Aaron McGowan, Andrew Olivier, Philip Rodrigues, Daniel Ruterbories, Mehreen Sultana, Clarence Wret University of Rochester, Rochester, New York

Nuruzzaman, Ronald Ransome

Rutgers University, New Brunswick, New Jersey

Hugh Gallagher, Trung Le, Tony Mann, Vladyslav Syrotenko Tufts University, Medford, Massachusetts

Kalven Bonin, Nafis Fuad, Rik Gran, Lauren Harewood

University of Minnesota-Duluth, Duluth, Minnesota

Ivancovish Julian Huancco Mamani, Carlos Solano Salinas

Universidad Nacional de Ingenieria, Lima, Peru

Jorge Chaves, David Last, Christopher Mauger

University of Pennsylvania, Philadelphia, Pennsylvania

William Brooks, Roger Galindo, Anushree Ghosh, Jonathan Miller, Barbara Yaeggy Universidad Tcnica Federico Santa Mara, Valparaso, Chile

Leonidas Aliaga Soplin, Amy Filkins, Cora Karamitsos, Michael Kordosky, Jeffrey Nelson, Edgar Valencia, Luis Zazueta

College of William and Mary, Williamsburg, Virginia

Jan Sobczyk

University of Wrocaw, Wrocaw, Poland

Deborah Harris

University of York, Toronto, Ontario, Canada 


\section{BIBLIOGRAPHY}

[1] Minerva technical design report. MINERvA Public Document 700-v28, 2006.

[2] K. Abe et al. Precise Measurement of the Neutrino Mixing Parameter $\theta_{23}$ from Muon Neutrino Disappearance in an Off-Axis Beam. Phys. Rev. Lett., 112(18):181801, 2014.

[3] K. Abe et al. First measurement of the muon neutrino charged current single pion production cross section on water with the T2K near detector. Phys. Rev., D95(1):012010, 2017.

[4] K. Abe et al. Search for $c p$ violation in neutrino and antineutrino oscillations by the t2k experiment with $2.2 \times 10^{21}$ protons on target. Phys. Rev. Lett., 121:171802, Oct 2018 .

[5] K. Abe et al. Measurement of the muon neutrino charged-current single $\pi^{+}$production on hydrocarbon using the T2K off-axis near detector ND280. 2019.

[6] R. Acciarri et al. Long-Baseline Neutrino Facility (LBNF) and Deep Underground Neutrino Experiment (DUNE), CDR Vol. 2. 2015.

[7] R. Acciarri et al. Long-Baseline Neutrino Facility (LBNF) and Deep Underground Neutrino Experiment (DUNE), CDR Vol. 1. 2016.

[8] R. Acciarri et al. First measurement of the cross section for $\nu_{\mu}$ and $\bar{\nu}_{\mu}$ induced single charged pion production on argon using ArgoNeuT. Phys. Rev., D98(5):052002, 2018.

[9] M. A. Acero et al. First Measurement of Neutrino Oscillation Parameters using Neutrinos and Antineutrinos by NOvA. Phys. Rev. Lett., 123(15):151803, 2019.

[10] C. Adams et al. The Intermediate Neutrino Program. In Workshop on the Intermediate Neutrino Program (WINP 2015) Upton, NY, USA, February 4-6, 2015, 2015.

[11] P. Adamson et al. Electron neutrino and antineutrino appearance in the full MINOS data sample. Phys. Rev. Lett., 110(17):171801, 2013.

[12] P. Adamson et al. Combined analysis of $\nu_{\mu}$ disappearance and $\nu_{\mu} \rightarrow \nu_{e}$ appearance in MINOS using accelerator and atmospheric neutrinos. Phys. Rev. Lett., 112:191801, 2014. 
[13] P. Adamson et al. The NuMI Neutrino Beam. Nucl. Instrum. Meth., A806:279-306, 2016.

[14] S. L. Adler. Tests of the conserved vector current and partially conserved axial-vector current hypotheses in high-energy neutrino reactions. Phys. Rev., 135:B963-B966, Aug 1964.

[15] S. Agostinelli et al. GEANT4: A Simulation toolkit. Nucl. Instrum. Meth., A506:250303, 2003.

[16] A. A. Aguilar-Arevalo et al. Measurement of the ratio of the $\nu_{\mu}$ charged-current singlepion production to quasielastic scattering with a 0.8 gev neutrino beam on mineral oil. Phys. Rev. Lett., 103:081801, Aug 2009.

[17] A. A. Aguilar-Arevalo et al. First Measurement of the Muon Neutrino Charged Current Quasielastic Double Differential Cross Section. Phys. Rev., D81:092005, 2010.

[18] A. A. Aguilar-Arevalo et al. Measurement of Neutrino-Induced Charged-Current Charged Pion Production Cross Sections on Mineral Oil at $\mathrm{E}_{\nu} \sim 1 \mathrm{GeV}$. Phys. Rev., D83:052007, 2011.

[19] L. Aliaga Soplin. Neutrino Flux Prediction for the NuMI Beamline. PhD thesis, WilliamMary Coll., 2016.

[20] L. Aliaga Soplin et al. Design, Calibration, and Performance of the MINERvA Detector. Nucl. Instrum. Meth., A743:130-159, 2014.

[21] L. Aliaga Soplin et al. Neutrino Flux Predictions for the NuMI Beam. Phys. Rev., D94(9):092005, 2016. [Addendum: Phys. Rev.D95,no.3,039903(2017)].

[22] L. Aliaga Soplin, L. Fields, T. Golan, J. Kiveni, and M. Kordosky. A brief documentation of the gen2 flux. Technical report, MINERvA, November 2015. MINERvA Technical Note No. TN004-v10.

[23] C. Alt et al. Inclusive production of charged pions in $\mathrm{p}+\mathrm{C}$ collisions at $158-\mathrm{GeV} / \mathrm{c}$ beam momentum. Eur. Phys. J., C49:897-917, 2007.

[24] O. Altinok et al. Measurement of $\nu_{\mu}$ charged-current single $\pi^{0}$ production on hydrocarbon in the few-GeV region using MINERvA. Phys. Rev., D96(7):072003, 2017.

[25] L. Alvarez-Ruso. Neutrino interactions: challenges in the current theoretical picture. Nucl. Phys. Proc. Suppl., 229-232:167-173, 2012.

[26] L. Alvarez-Ruso et al. NuSTEC White Paper: Status and challenges of neutrinonucleus scattering. Prog. Part. Nucl. Phys., 100:1-68, 2018. 
[27] C. Andreopoulos, C. Barry, S. Dytman, H. Gallagher, T. Golan, R. Hatcher, G. Perdue, and J. Yarba. The GENIE Neutrino Monte Carlo Generator: Physics and User Manual. 2015 .

[28] R. Barlow and C. Beeston. Fitting using finite monte carlo samples. Computer Physics Communications, 77(2):219 - 228, 1993.

[29] A. Bashyal. Flux fits for numi medium energy. MINERvA Internal Document 18780-v4, May 2018.

[30] O. Benhar, A. Fabrocini, S. Fantoni, and I. Sick. Spectral function of finite nuclei and scattering of GeV electrons. Nucl. Phys., A579:493-517, 1994.

[31] A. Bercellie. Pion production at minerva: Overview of the results from the low energy numi beam configuration. MINERvA, October 2018. NuInt 18 - 12th International Workshop on Neutrino-Nucleus Interactions in the Few-GeV Region.

[32] H. W. Bertini and M. P. Guthrie. News item results from medium-energy intranuclearcascade calculation. Nucl. Phys., A169:670-672, 1971.

[33] M. Betancourt et al. Comparisons and Challenges of Modern Neutrino Scattering Experiments (TENSIONS2016 Report). Phys. Rept., 773-774:1-28, 2018.

[34] J. B. Birks. Scintillations from Organic Crystals: Specific Fluorescence and Relative Response to Different Radiations. Proc. Phys. Soc., A64:874-877, 1951.

[35] A. Bodek, S. Avvakumov, R. Bradford, and Howard Scott Budd. Vector and Axial Nucleon Form Factors:A Duality Constrained Parameterization. Eur. Phys. J., C53:349$354,2008$.

[36] A Bodek, I Park, and U. Yang. Improved low q 2 model for neutrino and electron nucleon cross sections in few gev region. Nuclear Physics B-proceedings Supplements NUCL PHYS B-PROC SUPPL, 139:113-118, 022005.

[37] A. Bodek and J. L. Ritchie. Fermi Motion Effects in Deep Inelastic Lepton Scattering from Nuclear Targets. Phys. Rev., D23:1070, 1981.

[38] A Bodek and U. K. Yang. Higher twist, xi(omega) scaling, and effective LO PDFs for lepton scattering in the few GeV region. J. Phys., G29:1899-1906, 2003.

[39] T Cai, X Lu, and D Ruterbories. Pion-proton correlation in neutrino interactions on nuclei. Phys. Rev., D100:073010, 2019.

[40] The Dune Collaboration. Dune: Deep underground neutrino experiment, an international experiment for neutrino science. https://www.dunescience.org/, Accessed: 2019-11-05. 
[41] The MINERvA Collaboration. Updated charged pion production results. https:// minerva.fnal.gov/wp-content/uploads/2017/03/Updated_1pi_data.pdf, Accessed: 2019-11-1.

[42] M. Derrick et al. Study of single-pion production by weak neutral currents in low-energy

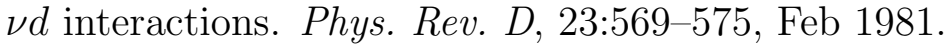

[43] E. C. Dukes, R. Ehrlich, S. Goadhouse, L. Mualem, A. Norman, and R. Tesarek. The NOvA power distribution system. Nucl. Instrum. Meth., A902:123-137, 2018.

[44] B. Eberly et al. Charged pion production in $\nu_{\mu}$ interactions on hydrocarbon at $\left\langle E_{\nu}\right\rangle=$ 4.0 GeV. Phys. Rev., D92(9):092008, 2015.

[45] B M. Eberly. Characterization of Final State Interaction Strength in Plastic Scintillator by Muon-Neutrino Charged Current Charged Pion Production. PhD thesis, Pittsburgh U., 2014.

[46] K. S. Egiyan et al. Measurement of 2- and 3-nucleon short range correlation probabilities in nuclei. Phys. Rev. Lett., 96:082501, 2006.

[47] Fermilab. Fermilab target systems. https://targets.fnal.gov/NuMI_target.html, Accessed: 2019-10-10.

[48] A Ferrari, P Sala, A Fasso, and J. Ranft. Fluka: a multi-particle transport code. CERN Yellow report, 2005-10, 012005.

[49] R. P. Feynman, M. Kislinger, and F. Ravndal. Current matrix elements from a relativistic quark model. Phys. Rev., D3:2706-2732, 1971.

[50] L. Fields. Muon energy scale investigations. MINERvA Internal Document 19423-v1, July 2018.

[51] R. Fine. Chronobuncher update. MINERvA Internal Document 15047-v1, July 2017.

[52] R. Fine. Minerva: Past, present, and future. Fermilab, June 2019. 52nd Annual Fermilab Users Meeting.

[53] J. A. Formaggio and G. P. Zeller. From eV to EeV: Neutrino Cross Sections Across Energy Scales. Rev. Mod. Phys., 84:1307-1341, 2012.

[54] F Foster and G Hughes. Electroproduction of nucleon resonances. Reports on Progress in Physics, 46(12):1445-1489, dec 1983.

[55] A. Ghosh. Minos matched muons tracking efficiency at medium energy: End game. MINERvA Internal Document 20760-v1, October 2018.

[56] A. Gil, J. Nieves, and E. Oset. Many body approach to the inclusive (e, e-prime) reaction from the quasielastic to the Delta excitation region. Nucl. Phys., A627:543-598, 1997. 
[57] C Giunti and C W. Kim. Fundamentals of Neutrino Physics and Astrophysics. 2007.

[58] R. Gran. Birks law parameter from minerva testbeam data. Technical report, Fermilab, November 2014. MINERvA Technical Note No. 037.

[59] R. Gran. Model Uncertainties for Valencia RPA Effect for MINERvA. 2017.

[60] R. Gran, J. Nieves, F. Sanchez, and M. J. Vicente Vacas. Neutrino-nucleus quasi-elastic and 2p2h interactions up to $10 \mathrm{GeV}$. Phys. Rev., D88(11):113007, 2013.

[61] D. J. Griffiths. Introduction to elementary particles; 2nd rev. version. Physics textbook. Wiley, New York, NY, 2008.

[62] M. P. Guthrie, R. G. Alsmiller, and H. W. Bertini. Calculation of the capture of negative pions in light elements and comparison with experiments pertaining to cancer radiotherapy. Nucl. Instrum. Meth., 66:29-36, 1968.

[63] R. Hatcher. Proposal for a unified flux n-tuple format. MINOS Internal Document 9070-v4, October 2012.

[64] A. Higuera et al. Measurement of Coherent Production of $\pi^{ \pm}$in Neutrino and Antineutrino Beams on Carbon from $E_{\nu}$ of 1.5 to $20 \mathrm{GeV}$. Phys. Rev. Lett., 113(26):261802, 2014.

[65] A. Holin. Beam fit for minos+ analysis. NuMI-X Internal Document 88-v1, October 2014.

[66] J. Hylen. Target location along beam-line; summary of entire minos run. MINOS Internal Document 9314-v1, July 2012.

[67] J. Hylen. Numi horn 1 mis-alignment. NuMI-X Internal Document 131-v1, November 2016.

[68] M Kabirnezhad. Single pion production in neutrino-nucleon Interactions. Phys. Rev., D97(1):013002, 2018.

[69] A. B. Kaidalov. The Quark-Gluon Structure of the Pomeron and the Rise of Inclusive Spectra at High-Energies. Phys. Lett., 116B:459-463, 1982.

[70] T Katori and M Martini. Neutrinonucleus cross sections for oscillation experiments. $J$. Phys., G45(1):013001, 2018.

[71] T. Kitagaki et al. Charged-current exclusive pion production in neutrino-deuterium interactions. Phys. Rev. D, 34:2554-2565, Nov 1986.

[72] Z. Koba, Holger Bech Nielsen, and P. Olesen. Scaling of multiplicity distributions in high-energy hadron collisions. Nucl. Phys., B40:317-334, 1972. 
[73] S. Kopp, Z. Pavlovic, and D. Indurthy. Systematic uncertainties in the numi beam flux. MINOS Internal Document 1283-v3, May 2007.

[74] K. S. Kuzmin, V. V. Lyubushkin, and V. A. Naumov. Axial masses in quasielastic neutrino scattering and single-pion neutrinoproduction on nucleons and nuclei. Acta Phys. Polon., B37:2337-2348, 2006.

[75] K. S. Kuzmin, V. V. Lyubushkin, and V. A. Naumov. Fine-tuning parameters to describe the total charged-current neutrino-nucleon cross section. Phys. Atom. Nucl., 69:1857-1871, 2006 .

[76] K. S. Kuzmin, V. V. Lyubushkin, and V. A. Naumov. Quasielastic axial-vector mass from experiments on neutrino-nucleus scattering. Eur. Phys. J., C54:517-538, 2008.

[77] T. Le et al. Single Neutral Pion Production by Charged-Current $\bar{\nu}_{\mu}$ Interactions on Hydrocarbon at $\left\langle E_{\nu}\right\rangle=3.6 \mathrm{GeV}$. Phys. Lett., B749:130-136, 2015.

[78] P. Lebrun. On systematic uncertainty on the neutrino flux due to geant4 geometry modeling, specifically, horn1. Technical report, Fermilab, December 2014. MINERvA Internal Document 10573-v1.

[79] P. Lebrun. On focusing uncertainties of the numi beam. Technical report, Fermilab, November 2017. MINERvA Internal Document 23542-v1.

[80] C. H. Llewellyn Smith. Neutrino Reactions at Accelerator Energies. Phys. Rept., 3:261$379,1972$.

[81] K Mahn, C Marshall, and C Wilkinson. Progress in Measurements of 0.110 GeV NeutrinoNucleus Scattering and Anticipated Results from Future Experiments. Ann. Rev. Nucl. Part. Sci., 68:105-129, 2018.

[82] C. Marshall. Measurement of charged kaon production by neutrinos at MINERvA. PhD thesis, U. Rochester, 2016.

[83] M. Martini, M. Ericson, G. Chanfray, and J. Marteau. A Unified approach for nucleon knock-out, coherent and incoherent pion production in neutrino interactions with nuclei. Phys. Rev., C80:065501, 2009.

[84] C. L. McGivern et al. Cross sections for $\nu_{\mu}$ and $\bar{\nu}_{\mu}$ induced pion production on hydrocarbon in the few-GeV region using MINERvA. Phys. Rev., D94(5):052005, 2016.

[85] B. Messerly. Flux focusing ratios - daisy binning. MINERvA Internal Document 14064v3, May 2017.

[86] B. Messerly. Me flux focusing uncertainties update. MINERvA Internal Document 12955-v2, February 2017. 
[87] B. Messerly. Me flux uncertainties update. MINERvA Internal Document 14396-v3, June 2017.

[88] B. Messerly. Me focusing uncertainties update. MINERvA Internal Document 12965-v1, September 2017.

[89] B. Messerly, J. Ellison, D. Friant, A. Lister, and S. Mandalia. Neutrino detector livingston plots. Fermilab, August 2017. 10th International Neutrino Summer School 2017.

[90] D. G. Michael et al. The Magnetized steel and scintillator calorimeters of the MINOS experiment. Nucl. Instrum. Meth., A596:190-228, 2008.

[91] R. Milburn. Neutrino beam simulation using paw with weighted monte carlo. MINOS Internal Document 109-v1, October 1995.

[92] Hisakazu Minakata. An Effective Two-Flavor Approximation for Neutrino Survival Probabilities in Matter. JHEP, 05:043, 2017.

[93] MissMJ. Particle content of the standard model. https://commons.wikimedia.org/ wiki/File:Standard_Model_of_Elementary_Particles.svg, Accessed: 2019-11-1.

[94] U. Mosel and K. Gallmeister. Muon-neutrino-induced charged current pion production on nuclei. Phys. Rev., C96(1):015503, 2017. [Addendum: Phys. Rev.C99,no.3,035502(2019)].

[95] J. Neyman and E. S. Pearson. On the problem of the most efficient tests of statistical hypotheses. Philosophical Transactions of the Royal Society of London. Series A, Containing Papers of a Mathematical or Physical Character, 231:289-337, 1933.

[96] J. Nieves, Jose Enrique Amaro, and M. Valverde. Inclusive quasi-elastic neutrino reactions. Phys. Rev., C70:055503, 2004. [Erratum: Phys. Rev.C72,019902(2005)].

[97] J Nieves, I R Simo, F Snchez, and M J. Vicente Vacas. 2p2h Excitations, MEC, Nucleon Correlations and Other Sources of QE-like Events. JPS Conf. Proc., 12:010002, 2016.

[98] J. Nieves, I. Ruiz Simo, and M. J. Vicente Vacas. Inclusive charged-current neutrinonucleus reactions. Phys. Rev. C, 83:045501, Apr 2011.

[99] B Nilsson-Almqvist and E Stenlund. Interactions Between Hadrons and Nuclei: The Lund Monte Carlo, Fritiof Version 1.6. Comput. Phys. Commun., 43:387, 1987.

[100] J. M. Paley et al. Measurement of Charged Pion Production Yields off the NuMI Target. Phys. Rev., D90(3):032001, 2014.

[101] X. Qian and P. Vogel. Neutrino Mass Hierarchy. Prog. Part. Nucl. Phys., 83:1-30, 2015. 
[102] G. M. Radecky et al. Study of Single Pion Production by Weak Charged Currents in Low-energy Neutrino d Interactions. Phys. Rev., D25:1161-1173, 1982. [Erratum: Phys. Rev.D26,3297(1982)].

[103] J Rademacker. An exact formula to describe the amplification process in a photomultiplier tube. Nuclear Instruments and Methods in Physics Research Section A: Accelerators, Spectrometers, Detectors and Associated Equipment, 484(1):432 - 443, 2002.

[104] D Rein and L M. Sehgal. Neutrino Excitation of Baryon Resonances and Single Pion Production. Annals Phys., 133:79-153, 1981.

[105] D Rein and L M. Sehgal. Coherent pi0 Production in Neutrino Reactions. Nucl. Phys., B223:29-44, 1983.

[106] D. Rein and L. M. Sehgal. PCAC and the Deficit of Forward Muons in pi+ Production by Neutrinos. Phys. Lett., B657:207-209, 2007.

[107] L. Ren et al. Measurement of the antineutrino to neutrino charged-current interaction cross section ratio in MINERvA. Phys. Rev., D95(7):072009, 2017. [Addendum: Phys. Rev.D97,no.1,019902(2018)].

[108] D. Rimal. Inclusive antineutrino events in fhc. MINERvA Internal Document 12636-v1, October 2016.

[109] D. Rimal. Zero horn current data (in me era). MINERvA Internal Document 12871-v1, February 2017.

[110] P. Rodrigues. Me beam position. MINERvA Internal Document 12895-v1, February 2017.

[111] P. Rodrigues et al. Identification of nuclear effects in neutrino-carbon interactions at low three-momentum transfer. Phys. Rev. Lett., 116:071802, 2016. [Addendum: Phys. Rev. Lett.121,no.20,209902(2018)].

[112] P. Rodrigues, C. Wilkinson, and K. McFarland. Constraining the GENIE model of neutrino-induced single pion production using reanalyzed bubble chamber data. Eur. Phys. J., C76(8):474, 2016.

[113] A. Rodriguez et al. Measurement of single charged pion production in the chargedcurrent interactions of neutrinos in a 1.3-GeV wide band beam. Phys. Rev., D78:032003, 2008.

[114] S. Ross. Peirce's criterion for the elimination of suspect experimental data. J. Eng. Technol., 20, 092003.

[115] T Sjostrand, S Mrenna, and P Z. Skands. PYTHIA 6.4 Physics and Manual. JHEP, 05:026, 2006. 
[116] R. A. Smith and E. J. Moniz. NEUTRINO REACTIONS ON NUCLEAR TARGETS. Nucl. Phys., B43:605, 1972. [Erratum: Nucl. Phys.B101,547(1975)].

[117] J T. Sobczyk and J Żmuda. Investigation of recent weak single-pion production data. Phys. Rev. C, 91:045501, Apr 2015.

[118] P. Stowell et al. Tuning the GENIE Pion Production Model with MINERvA Data. 2019.

[119] R. Subedi et al. Probing Cold Dense Nuclear Matter. Science, 320:1476-1478, 2008.

[120] N. Tagg et al. Arachne - A web-based event viewer for MINERvA. Nucl. Instrum. Meth., A676:44-49, 2012.

[121] M. et. al. Tanabashi. Review of particle physics. Phys. Rev. D, 98:030001, Aug 2018.

[122] E. A. Uehling and G. E. Uhlenbeck. Transport phenomena in einstein-bose and fermidirac gases. i. Phys. Rev., 43:552-561, Apr 1933.

[123] E. Valencia et al. Constraint of the MINERvA Medium Energy Neutrino Flux using Neutrino-Electron Elastic Scattering. 2019.

[124] J. W. Van Orden and T. W. Donnelly. Mesonic processes in deep inelastic electron scattering from nuclei. Annals Phys., 131:451-493, 1981.

[125] C. Wilkinson. Overview of neutrino-nucleus interaction physics. T2K Collaboration, October 2017. 17th International Workshop on Next Generation Nucleon Decay and Neutrino Detectors.

[126] C Wilkinson, P Rodrigues, S Cartwright, L Thompson, and K McFarland. Reanalysis of bubble chamber measurements of muon-neutrino induced single pion production. Phys. Rev., D90(11):112017, 2014.

[127] J. Wolcott. Impact of cross section uncertainties on nova oscillation analyses. NOvA Collaboration, August 2018. NuFact 2018, 20th workshop on neutrinos from accelerators.

[128] J. Wolcott. New neutrino and antineutrino oscillation results from nova. NOvA Collaboration, June 2019. 52nd Annual Fermilab Users Meeting.

[129] T. Yang, C. Andreopoulos, H. Gallagher, K. Hoffmann, and P. Kehayias. A Hadronization Model for Few-GeV Neutrino Interactions. Eur. Phys. J., C63:1-10, 2009. 